DEPARTAMENTO DE MATEMÁTICA APLICADA

Instituto Universitario de Matemática Pura y Aplicada

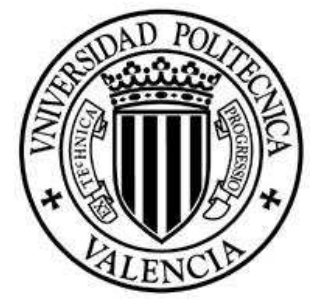

\title{
Compacta in Banach spaces
}

\section{PhD THESIS}

Author: Alma Lucía González Correa

Supervised by: Dr. Vicente Montesinos Santalucía

Dr. Marián Fabian

September 2008 

Vicente Montesinos Santalucía, Full Professor of the Departamento de Matemática Aplicada at the Universidad Politécnica de Valencia and Marián Fabian, Head of Research at the Institute of Mathematics of the Czech Academy of Sciences

CERTIFIE that this Memoir: "Compacta in Banach spaces", has been realized under our supervision by Mrs. Alma Lucía González Correa in order to get the PhD degree in Mathematics.

For the record and law enforcement in effect, we sign this certificate in Valencia, September the 16th., 2008. 




\section{Acknowledgements}

I thank my supervisors, Professor Marián Fabian, from the Institute of Mathematics of the Czech Academy of Sciences, and Professor Vicente Montesinos, from the Instituto de Matemática Pura y Aplicada of the Universidad Politécnica de Valencia, for their continuous help, encouragement, and support provided along these years. I also have learned of their discipline and their human qualities. Their advice and suggestions have been invaluable.

It is almost impossible to provide a complete list of the many mathematicians that contributed somehow to this work. Special thanks are due to Professor Václav Zizler, from the Institute of Mathematics of the Czech Academy of Sciences, for his many contributions to different topics of this Memoir, especially Chapter 5. He provided several important seminal ideas around the subject of flatness and generation. Among others, let me mention M. Valdivia, J. Orihuela, B. Cascales, P. Hájek and O. Kalenda. I thank specially the referees who wrote their reports, M. Valdivia, V. Zizler and J. Orihuela.

I want also thank the Instituto de Matemática Pura y Aplicada of the Universidad Politécnica de Valencia for the hospitality offered and facilities provided. In particular, thanks are due to his Director, Professor José Bonet. Along these years, I enjoyed the friendship and support of many people at the Instituto (José, Enrique, David, Lluis, Olvido,...). A special mention deserve my two room-mates Irene and Jordi. They provided everything to make me life pleasant.

Everything was made possible by the financial support of the Mexican Government by a grant from Consejo Nacional de Ciencia y Tecnología and of the Spanish Goverment by a grant from Fundación Carolina, that allowed me to stay in Spain. The officers who were responsible of it have been always very receptive and supportive. Another source of financial support has been the Proyecto MTM2005-08210 of the Spanish Ministerio de Educación y Ciencia and its head of research Professor Manuel Maestre. I want also to thank the Institute of Mathematics of the Czech Academy of Sciences for its hospitality, and in particular, the financial support of Grants AVOZ 10190503 and IAA 100190610.

Let me go now to more personal considerations. My thoughts go also to my family. From the distance, they encouraged my work explicitly and, I am sure, they prayed for me. Last, and certainly not least, I want to thank my husband for his love, patience, understanding and moral support. He believe in me and this is something I will never forget. 



\section{Resumen}

Capítulo 1. Después de estudiar algunos preliminares sobre familias adecuadas de conjuntos, formulamos y probamos algunas equivalencias, cada una de ellas son una condición suficiente para que la familia defina un conjunto compacto de Gul'ko. Damos una caracterización de conjunto compacto de Gul'ko en términos de emparejamiento con un conjunto $\mathcal{K}$-analítico.

Capítulo 2. Estudiamos propiedades de los espacios de Banach débilmente Lindelöf determinados no-separables. Damos una caracterización por medio de la existencia de un generador proyeccional full sobre él. Estudiamos algunos aspectos sobre sistemas biortogonales en espacios de Banach. Usando técnicas de resoluciones proyeccionales de la identidad, probamos una extensión de un resultado de Argyros y Mercourakis.

Capítulo 3. En el espacio $\left(c_{0}(\Gamma),\|\cdot\|_{\infty}\right)$, con $\Gamma \in \mathbb{R}$, damos una norma equivalente estrictamente convexa.

Capítulo 4. Consideramos una caracterización de los subespacios de espacios de Banach débilmente compactamente generados, en términos de una propiedad de cubrimiento de la bola unidad por medio de conjuntos $\epsilon$-débilmente compactos. Reemplazamos este concepto por otro más preciso que llamamos $\epsilon$-débilmente auto-compactos, este concepto permite una mejor descripción.

Capítulo 5. Damos condiciones intrínsecas, necesarias y suficientes para que un espacio de Banach sea generado por $c_{0}(\Gamma)$ o $\ell_{p}(\Gamma)$ para $p \in(1,+\infty)$. Ofrecemos una nueva demostración de un resultado de Rosenthal, sobre operadores de $c_{0}(\Gamma)$ en un espacio de Banach. 



\section{Resum}

Capítol 1. Imposem condicions a famílies adequades de conjunts per tal de donar condicions equivalents que impliquen que la família de conjunts definisca un compacte de Gul'ko. Caracteritzem els compactes de Gul'ko en termes de paritat.

Capítol 2. Tracta de la classe d'espais de Banach no separables que són dèbil Lindelöf determinats. Caracteritzem aquests espais en termes de l'existència d'un generador projectiu. Estudiem algunes qüestions sobre sistemes biortogonals en espais de Banach. Utilitzant resolucions projectives de la identitat, estenem un resultat de Argyros i Mercourakis.

Capítol 3. En el espai $\left(c_{0}(\Gamma),\|\cdot\|_{\infty}\right), \Gamma \in \mathbb{R}$, donem una norma equivalent que és estrictament convexa.

Capítol 4. Considerem una caracterització de subespais en la classe d'espais de Banach dèbil compactament generats mitjançant una propietat de cobriment de la bola unitat tancada amb conjunts $\epsilon$-débil compactes. Modifiquem aquest concepte amb altre que anomenem $\epsilon$-dèbil autocompacte. L' $\epsilon$-dèbil autocompacitat ens permet donar una millor descripció.

Capítol 5. Donem condicions necessàries i suficients intrínseques perquè un espai de Banach $X$ siga generat per $c_{0}(\Gamma)$ o $\ell_{p}(\Gamma), p \in(1,+\infty)$. Finalment, donem una nova demostració d'un resultat de Rosenthal sobre operadors de $c_{0}(\Gamma)$ a un espai de Banach. 



\section{Abstract}

Chapter 1. After some preliminaries dealing with adequate families of sets, we formulate and prove some equivalences, all of them implying that the family defines a Gul'ko compactum. We provide a characterization of Gul'ko compacta in terms of pairings.

Chapter 2. Deals with the class of non-separable weakly Lindelöf determined Banach spaces and their relatives. We give a characterization of weakly Lindelöf determined Banach spaces by mean of the existence of a full projectional generator on it. We study some remarks on biorthogonal systems in Banach spaces. We prove by the technology of PRI's, an extension of a result due to Argyros and Mercourakis.

Chapter 3. For $\left(c_{0}(\Gamma),\|\cdot\|_{\infty}\right)$, with $\Gamma \subset \mathbb{R}$, we provide an equivalent norm on $c_{0}(\Gamma)$ that is strictly convex.

Chapter 4. We consider a characterization of subspaces of the class of weakly compactly generated Banach spaces in terms of a covering property of the closed unit ball, by means of $\epsilon$-weakly compact sets. We replace this concept by a more precise one that we call $\epsilon$-weakly self-compactness, this concept allows a better description.

Chapter 5. We give intrinsic necessary and sufficient conditions for a Banach space $X$ to be generated by $c_{0}(\Gamma)$ or $\ell_{p}(\Gamma)$ for $p \in(1,+\infty)$. As a byproduct we give a new proof of a result of Rosenthal on operators from $c_{0}(\Gamma)$ into Banach spaces. 



\section{Contents}

Introduction, Summary and Notation 1

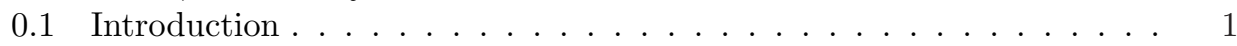

0.2 Notation . . . . . . . . . . . . . . . . . . . . 2

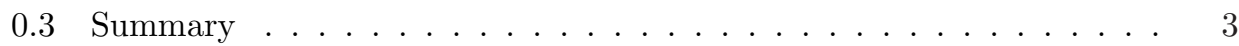

0.4 A matter of contrast . . . . . . . . . . . . . . . . 9

1 Gul'ko compacta 11

2 WCG Banach spaces and their relatives 21

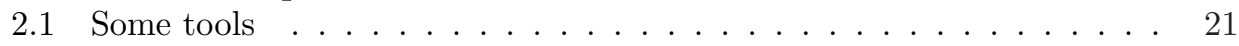

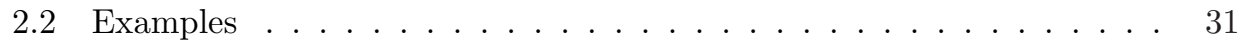

2.2.1 WCG Banach spaces . . . . . . . . . . . . . . . . 31

2.2.2 WCD Banach spaces . . . . . . . . . . . . . . . 33

2.2.3 WLD Banach spaces . . . . . . . . . . . . . . . 35

2.3 Biorthogonal systems in WCG Banach

spaces .......................... 40

$\begin{array}{lll}3 & \text { A renorming result } & 49\end{array}$

4 Some remarks on Krein's theorem $\quad 53$

4.1 Upper envelopes and distances . . . . . . . . . . . . . . . . . 53

4.2 Quantitative Krein's Theorem . . . . . . . . . . . . . . . . . 56

4.2.1 Interchanging limits and $\varepsilon$-WSK sets . . . . . . . . . . . . . 56

4.2.2 $\varepsilon$-WSK sets in natural settings . . . . . . . . . . . . . 61

$5 \quad$ Flat sets, $\ell_{p}$-generating and fixing $c_{0}$ in nonseparable setting $\quad 63$

5.1 Introduction . . . . . . . . . . . . . . . . . . . . . . . . . . 63

5.2 Asymptotically $p$-flat and $p$-flat sets . . . . . . . . . . . . . . . 65

5.2.1 Asymptotically $p$-flat sets . . . . . . . . . . . . . 65

5.2.1.1 Basic results and some remarks . . . . . . . . . 65

5.2 .1 .2 Some stability properties . . . . . . . . . . 76

5.2.1.3 Canonical examples . . . . . . . . . . . . . . 78

5.2 .2 Innerly asymptotically $p$-flat sets . . . . . . . . . . . . . 80 
5.2 .2 .1 Definitions . . . . . . . . . . . . . . . 80

5.2 .2 .2 Remarks and examples . . . . . . . . . . . 80

5.2 .2 .3 Stability results . . . . . . . . . . . . . . 82

5.3 Generating a space through an operator $\ldots \ldots \ldots \ldots$

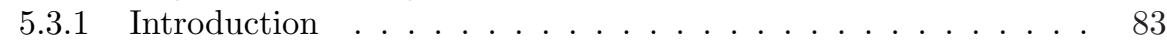

5.3.1.1 Remarks and examples . . . . . . . . . . . 83

5.3 .2 The results . . . . . . . . . . . . . . . . . 85

5.3.2.1 The Asplund setting . . . . . . . . . . . . . . 85

5.3.2.2 The general setting . . . . . . . . . . . 85

5.3 .3 Proofs . . . . . . . . . . . . . . . . . . 87

5.4 Some remarks on long Schauder bases _ . . . . . . . . . . . 94

5.5 Fixing $c_{0}(\Gamma)$ by operators $\ldots \ldots \ldots \ldots \ldots \ldots \ldots$

5.5 .1 Introduction and results . . . . . . . . . . . . . . . . 99

5.5 .2 Proofs . . . . . . . . . . . . . . . . . . 100

6 Appendix 107

6.1 Appendix A . . . . . . . . . . . . . . . . . . . . . . 107

6.2 Appendix B . . . . . . . . . . . . . . . . . . . . . . . 109

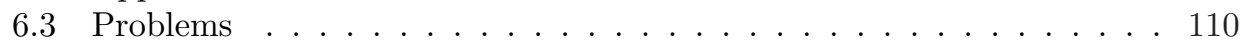

$\begin{array}{ll}\text { References } & 111\end{array}$ 


\section{Introduction, Summary and Notation}

\subsection{Introduction}

This work is presented in order to obtain the PHD degree in Mathematics. It consists of a Memoir, and it develops certain aspects of a subject that was proposed to me by my tutors, Professors Marián Fabian and Vicente Montesinos. Most of the material included we believe is new. Of course, in order to conveniently present it, we also incorporated some preliminaries, some known results (sometimes with new proofs) and some accessory material. Those different levels are carefully differentiated, in order to make it clear to the reader what is what at each stage. Certainly, not all the new material has the same importance, and we tried, as far as possible, to enhance what we think is more relevant. This is why some results wear the label of "theorems", some others of "propositions", "remarks", "lemmata" and the like, in order to give continuity and completeness to the presentation. We try to follow an order such that it makes clear what are the main results. We think that part of the purpose of this Memoir - and likewise others - is to prove that the author is able to elaborate a scientific document, one (by the way an important one) of the many that she would have to prepare along her scientific career. Then, the final coherence of the result is something to be considered, too.

The work evolves, naturally, from the scientific interest of the tutors, something that, needless to say, has been transmitted to us. This can be seen in the list of references at the end of the Memoir. Many among them are authored by the tutors and their group ( [Fab97], [FG88], [FGHZ03], [FGMZ04], [FGZ01], [FHHMPZ01], [FHMZ], [FMZ05], [FMZ04], [FMZ07], [FMZ02], [HLM07], [HMVZ07], [DGZ], [Rych04], [Vand95], [Z03]). The rest reflect their and our interest. We contributed already to the references with three papers on the subject of the Memoir. The first one ([GM]) has been already accepted for publication and is now in press. The second one ([FGZ]) is already submitted, and the third one $([\mathrm{FGM}])$ is almost finished. We want also to mention that some of our results already appeared in book form ([HMVZ07]). 
Our main interest is in nonseparable Banach spaces. This is a wide subject that has already a long history and that continues to be a field of very active research. We focus in this Memoir on some particular topics, like some classes (weakly compactly generated, weakly countably determined, weakly Lindelöf spaces) and the kind of compacta related to them (Eberlein, Gul'ko, Corson). We profit from many previous results in the area. The techniques used are typical of the field (projectional resolutions of the identity and projectional generators - and so decompositions of Banach spaces - , biorthogonal systems - in particular Markushevich basis-) together with more general techniques like weak compactness, duality theory, operator theory, convex analysis, general topology and others.

In some sense, this work must be considered unconcluded. Although we are convinced that the material presented has interest and that it is not trivial, we acknowledge that we were not able to solve some problems and we think that the work can be extended beyond the collection of results presented here. We do not think that this is a demerit. From our point of view, this is a proof, among others that have been mentioned above, that the field is alive, and we wish that we can contribute further to enlarge it in a near future. In this direction, we propose at the end of the Memoir a collection of problems that we were not able to solve, and we suggest some lines of research.

\subsection{Notation}

Along this Memoir we shall use the following notation and conventions. We shall work in the context of real Banach spaces. Then, by the word "Banach space" we always mean a real Banach space, denoted by $(X,\|\cdot\|)$ if the mention of the norm is necessary or just convenient, and by $X$ if this is not the case.

Given a Banach space $(X,\|\cdot\|)$, its dual space is denoted by $X^{*}$, and it is the vector space of all linear and continuous mappings from $X$ into $\mathbb{R}$. Equipped with the dual norm (denoted again $\|\cdot\|)$ it becomes a Banach space. The closed unit ball of $(X,\|\cdot\|)$ is written $B_{X}$, and its unit sphere $S_{X}$.

By slightly abusing the notation, given a subset $W$ of a Banach space $X$ we put $B_{W}:=B_{X} \cap W$ and $S_{W}:=S_{X} \cap W$.

Some notational devices: $X^{*}$ is the topological dual of a normed space $X$, so $X^{* *}$ denotes the bidual space. Every space $X$ is supposed to be canonically embedded in its bidual. Elements in the dual of a Banach space will be denoted by $f$ or by $x^{*}$, according to the situation. We shall stick to the second notation especially in the case when elements to successive duals are needed (and then we shall use $x^{*}, x^{* *}$ and so on). The action of an element $x^{*} \in X^{*}$ on an element $x \in X$ is sometimes denoted by $x^{*}(x)$, sometimes by $\left\langle x, x^{*}\right\rangle$. In order to simplify expressions, we shall write $\sup \left\langle M, x^{*}\right\rangle$ instead of $\sup _{x \in M}\left\langle x, x^{*}\right\rangle$, where $M$ is a (bounded) subset of a space $X$ and $x^{*} \in X^{*}$. We denote by $\operatorname{conv}(S)$ the convex hull of a subset $S$ of a normed 
space $X$, and by $\overline{\operatorname{conv}}(S)$ its closed convex hull. The symbol $\Gamma(S)$ denotes the convex hull of the union $S \cup(-S)$. Finally, $\bar{\Gamma}(S)$ means the closure of $\Gamma(S)$.

Topological spaces will be denoted by $(T, \mathcal{T})$ or something similar, where $T$ is a non-empty set and $\mathcal{T}$ the topology on it. So, for example, if $X$ is a Banach space, $\left(X, w\left(X, X^{*}\right)\right)$ denotes the topological space that is obtained by endowing $X$ with the weak topology on it associated to the dual pair $\left\langle X, X^{*}\right\rangle$. This topology, if there is no risk of misunderstanding, will be denoted in short by $w$, and the topology $w\left(X^{*}, X\right)$ by $w^{*}$. The Mackey topology $\tau\left(X, X^{*}\right)$ is the topology on $X$ of the uniform convergence on the family of all absolutely convex and $w^{*}$-compact subsets of $X^{*}$. Similarly, $\tau\left(X^{*}, X\right)$ is the topology on $X^{*}$ of the uniform convergence on the family of all absolutely convex and $w$-compact subsets of $X$.

Given a Banach space $(X,\|\cdot\|)$, a renorming of the space is the action of constructing on $X$ an equivalent norm, say $\|\cdot \mid\|$, i.e., a norm on $X$ that satisfies $A\|\cdot\| \leq\|\cdot \cdot\| \leq B\|\cdot\|$ for some positive constants $A$ and $B$.

Ordinals and cardinals are widely needed. We use $\omega_{0}$ for the ordinal of the set of natural numbers $\mathbb{N}$, and $\aleph_{0}$ for its cardinal number. The first uncountable ordinal number is denoted by $\omega_{1}$, and the corresponding cardinal number by $\aleph_{1}$. The successor of an ordinal number $\gamma$ is denoted by $\gamma+1$. Every ordinal number $\Gamma$ is identified naturally to the initial segment $[0, \Gamma)$. An initial segment of ordinals is usually described as a long sequence of ordinals.

Along the Memoir, definitions that are new are distinctly presented. For many others we refer to the literature or, if we think that a direct access is needed, we recall them at the proper place.

In general, we shall follow [FHHMPZ01] for all unexplained concepts and for notations.

\subsection{Summary}

Chapter 1 has the following purpose: there is a wide hierarchy (from the more narrow to the more general) of classes of compacta appearing in Functional Analysis. Among the ones in which we are interested we list Eberlein, Talagrand, Gul'ko and Corson compacta. Those classes have been the subject of an intense research in the past and continue to be a very active field nowadays. We noticed that, in "everyday life", compacta do not appear in their pristine olympic perfection; on the contrary, they wear very prosaic clothes, adapted to the unpretentious context in which they live. In other terms, although it is common to describe those families as certain classes of subsets in general product of lines, it is more common that the line reduces to a simple finite (even a two-point) subset and that the index set comes endowed with a (more or less natural) topology. In this, more "accessible" context, we investigate Gul'ko compacta, giving intrinsic sufficient conditions for this property (Proposition 7). We start with the well-known characterization of Gul'ko compacta in the general setting 
of subspaces of products of the unit interval, that we state without proof (Theorem 4). After some preliminaries dealing with adequate families of sets (in order to get compact sets of characteristic functions) we formulate and prove some equivalences (Proposition 7), all of them implying that the family defines a Gul'ko compactum (see Remark 9.2). The simplest of those equivalent conditions states that the elements of the family should be closed. Unfortunately, this set of equivalent conditions does not characterizes the class of Gul'ko compacta among spaces of characteristic functions on some index subset of $\mathbb{R}$. We show this by means of an example after Remark 9 . Even more, Theorem 11, due to Leiderman, gives a condition on an adequate family of subsets of some index set $\Gamma$ to get that the compact set of characteristic functions is not a Gul'ko compactum. The proof of this fact is so elegant that we cannot help but including it here. Example 12 (also due to Leiderman) provides a particular instance of this phenomenon. We end this chapter by giving a result that mimics Gul'ko's characterization (in fact, used by Gul'ko as a definition) of Eberlein compacta in terms of pairings, now for the class of Gul'ko compacta. We get (Theorem 4) that a compact space is Gul'ko if and only if it is paired with a $\mathcal{K}$-countably determined topological space. This uses [Fab97, Theorem 7.1.8].

Chapter 2 deals with the class of non-separable weakly compactly generated Banach spaces and their relatives, focusing mostly into weakly Lindelöf determined (WLD) spaces. Since the beginning of the modern theory of non-separable spaces, it was clear that a certain form of coordinate system was needed in order to give significant descriptions, tools that could handle analytic problems and ways to extend by transfinite induction known behaviors typical of the separable setting. This was provided by the concept of Markushevich basis (M-basis), see Definition 16, projectional resolution of the identity (PRI), see Definition 20, and its embryo, the projectional generator (PG), see Definition 21. In the first part of this chapter (Section 2.1), we give some results on those concepts that will be used as tools subsequently, and not only in this chapter. Some of the results are easy (like Propositions 19, 29 or Corollary 31), and we collect them in order to develop smoothly the subject, some others presented are certainly known (like Proposition 32, but only once Theorem 50 has been proved); for some others (Proposition 17, Lemma 27, Proposition 28, Proposition 29, Proposition 35 , Lemma 40, among others) we presume that this may be the case, too, although we could not find the precise reference in the literature, and finally some (Proposition 30, Proposition 41, Lemma 42 among others) represent an effort to formulate general results that can be applied in several situations.

In the hierarchy of non-separable Banach spaces, and trying to exemplify the existence of "natural" PG's, we start by showing (Proposition 43) that the well-known class of WCG Banach space possess full (and single-valued) PG's. We did not find a proper reference to this (probably well-known) fact in the literature. The proof is neat and uses the Mackey topology in the dual of a Banach space. This is used to prove the known (and important fact) that every WCG has a weakly compact M-basis. All this is done in Subsection 2.2.1.

Subsection 2.2.2 deals with the same problem for the case of WCD Banach spaces. 
We need to acknowledge that a hidden description of a PG in such a class was already present in [DGZ, Section VI.2], and an explicit construction is presented in [Fab97, Proposition 7.2.1]; however, this last approach does not use the very definition of a WCD Banach space - and so makes life a little bit more difficult for the reader. Checking that the proposed function is indeed a PG follows some ideas in [DGZ], although needs extra elaboration. This is done in the proof of Proposition 47. Of course, from this we get two well-known facts: every WCD space is WLD (Corollary 54) and every WCD space has a PRI (Corollary 48).

In Subsection 2.2.3, our departure point was the discovery that the existence of a full projectional generator (see Definition 21), something that always exists in every WLD space, in fact characterizes this class (Theorem 50). It is possible that this result has been known by some of the specialists in the area, since it is not so surprising. However, we were unable to locate an earlier reference. We believe that behind the proof provided here lies an idea that does not lack some originality. For the sake of completeness we formulate this theorem by listing, too, a series of equivalent conditions due to several authors, in order to get a wider picture. We rephrase the proof that a WLD Banach space has a full PG using the ideas in [Fab97, Proposition 8.3.1], somehow streamlining it a little bit. A consequence of our approach (Corollary 55 ) is a simple proof of the (known) fact that the quality of being WLD is hereditary by passing to subspaces, something that until now was proved via the deep fact that the continuous image of a Corson compact space is also Corson. In order to do this, we prove first that every subspace of a WLD Banach space has a Markushevich basis (Remark 52). We use also the (simple) fact that the continuous image of an angelic compactum is also angelic. The use of Markushevich bases in the study of some properties of non-separable Banach spaces has a long tradition. An up-to-date presentation of these techniques appear in the recent book [HMVZ07], where, as a matter of fact, our Theorem 50 appears in full - together with Proposition 43. We also provide a proof of the (known) fact (see [Val91] and [Vand95]) that it is always possible to extend a Markushevich basis from a subspace of a WLD Banach space to the whole space (Corollary 57). We need the fact that in a WLD Banach space, we can always produce a PRI subordinated to a linearly dense subset of the space, countably supporting the dual (see Definition 23). We use here our quite general Proposition 30. After proving a simple fact on WLD Banach spaces (all such spaces are DENS, see Corollary 59), we enlarge, in Theorem 61, some previous information given in [Rych04], and evidence that this theorem really depends on a deep theorem of Valdivia [Val96] about biorthogonal systems and Asplund spaces. The equivalences stated in our Theorem 61 are new, modulo the two aforesaid references. Let us insist in that a (full) PG in the more general class of WLD Banach spaces was explicitly constructed in Theorem 50. A more natural PG in this case is constructed in Remark 53. However, we should not be mislead; this PG comes very indirectly from the concept of WLD Banach space, since it needs the non-trivial fact of the existence of a linearly dense subset of the space countably supporting the dual (and so all the equivalences in Theorem 50). By the way, this linearly dense subset can be taken to 
be an M-basis, something that we shall use quite a few times along this Memoir.

Section 2.3 deals with a subject that has been investigated from the beginning of the theory of WCG Banach spaces: the existence (and the quality) of Markushevich basis in such a class of Banach spaces. It was known soon ([AmLi68]) that every WCG Banach space has a Markushevich basis, even a weakly compact one. However, not all Markushevich basis in a WCG Banach space are weakly compact. The main purpose of this section is to prove, by the technology of PRI's, Theorem 71, an extension of a result of Argyros and Mercourakis [ArgMe] pertaining to all Markushevich bases in a WCG Banach space. The original proof of the version for M-bases (Corollary 73) relies on deep combinatorial techniques. We think interesting that our proof uses only separable projectional resolutions of the identity subordinated to an existing weakly compact Markushevich basis. In fact, the very structure of a Markushevich basis is not needed. The only thing we need is the "bottom part" i.e., the set of vectors of the basis, a linearly dense subset $\Gamma$ of $X$ that has what we call here the Amir-Lindenstrauss property (see Definition 63). In order to develop the techniques needed, we make a short excursion on the Amir-Lindentrauss property, and motivate the ArgyrosMercourakis result by showing that any time a biorthogonal system $\left\{x_{\lambda} ; f_{\lambda}\right\}_{\lambda \in \Lambda}$ has the property that the set $\left\{x_{\lambda} ; \lambda \in \Lambda\right\}$ has the Amir-Lindenstrauss property, then $\left\|\sum_{i=1}^{n} f_{\lambda_{i}}\right\| \rightarrow \infty$ whenever $\lambda_{1}, \lambda_{2}, \cdots \in \Lambda$ is a one-to-one sequence and $n \rightarrow \infty$ (Corollary 68). By the way, we think that a credit for this ideas must go, at least partially, to V. Pták, who proved (see Theorem 69) that a Banach space is reflexive if and only if every countable biorthogonal system in it must have a sequence of partial sums of the functional coefficients whose norms tend to infinity. The original proof of this result is quite involved. We provide a proof that depends on the well-known James' characterization of reflexive Banach spaces. We finish this chapter by showing how the result of Argyros and Mercourakis can be used to check the validity of an example provided by Argyros, example that guaranties that a certain subspace of some WCG $C(K)$ space is not WCG.

Summing up, we improve the result of Argyros and Mercourakis (Corollary 73) to Theorem 71, whose proof uses only a PRI argument, deduce a result of Argyros and Farmaki (Theorem 74) from it, and then a result of Johnson (Theorem 75) and finally make Example 77 of Argyros an application of this result. We think that this simple approach can help to clarify the ideas behind.

Chapter 3 is short and follows somehow the approach used in Chapter 1. Although it has been known for many years that an equivalent rotund (i.e., strictly convex) norm exists on $c_{0}(\Gamma)$ (a result of Day [Day55] later strengthened by Rainwater [Rain69] by proving that the so called "Day's norm" is actually locally uniformly rotund), the proof of this result (and the improved one) still is difficult. Here we provide a simple approach to this matter at the price of reducing a little bit the generality of the result, since we assume that $\Gamma$ is in fact a topological subspace of $\mathbb{R}$. This non-very-dramatic restriction makes life easier and can open the way to a quite general result with a not-too-difficult proof. 
Chapter 4 has its origin in the investigation of a class of Banach spaces whose mere existence as a separate entity was not clear from the very beginning of the theory of nonseparable WCG Banach spaces. We have in mind the class of subspaces of WCG Banach spaces. Until the first proof of the existence of a subspace of a WCG Banach space that was not WCG itself (an example due to Rosenthal [Ros74]) it was an open problem whether the WCG property was hereditary by passing to subspaces. In Chapter 2 we studied another example of this "pathology". In [FMZ04] a characterization of the class of subspaces of WCG Banach spaces was done in terms of a covering property of the closed unit ball of the Banach space, by means of what was called there $\varepsilon$-weakly compact sets. Concerning this, we noticed that this concept may be replaced by a more precise one (that we call $\varepsilon$-weakly self-compactness) and that this concept allows for a better description of the sought property of the closed unit ball. Accordingly, we need to elaborate the associated theory, something that is not merely a transcription of the known facts about $\varepsilon$-weakly compact sets. We provide a separation of both concepts and some sufficient conditions for the second one.

Chapter 5 addresses a simple task: To give intrinsic necessary and sufficient conditions for a Banach space $X$ to be generated by $c_{0}(\Gamma)$ or $\ell_{p}(\Gamma)$ for $p \in(1,+\infty)$. Here, "to be generated" means that there exists a continuous linear mapping from the later space into $X$ with dense image.

The original idea comes from a deep result of Godefroy, Kalton and Lancien characterizing when a certain Banach space is a subspace of $c_{0}$ (more generally, of $c_{0}(\Gamma)$ ). They reencounter a geometrical concept, due to Milman, the asymptotically flatness of the closed unit ball. This time the description is done in the language of Kadec-Klee properties of the norm. It turns out that the use of the modulus of smoothness can describe a similar phenomenon. This was done in [FGHZ03]. However, this approach has a severe drawback: according to Dvoretzky's result, the modulus of smoothness of a Banach space cannot have a power type greater than 2 (see, for example, [DGZ, Section IV.4]). Our approach overcomes this difficulty and gives a full answer to the original question. It relies on the technique of PRI's, a device originally developed by Amir and Lindenstrauss, and we believe it is an interesting application (one more - we presented some others in Chapter 2-among a large panoply) of these. We need to split the treatment into two parts. The first one deals with weakly compactly generated (in short, WCG) Asplund spaces - we are, of course, in the context of WCG spaces, since $c_{0}(\Gamma)$ and $\ell_{p}(\Gamma)$ are such. This initial restriction is motivated by the fact that, in this situation, our PRI gives another PRI on the dual, just by taking the adjoint projections. Since our description of flat sets is done by looking at the behavior of sequences in the dual, this is most convenient. Once this is done, we tackle the general case. A superficial glimpse will conclude that the Asplund case should be embedded in the general one. This is not so. First, the kind of sets involved in the characterization is different (we call them, in the first case, asymptotically $p$-flat, in the second, innerly asymptotically $p$-flat) — although these concepts coincide in the case of the closed unit ball of a Banach space. Second, the absence of a PRI on the 
dual is supplied by a certain Asplund behavior of the innerly asymptotically $p$-flat set itself. At this point, we use Proposition 166, a very recent result in this direction that has been proved in [FMZ07]. This result represents, somehow, a certain localization of the Asplund property, and it suits this situation since we can dispense the whole space of the Asplundness condition.

It follows from our characterization that, indeed, they are not many classes of Banach spaces being $c_{0}(\Gamma)$ or $\ell_{p}(\Gamma)$ generated. This looks as if we were dealing with a very narrow class. This is partially true - and was, in fact, one thing that needed to be clarified. On the other hand, all elements in our class are subspaces of Hilbert generated Banach spaces. This class (Banach spaces that are subspaces of Hilbert generated Banach spaces) has received a lot of attention in the last years, since it coincides with the class of Banach spaces whose dual unit ball, when endowed with the $w^{*}$-topology, is a uniform Eberlein compactum.

In most of our treatment, we restricted ourselves mainly to Banach spaces of density character $\omega_{1}$. This is not such a severe constraint. Most of all nonseparable Banach spaces that are interesting for applications fall down into this category. We are almost sure that our results hold with very mild cardinal restrictions. Already the proofs in our case are quite involved, and to deal with them in the general case will add subtleties masking the important ideas.

A byproduct of our approach is a new proof of a result of Rosenthal on operators from $c_{0}(\Gamma)$ into Banach spaces. A classical result ensures that, in the countable case and if the operator is not weakly compact, then it fixes a copy of $c_{0}$, i.e., there exists a subspace of $c_{0}$, isomorphic to $c_{0}$, on which the operator acts as an isomorphism (we say that the operator "fixes a copy of $c_{0}$ "). Rosenthal proved that the result holds true for any infinite set $\Gamma$ as soon as the image of the vectors of the canonical basis have a norm uniformly bounded below by a positive constant. The original proof used deep results on finitely additive measures and a disjointization lemma. We think that our approach is more transparent, and that it can help to understand other related processes. It is true that we tried to apply the same technique to the $\ell_{\infty}$ case, still with no success. This can be the subject of a later research.

We end by a short chapter (Chapter 6) that we call "Appendix" including three tools that were needed in previous chapters. The first one is a complete proof of a result whose attribution we could not locate but that comes as an exercise in [Woj91]. It is used in Chapter 5. It turns out that the proof that we provide is not so simple (maybe there is an easier approach) and, since the exercise is not proved (there is no even a hint) in the aforesaid reference, we preferred to include the complete proof here. The second tool is the result of Pełczyński that every non-weakly compact continuous linear operator from $c_{0}(\Gamma)$ into a Banach space fixes a copy of $c_{0}(\Gamma)$. We believe that the proof we provide here is simpler than the original, and that it can clarify/improve somehow the result. Then we discuss the concept of strongly WCG. We end the chapter with a list of problems and suggested subjects of research. 


\subsection{A matter of contrast}

Our results have gone, partially, to standard checking process.

1. Results on characterization of WLD Banach spaces by means of full projectional generators were presented at the II Meeting in Functional Analysis MurciaValencia, 2006.

2. We presented a communication at the Jornadas del Departamento de Matemática Pura y Aplicada. Valencia, 2006, with the title: A note on weakly Lindelöf determined Banach spaces.

3. The results mentioned at the precedent number appeared already in book form, precisely in [HMVZ07].

4. Fixing $c_{0}(\Gamma)$ was the subject of a Poster Session at the Jornadas del Departamento de Matemática Pura y Aplicada, Universidad Politécnica de Valencia, in 2007.

5. We presented part of Chapter 5, the one dealing with flat sets, at the congress Function Theory on Infinite-dimensional spaces X, Madrid, December 2007. The results will appear in the Proceedings of the Conference.

6. Our results on flat sets and generation of Banach spaces were presented by V. Montesinos in a plenary lecture at the Spring Conference on Banach Spaces, Paseky, Czech Republic, April 2008.

7. At the same conference we presented our results on the Argyros-Mercourakis construction and Argyros' example (with S. Argyros and S. Mercourakis in the audience). This results have been also presented in the Valencia-Murcia meeting, Alcoy (Alicante), May, 2008.

8. Results on M-bases will be also presented at the CITA meeting, to be held in Valencia, June, 2008.

9. We are preparing a short note on the subject mentioned in the previous number.

10. Our paper $[\mathrm{GM}]$ has been already accepted for publication at the Czech Mathematical Journal.

11. Our paper [FGZ] has been submitted to the Journal of the Australian Mathematical Society. 



\section{Chapter 1}

\section{Gul'ko compacta}

As we mentioned in the previous Summary, this chapter treats the class of Gul'ko compacta in the more familiar setting of families of characteristic functions on a certain non-empty (usually uncountable) set $\Gamma$, i.e., we see the compact set as a subset of $\{0,1\}^{\Gamma}$ endowed with the restriction of the pointwise (i.e., the product) topologythis space will be always endowed with the product topology. This allows to provide a simple sufficient condition for being Gul'ko (Proposition 7). This condition is not necessary (as it is showed by Example 10). Later on we describe a sufficient condition for not being Gul'ko, a result due to Leiderman and Sokolov (Theorem 11), and we exhibit a particular example (due to Leiderman) of a family satisfying LeidermanSokolov assumption (Example 12). We finish this chapter by giving a necessary and sufficient condition for being Gul'ko compactum in terms of pairings (Theorem 13).

We start by giving the definition of a class of compact spaces that is widely used in the theory of Banach spaces.

Definition 1 A compact space is called Gul'ko if the space of continuous real functions on it, endowed with the supremum norm, is a Vašák space.

Let us recall here the definition of the class of Vašák spaces.

Definition 2 A Banach space $X$ is Vašák (also called weakly countably determined, in short WCD) if there exists a sequence $\left(K_{n}\right)$ of $w^{*}$-compact subsets of $X^{* *}$ such that, for every couple $\left(x^{* *}, x\right) \in\left(X^{* *} \backslash X\right) \times X$, there exists $n \in \mathbb{N}$ such that $x^{* *} \notin K_{n}$ and $x \in K_{n}$.

For a thorough account on WCD Banach spaces we refer to [DGZ, Chapter VI] and [Fab97, Chapter 7].

Remark 3 Definition 1 is not the one that appears, for example, in [Fab97, Definition 7.1.7]. There, a compact space $K$ is called $G u l^{\prime} k o$ if the space $C(K)$ of real continuous functions on $K$, endowed with the pointwise topology, is $\mathcal{K}$-countably determined 
([Fab97, Definition 7.1.2]), see the definition after Theorem 13. However, as it is proved in [Fab97, Theorem 7.1.8], both definitions are equivalent. We shall use then as definition the statement of Theorem [Fab97, Theorem 7.1.8]. This is the situation in Definition 1.

An internal characterization of Gul'ko compacta is given by the following statement (see, e.g., [FGMZ04, Theorem 10] ). We shall not prove it. Let us recall that the assumption that a compact space is a subset of some product of intervals is not a restriction at all; indeed, every compact topological space has this property.

Theorem 4 Let $K$ be a compact subspace of $[0,1]^{\Gamma} \cap \Sigma(\Gamma)$. Then $K$ is Gul'ko compactum if and only if the following holds: There exists a sequence $\left(\Gamma_{n}\right)_{1}^{\infty}$ of subsets of $\Gamma$ such that, for every $\gamma \in \Gamma, k \in K$ and $\varepsilon>0$, there is $m \in \mathbb{N}$ such that $\gamma \in \Gamma_{m}$ and $\#\left\{\gamma \in \Gamma_{m} ;|k(\gamma)| \geqslant \varepsilon\right\}<\infty$.

The purpose of this chapter is to investigate Gul'ko compacta in natural settings. It is quite common that the abstract set $\Gamma$ in Theorem 4 has some familiar structure, making the statement more accessible and manageable. The task of detecting the Gul'ko property of a certain compact space turns out to be easier.

Let us start by assuming that $K$ is a subset of $\{0,1\}^{\Gamma}$, for some infinite, usually uncountable set $\Gamma$. Thus every element $k \in K$ is in fact a mapping from $\Gamma$ into $\{0,1\}$, i.e., a characteristic function of some subset of $\Gamma$. We shall always make this identification. It turns out that $K$ corresponds to a family $\mathcal{A}$ of subsets of $\Gamma$. We state a well-known sufficient condition on $\mathcal{A}$ to ensure that $K$ is compact, and, for the sake of completeness, we shall provide a proof that, in fact, the condition suffices for compactness.

Definition 5 A family $\mathcal{A}$ of subsets of $\Gamma$ is called adequate if the following holds:

1. for every $\gamma \in \Gamma,\{\gamma\} \in \mathcal{A}$,

2. given $A \in \mathcal{A}$ and $B \subset A$, then $B \in \mathcal{A}$,

3. given $A \subset \Gamma$ such that for every finite subset $F \subset A$ it holds $F \in \mathcal{A}$, then $A \in \mathcal{A}$.

Then we have

Proposition 6 Let $\mathcal{A}$ be an adequate family of subsets of a non-empty set $\Gamma$. Then the set $K_{\mathcal{A}}:=\left\{\chi_{A} ; A \in \mathcal{A}\right\}$ is compact in $\{0,1\}^{\Gamma}$.

Proof. By Tychonov's theorem, $\{0,1\}^{\Gamma}$ is a compact space. It is then enough to prove that $K_{\mathcal{A}}$ is closed in $\{0,1\}^{\Gamma}$. Let $f \in \overline{K_{\mathcal{A}}}$. Then $f=\chi_{A_{0}}$ for some $A_{0} \subset \Gamma$. Fix a finite subset $F \subset A_{0}$. We can find $g \in K_{\mathcal{A}}$, say $g=\chi_{A}$ for some $A \in \mathcal{A}$, such that $g(\gamma)=f(\gamma)$ for all $\gamma \in F$. If $\gamma \in F$, then $1=\chi_{A_{0}}(\gamma)=f(\gamma)=g(\gamma)=\chi_{A}(\gamma)$, hence $\gamma \in A$. It follows that $F \subset A$ and so $F \in \mathcal{A}$. This happens for every finite subset $F$ of $A_{0}$. Then $A_{0} \in \mathcal{A}$ and so $f \in K_{\mathcal{A}}$. 
Obviously, adequacy is not a necessary condition for compactness (the reader can provide examples of finite subsets of $\{0,1\}^{\Gamma}$ - themselves compacta - that do not satisfy the requirements for adequacy).

We specialize now a little bit more the kind of compacta we shall use. Our set $\Gamma$ will be an uncountable ${ }^{1}$ subset of the real line $\mathbb{R}$. The following proposition lists a number of sufficient (equivalent) conditions for a subset of $\{0,1\}^{\Gamma}$ for such a $\Gamma$ to be Gul'ko compactum (see Remarks 9.2 and 9.3).

Proposition 7 Let $\Gamma$ be an uncountable subset of $\mathbb{R}$. Let $\mathcal{A}$ be an adequate family of subsets of $\Gamma$. Let $K_{\mathcal{A}}:=\left\{\chi_{A} ; A \in \mathcal{A}\right\}$. Then $K_{\mathcal{A}}$ is a compact space and the following properties are equivalent:

(i) Every $A \in \mathcal{A}$ is closed in $\Gamma$.

(ii) For every $A \in \mathcal{A}$ and for every compact subset $C \subset \Gamma$, $\# A \cap C<\aleph_{0}$.

(iii) The assignment $\varphi: \Gamma \rightarrow \Gamma_{\mathcal{A}}$ defined as $\varphi(\gamma)=\{\gamma, *\}$ is usco, where $\gamma \in \Gamma$ and $\Gamma_{\mathcal{A}}:=\Gamma \cup\{*\}$, being $*$ an extra element not in $\Gamma$. The space $\Gamma_{\mathcal{A}}$ carries the topology $\mathcal{T}_{\mathcal{A}}$ defined in the following way: each $\gamma \in \Gamma$ is an isolated point, and a subbasis of neighborhoods of $*$ is $\mathcal{S}(*):=\{\{*\} \cup(\Gamma \backslash A) ; A \in \mathcal{A}\}$ (see Remark 8.1).

(iv) If $\mathcal{B}$ is a basis of the topology of $\Gamma$ then, for every $A \in \mathcal{A}$ and every $\gamma \in \Gamma$, there is $B \in \mathcal{B}$ such that $\gamma \in B$ and $\# A \cap B<\aleph_{0}$.

If one (and then all) of the conditions (i) to (iv) holds, then $K_{\mathcal{A}}$ is a Gul'ko compactum.

Remark 8 1. A system $\{\mathcal{U}(s) ; s \in S\}$ of families $\mathcal{U}(s)$ of subsets of a set $S$ is called a neighborhood system for a topology $\mathcal{T}$ on $S$ if the following properties hold for every $s, s_{0} \in S$ :

(a) $\mathcal{U}(s) \neq \emptyset$ and for every $U \in \mathcal{U}(s)$ we have $s \in U$

(b) If $s \in U_{0} \in \mathcal{U}\left(s_{0}\right)$ then there exists $U \in \mathcal{U}(s)$ such that $U \subset U_{0}$.

(c) For every $U_{1}, U_{2} \in \mathcal{U}(s)$ there exists $U \in \mathcal{U}(s)$ such that $U \subset U_{1} \cap U_{2}$.

\footnotetext{
${ }^{1}$ In view of Theorem 4 , every compact subset $K$ of the topological space $[0,1]^{\Gamma}$, where $\Gamma$ is a countable set, is already Gul'ko compact. Actually, such $K$ is metrizable, so something more precise holds: it is an Eberlein compactum. Let us recall that a compact topological space $K$ is an Eberlein compactum if it is homeomorphic to a weakly compact subset of a Banach space endowed with the restriction of its weak topology. Another (equivalent) description (due to Gul'ko itself) of an Eberlein compactum is the following (see Theorem 13): a compact topological space $K$ is Eberlein if and only if it is paired with another compact topological space $M$. The term paired means that there exists a separately continuous mapping $\phi: K \times M \rightarrow[0,1]$ that separates points of $K$ and $M$. Now, it is clear that, if $K$ is compact and metrizable, it is paired with a (linearly dense) null sequence in $C(K)$, since this space is separable. This sequence, together with $\{0\}$, is a compact space. We shall elaborate on pairings a little bit after the proof of Example 12.
} 
In such a case, for every $s \in S$ the family $\mathcal{U}(s)$ is called a basis of neighborhoods of $s$. The topology $\mathcal{T}$ generated by this system is the family of sets $O$ such that for every $s \in O$ there exists $U \in \mathcal{U}(s)$ such that $U \subset O$.

If a system $\{\mathcal{S}(s) ; s \in S\}$ of families $\mathcal{S}(s)$ of subsets of $S$ is given such that the system $\{\mathcal{U}(s) ; s \in S\}$ is a neighborhood system for a topology $\mathcal{T}$, where $\mathcal{U}(s)$ is the family of finite intersections of the elements in $\mathcal{S}(s)$, for every $s \in S$, we say that $\{\mathcal{S}(s) ; s \in S\}$ is a neighborhood subsystem for a topology $\mathcal{T}$, and each family $\mathcal{S}(s)$ is called a subbasis of neighborhoods of $s$, for every $s \in S$.

Then the system $\{\mathcal{S}(\gamma) ; \gamma \in \Gamma\} \cup \mathcal{S}(*)$, where $\mathcal{S}(\gamma):=\{\gamma\}$ for all $\gamma \in \Gamma$ and $\mathcal{S}(*)$ is given in the statement (iii) of Proposition 7, is a neighborhood subsystem of a topology in $\Gamma \cup\{*\}$. The proof of this is simple: indeed, properties (a), (b) and (c) for the system $\{\mathcal{U}(\gamma) ; \gamma \in \Gamma\} \cup \mathcal{U}(*)$ defined by finite intersections as before are clearly satisfied (property (b) should be checked only in case $s_{0}=*$, and then, if $* \neq s \in \mathcal{U}(*)$ then $s=\gamma$ for some $\gamma \in \Gamma$ and so $\{\gamma\} \subset \mathcal{U}(*))$.

2. Condition (i) in Proposition 7 implies that every $A \in \mathcal{A}$ is discrete, i. e., the topology of $\mathbb{R}$ induces on $A$ the discrete topology. Indeed, every subset of $A$ belongs to $\mathcal{A}$, hence every subset of $A$ is closed. This implies that $A$ carries the discrete topology. The converse is not true: there exists an adequate family $\mathcal{A}$ of subsets of $\mathbb{R}$ such that every $A \in \mathcal{A}$ is discrete and yet not every $A \in \mathcal{A}$ is closed. As an example, take a countable infinite subset $\left\{\gamma_{n} ; n=0,1,2, \ldots\right\}$ of $\mathbb{R}$ such that $\gamma_{n} \rightarrow \gamma_{0}$, and put $\mathcal{A}:=\{\{\gamma\} ; \gamma \in \mathbb{R}\} \cup \mathcal{P}\left(\left\{\gamma_{n}\right\}_{n \in \mathbb{N}}\right)$, where $\mathcal{P}(S)$ denotes the family of all subsets of a set $S$. Then $\mathcal{A}$ is an adequate family ${ }^{2}$. Every element in $\mathcal{A}$ is discrete. However, the set $\left\{\gamma_{n} ; n \in \mathbb{N}\right\}$ is not closed. See also Example 10.

3. Condition (i) in Proposition 7 implies that $\chi_{A}$ belongs to Mercourakis' space

$$
c_{1}(\Gamma):=\{f: \Gamma \rightarrow \mathbb{R} ; \quad \forall \varepsilon>0,\{\gamma \in \Gamma ;|f(\gamma)| \geqslant \varepsilon\} \text { is discrete }\},
$$

for all $A \in \mathcal{A}$. Indeed, assume that for some $A \in \mathcal{A}$ we have $\chi_{\mathcal{A}} \notin c_{1}(\Gamma)$. Then for some $0<\varepsilon<1$, the set $\left\{\gamma \in \Gamma ;\left|\chi_{\mathcal{A}}(\gamma)\right| \geqslant \varepsilon\right\}(=A)$ is not discrete. This is a contradiction (see 2 . in this remark).

Proof of Proposition 7 (i) $\Rightarrow$ (ii). Take $A \in \mathcal{A}$ and assume that, for some compact subset $C \subset \Gamma$, we have $\# A \cap C \geqslant \aleph_{0}$. Then $A \cap C$ has an accumulation point, say $a \in \Gamma$. Let $\left(a_{n}\right)$ be a sequence in $A \cap C$ of distinct points which converges to $a$. The set $\left\{a_{n} ; n \in \mathbb{N}\right\}$ is in $\mathcal{A}$; however, it is not closed, a contradiction.

(ii) $\Rightarrow$ (i) Assume that some $A \in \mathcal{A}$ is not closed in $\Gamma$. Let $a_{0} \in \bar{A} \backslash A$, where $\bar{A}$ denotes the closure of $A$ in $\Gamma$. Since $\mathbb{R}$ is metrizable, we can find a sequence $\left(a_{n}\right)$ in $A$ such

\footnotetext{
${ }^{2}$ Properties (1) and (2) are satisfied, obviously. Assume now that $B \subset \mathbb{R}$ is such that for all $F \subset B$ finite, $F \in \mathcal{A}$. If $B \notin \mathcal{A}, B$ contains more than one point. If all points in $B$ are in $\left\{\gamma_{n} ; n \in \mathbb{N}\right\}$, then $B \in \mathcal{A}$; hence there exists $b \in B$ such that $b \notin\left\{\gamma_{n} ; n \in \mathbb{N}\right\}$. The set $\{b, c\}$, where $c$ is some other point in $B$, is a finite set not in $\mathcal{A}$, a contradiction.
} 
that $a_{n} \rightarrow a_{0}$. The set $C=\left\{a_{n}\right\}_{n \in \mathbb{N}} \cup a_{0}$ is compact and $A \cap C=\left\{a_{n}\right\}_{n \in \mathbb{N}}$, an infinite set, a contradiction.

(i) $\Rightarrow$ (iii) Fix $\gamma_{0} \in \Gamma$. We shall prove that $\varphi$ is usco at $\gamma_{0}$. Take $W \in \mathcal{T}_{\mathcal{A}}$ such that $\left\{\gamma_{0}, *\right\} \in W$. The set $W$ is a neighborhood of $*$, so it contains some $U(*):=$ $\bigcap_{i=1}^{n} A_{i}^{c} \cup\{*\} \in \mathcal{U}(*)$, where $A_{i} \in \mathcal{A}, i=1,2, \ldots, n$, and $A^{c}:=\Gamma \backslash A$ for $A \in \mathcal{A}$. From (i) we know that $A_{i}$ is closed in $\Gamma$, for all $i=1,2, \ldots, n$. If $\gamma_{0} \in \bigcap_{i=1}^{n} A_{i}^{c}$ we are done, since $\bigcap_{i=1}^{n} A_{i}^{c}$ is an open set in $\Gamma$ containing $\gamma_{0}$ and $\varphi\left(\bigcap_{i=1}^{n} A_{i}^{c}\right) \subset W$. Assume, on the contrary, that $\gamma_{0} \notin \bigcap_{i=1}^{n} A_{i}^{c}$. Then the set $I_{1}:=\left\{i \in\{1,2, \ldots, n\} ; \gamma_{0} \in A_{i}\right\}$ is non-empty. Put $I_{2}:=\{1,2, \ldots, n\} \backslash I_{1}$. Then, because each $A_{i}$ is discrete in $\Gamma$ (see Remark 8.2), we have that for each $i \in I_{1}$ there exists an open set $V_{i}$ in $\Gamma$ such that $V_{i} \cap A_{i}=\left\{\gamma_{0}\right\}$ (in particular, $\gamma_{0} \in V_{i}$ for all $i \in I_{1}$ ). If $i \in I_{2}$, put $V_{i}:=A_{i}^{c}$. Then $V_{i}$ is open and again $\gamma_{0} \in V_{i}$. It follows that $V:=\bigcap_{i=1}^{n} V_{i}$ is an open set in $\Gamma$ containing $\gamma_{0}$. We shall prove that $\varphi(V) \subset W$. Indeed, given $\gamma \in V, \gamma \neq \gamma_{0}$, we have $\gamma \in V_{i}$, $i=1,2, \ldots, n$. For $i \in I_{1}$, it follows from $V_{i} \cap A_{i}=\left\{\gamma_{0}\right\}$ that $\gamma \in A_{i}^{c}$. For $i \in I_{2}$, it follows from the definition of $V_{i}$ that again $\gamma \in A_{i}^{c}$. We obtain $\gamma \in \bigcap_{i=1}^{n} A_{i}^{c}$. This proves the upper semicontinuity of $\varphi$ at $\gamma_{0}$. Clearly $\varphi$ is compact-valued.

(iii) $\Rightarrow$ (i) Assume that some $A \in \mathcal{A}$ is not closed in $\Gamma$. Find then $\gamma_{0} \in \bar{A} \backslash A$. We shall prove that $\varphi$ is not usco at $\gamma_{0}$. Let $W$ be the neighborhood of $\varphi\left(\gamma_{0}\right)$ given by $W=\{\Gamma \backslash A\} \cup\{*\}$. For every open neighbourhood $U \subset \Gamma$ of $\gamma_{0}$ we have $U \cap A \neq \emptyset$. Hence $\varphi(U)$ is not included in $W$.

(i) $\Rightarrow$ (iv) Let $\mathcal{B}$ be a neighborhood basis for the topology of $\Gamma$. Fix $A \in \mathcal{A}$ and $\gamma \in \Gamma$. Assume first that $\gamma \notin A$. The set $A^{c}:=\Gamma \backslash A$ is open in $\Gamma$, hence there exists $B \in \mathcal{B}$ such that $\gamma \in B \subset A^{c}$ and we obtain $A \cap B=\emptyset$. Assume now that $\gamma \in A$. We know that $A$ carries on the discrete topology (see Remark 8.2). Therefore, we can find $B \in \mathcal{B}$ such that $B \cap A=\{\gamma\}$.

(iv) $\Rightarrow$ (i) Let $\mathcal{B}$ be a basis for the topology of $\Gamma$. Assume that for some $A \in \mathcal{A}, A$ is not closed. Take $a \in \bar{A} \backslash A$. Let $\left(a_{n}\right)$ be a sequence of points in $A$ such that $a_{n} \rightarrow a$. The set $S:=\left\{a_{n} ; n \in \mathbb{N}\right\}$ is infinite and belongs to $\mathcal{A}$. Take $B \in \mathcal{B}$ such that $a \in B$. Then the set $S \cap B$ is infinite, and this contradicts (iv).

For the proof of the last statement, see Remarks 9.1. and 9.2.

Remark 9 1. From the implications (ii) $\Rightarrow$ (iv) and (iv) $\Rightarrow$ (i) we obtain that, in fact, the equivalence between (iv) and the other statements holds for every basis of the topology of $\Gamma$. So, in particular if it happens for some basis, it happens for every basis.

2. The topological space $\Gamma$, as a subset of $\mathbb{R}$, satisfies the second axiom of countability (i.e., its topology has a countable basis). Fix any such a basis $\left\{\Gamma_{n}\right\}_{n \in \mathbb{N}}$. In particular, we have $\Gamma=\bigcup_{n \in \mathbb{N}} \Gamma_{n}$. From 1. in this remark we have that, for every $A \in \mathcal{A}$ and $\gamma \in \Gamma$, there exists $m \in \mathbb{N}$ such that $\gamma \in \Gamma_{m}$ and $\# A \cap \Gamma_{m}<\aleph_{0}$. In view of Theorem $4, K_{\mathcal{A}}$ is a Gul'ko compactum. 
3. The converse of the statement in 2. in this remark does not always hold. Precisely, there exists an uncountable set $\Gamma \in \mathbb{R}$, an adequate family $\mathcal{A}$ of subsets of $\Gamma$ such that $K_{\mathcal{A}}$ is Gul'ko compactum and conditions (i) to (iv) in Proposition 4 do not hold. This is presented in the following example.

Example 10 We work again with $\Gamma=\mathbb{R}$ and

$$
\mathcal{A}=\{\{\gamma\} ; \gamma \in \mathbb{R}\} \cup \mathcal{P}\left(\left\{\gamma_{n} ; n \in \mathbb{N}\right\}\right),
$$

where $\left(\gamma_{n}\right)$ is a sequence of distinct elements in $\mathbb{R}$ which converges to some element in $\mathbb{R}$, say $\gamma_{0}$, and $\mathcal{P}(S)$ denotes the family of all subsets of a set $S$. This is the same family as that used in Remark 8.2. We saw there that $\mathcal{A}$ is an adequate family, so $K_{\mathcal{A}}$ is a compact subset of $\{0,1\}^{\Gamma}$, although this family does not satisfy the requirements in Proposition 7 (as the set $\left\{\gamma_{1}, \gamma_{2}, \ldots\right\}$ is not closed).

To see that $K_{\mathcal{A}}$ is Gul'ko, we shall use Theorem 4. Put

$$
\begin{gathered}
\Gamma_{n}:=[-n, n], \quad \Delta_{n}:=\left[\gamma_{0}-\frac{2}{n}, \gamma_{0}-\frac{1}{n}\right], \quad \text { and } \\
S_{n}:=\left[\gamma_{0}+\frac{1}{n}, \gamma_{0}+\frac{2}{n}\right], \quad n \in \mathbb{N} .
\end{gathered}
$$

The family $\mathcal{F}:=\left\{\Gamma_{n}, \Delta_{n}, S_{n},\left\{\gamma_{0}\right\} ; n \in \mathbb{N}\right\}$ is a covering of $\mathbb{R}$. Fix $A \in \mathcal{A}$ and $\mathbb{R}$. If $A$ is finite, there is nothing to prove. Otherwise, put $A:=\left\{\gamma_{n} ; n \in M\right\}$ for some infinite set $M \subset \mathbb{N}$. If $\gamma=\gamma_{0}$, then $\gamma \in\left\{\gamma_{0}\right\}$ and obviously $\left\{\gamma_{0}\right\} \cap A$ is finite. If, on the contrary, $\gamma \neq \gamma_{0}$, we can find some $S \in \mathcal{F}$ such that $\gamma \in S$ and $S \cap A$ is finite. By Theorem 4, the compact space $K_{\mathcal{A}}$ is Gul'ko. However, (i) in Proposition 7 does not hold. (It should be noted that our $K_{\mathcal{A}}$ is even an Eberlein compactum.)

Until now, all compact spaces defined by adequate families in our setting were Gul'ko (compacta verifying one of the equivalent conditions in Proposition 7 or the example in Remark 9.2). This is not, by all means, always true. We present now a precise situation where a compact space defined by an adequate family of subsets of some infinite $\Gamma$ in $\mathbb{R}$ is not Gul'ko.

Theorem 11 (Leiderman and Sokolov, [LS84]) Let $\Gamma$ be an uncountable set, and let $\mathcal{A}$ be an adequate family of at most countable subsets of $\Gamma$. Assume that for each infinite set $B \subset \Gamma$ there exists an infinite $A \in \mathcal{A}$ such that $A \subset B$. Then $K_{\mathcal{A}}$ is a compact space that is not Gul'ko.

Proof. Assume $K_{\mathcal{A}}$ is Gul'ko. Then, by Theorem 4, we can find $\Gamma_{n} \subset \Gamma, n \in \mathbb{N}$, such that, for every $A \in \mathcal{A}$ and $\gamma \in \Gamma$ there exists $n \in \mathbb{N}$ such that $\gamma \in \Gamma_{n}$ and $\# A \cap \Gamma_{n}<\aleph_{0}$. We may replace the family $\left\{\Gamma_{n} ; n \in \mathbb{N}\right\}$ by another countable family closed under taking finite intersections. Call this new family again $\left\{\Gamma_{n} ; n \in \mathbb{N}\right\}$. 
For $\gamma \in \Gamma$ let $B_{\gamma}:=\bigcap_{\gamma \in \Gamma_{n}, n \in \mathbb{N}} \Gamma_{n}$. Then, obviously $\gamma \in B_{\gamma}$. We claim that $B_{\gamma}$ is finite. Assume not. Then there exists $A \in \mathcal{A}$ infinite with $A \subset B_{\gamma}$. But then, for all $n$ with $\gamma \in \Gamma_{n}$, we would have $\#\left(A \cap \Gamma_{n}\right)=\# A=\aleph_{0}$, a contradiction.

We claim that there exists $\gamma \in \Gamma$ such that $\Gamma_{n}$ is infinite whenever $n \in \mathbb{N}$ and $\gamma \in \Gamma_{n}$. Assume not. Then, for every $\gamma \in \Gamma$, there exists $n_{\gamma} \in \mathbb{N}$ such that $\gamma \in \Gamma_{n_{\gamma}}$ and $\# \Gamma_{n_{\gamma}}<\aleph_{0}$. Obviously, $\Gamma=\bigcup_{\gamma \in \Gamma} \Gamma_{n_{\gamma}}$, a countable union of finite sets. Then $\Gamma$ should be countable, a contradiction. Thus the claim is proved.

Take one such $\gamma \in \Gamma$. Let $\left\{n \in \mathbb{N} ; \gamma \in \Gamma_{n}\right\}=\left\{n_{1}<n_{2}<n_{3}<\cdots\right\}$. The decreasing sequence $\left(\bigcap_{k=1}^{m} \Gamma_{n_{k}}\right)_{m=1}^{\infty}$ cannot be eventually constant, since $B_{\gamma}$ is finite. Proceed recursively to define a set $B$ in the following way: if $\Gamma_{n_{1}} \backslash \bigcap_{k=1}^{2} \Gamma_{n_{k}} \neq \emptyset$, choose an element in this nonempty set. If, on the contrary, $\Gamma_{n_{1}} \backslash \bigcap_{k=1}^{2} \Gamma_{n_{k}}=\emptyset$, do nothing. If $\bigcap_{k=1}^{2} \Gamma_{n_{k}} \backslash \bigcap_{k=1}^{3} \Gamma_{n_{k}} \neq \emptyset$, choose an element in this nonempty set. In the opposite case, do nothing. Continuing in this way, we define an infinite set $B$ consisting of all the selected points in the former procedure. Find, by hypothesis, some infinite $A \in \mathcal{A}$ such that $A \subset B$. From the very property of $\Gamma_{n}$ 's, there exists $k \in \mathbb{N}$ such that $\gamma \in \Gamma_{n_{k}}$ and $\#\left(\Gamma_{n_{k}} \cap A\right)<\aleph_{0}$. This is, obviously, false and we arrive to a contradiction. Therefore, the compact space $K_{\mathcal{A}}$ is not Gul'ko.

Example 12 (Leiderman) There exists an adequate family $\mathcal{A}$ of subsets of $\Gamma:=$ $[0,1]$ that satisfies the requirements in Theorem 11, and hence the corresponding compact space $K_{\mathcal{A}}$ is not Gul'ko.

Proof. Define the family $\mathcal{A}$ by

$$
\mathcal{A}:=\left\{A \subset \Gamma ; \exists t \in \mathbb{R}, \quad \sum_{\gamma \in A}|t-\gamma| \leqslant 1\right\} .
$$

We shall see that $\mathcal{A}$ is adequate. Conditions (1) and (2) of Definition 5 are obvious. We shall prove that condition (3) holds, too. Let $A$ be a subset of $\Gamma$ such that, for every finite set $F \subset A$, we have $F \in \mathcal{A}$. Let us partially order the family of all finite subsets $\mathcal{F}$ of $A$ by inclusion: $F_{1} \leq F_{2}$ if and only if $F_{1} \subset F_{2}$ for $F_{1}$ and $F_{2}$ in $\mathcal{F}$. Thus the family $\mathcal{F}$ is a partially ordered set directed upwards. To any $F \in \mathcal{F}$ we associate $t_{F}$ in $[0,1]$ verifying $\sum_{\gamma \in F}\left|t_{F}-\gamma\right| \leq 1$. We obtain a net $\left\{t_{F} ; F \in \mathcal{F}, \leq\right\}$. This net has a convergent subnet $\left\{t_{F_{\iota}} ; \iota \in I, \leq\right\}$. Let $t_{0}$ be its limit. We claim that $A$ satisfies $\sum_{\gamma \in A}\left|t_{0}-\gamma\right| \leq 1$ (and thus $A \in \mathcal{A}$ ).

To prove the claim, let $F \in \mathcal{F}$. Let $\# F=n$. Assume that $\sum_{\gamma \in F}\left|t_{0}-\gamma\right|=1+\varepsilon$, for some $\varepsilon>0$. Find $\iota_{0} \in I$ such that $\left|t_{F_{\iota}}-t_{0}\right|<\varepsilon /(2 n)$ for every $\iota \in I, \iota \geq \iota_{0}$. Find then $\iota_{1} \in I, \iota_{1} \geq \iota_{0}$, such that $F \subset F_{\iota_{1}}$. Then $\sum_{\gamma \in F_{\iota_{1}}}\left|\gamma-t_{F_{\iota_{1}}}\right| \leq 1$. We get

$$
1+\varepsilon=\sum_{\gamma \in F}\left|\gamma-t_{0}\right| \leq \sum_{\gamma \in F}\left|\gamma-t_{F_{\iota_{1}}}\right|+\sum_{\gamma \in F}\left|t_{F_{\iota_{1}}}-t_{0}\right|<1+\varepsilon / 2,
$$

a contradiction. The claim is proved and condition (3) in Definition 5 is satisfied. 
Now we shall prove that $\mathcal{A}$ in fact satisfies the conditions of Theorem 11. Obviously, every $A \in \mathcal{F}$ is at most countable. Let $B \subset \Gamma$ be an infinite set. Since $\Gamma$ is bounded, $B$ contains an accumulation point, say $\gamma_{0}$. Then there exists an infinite sequence $\left(\gamma_{n}\right)$ in $B$ such that $\gamma_{n} \rightarrow \gamma_{0}$. Select a subsequence $\left(\gamma_{n_{k}}\right)_{k \in \mathbb{N}}$ in such a way that $\left|\gamma_{n_{k}}-\gamma_{0}\right| \leqslant 2^{-k}$ for $k=1,2, \ldots$ Put $\hat{\gamma}_{k}:=\gamma_{n_{k}}$ for all $k \in \mathbb{N}$. Thus, $\sum_{k=1}^{\infty}\left|\hat{\gamma}_{k}-\gamma_{0}\right| \leqslant$ $\sum_{k=1}^{\infty} 2^{-k} \leqslant 1$. It follows that $\left\{\hat{\gamma}_{k} ; k \in \mathbb{N}\right\} \in \mathcal{A}$. This proves that, for every infinite set $B \subset \Gamma$, there exists an infinite $A \in \mathcal{A}$ such that $A \subset B$. It is enough to apply Theorem 11 to conclude that $K_{\mathcal{A}}$ is not Gul'ko.

A characterization of Eberlein compacta in terms of pairings is due to Gul'ko (see, for example, $[\mathrm{Na}]$ and $[\mathrm{NaWh} 86])$. Given two topological spaces $\mathbb{X}, \mathbb{Y}$, we say that they form a pairing if there exists a separately continuous mapping $f: \mathbb{X} \times \mathbb{Y} \rightarrow[-1,1]$ such that $\mathbb{X}$ separates points of $\mathbb{Y}$, i.e., if $f\left(x_{1}, y\right)=f\left(x_{2}, y\right)$ for every $y \in \mathbb{Y}$, then $x_{1}=x_{2}$, and $\mathbb{Y}$ separates points of $\mathbb{X}$, i.e., if $f\left(x, y_{1}\right)=f\left(x, y_{2}\right)$ for every $x \in \mathbb{X}$, then $y_{1}=y_{2}$. We have the following result.

Theorem 13 (Gul'ko, see, e.g., [NaWh86]) Let $K$ be a compact space. Then $K$ is an Eberlein compactum if and only if it is paired with another compact space.

It is possible to state a similar result characterizing Gul'ko compacta in terms of pairings. Recall that a topological space $\mathbb{Y}$ is called $\mathcal{K}$-countably determined $(\mathcal{K}$-c.d.) if $\mathbb{Y}$ is completely regular, i.e., $\mathbb{Y}$ is a subspace of a compact space $K$, and there exists a sequence $\left(A_{n}\right)_{n=1}^{\infty}$ of closed subspaces of $K$ such that, for every $y \in \mathbb{Y}$ and $k \in K \backslash \mathbb{Y}$ there exists $n \in \mathbb{N}$ such that $y \in A_{n}$ and $k \notin A_{n}$.

Theorem 14 Let $K$ be a compact space. Then $K$ is a Gul'ko compactum if and only if $K$ is paired with a $\mathcal{K}$-countably determined topological space.

Proof. By [Fab97, Theorem 7.1.8], if $K$ is a Gul'ko compactum, then there exists a $\mathcal{K}$ c.d. subspace of $(C(K), p)$, say $\mathbb{Y}$, which separates the points of $K$, i.e., if $k_{1}, k_{2} \in K$ are distinct, there is $f \in \mathbb{Y}$ such that $f\left(k_{1}\right) \neq f\left(k_{2}\right)$. Here, $p$ denotes the topology on $C(K)$ of the pointwise convergence.

We endow $C(K)$ with the norm $\|\cdot\|_{\infty}$. Then $\left(C(K),\|\cdot\|_{\infty}\right)$ becomes a Banach space. Let $M(K)$ be its dual. Then $K$ can be identified (topologically) to a subset of the unit sphere of $M(K)$, endowed with the $\sigma(M(K), C(K))$-topology. Let $\phi: \mathbb{R} \rightarrow(-1,1)$ be a homeomorphism. Let us define $\langle f, k\rangle:=\phi(f(k))$ for all $f \in \mathbb{Y}$ and $k \in K$. Then $\langle\cdot, \cdot\rangle$ is a separately continuous mapping from $\mathbb{Y} \times K$ into ]0,1[ defining a pairing, since $\mathbb{Y}$ separates points of $K$ and, obviously, $K$ separates points of $\mathbb{Y}$.

Conversely, assume that $K$ is a compact space and that $\mathbb{Y}$ is a $\mathcal{K}$-c.d. topological space paired with $K$. Let $\Phi: \mathbb{Y} \times K \rightarrow[-1,1]$ be the mapping defining the pairing. Then we can define a mapping $\Psi: \mathbb{Y} \rightarrow C(K)$ by

$$
\Psi(y)(k):=\Phi(y, k), \quad k \in K, \quad y \in \mathbb{Y} .
$$

Obviously $\Psi(y) \in C(K), \forall y \in \mathbb{Y}$. Moreover, $\Psi$ is continuous when $C(K)$ is endowed with the pointwise topology $p$; this follows from the separate continuity of $\Phi$. By 
[Fab97, Theorem 7.1.3 (i)], $\Psi(\mathbb{Y})$ is a $\mathcal{K}$-c.d. subspace of $(C(K), p)$. Moreover, $\Psi(\mathbb{Y})$ separates points of $K$. We can use now (iii) $\Rightarrow$ (i) in [Fab97, Theorem 7.1.8] to conclude that $K$ is a Gul'ko compactum. 



\section{Chapter 2}

\section{WCG Banach spaces and their relatives}

In this chapter we analyze several features of WCG, WCD and WLD Banach spaces, and the projectional resolutions of the identity defined on them. In the development, we shall use the root concept named a projectional generator. A sketch of the content was outlined in the Introduction. A natural ingredient of many of our proofs is a Markushevich basis. The last part of the chapter deals with biorthogonal systems in WCG Banach spaces.

\section{$2.1 \quad$ Some tools}

In this instrumental section we recall some tools that are needed in the rest of the Memoir. There is a well-established theory as it can be found, for example, in [Fab97, Chapter 6], or in [HMVZ07, Section 3.4]. Here we review some definitions and complete the current information spread along several sources. The extra features in some of the results that we present turn to be crucial in some of the later developments. We claim, also, that the information provided here unifies and clarifies many results in this area.

The first ingredient in our discussion is the concept of Markushevich basis, that we shall define below.

Definition 15 Let $X$ a Banach space. A set $\left\{x_{\gamma} ; x_{\gamma}^{*}\right\}_{\gamma \in \Gamma}$ in $X \times X^{*}$ is called a biorthogonal system if $\left\langle x_{\gamma}, x_{\beta}^{*}\right\rangle=\delta_{\gamma, \beta}$ for every $\gamma, \beta \in \Gamma$. A set $\left\{x_{\gamma} ; \gamma \in \Gamma\right\} \subset X$ is called minimal if there exists a set $\left\{x_{\gamma}^{*} ; \gamma \in \Gamma\right\} \subset X^{*}$ such that $\left\{x_{\gamma} ; x_{\gamma}^{*}\right\}_{\gamma \in \Gamma}$ is a biorthogonal system.

Definition $16 A$ Markushevich basis (in short, M-basis) in $X \times X^{*}$, is a biorthogonal system $\left\{x_{\gamma} ; x_{\gamma}^{*}\right\}_{\gamma \in \Gamma}$ such that $\left\{x_{\gamma}\right\}_{\gamma \in \Gamma}$ is fundamental -i.e., linearly dense in $X-$ and $\left\{x_{\gamma}^{*}\right\}_{\gamma \in \Gamma}$ is total -i.e., $w^{*}$-linearly dense in $X^{*}$. 
The following simple proposition is probably known. However, we did not find any explicit statement in the literature, although it was mentioned, for example, in [HMVZ07, Corollary 4.19]. So we state and prove it here. We shall use it later on (in Proposition 35 and then in Chapter 5). Recall, again from Definition 16, the concepts of fundamentality and totality.

Proposition 17 The cardinality of any fundamental minimal system in a Banach space $X$ coincides with its density.

Proof. Let $\left\{x_{\gamma}\right\}_{\gamma \in \Gamma}$ be a fundamental minimal system in $X$ and let $D \subset X$ be a dense subset with $\# D=\operatorname{dens} X$. Given $d \in D$ there exists a countable set $\Gamma_{d} \subset \Gamma$ such that $d \in \overline{\operatorname{span}}\left\{x_{\gamma}: \gamma \in \Gamma_{d}\right\}$. Then $D \subset \overline{\operatorname{span}}\left\{x_{\gamma}: \gamma \in \Gamma_{d}, d \in D\right\}$, hence $X=\overline{\operatorname{span}}\left\{x_{\gamma} ; \gamma \in \Gamma_{d}, d \in D\right\}$. Observe that $\# \bigcup_{d \in D} \Gamma_{d}=\# D$. If $\gamma_{0} \in \Gamma \backslash \bigcup_{d \in D} \Gamma_{d}$, we have $x_{\gamma_{0}} \notin \overline{\operatorname{span}}\left\{x_{\gamma}: \gamma \in \Gamma_{d}, d \in D\right\}=X$, a contradiction. Then $\bigcup_{d \in D} \Gamma_{d}=\Gamma$ and we get $\# \Gamma=\# D(=\operatorname{dens} X)$.

Definition 18 Let $X$ be a Banach space. An M-basis $\left\{x_{i} ; x_{i}^{*}\right\}$ in $X \times X^{*}$ is called shrinking if $\overline{\operatorname{span}}\|\cdot\|\left\{x_{i}^{*} ; i \in I\right\}=X^{*}$.

We shall need in the sequel the following simple fact about biorthogonal systems.

Proposition 19 Let $X$ be a Banach space and $\left\{x_{i} ; x_{i}^{*}\right\}_{i \in I}$ an $M$-basis in $X \times X^{*}$. Let $J \subset I$ be a non-empty set. Then $\left\{x_{j} ; q x_{j}^{*}\right\}_{j \in J}$ is an M-basis in $Y \times Y^{*}$, where $Y:=\overline{\operatorname{span}}\left\{x_{j} ; j \in J\right\}$ and $q: X^{*} \rightarrow Y^{*}$ is the canonical quotient mapping. If $\left\{x_{i} ; x_{i}^{*}\right\}_{i \in I}$ is shrinking, so it is $\left\{x_{j} ; q x_{j}^{*}\right\}_{j \in J}$.

Proof. Certainly, $\left\{x_{j} ; q x_{j}^{*}\right\}_{j \in J}$ is a fundamental biorthogonal system in $Y \times Y^{*}$. It is clear that $q x_{i}^{*}=0$ for every $i \in I \backslash J$. Then, $\left\{q x_{i}^{*} ; i \in I\right\}=\left\{q x_{j}^{*} ; j \in J\right\} \cup\{0\}$. Since $q$ is $w^{*}-w^{*}$-continuous, the set $\left\{q x_{j}^{*} ; j \in J\right\}$ is $w^{*}$-linearly dense in $Y^{*}$.

Assume now that $\left\{x_{i} ; x_{i}^{*}\right\}_{i \in I}$ is shrinking. Since $q$ is also $\|\cdot\|-\|\cdot\|$-continuous, we get that $\left\{q x_{j}^{*} ; j \in J\right\}$ is $\|\cdot\|$-linearly dense in $Y$, so $\left\{x_{j} ; q x_{j}^{*}\right\}_{j \in J}$ is also shrinking.

The possibility of introducing a "system of coordinates" in a separable (or nonseparable) Banach space effectively reduces some of the arguments associated to certain constructions to analytic computations. In the case of a separable Banach space, a Schauder basis (if there exists one, which is not always the case) or, more generally, a countable M-basis (which always exists by the classical Markushevich theorem, see, e.g., [FHHMPZ01, Theorem 6.41]) do the job. In the non-separable case, an M-basis is not always available, although the most natural non-separable Banach spaces posses one. For more information, see, e.g., [HMVZ07], where an up-to-dated presentation of the theory of biorthogonal systems is done. A related "coordinatewise" structure is a projectional resolution of the identity (a long sequence of norm-one projections somehow "splitting" the space, see Definition 20). A device to produce in a natural way such a structure is a projectional generator (see Definition 21). 
We recall here the definition of a projectional resolution of the identity (in short, PRI). A good account on this concept and the theory related can be found in [Fab97, Chapter 6]. It is mentioned there that first PRI's were constructed by J. Lindenstrauss [L1], [L2], see also [AmLi68]. Similar ideas are also used by D.G. Tacon [T70] who has found a PRI on certain dual spaces. An elegant topological method for obtaining the PRI is due to S. P. Gul'ko [Gul79], see also [NaWh86]. Some of his ideas, translated to the language of Banach spaces, can be found in M. Valdivia [Val88].

Definition 20 Let $(X,\|\cdot\|)$ be a nonseparable Banach space. Let $\mu$ be the first ordinal with cardinal dens $(X)$. A "long sequence" $\left(P_{\alpha}\right)_{\omega_{0} \leq \alpha \leq \mu}$ of linear projections on $X$ such that $P_{\omega_{0}}=0$ and $P_{\mu}=I d_{X}$, is called $a$ projectional resolution of the identity $(\mathrm{PRI})$ on $(X,\|\cdot\|)$ if the following holds.

(i) $\left\|P_{\alpha}\right\|=1$ for all $\alpha \in\left(\omega_{0}, \mu\right]$.

(ii) $P_{\alpha} P_{\beta}=P_{\min \{\alpha, \beta\}}$ for all $\alpha \in\left[\omega_{0}, \mu\right]$.

(iii) dens $\left(P_{\alpha}(X)\right) \leq \# \alpha$ for all $\alpha \in\left[\omega_{0}, \mu\right]$.

(iv) For every $x \in \bar{X}$, the mapping $\alpha \mapsto P_{\alpha}(x)$ from $\left[\omega_{0}, \mu\right]$ into $X$ is continuous.

Let us recall that, given a Banach space $(X,\|\cdot\|)$ and $0<\lambda \leq 1$, a subset $N$ of $X^{*}$ is called $\lambda$-norming if $\lambda\|x\| \leq \sup \left\{\left|\left\langle x, x^{*}\right\rangle\right| ; x^{*} \in N \cap B_{X^{*}}\right\}(\leq\|x\|)$ for every $x \in X$. The subset $N$ is called norming if it is $\lambda$-norming for some $\lambda>0$.

Definition 21 Let $(X,\|\cdot\|)$ be a nonseparable Banach space. A couple $(N, \Phi)$ is called a projectional generator (in short, a PG) for $X$ if $N$ is a 1-norming subset of $X^{*}$ such that $\operatorname{span}_{\mathbb{Q}}(N)$, the linear span of $N$ with rational coefficients, coincides with $N$, and $\Phi: N \rightarrow 2^{X}$ is an at most countably-valued mapping such that, for all $W \subset N$ with $\operatorname{span}_{\mathbb{Q}}(W)=W$, we have

$$
(\Phi(W))^{\perp} \cap \bar{B}_{W} w^{*}=0 .
$$

It is well known that every Banach space with a PG has a PRI (see, e.g., [Fab97, Proposition 6.1.7]). A good account on this concept and the way how a projectional resolution of the identity is constructed from it can be found, for example, in [Fab97, $\S 6.3]$. In particular, and regarding the history of this concept, it is mentioned there that an involved form of a projectional generator can be traced back to K. John and V. Zizler [JZ74], [JZ74-b]. Projectional generators in a more explicit form can be found in the papers [Fab87], [Fab87-b] and [FG88]. Finally J. Orihuela and M. Valdivia introduced in [OV89] the concept of projectional generator in order to unify the constructions from [FG88], [Val90], and [Val88]. Our definition is a slight variant of that from [OV89].

If the PG belongs to a special class (what we shall call full PG, see Definition 22) then the PRI defined from it enjoys some extra properties (see Proposition 35). This will be used in Chapter 5 .

Projectional generators appear in a natural way: for example, it is easy to prove that, in a weakly compactly generated (in short, WCG) Banach space $X$ (i.e., $X$ possesses 
a linearly dense weakly compact subset $K)$, the couple $\left(X^{*}, \Phi\right)$, where $\Phi\left(x^{*}\right)$ is a certain element of $K$ where $x^{*}$ attains its supremum on $K$, is a PG. We illustrate this fact in Proposition 43. Another natural (and more general) class of non-separable Banach spaces, the one introduced by the Vašák (i.e., weakly countably determined, WCD in short, see Definition 2) Banach spaces, also have a PG defined in a simple way (see Proposition 47). A class that is yet more general than that of Vašák spaces and that still has a PG is the class of weakly Lindelöf determined (WLD, in short) Banach spaces (see Definition 49). Although the existing PG in the WCG and WCD cases is introduced naturally (i.e., with ingredients from the very definition), the PG in the WLD case is defined through a more elaborated detour (see Remark 53). This three instances of PG's - that will be considered in greater detail in Section 2.2 are particular examples of the following class.

Definition 22 Given a Banach space $X$, we say that a $P G(N, \Phi)$ for $X$ is full if $N=X^{*}$.

In Subsection 2.2.3 we shall characterize Banach spaces having a full PG. We can advance that those are precisely the WLD ones (see Theorem 50).

Definition 23 Let $\langle E, F\rangle$ be a dual pair. Let $F_{0} \subset F$ be a non-empty subset of $F$. $A$ set $E_{0} \subset E$ is said to countably support $F_{0}$ if

$$
\operatorname{supp}_{E_{0}}(f):=\left\{e \in E_{0} ;\langle e, f\rangle \neq 0\right\}
$$

is a countable set for every $f \in F_{0}$.

Remark 24 A well known result of Amir and Lindenstrauss [AmLi68] says that, for every WCG Banach space, $X$ there exists an M-basis $\left\{x_{\gamma} ; x_{\gamma}^{*}\right\}_{\gamma \in \Gamma}$ in $X \times X^{*}$ with the following property: given $x^{*} \in X^{*}$ and $\varepsilon>0$ the set $\left\{\gamma \in \Gamma ;\left|\left\langle x_{\gamma}, x^{*}\right\rangle\right|>\varepsilon\right\}$ is finite (we provide a proof in Remark 45). In Definition 63 we name this property, and we prove in Proposition 66 that it implies that the set $\left\{x_{\gamma} ; \gamma \in \Gamma\right\}$, together with $\{0\}$, is weakly compact and countably supports $X^{*}$.

Remark 25 It is a simple fact that, if $X$ is a Banach space, every fundamental biorthogonal system $\left\{x_{\gamma} ; x_{\gamma}^{*}\right\}_{\gamma \in \Gamma}$ in $X \times X^{*}$ has the property that $\left\{x_{\gamma}^{*} ; \gamma \in \Gamma\right\}$ countably supports $X$. Indeed, given $x \in X$, there exists a countable set $\Gamma_{0} \subset \Gamma$ such that $x \in \operatorname{span}\left\{x_{\gamma} ; \gamma \in \Gamma_{0}\right\}$. Then $\left\langle x, x_{\gamma}^{*}\right\rangle=0$ for every $\gamma \notin \Gamma_{0}$.

The following concept will appear quite often in this Memoir.

Definition 26 Let $(X,\|\cdot\|)$ be a Banach space with a $P R I\left(P_{\alpha}\right)_{\omega_{0} \leq \alpha \leq \mu}$. We say that a set $G \subset X$ is subordinated to the PRI if, for every $\gamma \in G$ and every $\alpha \in\left[\omega_{0}, \mu\right]$, we have $P_{\alpha}(\gamma) \in\{\gamma, 0\}$. We shall also say, in this case, that the PRI is subordinated to $G$. 
Geometrically, this means that our set $G$ lies in the union of the difference spaces, i.e., $G \subset \bigcup_{\omega_{0} \leq \alpha<\mu}\left(P_{\alpha+1}-P_{\alpha}\right) X$.

We shall use the following simple lemma.

Lemma 27 Let $(X,\|\cdot\|)$ be a Banach space and $\left(P_{\alpha}\right)_{\omega_{0} \leq \alpha \leq \mu}$ a PRI on it. Let $\Gamma \subset X$ and $\Delta \subset X^{*}$ be two sets, the first one subordinated to $\left(P_{\alpha}\right)_{\omega_{0} \leq \alpha \leq \mu}$, the second to $\left(P_{\alpha}^{*}\right)_{\omega_{0} \leq \alpha \leq \mu}$. Then $\Gamma \subset \bigcup_{\omega_{0} \leq \alpha<\mu} P_{\alpha} X$ and $\Delta \subset \bigcup_{\omega_{0} \leq \alpha<\mu} P_{\alpha}^{*} X^{*}$.

Proof. Fix $x \in \Gamma$. Assume that for no $\alpha \in\left[\omega_{0}, \mu\right)$ we have $x \in P_{\alpha} X$. Then, since $\Gamma$ is subordinated to $\left(P_{\alpha}\right)_{\omega_{0} \leq \alpha \leq \mu}$, we have $P_{\alpha} x=0$ for every $\alpha \in\left[\omega_{0}, \mu\right)$. But this implies, by (iv) in Definition 20 , that $x=0$, contradicting the assumption.

The statement about $\Delta$ is proved similarly; it is enough to observe that given $x^{*} \in X^{*}$, the net $\left(P_{\alpha}^{*} x^{*}\right)_{\omega_{0} \leq \alpha<\mu}$ is $w^{*}$-convergent to $x^{*}$.

The following result is somehow expected.

Proposition 28 Assume that a Banach space $(X,\|\cdot\|)$ has a PRI $\left(P_{\alpha}\right)_{\omega_{0} \leq \alpha \leq \mu}$ and an $M$-basis $\left\{x_{i} ; x_{i}^{*}\right\}_{i \in I}$ such that the set $\left\{x_{i} ; i \in I\right\}$ is subordinated to this PRI. Put $I_{\alpha}:=\left\{i \in I ; x_{i} \in P_{\alpha} X\right\}$ for every $\omega_{0} \leq \alpha \leq \mu$. Then we have

(i) $\bigcup_{\omega_{0} \leq \alpha<\mu} I_{\alpha}=I$,

(ii) $\left\{i \in I ; x_{i}^{*} \in P_{\alpha}^{*} X^{*}\right\}=I_{\alpha}$ for every $\alpha \in\left[\omega_{0}, \mu\right]$, and

(iii) $\left\{x_{i}^{*} ; i \in I\right\}$ is subordinated to $\left(P_{\alpha}^{*}\right)_{\omega_{0} \leq \alpha \leq \mu}$.

Proof. (i) follows from Lemma 27.

(ii) Fix any $\alpha \in\left[\omega_{0}, \mu\right)$. Assume first that $i \in I_{\alpha}$. Then

$$
\left\langle x_{j}, P_{\alpha}^{*} x_{i}^{*}\right\rangle=\left\langle P_{\alpha} x_{j}, x_{i}^{*}\right\rangle= \begin{cases}\left\langle x_{j}, x_{i}^{*}\right\rangle, & \text { if } j \in I_{\alpha}, \\ 0=\left\langle x_{j}, x_{i}^{*}\right\rangle, & \text { if } j \notin I_{\alpha} .\end{cases}
$$

It follows that $P_{\alpha}^{*} x_{i}^{*}=x_{i}^{*}$. If $i \notin I_{\alpha}$,

$$
\left\langle x_{j}, P_{\alpha}^{*} x_{i}^{*}\right\rangle=\left\langle P_{\alpha} x_{j}, x_{i}^{*}\right\rangle= \begin{cases}0, & \text { if } j \notin I_{\alpha} \\ 0, & \text { if } j \in I_{\alpha}\end{cases}
$$

and hence $P_{\alpha}^{*} x_{i}^{*}=0$.

(iii) To prove that the set $\left\{x_{i}^{*} ; i \in I\right\}$ is subordinated to $\left(P_{\alpha}^{*}\right)_{\omega_{0} \leq \alpha \leq \mu}$, it is enough to observe, from the proof of (ii), that given $i \in I$ we have only two possibilities: either $P_{\alpha}^{*} x_{i}^{*}=x_{i}^{*}$ or $P_{\alpha}^{*} x_{i}^{*}=0$.

Proposition $29 \operatorname{Let}(X,\|\cdot\|),\left(P_{\alpha}\right)_{\omega_{0} \leq \alpha \leq \mu},\left\{x_{i} ; x_{i}^{*}\right\}_{i \in I}$ and $\left(I_{\alpha}\right)_{\omega_{0} \leq \alpha \leq \mu}$ be as in Proposition 28. Then, for every $\alpha \in\left[\omega_{0}, \mu\right]$, the system $\left\{x_{i} ; x_{i}^{*} \uparrow P_{\alpha} X\right\}_{i \in I_{\alpha}}$ is an $M$ basis in $P_{\alpha} X \times\left(P_{\alpha} X\right)^{*}$.

Proof. Recall that $\left(P_{\alpha} X\right)^{*}$ is isometrically isomorphic to $P_{\alpha}^{*} X^{*}$ for every $\alpha \in\left[\omega_{0}, \mu\right]$. Since $\left\{P_{\alpha} x_{i} ; i \in I\right\}=\left\{x_{i} ; i \in I_{\alpha}\right\} \cup\{0\}$, we get that $P_{\alpha} X=\overline{\operatorname{span}}\left\{x_{i} ; i \in I_{\alpha}\right\}$. It is enough to apply Proposition 19. 
The following result provides a tool that will be used frequently along this Memoir. From our point of view it is the most general statement regarding PRI's on the class of Banach spaces having a full PG. The proof follows the one of [FGMZ04, Proposition 1], adding some extra features.

Proposition 30 Let $(X,\|\cdot\|)$ be a nonseparable Banach space admitting a full PG. Let $M_{1}, M_{2}, \ldots$ be an at most countable family of bounded closed and absolutely convex subsets in $X$. Let $\Gamma \subset B_{X}$ be a set which countably supports $X^{*}$ and let $\Delta \subset B_{X^{*}}$ be a set which countably supports $X$. Then there exists a PRI $\left(P_{\alpha}\right)_{\omega_{0} \leq \alpha \leq \mu}$ on $X$ such that $P_{\alpha}\left(M_{n}\right) \subset M_{n}$ for every $n \in \mathbb{N}, \Gamma$ is subordinated to $\left(P_{\alpha}\right)_{\omega_{0} \leq \alpha \leq \mu}$ and $\Delta$ is subordinated to $\left(P_{\alpha}^{*}\right)_{\omega_{0} \leq \alpha \leq \mu}$.

Proof. Denote $M_{0}=B_{X}$. Let $\Phi_{0}: X^{*} \rightarrow 2^{X}$ be a projectional generator on $X$. Put

$$
\Phi\left(x^{*}\right)=\Phi_{0}\left(x^{*}\right) \cup\left\{\gamma \in \Gamma ;\left\langle\gamma, x^{*}\right\rangle \neq 0\right\}, \quad x^{*} \in X^{*} .
$$

Clearly, $\Phi$ is also a projectional generator. For $n \in \mathbb{N} \cup\{0\}$ and $m \in \mathbb{N}$ let $\|\cdot\|_{n, m}$ be the Minkowski functional of the set $M_{n}+\frac{1}{m} B_{X}$; this will be an equivalent norm on $X$. We shall use a standard back-and-forth argument, see, e.g., [F, Section 6.1]. For every $x \in X$ we find a countable set $\Psi_{0}(x) \subset X^{*}$ such that

$$
\|x\|_{n, m}=\sup \left\{\left\langle x, x^{*}\right\rangle ; x^{*} \in \Psi_{0}(x) \text { and }\left\|x^{*}\right\|_{n, m}^{*} \leq 1\right\}
$$

for every $n \in \mathbb{N} \cup\{0\}$ and $m \in \mathbb{N}$. Put

$$
\Psi(x):=\Psi_{0}(x) \cup\{\delta \in \Delta ;\langle x, \delta\rangle \neq 0\}, x \in X .
$$

Thus we defined $\Psi: X \rightarrow 2^{X^{*}}$. Note that we still have

$$
\|x\|_{n, m}=\sup \left\{\left\langle x, x^{*}\right\rangle ; x^{*} \in \Psi(x) \text { and }\left\|x^{*}\right\|_{n, m}^{*} \leq 1\right\}
$$

for every $n \in \mathbb{N} \cup\{0\}, m \in \mathbb{N}$ and $x \in X$.

For the construction of projections $P_{\alpha}: X \rightarrow X$ we shall need the following Claim. Let $\aleph<$ dens $X$ be any infinite cardinal and consider two nonempty sets $A_{0} \subset$ $X, B_{0} \subset X^{*}$, with $\# A_{0} \leq \aleph, \# B_{0} \leq \aleph$. Then there exists sets $A_{0} \subset A \subset X, B_{0} \subset$ $B \subset X^{*}$ such that $\# A \leq \aleph, \# B \leq \aleph, \bar{A}, \bar{B}$ are linear and $\Phi(B) \subset A, \Psi(A) \subset B$. In order to prove this, put $A=\bigcup_{n=1}^{\infty} A_{n}, B=\bigcup_{n=1}^{\infty} B_{n}$, where the sets

$$
A_{n}=\operatorname{sp}_{Q}\left(A_{n-1} \cup \Phi\left(B_{n-1}\right)\right), \quad B_{n}=\operatorname{sp}_{Q}\left(B_{n-1} \cup \Psi\left(A_{n}\right)\right), \quad n=1,2, \ldots,
$$

are defined inductively. Then it is easy to verify all the proclaimed properties of the sets $A$ and $B$.

Having the sets $A, B$ constructed, we observe that $A^{\perp} \cap \overline{B \cap B_{X^{*}}} w^{*} \subset \Phi(B)^{\perp} \cap$ $\overline{B \cap B_{X^{*}}} w^{*}=\{0\}$. Therefore [HMVZ07, Lemmata $3.33,3.34$ ] yield a linear projection 
$P: X \rightarrow X$, with $P X=\bar{A}, P^{-1}(0)=B_{\perp}$, and $P^{*} X^{*}=\bar{B}^{w^{*}}$, and such that $\|P\|_{n, m}=1$ for every $n \in \mathbb{N} \cup\{0\}$ and $m \in \mathbb{N}$. Then

$$
P M_{n} \subset \bigcap_{m=1}^{\infty} P\left(M_{n}+\frac{1}{m} B_{X}\right) \subset \bigcap_{m=1}^{\infty} \overline{M_{n}+\frac{1}{m} B_{X}} \subset \bigcap_{m=1}^{\infty}\left(M_{n}+\frac{2}{m} B_{X}\right)=M_{n}
$$

for every $n \in \mathbb{N} \cup\{0\}$, and in particular, $\|P\|=1$.

Fix any $\gamma \in \Gamma$. We shall prove that $P \gamma \in\{\gamma, 0\}$. If $\gamma \in P X$, then, trivially, $P \gamma=\gamma$. Second, assume that $\gamma \notin P X(=\bar{A})$. Then $\gamma \notin \Phi(B)$, which implies that $\langle\gamma, b\rangle=0$ for every $b \in B$, that is, that $\gamma \in B_{\perp}$. But $B_{\perp}=P^{-1}(0)$. Hence $P \gamma=0$. Fix $\delta \in \Delta$. Assume first that $\delta \in P^{*} X^{*}$. Then $P^{*} \delta=\delta$. Otherwise, $\delta \notin P^{*} X^{*}\left(=\bar{B}^{w^{*}}\right)$. In particular, $\delta \notin \Psi(A)$. It follows that $\langle a, \delta\rangle=0$ for every $a \in A$, i.e., $\delta \in A^{\perp}$. Since $A \subset P X$ we have $\langle a, \delta\rangle=\langle P a, \delta\rangle=\left\langle a, P^{*} \delta\right\rangle$ for every $a \in A$. Then $P^{*} \delta \in P^{*} X^{*} \cap A^{\perp}=\bar{B}^{w^{*}} \cap A^{\perp}=\{0\}$ and we get $P^{*} \delta=0$. Now, once knowing how to construct one projection $P: X \rightarrow X$, the construction of the whole PRI is standard, see, e.g., [F, Section 6.1].

The next lemma is almost obvious. We include here its proof for the sake of completeness.

Lemma 31 Let $(X,\|\cdot\|)$ be a Banach space with a full $P G$. Then, every complemented subspace has also a full $P G$.

Proof. Let $Y$ be a complemented subspace of $X$, and let $\left(X^{*}, \Phi\right)$ be a PG on $X$. Let $P: X \rightarrow Y$ be a continuous linear projection. Put $\widehat{\Phi}\left(y^{*}\right):=P\left(\Phi\left(P^{*} y^{*}\right)\right)$ for $y^{*} \in Y^{*}$. We shall prove that $\left(Y^{*}, \widehat{\Phi}\right)$ is a PG for $Y$. Let $W \subset Y^{*}$ be such that $\operatorname{span}_{\mathbb{Q}}(W)=W$. Let $y^{*} \in \bar{B}_{W} w^{*} \cap[\widehat{\Phi}(W)]^{\perp}$. It follows that

$$
P^{*} y^{*} \in P^{*}\left({\overline{B_{W}}}^{*}\right) \subset{\overline{P^{*}\left(B_{W}\right)}}^{w^{*}} \subset \overline{\|P\| \cdot B_{P^{*}(W)}}{ }^{*} .
$$

It is easy to prove that $P^{*} y^{*} \in\left[\Phi\left(P^{*}(W)\right)\right]^{\perp}$. Indeed, for every $x \in \Phi\left(P^{*}(W)\right)$, we have $P x \in P\left(\Phi\left(P^{*}(W)\right)\right)=\widehat{\Phi}(W)$, and so

$$
\left\langle x, P^{*} y^{*}\right\rangle=\left\langle P x, y^{*}\right\rangle=0 .
$$

It follows that $\frac{1}{\|P\|} P^{*} y^{*} \in \overline{B_{P^{*}(W)}} w^{*} \cap\left[\Phi\left(P^{*}(W)\right)\right]^{\perp}(=\{0\})$, hence $P^{*} y^{*}=0$ and so $y^{*}=0$.

Proposition 32 Every Banach space $(X,\|\cdot\|)$ with a full $P G$ has an $M$-basis. Moreover, if $\left(P_{\alpha}\right)_{\omega_{0} \leq \alpha \leq \mu}$ is a PRI defined by the existing $P G$, this $M$-basis can be taken subordinated to the PRI.

Proof. We use a standard transfinite induction argument. Indeed, the classical Markushevich theorem gives an M-basis in every separable Banach space. Now assume 
that, given an uncountable cardinal number $\aleph$, the result has been proved for every Banach space with density $<\aleph$. Let $X$ be a Banach space with a full PG and such that dens $X=\aleph$. Then $X$ has a PRI $\left(P_{\alpha}\right)_{\omega_{0} \leq \alpha \leq \mu}$ constructed by using the existing PG. Every subspace $\left(P_{\alpha+1}-P_{\alpha}\right) X, \omega_{0} \leq \alpha<\mu$, has a full PG thanks to Lemma 31, so it has an M-basis by the induction hypothesis. A standard argument (see, e.g., [Fab97, Proposition 6.2.4]) concludes that $X$ itself has an M-basis. The way the M-basis is constructed "in differences" ensures that it is subordinated to the PRI.

Recall that a Banach space is called Asplund if every separable subspace has a separable dual space. The following result is well known (and it is just a particular case of a more general result, see Remark 34). Even in this restricted environment, it is a consequence of a deep theorem concerning M-bases in WCG Asplund spaces and some of the previous results. We include it here since it fits in this frame. We shall make use of it later on.

Proposition 33 Let $X$ be a WCG Asplund Banach space. Then $\operatorname{dens} X=\operatorname{dens} X^{*}$.

Proof Let $\left\{x_{i} ; x_{i}^{*}\right\}_{i \in I}$ be a shrinking M-basis in $X \times X^{*}$ (see [Fab97, Paragraph after Definition 6.2.3 and Theorem 8.3.3]). Obviously, $\left\{x_{i}^{*} ; x_{i}\right\}_{i \in I}$ is a fundamental biorthogonal system in $X^{*} \times X$. From Proposition 17 it follows that dens $X^{*}=\# I=$ dens $X$.

Remark 34 The result in Proposition 33 is true in a more general context. Indeed, the only requirement is that the space $X$ should be Asplund. A sketch of the proof follows. We thank B. Cascales for suggesting the following approach. Let $X$ be an Asplund space. Let $F: X \rightarrow 2^{\left(X^{*}, w^{*}\right)}$ be the duality mapping. By a result of Namioka and Phelps [NaPh75], a Banach space is Asplund if and only if the dual closed unit ball in its $w^{*}$-topology is fragmented by the norm. It is a result of Jayne and Rogers [JaRo84] that the duality mapping, in the case that the space is Asplund, has a firstBaire-class selector. Let us call $f$ such a selector in our case. Let $\left(f_{n}\right)$ be a sequence of $\|\cdot\|-\|\cdot\|$-continuous mappings from $X$ into $X^{*}$ that pointwise converge to $f$, i.e., $f_{n}(x) \rightarrow f(x)$ in the norm, for every $x \in X$. Let $D$ be a dense subset of $X$ such that $\# D=\operatorname{dens} X$. Obviously, $f_{n}(D)$ is $\|\cdot\|$-dense in $f_{n}(X)$ for every $n \in \mathbb{N}$. The set $\bigcup_{n \in \mathbb{N}} f_{n}(D)$ is $\|\cdot\|$-dense in $\bigcup_{n \in \mathbb{N}} f_{n}(X)$. By [JOPV93, Theorem 26], we have

$$
B_{X^{*}}=\overline{\mathrm{conv}}\|\cdot\| f(X)\left(\subset \overline{\mathrm{conv}}\|\cdot\|\left(\overline{\bigcup_{n \in \mathbb{N}} f_{n}(X)}\|\cdot\|\right)\right) .
$$

From this, it follows that $\operatorname{dens} X^{*} \leq \operatorname{dens} X\left(\leq \operatorname{dens} X^{*}\right)$.

The following corollary introduces a certain precision for PRI's constructed on Banach spaces with a full PG.

Proposition 35 Let $(X,\|\cdot\|)$ be a Banach space with a full $P G$. Then, there exist a $\operatorname{PRI}\left(P_{\alpha}\right)_{\omega_{0} \leq \alpha \leq \mu}$ such that dens $P_{\alpha} X=\# \alpha$ for every $\alpha \in\left[\omega_{0}, \mu\right)$. More precisely, if $\left\{x_{i} ; x_{i}^{*}\right\}_{i \in I}$ is an M-basis in $X \times X^{*}$, we can find a PRI $\left(P_{\alpha}\right)_{\omega_{0} \leq \alpha \leq \mu}$ on $X$ subordinated to $\left\{x_{i} ; i \in I\right\}$ such that dens $P_{\alpha} X=\#\left\{i \in I ; x_{i} \in P_{\alpha} X\right\}=\# \alpha$ for every $\alpha \in\left[\omega_{0}, \mu\right]$. 
Proof. By Proposition 32, there exist an M-basis $\left\{x_{i} ; x_{i}^{*}\right\}_{i \in I}$ in $X \times X^{*}$. We shall use the sets $I_{\alpha} \subset I$ defined in Proposition 28. In order to prove the last assertion, we shall use transfinite induction. The argument follows closely (notational devices included) the way Propositions 6.1.1 to 6.1.4 in [Fab97] are proved. To start with, put $P_{\omega_{0}}:=0$. Choose now some countable set $I_{0} \subset I$ and let $A_{0}:=\left\{x_{i} ; i \in I_{0}\right\}$ and $B_{0}:=\left\{x_{i}^{*} ; i \in I_{0}\right\} \subset X^{*}$. Then, according to the proof of [Fab97, Proposition 6.1.3], we produce two countable sets $A \subset X$ and $B \subset X^{*}$ such that $A_{0} \subset A, B_{0} \subset B$, and $\bar{A}$ and $\bar{B}^{w^{*}}$ are, respectively, the range of projections $P_{\omega_{0}+1}$ and $P_{\omega_{0}+1}^{*}$. Since $P_{\omega_{0}+1} X$ is separable, we get dens $P_{\omega_{0}+1} X=\# \omega_{0}\left(=\#\left(\omega_{0}+1\right)\right)$.

Assume now that the assertion holds for all ordinals $\beta<\alpha$, where $\alpha \in\left(\omega_{0}, \mu\right]$. Suppose first that $\alpha$ has a predecessor, $\alpha-1$. We take $A_{0}:=\left\{x_{i} ; i \in I_{\alpha-1}\right\} \cup\left\{x_{i_{0}}\right\}$, where $i_{0} \in I$ satisfies $i_{0} \notin I_{\alpha}$. Put $B_{0}:=\left\{x_{i}^{*} ; i \in I_{\alpha-1}\right\} \cup\left\{x_{i_{0}}^{*}\right\}$. We produce $A\left(\supset A_{0}\right)$ and $B\left(\supset B_{0}\right)$ such that $\# A=\# A_{0}$ and $\# B=\# B_{0}$, and $\bar{A}$ and $\bar{B}^{w^{*}}$ are, respectively, the range of projections $P_{\alpha}$ and $P_{\alpha}^{*}$. Then we have $\# \alpha=\#(\alpha-1)=$ dens $P_{\alpha-1} X \leq \operatorname{dens} P_{\alpha} X \leq \# \alpha$, so dens $P_{\alpha} X=\# \alpha$. If, otherwise, $\alpha$ is a limit ordinal, from the way $P_{\beta+1} X$ is constructed starting from $P_{\beta} X$, we get $\# I_{\alpha}>\# \beta$ for every $\beta<\alpha$, so $\left(\operatorname{dens} P_{\alpha} X=\right) \# I_{\alpha} \geq \# \alpha$. Finally, dens $P_{\alpha} X=\# \alpha$.

Definition 36 Let $(X,\|\cdot\|)$ be a Banach space. A PRI $\left(P_{\alpha}\right)_{\omega_{0} \leq \alpha \leq \mu}$ on $X$ is called shrinking if $\left(P_{\alpha}^{*}\right)_{\omega_{0} \leq \alpha \leq \mu}$ is a PRI on $X^{*}$.

We shall prove now that there is a connection between the notion of a shrinking M-basis and a shrinking PRI. It is worth to mention that a Banach space has a shrinking M-basis if and only if it is WCG and Asplund (see, e.g., [Fab97, paragraph after Definition 6.2.3 and Theorem 8.3.3]).

Proposition 37 Let $(X,\|\cdot\|)$ a Banach space with a shrinking $M$-basis $\left\{x_{i} ; x_{i}\right\}_{i \in I}$ in $X \times X^{*}$. Then, every PRI $\left(P_{\alpha}\right)_{\omega_{0} \leq \alpha \leq \mu}$ on $X$ subordinated to $\left\{x_{i} ; i \in I\right\}$ is shrinking.

Proof. The existence of such a PRI is guarantee by Proposition 30 and Remark 25, since it follows that the set $\left\{x_{i} ; i \in I\right\}$ countably supports $X^{*}$. Certainly, $\left(P_{\alpha}^{*}\right)_{\omega_{0} \leq \alpha \leq \mu}$ is a family of projections on $X^{*}$ that satisfy $P_{\omega_{0}}^{*}=0$ and $P_{\mu}^{*}=\operatorname{Id}_{X^{*}}$ and (i) to (ii) in Definition 20. From Proposition 33 we get that dens $P_{\alpha}^{*} X^{*}=\operatorname{dens} P_{\alpha} X \leq \# \alpha$ for every $\alpha \in\left[\omega_{0}, \mu\right]$. This is (iii) in the aforesaid definition. Let $x^{*} \in X^{*}$. Fix $\varepsilon>0$. There exists $i_{1}, \ldots, i_{n}$ in $I$ and $\lambda_{1}, \ldots, \lambda_{n}$ in $\mathbb{R}$ such that $l^{*}:=\sum_{k=1}^{n} \lambda_{k} x_{i_{k}}^{*}$ satisfies $\left\|x^{*}-l^{*}\right\|<\varepsilon$. From Lemma 27 and Proposition 28 we know that there exists $\alpha_{0} \in\left[\omega_{0}, \mu\right)$ such that $x_{i_{k}}^{*} \in P_{\alpha_{0}}^{*} X^{*}$ for all $k=1,2, \ldots, n$. Let $\alpha \in\left[\alpha_{0}, \mu\right)$. Then,

$$
\left\|x^{*}-P_{\alpha}^{*} x^{*}\right\| \leq\left\|x^{*}-P_{\alpha}^{*} l^{*}\right\|+\left\|P_{\alpha}^{*} l^{*}-P_{\alpha}^{*} x^{*}\right\| \leq\left\|x^{*}-l^{*}\right\|+\left\|l^{*}-x^{*}\right\|<2 \varepsilon .
$$

Since $\varepsilon>0$ was arbitrary, it follows that $P_{\alpha}^{*} x^{*} \rightarrow x^{*}$ in norm. This is (iv) in Definition 20 . 
Remark 38 The converse to Proposition 37 does not hold. Indeed, let $X$ be a nonreflexive WCG Asplund space. There exists a non-reflexive separable subspace $Y$ (that is also Asplund). By a result of Zippin [Zi68] there exists a non-shrinking (Schauder) basis in $Y \times Y^{*}$. This Schauder basis can be extended to an M-basis $\left\{x_{i} ; x_{i}^{*}\right\}_{i \in I}$ in $X \times X^{*}$ (see Corollary 57). This M-basis cannot be shrinking, due to Proposition 19 . Let $\left\{e_{\gamma} ; e_{\gamma}^{*}\right\}_{\gamma \in \Gamma}$ be a shrinking M-basis in $X \times X^{*}$. The set $\left\{x_{i} ; i \in I\right\} \cup\left\{e_{\gamma} ; \gamma \in \Gamma\right\}$ countably supports $X^{*}$. Then we can find a PRI $\left(P_{\alpha}\right)_{\omega_{0} \leq \alpha \leq \mu}$ on $X$ subordinated to $\left\{x_{i} ; i \in I\right\} \cup\left\{e_{\gamma} ; \gamma \in \Gamma\right\}$, hence, by Proposition 37, the PRI is shrinking and subordinated to $\left\{x_{i} ; i \in I\right\}$, a non-shrinking M-basis in $X \times X^{*}$.

The following concept can be found in [Fab97, Definition 6.2.6]. Let us recall it here for the sake of completeness.

Definition 39 Let $X$ be a nonseparable Banach space $X, \mu$ be the first ordinal with card $\mu=$ dens $X$. A separable projectional resolution of the identity (SPRI, in short) on $X$ is a "long sequence" $\left(P_{\alpha} ; \omega_{0} \leq \alpha \leq \mu\right)$ of linear projections on $X$ such that $P_{\omega_{0}} \equiv 0, P_{\mu}$ is the identity mapping, and the following hold:

(i) $\left\|P_{\alpha}\right\|<+\infty$ if $\omega_{0} \leq \alpha \leq \mu$,

(ii) $\left(P_{\alpha+1}-P_{\alpha}\right) X$ is separable if $\omega_{0} \leq \alpha<\mu$,

(iii) $P_{\alpha} P_{\beta}=P_{\beta} P_{\alpha}=P_{\beta}$ if $\omega_{0} \leq \beta \leq \alpha \leq \mu$, and

(iv) $x \in \overline{\operatorname{sp}}\left\{\left(P_{\alpha+1}-P_{\alpha}\right) x ; \omega_{0} \leq \alpha<\mu\right\}$ for all $x \in X$.

The following simple result is similar to Lemma 27.

Lemma 40 Let $X$ be a Banach space and $\left(P_{\alpha}\right)_{\omega_{0} \leq \alpha \leq \mu}$ a SPRI on $X$. Let $\Gamma \subset X$ and $\Delta \subset X^{*}$ be two sets, the first one subordinated to $\left(P_{\alpha}\right)_{\omega_{0} \leq \alpha \leq \mu}$, the second one to $\left(P_{\alpha}^{*}\right)_{\omega_{0} \leq \alpha \leq \mu}$. Then $\Gamma \subset \bigcup_{\omega_{0} \leq \alpha<\mu} P_{\alpha} X$ and $\Delta \subset \bigcup_{\omega_{0} \leq \alpha<\mu} P_{\alpha}^{*} X^{*}$.

Proof. Fix $x \in \Gamma$. Assume that for no $\alpha \in\left[\omega_{0}, \mu\right)$ we have $x \in P_{\alpha} X$. Then, since $\Gamma$ is subordinated to $\left(P_{\alpha}\right)_{\omega_{0} \leq \alpha \leq \mu}$ we have $P_{\alpha} x=0$ for every $\alpha \in\left[\omega_{0}, \mu\right)$. But this implies, from (iv) in Definition 39, that $x=0$, contradicting the assumption.

Fix any $x^{*} \in X^{*}$. We shall prove first that $x^{*} \in \overline{\operatorname{span}} w^{*}\left\{P_{\alpha}^{*} X^{*} ; \omega_{0} \leq \alpha<\mu\right\}$. Assume not. Then we can find $x \in X$ such that $\left(\left\langle P_{\alpha} x, x^{*}\right\rangle=\right)\left\langle x, P_{\alpha}^{*} x^{*}\right\rangle=0$ for every $\alpha \in\left[\omega_{0}, \mu\right)$ and $\left\langle x, x^{*}\right\rangle=1$. In particular, $\left\langle\left(P_{\alpha+1}-P_{\alpha}\right) x, x^{*}\right\rangle=0$ for every $\alpha \in\left[\omega_{0}, \mu\right)$. It follows from (iv) in Definition 39 that $\left\langle x, x^{*}\right\rangle=0$, a contradiction.

Now let $\delta \in \Delta$. Assume that for no $\alpha \in\left[\omega_{0}, \mu\right)$ we have $\delta \in P_{\alpha}^{*} X^{*}$. Then, due to the subordination property, $P_{\alpha}^{*} \delta=0$ for all $\alpha \in\left[\omega_{0}, \mu\right)$. By the previous paragraph, we get $\delta=0$, in contradiction with the assumption.

We shall need the following result, a consequence of Proposition 30.

Proposition 41 Let $(X,\|\cdot\|)$ be a Banach space with a full $P G$. Let $\Gamma \subset X$ and $\Delta \subset X^{*}$ be sets such that $\Gamma$ countably supports $X^{*}$ and $\Delta$ countably supports $X$. Then there exists on $X$ a SPRI subordinated to $\Gamma$ and such that $\Delta$ is subordinated to the family of adjoint projections. 
Proof. We shall prove the statement by transfinite induction on the density character of $X$. If $X$ is separable there is nothing to prove. Assume now that the proposition holds for all Banach spaces $X$ with dens $X<\aleph$, where $\aleph$ a given uncountable cardinal. Let $X$ be a Banach space as in the statement, with dens $X=\aleph$. By Proposition 30 we can find a PRI $\left(P_{\alpha}\right)_{\omega_{0} \leq \alpha \leq \mu}$ on $X$ subordinated to $\Gamma$, and such that $\left(P_{\alpha}^{*}\right)_{\omega_{0} \leq \alpha \leq \mu}$ is subordinated to $\Delta$. Fix $\omega_{0} \leq \alpha<\mu$. Put $Q_{\alpha}:=P_{\alpha+1}-P_{\alpha}$. Then $Q_{\alpha} X$ is a Banach space with density less that $\aleph$. Moreover, $Q_{\alpha} \Gamma$ countably supports $Q_{\alpha}^{*} X^{*}$ and $Q_{\alpha}^{*} \Delta$ countably supports $Q_{\alpha} X$. Indeed, fix $x^{*} \in X^{*}$. Then

$$
\#\left\{Q_{\alpha} \gamma ;\left\langle\gamma, Q_{\alpha}^{*} x^{*}\right\rangle \neq 0\right\} \leq \#\left\{\gamma \in \Gamma ;\left\langle\gamma, x^{*}\right\rangle \neq 0\right\} \cup\{0\}\left(\leq \omega_{0}\right) .
$$

The argument for $Q_{\alpha}^{*} \Delta$ is similar.

The space $Q_{\alpha} X$ is either separable or by the induction hypothesis we can find a SPRI on $Q_{\alpha} X$ subordinated to $\Gamma \cap Q_{\alpha} X$ and such that the family of adjoint projections is subordinated to $\Delta \cap Q_{\alpha}^{*} X^{*}$. In order to construct a SPRI on $X$, use the technique presented in [Fab97, Proposition 6.2.7]. The SPRI thus constructed has the sought properties.

The following simple lemma is similar to Propositions 28 and 29, now in the case of a separable PRI.

Lemma 42 Let $X$ be a Banach space with an $\operatorname{SPRI}\left(P_{\alpha}\right)_{\omega_{0} \leq \alpha \leq \mu}$ and let $\left\{x_{i} ; x_{i}^{*}\right\}_{i \in I}$ be an $M$-basis on $X \times X^{*}$ such that $\left\{x_{i} ; i \in I\right\}$ is subordinated to $\left(P_{\alpha}\right)_{\omega_{0} \leq \alpha \leq \mu}$. For every $\alpha \in\left[\omega_{0}, \mu\right)$ put $Q_{\alpha}:=P_{\alpha+1}-P_{\alpha}$. Then we have

(i) $\left\{x_{i}\right\}_{i \in I}$ is subordinated to $\left(Q_{\alpha}\right)_{\omega_{0} \leq \alpha<\mu}$.

(ii) For $\omega_{0} \leq \alpha<\mu$ put $D_{\alpha}:=\left\{i \in I ; Q_{\alpha} x_{i}=x_{i}\right\}$. Then $\bigcup_{\omega_{0} \leq \alpha<\mu} D_{\alpha}=I$.

(iii) For $\omega_{0} \leq \alpha<\mu, D_{\alpha}=\left\{i \in I ; Q_{\alpha}^{*} x_{i}^{*}=x_{i}^{*}\right\}$.

(iv) $\left\{x_{i}^{*}\right\}_{i \in I}$ is subordinated to $\left(Q_{\alpha}^{*}\right)_{\omega_{0} \leq \alpha<\mu}$.

(v) For $\omega_{0} \leq \alpha<\mu,\left\{x_{i} ; x_{i}^{*}\right\}_{i \in D_{\alpha}}$ is an M-basis in $Q_{\alpha} X \times\left(Q_{\alpha} X\right)^{*}$ (and, in particular, $\left.\# D_{\alpha} \leq \aleph_{0}\right)$.

Proof. It follows a pattern similar to the proof of Propositions 28 and 29. We provide here only a sketch. (i) should be clear. (ii) follows from (iv) in Definition 39: indeed, if $i \in I \backslash \bigcup_{\omega_{0} \leq \alpha<\mu} D_{\alpha}$ it follows from (i) here that $Q_{\alpha} x_{i}=0$ for all $\omega_{0} \leq \alpha<\mu$ and so $x_{i}=0$, a contradiction. To prove (iii), (iv) and (v) we follow arguments already used in the proof of Propositions 28 and 29. The last assertion is a consequence of the separability of $Q_{\alpha} X$, (iv) and Proposition 17.

\subsection{Examples}

\subsubsection{WCG Banach spaces}

A Banach space is weakly compactly generated (WCG, in short) if it contains a linearly dense weakly compact subset. The following result is less general than Theorem 50, to 
be proved later. However, it gives in a very natural way a full projectional generator $\left(X^{*}, \Phi\right)$ in a WCG Banach space $X$. This is the reason why we prefer to present it here. Moreover, it gives the opportunity to rediscover the Amir-Lindenstrauss classical result on the existence of a PRI on any WCG Banach space, [AmLi68] (see also [FHHMPZ01, Theorem 11.6]). The fact that, moreover, the range of the mapping $\Phi$ is contained in a weakly compact set generating $X$ gives the well-known result (again due to Amir and Lindenstrauss, [AmLi68], see [FHHMPZ01, Theorem 11.12]) that $X$ contains a weakly compact Markushevich basis, i.e., a Markushevich basis $\left\{x_{\gamma} ; x_{\gamma}^{*}\right\}_{\gamma \in \Gamma}$ such that $\left\{x_{\gamma} ; \gamma \in \Gamma\right\} \cup\{0\}$ is a weakly compact set (see Corollary 44). We include the statement and the proof of Proposition 43 here because it is a simple exercise by using the Theorem of Mackey-Arens. In fact, [HMVZ07, Proposition 3.43] uses our approach.

Proposition 43 Let $X$ be a WCG Banach space generated by an absolutely convex and weakly compact set $K$. Then, $X$ has a full (single-valued) projectional generator $\left(X^{*}, \Phi\right)$ such that $\Phi\left(x^{*}\right) \in K$ for all $x^{*} \in X^{*}$

Proof: Given $x^{*} \in X^{*}$, let $\Phi\left(x^{*}\right)$ be an element in $K$ such that $\left\langle\Phi\left(x^{*}\right), x^{*}\right\rangle=$ $\sup \left|\left\langle K, x^{*}\right\rangle\right|$. We Claim that $\left(X^{*}, \Phi\right)$ is a PG. In order to prove this, let $W \subset X^{*}$ be such that $\operatorname{span}_{\mathbb{Q}}(W)=W$. Let $x^{*} \in \Phi(W)^{\perp} \cap \bar{B}_{W}^{w^{*}}$. By the Mackey-Arens theorem, we have $\overline{B_{W}} w^{*} \subset{\overline{B_{W}}}^{\mathcal{T}_{K}}$, where $\mathcal{T}_{K}$ is the topology on $X^{*}$ of the uniform convergence on $K$. Consider any $\varepsilon>0$. Find then $y^{*} \in B_{W}$ such that $\sup \left|\left\langle K, x^{*}-y^{*}\right\rangle\right|<\varepsilon$. Then

$$
\begin{gathered}
\sup \left\langle K, x^{*}\right\rangle \leq \sup \left\langle K, x^{*}-y^{*}\right\rangle+\sup \left\langle K, y^{*}\right\rangle<\varepsilon+\sup \left\langle K, y^{*}\right\rangle \\
=\varepsilon+\left\langle\Phi\left(y^{*}\right), y^{*}\right\rangle=\varepsilon+\left\langle\Phi\left(y^{*}\right), y^{*}-x^{*}\right\rangle \leq \varepsilon+\sup \left\langle K, y^{*}-x^{*}\right\rangle<2 \varepsilon .
\end{gathered}
$$

As $\varepsilon>0$ was arbitrary, we get $\left.x^{*}\right|_{K} \equiv 0$, and so $x^{*}=0$. This proves the Claim and the result.

Corollary 44 (Amir-Lindenstrauss) A Banach space is WCG if and only if it has a weakly compact Markushevich basis.

Proof. Let $K \subset X$ be an absolutely convex and weakly compact subset of $X$ that generates $X$. It exists because of the Krein-Milman Theorem. Proposition 43 ensures the existence of a full projectional generator for $X$. It will be enough now to apply Proposition 30 to the case of the sequence $\left(M_{n}\right)$, where $M_{n}:=K$ for all $n \in \mathbb{N}$, to conclude that there exists a PRI $\left(P_{\alpha}\right)_{\omega_{0} \leq \alpha \leq \mu}$ on $X$ such that $P_{\alpha} K \subset K$ for every $\omega_{0} \leq \alpha \leq \mu$. The construction of a Markushevich basis $\left\{x_{\gamma} ; x_{\gamma}^{*}\right\}_{\gamma \in \Gamma}$ in $X \times X^{*}$ such that $\left\{x_{\gamma} ; \gamma \in \Gamma\right\} \subset K$ follows from a simple transfinite induction process. Indeed, in a separable subspace $P_{\alpha} X$ of $X$ and given a generating countable subset $S \subset P_{\alpha} K$, the classical Markushevich construction gives a countable Markushevich basis $\left\{x_{n} ; x_{n}^{*}\right\}_{n \in \mathbb{N}}$ such that $\operatorname{span}\left\{x_{n} ; n \in \mathbb{N}\right\}=\operatorname{span} S$. By homogeneity, we may assume that $\left\{x_{n} ; n \in \mathbb{N}\right\} \subset K$. Now, the inductive argument provides a Markushevich basis in each piece $\left(P_{\alpha+1}-P_{\alpha}\right) X$ lying in $\left(P_{\alpha+1}-P_{\alpha}\right) K \subset 2 K$, and so, again by 
homogeneity, in $K$. It is enough now to glue all these bases together as in [Fab97, Proposition 6.2.4].

Remark 45 As a consequence of Proposition 66 below, the weakly compact M-basis that exists in every WCG Banach space due to Corollary 44 has the (AL)-property (see Definition 63).

Remark 46 A description of a PG for a Banach space generated by a RadonNikodym compactum in the weak or the weak* topology is provided in [CNO03]. This class of Banach spaces includes the WCG and the dual spaces of Asplund spaces. This is an observation of J. Orihuela.

\subsubsection{WCD Banach spaces}

Let $X$ be a Banach space, and let $S$ be a non-empty subset of $X$. We denote, as before, by $\operatorname{span}_{\mathbb{Q}}(S)$, the set of all rational linear combinations of elements in $S$, i.e., $\operatorname{span}_{\mathbb{Q}}(S):=\left\{\sum_{i=1}^{n} q_{i} s_{i} ; q_{i} \in \mathbb{Q}, s_{i} \in S, i=1,2, \ldots, n, n \in \mathbb{N}\right\}$.

WCD (also called Vašák) Banach spaces were introduced in Definition 2. Every WCG Banach space is WCD; it is enough to consider the sequence $\left(K_{n}\right)$ of subsets of $X^{* *}$ given by $K_{n}:=\overline{n K+(1 / n) B_{X}} w^{*}, n \in \mathbb{N}$, in Definition 2. In [Fab97, Proposition 7.2.1] it is proved that every WCD Banach space has a projectional generator. The proof uses a characterization of WCD Banach spaces in terms of usco mappings. We provide another description of a (full) projectional generator for a WCD Banach space, following the approach in [DGZ, Section VI.2]. There, the definition of the projectional generator is somehow hidden in the construction. Here we explicit the description and check that, indeed, it is a projectional generator in the sense introduced in Definition 21. This construction should also be compared to the way in which a projectional generator is constructed in [Fab97, Section 8.2] to ensure that the dual space of an Asplund Banach space has indeed one. Another description of a projectional generator for a WCD Banach space is given in [OV89]. This is a remark of one of the referees.

In this section $X$ denotes a Vašák space and $\left(K_{n}\right)$ is the sequence of subsets of $X^{* *}$ introduced in Definition 2.

Let $\mathcal{S}$ be the (countable) family of all finite subsets of $\mathbb{N}$. Put

$$
L_{s}:={\overline{X \cap \bigcap_{n \in s} K_{n}}}^{w^{*}}, s \in \mathcal{S} .
$$

For $x^{*} \in X^{*}$, let $\Phi\left(x^{*}\right)$ be an at most countable subset of $X$ such that $\sup _{x \in L_{s}}\left|\left\langle x, x^{*}\right\rangle\right|=$ $\sup _{x \in L_{s} \cap \Phi\left(x^{*}\right)}\left|\left\langle x, x^{*}\right\rangle\right|$ for every $s \in \mathcal{S}$. This defines a set-valued mapping from $X^{*}$ into the family of countable subsets of $X$. 
Proposition 47 The mapping $\Phi$ defined above is a (full) projectional generator for $X$.

Proof. By the very definition, $\Phi\left(x^{*}\right)$ is at most countable for every $x^{*} \in X^{*}$. Let us check $\Phi$ also satisfies the property from Definition 21. Take $B \subset X^{*}$ such that $\operatorname{span}_{\mathbb{Q}}(B)=B$ and assume that for some $x^{*} \in \Phi(B)^{\perp} \cap \bar{B}^{w^{*}}$ we have $x^{*} \neq 0$. Find $x \in X$ so that $\left\langle x, x^{*}\right\rangle=1$. Let $\left\{n \in \mathbb{N} ; x \in K_{n}\right\}=\left\{n_{k} ; k \in \mathbb{N}\right\}$. Put $K:=\bigcap_{i=1}^{\infty} L_{n_{1} n_{2} \ldots n_{i}}$. Observe that $L_{n_{1} n_{2} \ldots n_{i}} \subset L_{n_{1}}$ for all $i \in \mathbb{N}$, and that $L_{n_{1}}$ is bounded, Let $M:=\sup \left\{\left\|x^{* *}\right\| ; x^{* *} \in L_{n_{1}}\right\}$. Certainly, $x \in K$, and $K$ is a weakly compact subset of $X$. Obviously, $\bar{B}^{w^{*}}={\overline{\left(\bar{B}^{\|\cdot\|}\right.}}^{w^{*}}$, and the set $\bar{B}^{\|\cdot\|}$ is a (closed) linear subspace. The theorem of Mackey-Arens (see, e.g., [Ko69, Theorem §21.4.2]) ensures that $\bar{B}^{w^{*}}={\overline{\left(\bar{B}^{\|\cdot\|}\right)}}^{\mu^{*}}$, where $\mu^{*}$ denotes the (Mackey) topology on $X^{*}$ of the uniform convergence on the family of all absolutely convex and $w$-compact subsets of $X$. So we can find $y^{*} \in \bar{B}^{\|\cdot\|}$ such that $\sup \left|\left\langle K, x^{*}-y^{*}\right\rangle\right|<1 / 8$. Find now $y_{0}^{*} \in B$ such that $\left\|y^{*}-y_{0}^{*}\right\|<1 /(8 M)$.

The set $U:=\left\{x^{* *} \in X^{* *} ;\left|\left\langle x^{* *}, y^{*}-x^{*}\right\rangle\right|<1 / 8\right\}$ is a $w^{*}$-open subset of $X^{* *}$ containing $K$, so there exists $k \in \mathbb{N}$ such that $(K \subset) L_{n_{1}, \ldots, n_{k}} \subset U$. Notice that $\left|\left\langle x, y^{*}\right\rangle-1\right|=\left|\left\langle x, y^{*}-x^{*}\right\rangle\right|<1 / 8$, hence $\left|\left\langle x, y^{*}\right\rangle\right|>7 / 8$, and

$$
\left|\left\langle x, y_{0}^{*}\right\rangle\right|=\left|\left\langle x, y_{0}^{*}-y^{*}+y^{*}\right\rangle\right|>\frac{7}{8}-\frac{M}{8 M}=\frac{6}{8} .
$$

Therefore

$$
\sup \left\langle L_{n_{1} \ldots n_{k}} \cap \Phi\left(y_{0}^{*}\right), y_{0}^{*}\right\rangle=\sup \left\langle L_{n_{1} \ldots n_{k}}, y_{0}^{*}\right\rangle \geq\left\langle x, y_{0}^{*}\right\rangle>\frac{6}{8} .
$$

and this gives

$$
\sup \left\langle L_{n_{1} \ldots n_{k}} \cap \Phi\left(y_{0}^{*}\right), y^{*}\right\rangle>\frac{5}{8} .
$$

Since $L_{n_{1} \ldots n_{k}} \subset U$, we get

$$
\sup \left\langle L_{n_{1} \ldots n_{k}} \cap \Phi\left(y_{0}^{*}\right), x^{*}\right\rangle>\frac{4}{8}=\frac{1}{2} .
$$

This is a contradiction, since $x^{*} \in(\Phi(B))^{\perp}$, and $y_{0}^{*} \in B$.

As it was mentioned immediately after Definition 21, the existence of a projectional resolution of the identity follows from the existence of a projectional generator. So, as a consequence of Proposition 47, we get the well-known result below (see, e.g., [DGZ, Theorem VI.2.5]).

Corollary 48 Every WCD Banach space has a projectional resolution of the identity. 


\subsubsection{WLD Banach spaces}

Definition 49 A Banach space is called weakly Lindelöf determined (in short, WLD) if the dual unit ball of it, equipped with the $w^{*}$-topology, is Corson, i.e., it is a compact subspace of the topological space $\Sigma(\Gamma)$ consisting of all elements in $\mathbb{R}^{\Gamma}$ having only countably many non-zero coordinates. Here, $\mathbb{R}^{\Gamma}$ is endowed with the product (i.e., pointwise) topology $\mathcal{T}_{p}$.

Every WCD Banach space is WLD (Corollary 54). In this section, we give a characterization of WLD Banach spaces in terms of the existence of a certain projectional generator. It enlarges the current information about such class of non-separable Banach spaces.

The following result characterizes Banach spaces having a full PG. It is known that a WLD Banach space has such a PG (see, for example, [Fab97, Proposition 8.3.1]). That a WLD is characterized by the existence of an M-basis (or just a linearly dense subset) that countably supports $X^{*}$ is also known (see, for example, [FGMZ04] or [FHHMPZ01, Theorem 12.50]).

Theorem 50 Let $X$ be a Banach space. Then the following assertions are equivalent.

(i) $X$ is WLD.

(ii) $X$ has a full $P G$.

(iii) $X$ has an $M$-basis $\left\{x_{\gamma} ; x_{\gamma}^{*}\right\}_{\gamma \in \Gamma}$ such that $\left\{x_{\gamma} ; \gamma \in \Gamma\right\}$ countably supports $X^{*}$.

(iv) $X$ contains a linearly dense subset $G$ that countably supports $X^{*}$.

Actually, if one of the above conditions holds, then every $M$-basis $\left\{x_{\gamma} ; x_{\gamma}^{*}\right\}_{\gamma \in \Gamma}$ in $X \times X^{*}$ has the property that $\left\{x_{\gamma} ; \gamma \in \Gamma\right\}$ countably supports $X^{*}$.

Proof. (i) $\Rightarrow$ (ii) is proved, for example, in [Fab97, Proposition 8.3.1]. We provide here a (somehow) streamlined proof. $\left(B_{X^{*}}, w^{*}\right)$ is a Corson compactum; hence, for some non-empty $\Gamma$, it is a subspace of $\left(\Sigma(\Gamma), \mathcal{T}_{p}\right)$. Given $\gamma \in \Gamma$, let $\pi_{\gamma}: \Sigma(\Gamma) \rightarrow \mathbb{R}$ be the $\gamma$-th coordinate mapping; its restriction to $B_{X^{*}}$, denoted again $\pi_{\gamma}$, is an element in $C\left(\left(B_{X^{*}}, w^{*}\right)\right)$. In this last space, the algebra generated by the elements in $X$ and the constant functions is, by Stone-Weierstrass theorem, norm-dense, so there exists a countable set $X_{\gamma} \subset X$ such that $\pi_{\gamma}$ is in the norm-closure of the algebra $\mathcal{A}\left(X_{\gamma}, 1\right)$ generated by $X_{\gamma}$ and the constant function 1 equal to 1 everywhere on $\left(B_{X^{*}}, w^{*}\right)$. Define $\Phi: X^{*} \rightarrow 2^{X}$ as

$$
\Phi\left(x^{*}\right)= \begin{cases}\{0\}, & \text { if } x^{*} \in\left(X^{*} \backslash B_{X^{*}}\right) \cup\{0\}, \\ \bigcup_{\pi_{\gamma}\left(x^{*}\right) \neq \pi_{\gamma}(0)} X_{\gamma}, & \text { if } x^{*} \in B_{X^{*}} \backslash\{0\} .\end{cases}
$$

We Claim that $\left(X^{*}, \Phi\right)$ is a PG. To prove the Claim, take $W \subset X^{*}$ such that $\operatorname{span}_{\mathbb{Q}} W=W$. Let $x^{*} \in \Phi(W)^{\perp} \cap \overline{B_{W}} w^{*}$. Assume $x^{*} \neq 0$. Then there exists $\gamma \in \Gamma$ such that $\pi_{\gamma}\left(x^{*}\right) \neq \pi_{\gamma}(0)$. As $x^{*} \in \overline{B_{W}} w^{*}$, there exists $w^{*} \in B_{W}$ such that $\pi_{\gamma}\left(w^{*}\right) \neq \pi_{\gamma}(0)$. Then $X_{\gamma} \subset \Phi\left(w^{*}\right)$. Since $x^{*} \in \Phi(W)^{\perp}$ and $\Phi\left(w^{*}\right) \subset \Phi(W)$, we have $\left\langle X_{\gamma}, x^{*}\right\rangle=0$. Now, every element of $\mathcal{A}\left(X_{\gamma}, 1\right)$ is of the form $f:=a_{0}+$ 
$\sum_{i=1}^{k} a_{i} \prod_{j=1}^{n_{i}} x_{i, j}$, where $a_{0}, a_{i}$ are constant functions and $x_{i, j} \in X_{\gamma}$. It follows that $f\left(x^{*}\right)=a_{0}=f(0)$. Then, since $\pi_{\gamma}$ is in the norm-closure of $\mathcal{A}\left(X_{\gamma}, 1\right)$ we get $\pi_{\gamma}\left(x^{*}\right)=\pi_{\gamma}(0)$, a contradiction.

(ii) $\Rightarrow$ (iii) We proved in Proposition 32 that every Banach space with a full PG has an M-basis. We shall prove now that every M-basis $\left\{x_{\gamma} ; x_{\gamma}^{*}\right\}_{\gamma \in \Gamma}$ in $X \times X^{*}$ satisfies that $\left\{x_{\gamma} ; \gamma \in \Gamma\right\}$ countably supports $X^{*}$. Thus the last statement of our theorem will also be proved. Given $x^{*} \in X^{*}$, put supp $\left(x^{*}\right):=\left\{\gamma \in \Gamma ;\left\langle x_{\gamma}, x^{*}\right\rangle \neq 0\right\}$.

Let $S:=\left\{x^{*} \in X^{*}\right.$; \#supp $\left.\left(x^{*}\right) \leq \aleph_{0}\right\}$; it is a linear subspace of $X^{*}$. Since $S$ contains all $x_{\gamma}^{*}$ 's, it is $w^{*}$-dense. We shall prove that $S \cap B_{X^{*}}$ is $w^{*}$-closed. Then the BanachDieudonné Theorem will yield that $S$ is $w^{*}$-closed, hence $S=X^{*}$ and so $\left\{x_{\gamma} ; \gamma \in \Gamma\right\}$

will countably support $X^{*}$, as we wish to prove. So, pick any $x_{0}^{*} \in \overline{S \cap B_{X^{*}}} w^{*}$.

Put $W_{1}=\operatorname{sp}_{\mathbb{Q}}\left\{x_{0}^{*}\right\}$; this is a countable set. $\Phi\left(W_{1}\right)$ is also a countable set. Let us enumerate it as $\Phi\left(W_{1}\right)=\left\{x_{1}^{1}, x_{2}^{1}, \ldots\right\}$. Find $x_{1}^{*} \in S \cap B_{X^{*}}$ so that $\mid\left\langle x_{1}^{1}, x_{1}^{*}-\right.$ $\left.x_{0}^{*}\right\rangle \mid<1$. Put $W_{2}=\operatorname{sp}_{\mathbb{Q}}\left\{x_{0}^{*}, x_{1}^{*}\right\}$. The set $\Phi\left(W_{2}\right)$ is again countable. Write then $\Phi\left(W_{2}\right)=\left\{x_{1}^{2}, x_{2}^{2}, \ldots\right\}$. Find $x_{2}^{*} \in S \cap B_{X^{*}}$ so that $\left|\left\langle x_{j}^{i}, x_{2}^{*}-x_{0}^{*}\right\rangle\right|<\frac{1}{2}$ for each $i, j \in\{1,2\}$. Put $W_{3}=\operatorname{sp}_{\mathbb{Q}}\left\{x_{0}^{*}, x_{1}^{*}, x_{2}^{*}\right\}$. The set $\Phi\left(W_{3}\right)$ is again countable. Write then $\Phi\left(W_{3}\right)=\left\{x_{1}^{3}, x_{2}^{3}, \ldots\right\}$. Find $x_{3}^{*} \in S \cap B_{X^{*}}$ so that $\left|\left\langle x_{j}^{i}, x_{3}^{*}-x_{0}^{*}\right\rangle\right|<\frac{1}{3}$ for each $i, j \in\{1,2,3\}$. Continuing in a obvious way, we get a sequence $\left(x_{n}^{*}\right)_{n=1}^{\infty}$ in $S \cap B_{X^{*}}$, "rationally" linear countable sets $W_{1} \subset W_{2} \subset \cdots \subset X^{*}$, and vectors $x_{j}^{i}, i, j \in \mathbb{N}$. Put $W:=W_{1} \cup W_{2} \cup \cdots$; then $\operatorname{sp}_{\mathbb{Q}} W=W$. Let $y^{*} \in B_{X^{*}}$ be a $w^{*}$-cluster point of the sequence $\left(x_{n}^{*}\right)$. Pick any $x \in \Phi(W)$. Then $x=x_{j}^{i}$ for suitable $i, j \in \mathbb{N}$. Then for $n \in \mathbb{N}$, with $n>\max \{i, j\}$, we have $\left|\left\langle x, x_{n}^{*}-x_{0}^{*}\right\rangle\right|=\left|\left\langle x_{j}^{i}, x_{n}^{*}-x_{0}^{*}\right\rangle\right|<\frac{1}{n}$, and hence $\left\langle x, y^{*}-x_{0}^{*}\right\rangle=0$. We thus showed that $y^{*}-x_{0}^{*} \in \Phi(W)^{\perp}$. On the other hand $\frac{1}{2}\left(y^{*}-x_{0}^{*}\right) \in \overline{B_{W}} w^{*}$. Therefore $y^{*}-x_{0}^{*}=0$. And, since $y^{*}$, being a $w^{*}$-cluster point of the sequence $\left(x_{n}^{*}\right)$, has at most a countable support, we can conclude that $x_{0}^{*}=y^{*} \in S \cap B_{X^{*}}$.

(iii) $\Rightarrow$ (iv) is trivial.

(iv) $\Rightarrow\left(\right.$ i) holds since the mapping $x^{*} \mapsto\left(\left\langle\gamma, x^{*}\right\rangle\right)_{\gamma \in \Gamma}$ from $X^{*}$ into $\mathbb{R}^{\Gamma}$ shows that $\left(B_{X^{*}}, w^{*}\right)$ is a Corson compactum.

Remark 51 Following a suggestion of J. Orihuela, we provide here a different approach to the implication (ii) $\Rightarrow$ (i) in Theorem 50. Assume that $X$ is a Banach space with a full PG. We proved in Proposition 32 that $X$ has then an M-basis. Theorem 5.1 in [CNO03] proves that $\left(X, \gamma\left(X, X^{*}\right)\right)$ is Lindelöf, where $\gamma\left(X, X^{*}\right)$ is the topology on $X$ of the uniform convergence on all bounded sequences in $X^{*}$. In particular, since $w\left(X, X^{*}\right)$ is coarser than $\gamma\left(X, X^{*}\right)$, it follows that $\left(X, w\left(X, X^{*}\right)\right)$ is Lindelöf. It is enough to use now the fact that, for spaces $X$ with an M-basis, to be $w\left(X, X^{*}\right)$ Lindelöf and to be WLD are equivalent [O92], [VWZ94], (see, e.g., [FHHMPZ01, Theorem 12.48]). The information given in [CNO03, Theorem 6.1] is also relevant in this context: A Banach space $X$ is $W L D$ if and only if it has a PG and property $(C)$ of Corson (a weaker property than to be $w\left(X, X^{*}\right)$-Lindelöf). 
It is also worth to recall that a description of a PG for WLD Banach spaces was given in [O92].

Remark 52 Lemma 31 and Proposition 32 give that every complemented subspace of a WLD Banach space has an M-basis. This holds, too, for an arbitrary (closed) subspace $Y$ of a WLD Banach space $X$. The proof uses again a transfinite induction argument on the density of $Y$. Assume first that $Y$ is separable. Then the result follows from the classical Markushevich theorem. Let $\aleph$ be an uncountable cardinal number. Assume that the result holds for every Banach space of density $<\aleph$ which is a subspace of a Banach space with a full PG. Let $Y$ be a subspace of density $\aleph$ of $X$. Since $X$ has a $\mathrm{PG}$, there exists a complemented subspace $Z$ of $X$ such that $Y \subset Z \subset X$ and dens $Z=\operatorname{dens} Y$. Let $\mu$ be the first ordinal with cardinal dens $Z$. Lemma 31 ensures that $Z$ has also a full PG, so there exists a PRI $\left(P_{\alpha}\right)_{\omega_{0} \leq \alpha \leq \mu}$ on $Z$ such that every $P_{\alpha}$ fixes $Y$ (see Proposition 30), and then the long sequence of their restrictions to $Y$ provides a PRI on $Y$. By the induction hypothesis, every $\left(P_{\alpha+1}-P_{\alpha}\right) Y, \omega_{0} \leq \alpha<\mu$, has an M-basis. Finally, a standard argument gives an M-basis on $Y$ (see [Fab97, Proposition 6.2.4]).

This result is less general than the one stated in Corollary 55. However, to prove it we did not need the full strength of Theorem 50 .

Remark 53 It is worth to observe that, in Theorem 50, (iv) implies (ii) in a very natural way. Indeed, the couple $(X, \Phi)$, where the mapping $\Phi$ is defined by $\Phi\left(x^{*}\right):=$ $\left\{\gamma \in G ;\left\langle\gamma, x^{*}\right\rangle \neq 0\right\}$ for $x^{*} \in X^{*}$, is a (full) PG. In order to see this, let $W$ be a subset of $X^{*}$ such that $\operatorname{span}_{\mathbb{Q}}(W)=W$. Let $x^{*} \in \bar{W}^{w^{*}} \cap \Phi(W)^{\perp}$. Assume that $x^{*} \neq 0$. Then, due to the linear density of $G$, we can find $\gamma \in G$ such that $\left\langle\gamma, x^{*}\right\rangle \neq 0$. As $x^{*} \in \bar{W}^{w^{*}}$, there is $w \in W$ such that $\langle\gamma, w\rangle \neq 0$. Then $\gamma \in \Phi(w) \subset \Phi(W)$. Thus $\left\langle\gamma, x^{*}\right\rangle=0$, and we reach a contradiction. This means that $\bar{W}^{w^{*}} \cap \Phi(W)^{\perp}=\{0\}$.

As a consequence of Proposition 47 and Theorem 50 we get the following well-known result.

\section{Corollary 54 Every WCD Banach space is WLD.}

A subspace $Y$ of a WLD Banach space $X$ is itself WLD. This is a consequence of the fact that the continuous image of a Corson compactum is again Corson compact, a deep result of Gul'ko [Gul79] and, independently, of Valdivia [Val91]. As a consequence of Theorem 50 we can obtain directly, without relying on that result, that a subspace of a WLD Banach space is again WLD (see Corollary 55). This fact has been also proved in [FGMZ04]; the proof we present here is yet simpler.

A compact topological space $K$ is angelic if for every non-empty subset $A$ of $K$, every element in $\bar{A}$ is the limit of a sequence in $A$ (a general definition of angelicity will be given in the paragraph prior to Proposition 118). We shall use the following fact. Let $f: K \rightarrow T$ be a continuous onto mapping, $K$ an angelic compact space, and $T$ a compact space. Then $T$ is also angelic. Indeed, let $\emptyset \neq B \subset T$. The family 
$\mathcal{A}:=\{A \subset K ; A$ closed, $f(A)=\bar{B}\}$ is non-empty and has, by Zorn's Lemma, a minimal element $A$, say. Let $A_{0}:=\{a \in A ; f(a) \in B\}$. Observe that $f\left(A_{0}\right) \subset B$ by the definition of $A_{0}$. If $b \in B$, then there exists $a \in A$ such that $b=f(a)$. Hence $a \in A_{0}$. Therefore $f\left(A_{0}\right)=B$. Now, $\bar{B}=\overline{f\left(A_{0}\right)}=f\left(\overline{A_{0}}\right) \subset f(A)=\bar{B}$, hence $f\left(\overline{A_{0}}\right)=\bar{B}$. Note that $\overline{A_{0}} \subset A$ and that $A$ is minimal. It follows that $\overline{A_{0}}=A$. Given $b \in \bar{B}$ there exists $a \in A$ such that $f(a)=b$ and, by angelicity, there exists a sequence $\left(a_{n}\right)$ in $A_{0}$ such that $a_{n} \rightarrow a$. It follows that $\left(b_{n}\right)\left(:=f\left(a_{n}\right)\right)$ is a sequence in $B$ which converges to $b$.

Corollary 55 Let $X$ be a WLD Banach space. Then, every subspace $Y$ of $X$ is again $W L D$.

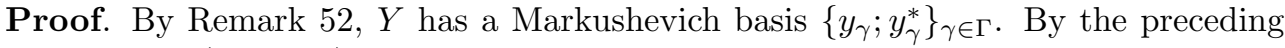
observation, $\left(B_{Y^{*}}, w^{*}\right)$ is angelic and therefore, by the Banach-Dieudonné Theorem, $\left\{y_{\gamma} ; \gamma \in \Gamma\right\}$ countably supports $Y^{*}$ (this argument was already used in the proof of (ii) $\Rightarrow$ (iii) in Theorem 50). It follows from Theorem 50 that $Y$ is WLD.

Remark 56 Under Martin's Axiom $\left(\mathrm{MA}_{\omega_{1}}\right)$, every Corson compactum has property (M), a result of Archangelskii, Šapirovskii and Kunen (see, for example, [Frem84] and [HMVZ07]). If this is the case, from Corollary 55 we obtain that the continuous image of a Corson compactum is again Corson.

We have the following simple consequence of Theorem 50 and Proposition 1 in [FGMZ04] (see [Val91] and [Vand95]). An M-basis $\left\{x_{\gamma} ; x_{\gamma}^{*}\right\}_{\gamma \in \Gamma}$ in a Banach space $X$ is called norming if the set $\left\{x_{\gamma}^{*} ; \gamma \in \Gamma\right\}$ is norming. The concept of norming subset was introduced in the paragraph prior to Definition 21. A set $\left\{x_{\gamma} ; \gamma \in \Gamma\right\}$ is called uniformly minimal if it is minimal (see the paragraph prior to Proposition 17) and the corresponding system $\left\{x_{\gamma} ; x_{\gamma}^{*}\right\}_{\gamma \in \Gamma}$ is bounded, i.e., there exists $M>0$ such that $\left\|x_{\gamma}\right\| .\left\|x_{\gamma}^{*}\right\| \leq M$ for all $\gamma \in \Gamma$.

Corollary 57 Let $X$ be a WLD Banach space and let $Y$ be a closed subspace of $X$. Then, every M-basis (resp. norming M-basis, resp. uniformly minimal M-basis) $\left\{y_{\gamma} ; y_{\gamma}^{*}\right\}_{\gamma \in \Gamma}$ on $Y$ can be extended to an M-basis (resp. norming M-basis, resp. uniformly minimal $M$-basis) on $X$.

Proof. Proceed by induction on the density character of $X$ : if $X$ is separable, the result follows from [GuKa62] (resp. [Ter87], resp. [Ter83]). Assume that the theorem has been proved for all WLD Banach spaces of density $<\aleph$, for some uncountable cardinal number $\aleph$. Suppose that $X$ is a WLD Banach space of density $\aleph$. Let $\left\{y_{\gamma} ; y_{\gamma}^{*}\right\}_{\gamma \in \Gamma}$ an M-basis on $Y$. Let $G$ be a total subset of $X$ countably supporting $X^{*}$. Then $\left\{y_{\gamma} ; \gamma \in \Gamma\right\} \cup G$ is a total subset of $X$ and countably supports $X^{*}$. By Proposition 30 there exists $\left(P_{\alpha}\right)_{\omega \leq \alpha \leq \mu}$, a PRI on $X$ subordinated to $\left\{y_{\gamma} ; \gamma \in \Gamma\right\} \cup G$. Using the aforesaid PRI, and letting $Q_{\alpha}:=P_{\alpha+1}-P_{\alpha}, \omega \leq \alpha<\mu$, extend the Mbasis $\left\{Q_{\alpha} y_{\gamma} ; \gamma \in \Gamma\right\}$ of $Q_{\alpha} Y$ to an M-basis (resp. norming M-basis, resp. uniformly minimal M-basis) of $Q_{\alpha}(X)$. Now, using [Fab97, Proposition 6.2.4], "glue together" 
those M-basis in one on $X$, which becomes an extension of $\left\{y_{\gamma} ; y_{\gamma}^{*}\right\}_{\gamma \in \Gamma}$ with the required properties.

Remark 58 Corollary 55 can be seen also as a simple consequence of Corollary 57 and the fact that a Markushevich basis $\left\{x_{\gamma} ; x_{\gamma}^{*}\right\}_{\gamma \in \Gamma}$ of a Banach space $X$ with a $w^{*}$ angelic dual unit ball has the property that $\left\{x_{\gamma} ; \gamma \in \Gamma\right\}$ countably supports all of $X^{*}$ (this follows from the Banach-Dieudonné Theorem; see again the proof of (ii) $\Rightarrow$ (iii) in Theorem 50).

We say that a Banach space is DENS if $\operatorname{dens} X=w^{*}$-dens $X^{*}$. Another simple consequence of Theorem 50 is the following well-known fact.

\section{Corollary 59 Every WLD Banach space is DENS.}

Proof. Let $\Gamma$ be a linearly dense subset of $X$ that countably supports $X^{*}$ (see Theorem 50), and let $D$ be a $w^{*}$-dense subset of $X^{*}$ with $\# D=w^{*}$-dens $X^{*}$. Given $x^{*} \in X^{*}$, put $\Gamma\left(x^{*}\right):=\left\{\gamma \in \Gamma ;\left\langle\gamma, x^{*}\right\rangle \neq 0\right\}$ (the support of $x^{*}$ in $\Gamma$, a countable set). Let $\Gamma(D):=\bigcup_{d^{*} \in D} \Gamma\left(d^{*}\right)$. We claim that $\Gamma(D)$ is linearly dense in $X$. Indeed, let $x^{*} \in X^{*}$ such that $\left\langle\gamma, x^{*}\right\rangle=0$ for all $\gamma \in \Gamma(D)$. Let $\left(d_{\iota}^{*}\right)$ be a net in $D$ that $w^{*}$ converges to $x^{*}$. Given $\gamma \notin \Gamma(D)$, we have $(0=)\left\langle\gamma, d_{\iota}^{*}\right\rangle \rightarrow\left\langle\gamma, x^{*}\right\rangle$, hence $\left\langle\gamma, x^{*}\right\rangle=0$, so $x^{*}=0$. This proves the claim.

Finally,

$$
\operatorname{dens} X \leq \# \Gamma(D)=\# D=w^{*}-\operatorname{dens} X^{*} \leq \operatorname{dens} X .
$$

This finishes the proof.

Remark 60 Observe that a Banach space exists that is not DENS and it has an M-basis. A simple example is given by the space $\ell_{1}(c)$, where $c$ is the cardinal of the continuum. This space has an M-basis (indeed, a long Schauder basis), and it is isometric to a subspace of $\ell_{\infty}$. This can be seen as follows: the dual $X^{*}$ of every separable Banach space $X$ is isometric to a subspace of $\ell_{\infty}$, since the mapping $x^{*} \rightarrow$ $\left(\left\langle x_{n}, x^{*}\right\rangle\right)_{n \in \mathbb{N}}$ is an isometry whenever $\left(x_{n}\right)$ is a dense sequence in $B_{X}$. It follows that $(C[0,1])^{*}$ is isometric to a subspace of $\ell_{\infty}$. The space $\ell_{1}(c)\left(=\ell_{1}([0,1])\right)$ is isometric to a subspace of $C[0,1]^{*}$. The isometry is given by the mapping $\phi: \ell_{1}([0,1]) \rightarrow C[0,1]^{*}$, where $\phi(x)(f):=\sum_{t \in[0,1]} x(t) f(t)$ for $f \in C[0,1]$. Finally, we get that $\ell_{1}(c)$ is isometric to a subspace of $\ell_{\infty}$. By Goldstine's theorem, $\left(\ell_{\infty}\right)^{*}$ is $w^{*}$-separable, so it is its quotient $\left(\ell_{1}(c)\right)^{*}$, and we get that $\ell_{1}(c)$ is not DENS.

The next theorem completes the information given in [Rych04], and depends essentially upon the following Valdivia's result in [Val96]: (a) Let $X$ be an Asplund space. Then $X$ has a biorthogonal system $\left\{x_{\gamma} ; x_{\gamma}^{*}\right\}_{\gamma \in \Gamma}$ such that $\overline{\operatorname{span}} w^{*}\left\{x_{\gamma}^{*} ; \gamma \in \Gamma\right\}=X^{*}$ and, moreover, $\left\{x_{\gamma}, x_{\gamma \mid E}^{*}\right\}_{\gamma \in \Gamma}$ is a shrinking Markushevich basis in $E$, where $E:=$ $\overline{\operatorname{span}}\left\{x_{\gamma} ; \gamma \in \Gamma\right\}$.

We say that a Banach space is $\langle F\rangle$ if it admits an equivalent Fréchet differentiable norm. 
Theorem 61 Let $E$ be a Banach space. Then, the following assertions are equivalent:

(i) There is a subspace $X \subset E$ with a shrinking Markushevich basis.

(ii) There is a subspace $Y \subset E$ which is Asplund and WCG.

(iii) There is a subspace $Z \subset E$ which is Asplund and DENS.

(iv) There is a subspace $U \subset E$ which is Asplund.

(v) There is a subspace $V \subset E$ which is $\langle F\rangle$ and $W C G$.

(vi) There is a subspace $W \subset E$ which is $\langle F\rangle$ and DENS.

(vii) There is a subspace $H \subset E$ which is $\langle F\rangle$.

Proof. (i) $\Rightarrow$ (ii) Every Banach space with a shrinking Markushevich basis is Asplund and WCG (and conversely, see, for example, [Fab97, p. 112 and Theorem 8.3.3]).

(ii) $\Rightarrow$ (iii) Every WCG space is WLD. Apply now Corollary 59.

(iii) $\Rightarrow$ (iv) is trivial.

(iv) $\Rightarrow$ (i) follows from Valdivia's result (a).

(i) $\Rightarrow$ (v) See, for example, [FHHMPZ01, Theorem 11.23].

(v) $\Rightarrow$ (vi) follows again from Corollary 59 .

(vi) $\Rightarrow$ (vii) is trivial.

(vii) $\Rightarrow$ (iv) Every $\langle F\rangle$ space is Asplund (see, for example, [FHHMPZ01, Cor. 10.9])

Remark 62 As it is well-known (see, for example, [Fab97, Theorem 8.3.3], in the framework of Asplund spaces all concepts WCG, subspace of WCG, WCD and WLD coincide. However, this is not the case with the concept DENS: the Banach space $C\left[0, \omega_{1}\right]$ is DENS (see, for example, [Z03]), Asplund ([0, $\left.\omega_{1}\right]$ is scattered) but not WLD $\left(\left[0, \omega_{1}\right]\right.$ is not Corson).

If $K$ is the Kunen compactum (see, for example, [HMVZ07]) then $C(K)$ is Asplund, not WLD ( $K$ is not Corson) and no non-separable subspace of $C(K)$ has a Markushevich basis (see, for example, [Z03]).

\subsection{Biorthogonal systems in WCG Banach spaces}

Our goal now is to present a remarkable tool (Theorem 71), an extension of a result provided by S. Argyros and S. Mercourakis in [ArgMe] that, among some other things, allows to check whether a Banach space $X$ having a Markushevich basis is WCG. It is based in a certain behavior of the coefficient functionals of the M-basis. What is amazing is that this property is shared by each of the existing M-bases. It will be instrumental to prove, in particular, an important theorem due to W. B. Johnson (Theorem 75) about unconditional long Schauder bases in WCG Banach spaces. The proof proposed of the aforesaid result of Argyros and Mercourakis is different (and we believe easier) than the original one - and indeed allow us to prove an extension of the original result. It is based on the existence of a certain projectional resolution of the identity. We shall use the result, too, to give a new proof of the fact that a 
certain subspace of a WCG space of from $C(K)$ is not itself WCG. This is, again, a very interesting result due to Argyros and Mercourakis [ArgMe].

In order to motivate Theorem 71, let us present some easy facts about biorthogonal systems in Banach spaces. Although they are not very deep, we did not see them described in the literature, and be believe that they provide the right motivation to the result mentioned above. First, we isolate a property of sets that plays an important role in the study of the structure of WCG Banach spaces, and that was used by Amir and Lindenstrauss in their seminal paper [AmLi68].

Definition 63 We say that a subset $\Gamma$ of a Banach space $X$ has the AmirLindenstrauss property (the (AL)-property, in short), if for every $x^{*} \in X^{*}$ and every $c>0$, the set $\left\{\gamma \in \Gamma ;\left|\left\langle\gamma, x^{*}\right\rangle\right|>c\right\}$ is finite.

Proposition 64 Let $X$ be a Banach space. A set $\Gamma \subset X$ with the (AL)-property countably supports $X^{*}$, and moreover, the set $\Gamma \cup\{0\}$ is weakly compact.

Proof. Let $x^{*} \in X^{*}$. The set $\Gamma_{n}:=\left\{\gamma \in \Gamma ;\left|\left\langle\gamma, x^{*}\right\rangle\right|>1 / n\right\}$ is finite for every $n \in \mathbb{N}$. Since $\left\{\gamma \in \Gamma ;\left\langle\gamma, x^{*}\right\rangle \neq 0\right\}=\bigcup_{n=1}^{\infty} \Gamma_{n}$, the conclusion follows. The uniform boundedness principle yields that the set $\Gamma$ is bounded. Let $\gamma^{* *}$ be in $\overline{\Gamma \cup\{0\}} w^{*} \backslash X$. Find $x^{*} \in X^{*}$ such that $\left\langle\gamma^{* *}, x^{*}\right\rangle>c>0$. Then the set $\left\{\gamma \in \Gamma ;\left\langle\gamma, x^{*}\right\rangle>c\right\}$ is infinite, a contradiction.

Remark 65 Let $\left\{x_{\lambda} ; f_{\lambda}\right\}_{\lambda \in \Lambda}$ be a total biorthogonal system in $X \times X^{*}$ (i.e., a biorthogonal system such that $\left\{f_{\lambda} ; \lambda \in \Lambda\right\}$ is $w^{*}$-linearly dense, see Definition 16). Then the only possible $w$-accumulation point in $X$ of the set $\left\{x_{\lambda} ; \lambda \in \Lambda\right\}$ is 0 . This is easy to prove: if there exists a net (of distinct points) $\left(x_{\lambda_{\iota}}\right)_{\iota \in I}$ in $\left\{x_{\lambda} ; \lambda \in \Lambda\right\}$ that $w$-converges to some point $x \in X$ then, obviously, $\left\langle x, f_{\lambda}\right\rangle=0$ for all $\lambda \in \Lambda$, so $x=0$.

The following proposition is now almost trivial.

Proposition 66 Let $\left\{x_{\lambda} ; f_{\lambda}\right\}_{\lambda \in \Lambda}$ be a total biorthogonal system in $X \times X^{*}$. Then the following are equivalent:

(i) The set $\left\{x_{\lambda} ; \lambda \in \Lambda\right\}$ has the (AL) property.

(ii) The set $\left\{x_{\lambda} ; \lambda \in \Lambda\right\}$ is weakly relatively compact (and so $\left\{x_{\lambda} ; \lambda \in \Lambda\right\} \cup\{0\}$ is weakly compact).

Proof. (i) $\Rightarrow$ (ii) follows from Proposition 64 and Remark 65.

(ii) $\Rightarrow$ (i). Assume that the set $\left\{x_{\lambda} ; \lambda \in \Lambda\right\}$ does not have the (AL)-property. Then there exists $x^{*} \in X^{*}$ and $c>0$ such that the set $\left\{\lambda \in \Lambda ;\left\langle x_{\lambda}, x^{*}\right\rangle>c\right\}$ is infinite. Since this set is weakly relatively compact, it has an accumulation point in $X$, say $x$ $(\neq 0)$. This is impossible in view of Remark 65 .

The following simple proposition is a consequence of the orthogonality. 
Proposition 67 Let $X$ be a Banach space. Let $\left\{x_{i} ; f_{i}\right\}_{i \in \mathbb{N}}$ be a biorthogonal system in $X \times X^{*}$ and assume that $\left(\sum_{i=1}^{n} f_{i}\right)_{n \in \mathbb{N}}$ has a bounded subsequence. Then $\left\{x_{i} ; i \in \mathbb{N}\right\}$ lies in a hyperplane missing 0.

Proof. Let $\left(n_{p}\right)$ be an increasing sequence in $\mathbb{N}$ such that the sequence $\left(\sum_{i=1}^{n_{p}} f_{i}\right)_{p=1}^{\infty}$ is bounded and let $x^{*}$ be a $w^{*}$-cluster point of it. Then, for every $j \in \mathbb{N}$ we have

$$
\left\langle x_{j}, \sum_{i=1}^{n_{p}} f_{i}\right\rangle=1 \text { for all large } p \in \mathbb{N} \text {. }
$$

Then $\left\langle x_{j}, x^{*}\right\rangle=1$ for all $j \in \mathbb{N}$.

Corollary 68 Let $X$ be a Banach space and let $\left\{x_{\lambda} ; f_{\lambda}\right\}_{\lambda \in \Lambda}$ be a biorthogonal system in $X \times X^{*}$. Assume that $\left\{x_{\lambda} ; \lambda \in \Lambda\right\}$ has the (AL)-property. Then, for every oneto-one sequence $\left(\lambda_{n}\right)$ in $\Lambda$ we have $\left\|\sum_{i=1}^{n} f_{\lambda_{i}}\right\| \rightarrow \infty$ whenever $n \rightarrow \infty$.

Proof. If the conclusion does not hold for some one-to-one sequence $\left(\lambda_{n}\right)$, there is an increasing sequence $\left(n_{p}\right)$ such that $\left(\sum_{i=1}^{n_{p}} f_{\lambda_{i}}\right)_{p=1}^{\infty}$ is bounded. It follows from Proposition 67 that $\left\{x_{\lambda_{n}} ; n \in \mathbb{N}\right\}$ is in a hyperplane missing 0 , and this violates the (AL)-property.

Theorem 71 below is an extension of a result obtained by Argyros and Mercourakis. To prove their result, they used a combinatorial lemma (see [Fab97, Lemma 1.6.2]) due to Argyros. Here we show an alternative argument for our more general result based on the construction of a separable PRI, inspired in Proposition 67. Again, orthogonality plays the key role.

We believe that the origin of Theorem 71 can be traced to the following theorem, due to V. Pták.

We recall here that a biorthogonal system $\left\{x_{\gamma} ; x_{\gamma}^{*}\right\}_{\gamma \in \Gamma}$ in $X \times X^{*}$ is called bounded if there exists a constant $M>0$ such that $\left\|x_{\gamma}\right\| \cdot\left\|x_{\gamma}^{*}\right\| \leq M$ for all $\gamma \in \Gamma$. The infimum of those constants is called the boundedness constant, and we say that a biorthogonal system $\left\{x_{\gamma} ; x_{\gamma}^{*}\right\}_{\gamma \in \Gamma}$ is $M$-bounded if $M$ is its boundedness constant.

It is worth mentioning that a deep theorem due to Pełczyński and Plichko says that, for every $\varepsilon>0$, every separable Banach space has a $(1+\varepsilon)$-bounded Markushevich basis (see, e.g., [HMVZ07] and the references therein). This result was somehow extended by Plichko to WCG Banach spaces (the constant was now $(2+\varepsilon)$ ). He claimed, too, that the result hold, with a larger constant, for every Banach space having an M-basis, although his proof had a serious flaw. In a recent preprint [HM], it has been proved that, for every $\varepsilon>0$, every Banach space with an M-basis has another $2(1+\sqrt{2})+\varepsilon$-bounded M-basis.

We give here a slightly more precise formulation of Pták's result.

Theorem 69 (Pták, [Pt59]) Let $X$ be a Banach space. The following statements are equivalent. 
(i) $X$ is reflexive.

(ii) For every biorthogonal system $\left\{x_{n} ; x_{n}^{*}\right\}_{n=1}^{\infty}$ in $X \times X^{*}$ such that $\left\{x_{n}^{*}\right\}_{n=1}^{\infty}$ is bounded, the sequence $\left(\sum_{k=1}^{n} x_{k}\right)_{n \in N}$ is unbounded.

(iii) For every biorthogonal system $\left\{x_{n} ; x_{n}^{*}\right\}_{n=1}^{\infty}$ in $X \times X^{*}$ such that $\left\{x_{n}\right\}_{n=1}^{\infty}$ is bounded, the sequence $\left(\sum_{k=1}^{n} x_{k}^{*}\right)_{n \in \mathbb{N}}$ is unbounded.

We quote in the next three paragraphs the reviewer (B. R. Gelbaum) of the original paper in MathSciNet.

The original proof is based on the following intermediate results.

For a Banach space $X$ the following statements are equivalent: (a) $X$ is non-reflexive. (b) There is a bounded biorthogonal system $S_{1}=\left\{e_{i} ; f_{i}\right\}_{i \in \mathbb{N}}$ and $a \Delta>0$ such that if $\alpha_{n} \uparrow 0$ or if $\alpha_{n} \downarrow 0$ then $x=\sum_{i=1}^{\infty} \alpha_{i} e_{i}$ exists and $\|x\| \leq \Delta\left|\alpha_{1}\right|$. (c) Let $B(S)=w^{*}$-closure of $f_{j}$ in $E^{*}$. There exists a bounded biorthogonal system $S_{2}$ such that, considered as a biorthogonal system in $X / B\left(S_{2}\right)^{0}, S_{2}$ enjoys the property: there is a $\Delta>0$ such that if $0 \leq \lambda_{i}, \sum_{i=1}^{\infty} \lambda_{i}<\infty$, then $\sum_{i=1}^{\infty} \lambda_{i} e_{i} \geq \Delta \sum_{i=1}^{\infty} \lambda_{i}$.

The key to Theorem 69 lies in constructing for a given non-reflexive $X$ a system $S$ for which (b) and (c) are true. Once this is achieved, the remaining syllogisms follow readily. The root idea is then the following. Choose $r \in X^{* *} \backslash X$. Then for $\delta>0$, by induction construct sequences $\left\{b_{i}\right\} \subset X,\left\{y_{j}\right\} \subset X^{*}$ such that $\left\|b_{i}\right\| \leq 1+\delta,\left\|y_{j}\right\|=1,\left(b_{i}, y_{j}\right)=\beta_{j}>$ $\frac{1}{2} \delta_{j}>0$ for $j \leq i,\left(b_{i}, y_{j}\right)=0$ for $j>i$, where $\delta_{j}=\sup \{(r, y) \mid\|y\| \leq 1$, $y \in X_{j-1}=$ linear space spanned by $\left.b_{1}, b_{2}, \cdots, b_{j-1}\right\}$. Then there is a $\beta>0$ such that $\delta_{j} \geq 2 \beta$. Set $e_{i}=b_{i}-b_{i-1}, f_{j}=\left(1 / \beta_{j}\right) y_{j} .\left\{e_{i} ; f_{j}\right\}$ is a system $S_{1}$ for (b). Set $g_{i}=b_{i}, h_{j}=\left(1 / \beta_{j}\right) y_{j}-\left(1 / \beta_{j+1}\right) y_{j+1} \cdot\left\{g_{i} ; h_{j}\right\}$ is a system $S_{2}$ for (c). $S_{1}$ is also a system for which the sums $\sum_{i=1}^{n} e_{i}, n \in \mathbb{N}$, are bounded. The remaining arguments are less involved.

We shall present here a different approach, based on a well-known characterization of reflexivity due to James. James' result depends, essentially, on Helly's Theorem (for a proof of the equivalence between (i) and (iii) below, based only on Riesz' Lemma, we refer to [FHHMPZ01, Theorem 3.57]). We recall here this characterization, as it is presented, for example, in [Beau82, Theorem III.6].

Theorem 70 Let $X$ be a Banach space. The following are equivalent.

(i) $X$ is not reflexive.

(ii) For every $0<\theta<1$, there is a sequence $\left(x_{n}\right)$ in $S_{X}$ and a sequence $\left(x_{n}^{*}\right)$ in $S_{X^{*}}$ such that

$$
\begin{cases}\left\langle x_{n}, x_{m}^{*}\right\rangle=\theta & \text { for all } n \geq m, \\ \left\langle x_{n}, x_{m}^{*}\right\rangle=0 & \text { for all } n<m .\end{cases}
$$


(iii) For every $0<\theta<1$, there is a sequence $\left(x_{n}\right)$ in $S_{X}$ such that $\inf \left\{\|u\| ; u \in \operatorname{conv}\left\{x_{n} ; n \in \mathbb{N}\right\}\right\} \geq \theta$ and

$$
\operatorname{dist}\left(\operatorname{conv}\left\{x_{k}\right\}_{k=1}^{n}, \operatorname{conv}\left\{x_{k}\right\}_{k=n+1}^{\infty}\right) \geq \theta \quad \text { for all } \quad n \in \mathbb{N} .
$$

Proof of Theorem 69. (i) $\Rightarrow$ (ii) and (iii). Assume that the space $X$ is reflexive. Let $\left\{x_{n} ; x_{n}^{*}\right\}_{n \in \mathbb{N}}$ be a biorthogonal system in $X \times X^{*}$ with $\left\{x_{n}\right\}_{n=1}^{\infty}$ bounded. Let $Y:=\operatorname{span}\left\{x_{n} ; n \in \mathbb{N}\right\}$; this is a reflexive space. Let $q: X^{*} \rightarrow Y^{*}$ be the restriction mapping. Then $\left\{x_{n} ; q\left(x_{n}^{*}\right)\right\}$ is a biorthogonal system in $Y \times Y^{*}$. And, as $Y$ is reflexive, the sets $\left\{x_{1}, x_{2}, \ldots\right\} \cup\{0\}$ and $\left\{q\left(x_{1}^{*}\right), q\left(x_{2}^{*}\right), \ldots\right\} \cup\{0\}$ are both weakly compact. Since $\left\{q\left(x_{n}^{*}\right) ; x_{n}\right\}$ is a total biorthogonal system in $Y^{*} \times Y$, Proposition 66 and Corollary 68 give (ii). Reversing the roles of $X$ and $X^{*}$ we get (iii).

(ii) $\Rightarrow$ (i) Assume that $X$ is not reflexive. Theorem 70 says that, given $0<\theta<1$, there exist two sequences, $\left(x_{n}\right)$ in $S_{X}$ and $\left(x_{n}^{*}\right)$ in $S_{X^{*}}$, such that $\left\langle x_{n}, x_{m}^{*}\right\rangle=\theta$ if $n \geq m$, and $\left\langle x_{n}, x_{m}^{*}\right\rangle=0$ if $n<m$. Let $d_{1}:=x_{1}, d_{n}:=x_{n}-x_{n-1}, n=2,3, \ldots$ Then, it is clear that the family $\left\{(1 / \theta) d_{n} ; x_{n}^{*}\right\}_{n \in \mathbb{N}}$ is a biorthogonal system in $X \times X^{*}$. Moreover, $\left\{x_{n}^{*} ; n \in \mathbb{N}\right\}$ is bounded. Observe, too, that $\sum_{k=1}^{n} d_{k}=x_{n}$ for all $n \in \mathbb{N}$. We obtain a contradiction with (ii).

(iii) $\Rightarrow$ (i) Starting from the assumption that $X$ is not reflexive, we proceed as in the proof of (ii) $\Rightarrow(\mathrm{i})$. Once we have the two sequences $\left(x_{n}\right)$ and $\left(x_{n}^{*}\right)$, put $d_{n}^{*}=x_{n}^{*}-x_{n+1}^{*}$ for $n \in \mathbb{N}$. The system $\left\{x_{n} ;(1 / \theta) d_{n}^{*}\right\}_{n \in \mathbb{N}}$ is again a biorthogonal system such that $\left\{x_{n} ; n \in \mathbb{N}\right\}$ is bounded, and now $\sum_{k=1}^{n} d_{n}^{*}=x_{1}^{*}-x_{n+1}^{*}$ for all $n \in \mathbb{N}$. We obtain again a contradiction, this time with (iii).

We shall prove now our extension (Theorem 71) of the Argyros and Mercourakis result cited in Corollary 73. We do not request that the space should be WCG, neither that the biorthogonal system should be an M-basis.

Theorem 71 Let $X$ be a Banach space. Let $K \subset X$ be a non-empty weakly compact set, and let $\left\{x_{\lambda} ; f_{\lambda}\right\}_{\lambda \in \Lambda}$ be a fundamental biorthogonal system in $X \times X^{*}$. Let $\Lambda^{0}:=$ $\left\{\lambda \in \Lambda ;\left\langle k, f_{\lambda}\right\rangle \neq 0\right.$ for some $\left.k \in K\right\}$. Then there exists a splitting $\Lambda^{0}=\bigcup_{m=1}^{\infty} \Lambda_{m}^{0}$ such that $\lim _{n \rightarrow \infty}\left\|\sum_{i=1}^{n} f_{\lambda_{i}}\right\|=+\infty$ for every fixed $m \in \mathbb{N}$ and every one-to-one sequence $\lambda_{1}, \lambda_{2}, \ldots \in \Lambda_{m}^{0}$.

Proof. Let $Y:=\overline{\operatorname{span}}(K)$, a WCG subspace of $X$. By a well-known result of Amir and Lindenstrauss [AmLi68], there is a linearly dense set $\Gamma \subset B_{Y}$ with property (AL); see, for instance [FGMZ04, Theorem 1] or just combine Corollary 44 and Proposition 66. Let $q: X^{*} \rightarrow Y^{*}$ be the canonical quotient mapping. Put $\Delta^{0}:=\left\{q f_{\lambda} ; \lambda \in\right.$ $\left.\Lambda^{0}\right\}$. Observe that $q f_{\lambda}=0$ for $\lambda \in \Lambda \backslash \Lambda^{0}$. The set $\Delta^{0}$ countably supports $Y$ (see Remark 25). It is clear, too, that $\Gamma$ countably supports $Y^{*}$ (see Proposition 64). Let $\left(P_{\alpha}\right)_{\omega_{0} \leq \alpha \leq \mu}$ be a SPRI on $Y$ found for these $\Gamma$ and $\Delta^{0}$ by Proposition 41 .

Fix $\alpha \in\left[\omega_{0}, \mu\right)$. Put $Q_{\alpha}:=\left(P_{\alpha+1}-P_{\alpha}\right)$.

Claim: $\Delta_{\alpha}^{0}:=Q_{\alpha}^{*} Y^{*} \cap \Delta^{0}$ is countable (observe that, due to Lemma $27, \Delta^{0}=$ $\bigcup_{\omega_{0} \leq \alpha<\mu} \Delta_{\alpha}^{0}$ ). This can be seen as follows. Put $\Gamma_{\alpha}:=\Gamma \cap Q_{\alpha} Y$ (notice again that, 
due to Lemma 27, $\left.\Gamma=\bigcup_{\omega_{0}<\alpha<\mu} \Gamma_{\alpha}^{0}\right)$. Thanks to the fact that $\Gamma$ is subordinated to $\left(P_{\alpha}\right)_{\omega_{0} \leq \alpha \leq \mu}$, we have $Q_{\alpha} \bar{\Gamma}=\Gamma_{\alpha} \cup\{0\}$, and then $\Gamma_{\alpha}$ is linearly dense in (the separable space) $Q_{\alpha} Y$. We can find a countable dense subset $\Gamma_{\alpha}^{0}$ of $\Gamma_{\alpha}$. For $y \in Y$, let $\operatorname{supp}(y):=\left\{q f_{\lambda} ; \lambda \in \Lambda^{0},\left\langle y, f_{\lambda}\right\rangle \neq 0\right\}$. If $\gamma \in \Gamma_{\alpha}, \operatorname{supp}(\gamma)$ is a subset of $\Delta_{\alpha}^{0}$; indeed, for $q f_{\lambda} \in \operatorname{supp}(\gamma)$ we have $\left\langle\gamma, Q_{\alpha}^{*} q f_{\lambda}\right\rangle=\left\langle Q_{\alpha} \gamma, q f_{\lambda}\right\rangle=\left\langle\gamma, q f_{\lambda}\right\rangle \neq 0$, hence $Q_{\alpha}^{*} q f_{\lambda}=q f_{\lambda}$, since $\Delta^{0}$ is subordinated to $\left(Q_{\alpha}^{*}\right)_{\omega_{0} \leq \alpha \leq \mu}$. The set $\bigcup_{\gamma \in \Gamma_{\alpha}^{0}} \operatorname{supp}(\gamma)(\subset$ $\left.\Delta_{\alpha}^{0}\right)$ is countable. Let us see that $\Delta_{\alpha}^{0}=\bigcup_{\gamma \in \Gamma_{\alpha}^{0}} \operatorname{supp}(\gamma)$; given $q f_{\lambda} \in \Delta_{\alpha}^{0}$, we have $q f_{\lambda} \neq 0$, so there exists $\gamma \in \Gamma_{\alpha}^{0}$ such that $\left\langle\gamma, q f_{\lambda}\right\rangle \neq 0$, and thus $q f_{\lambda} \in \operatorname{supp}(\gamma)$. This proves the Claim.

Let $\left\{f_{\alpha}^{m} ; m \in \mathbb{N}\right\}$ be an enumeration of the set $\Delta_{\alpha}^{0}$. The set $Q_{\alpha} \Gamma$ is linearly dense in $Q_{\alpha} Y$. Then, for each $f_{\alpha}^{m}$ we can find an element $\gamma_{\alpha}^{m} \in Q_{\alpha} \Gamma$ such that $\left\langle\gamma_{\alpha}^{m}, \delta_{\alpha}^{m}\right\rangle \neq 0$, $n \in \mathbb{N}$. Put

$$
\Lambda_{m}^{0}=\left\{\lambda \in \Lambda^{0} ; f_{\lambda}=f_{\alpha}^{m} \text { for some } \alpha \in\left[\omega_{0}, \mu\right)\right\}, \quad m \in \mathbb{N} .
$$

Clearly, $\bigcup_{m=1}^{\infty} \Lambda_{m}^{0}=\Lambda^{0}$. Further, for $m \in \mathbb{N}$ and $l \in \mathbb{N}$ put

$$
\Lambda_{m, l}^{0}:=\left\{\lambda \in \Lambda_{m}^{0} ; f_{\lambda}=f_{\alpha}^{m} \text { and }\left|\left\langle\gamma_{\alpha}^{m}, f_{\alpha}^{m}\right\rangle\right|>\frac{1}{l} \text { for some } \alpha \in\left[\omega_{0}, \mu\right)\right\} .
$$

Clearly, $\bigcup_{m, l=1}^{\infty} \Lambda_{m, l}^{0}=\Lambda^{0}$.

Now fix any $m, l \in \mathbb{N}$ and consider a one-to-one sequence $\lambda_{1}, \lambda_{2}, \ldots$ in $\Lambda_{m, l}^{0}$. Find $\alpha_{1}, \alpha_{2}, \ldots$ in $\left[\omega_{0}, \mu\right)$ so that $f_{\lambda_{i}}=f_{\alpha_{i}}^{m}, i \in \mathbb{N}$. We shall show that $\lim _{n \rightarrow \infty}\left\|\sum_{i=1}^{n} f_{\lambda_{i}}\right\|=$ $+\infty$. Assume not. Let $y^{*} \in Y^{*}$ be a $w^{*}$-cluster point of the sequence $\left(\sum_{i=1}^{n} f_{\lambda_{i}}\right)_{n \in \mathbb{N}}$. For any fixed $k \in \mathbb{N}$, we then have

$$
\begin{aligned}
& \left|\left\langle\gamma_{\alpha_{k}}^{m}, y^{*}\right\rangle\right| \geq \liminf _{n \rightarrow \infty}\left|\left\langle\gamma_{\alpha_{k}}^{m}, \sum_{i=1}^{n} f_{\lambda_{i}}\right\rangle\right| \\
& \quad=\liminf _{n \rightarrow \infty}\left|\left\langle\gamma_{\alpha_{k}}^{m}, \sum_{i=1}^{n} f_{\alpha_{i}}^{m}\right\rangle\right|=\left|\left\langle\gamma_{\alpha_{k}}^{m}, f_{\alpha_{k}}^{m}\right\rangle\right|>\frac{1}{l}
\end{aligned}
$$

(the last equality due to the fact that the "long sequence" of subspaces $\left(Q_{\alpha} Y\right)_{\omega_{0} \leq \alpha<\mu}$ is "orthogonal"). But the sequence $\gamma_{\alpha_{1}}^{m}, \gamma_{\alpha_{2}}^{m}, \ldots$ is one-to-one. Thus we get a contradiction with the $(\mathrm{AL})$ property of the set $\Gamma$.

Corollary 72 Let $X$ be a WCG Banach space, and let $\left\{x_{\lambda} ; f_{\lambda}\right\}_{\lambda \in \Lambda}$ be a fundamental biorthogonal system in $X \times X^{*}$. Then there exists a splitting $\Lambda=\bigcup_{m=1}^{\infty} \Lambda_{m}$ such that $\lim _{n \rightarrow \infty}\left\|\sum_{i=1}^{n} f_{\lambda_{i}}\right\|=+\infty$ for every fixed $m \in \mathbb{N}$ and every one-to-one sequence $\lambda_{1}, \lambda_{2}, \ldots \in \Lambda_{m}$.

Proof. Since $X$ is WCG, there is a linearly dense and weakly compact subset $K$ of $X$. The set $\Lambda^{0}$ defined in Theorem 71 for this $K$ coincides with $\Lambda$. It is enough to apply Theorem 71 .

The following result, now simply a version for M-bases of Corollary 72, was proved originally by very different techniques. 
Corollary 73 (Argyros and Mercourakis, [ArgMe], Theorem 2.2) Let $X$ be a $W C G$ Banach space, and let $\left\{x_{\lambda} ; f_{\lambda}\right\}_{\lambda \in \Lambda}$ be an M-basis in $X \times X^{*}$. Then there exists a splitting $\Lambda=\bigcup_{m=1}^{\infty} \Lambda_{m}$ such that $\lim _{n \rightarrow \infty}\left\|\sum_{i=1}^{n} f_{\lambda_{i}}\right\|=+\infty$ for every fixed $m \in \mathbb{N}$ and every one-to-one sequence $\lambda_{1}, \lambda_{2}, \ldots \in \Lambda_{m}$.

It was observed in [ArgMe], without going into details, that Corollary 73 has, as an important consequence, a result of Johnson (Theorem 75).

In order to give a complete (and different) proof of it, we proceed in the following way. First we prove that our Theorem 71 implies a result of Argyros and Farmaki (Theorem 74). Then we prove that the result of Johnson (Theorem 75) follows from it. Johnson's result is, somehow, a converse to Corollary 68 under unconditionality, and it has been used by Rosenthal [Ros74] to prove that a WCG Banach space of form $L_{1}(\nu)$, with a certain measure $\nu$, has a subspace that is not WCG.

Let us stress that, in this way, Argyros and Mercourakis' (quoted in Corollary 73), Argyros and Farmaki's (Theorem 74) and Johnson's result (Theorem 75) are all consequence of our Theorem 71, proved only by using SPRI's. In the same way, Argyros' example of a subspace of a WCG $C(K)$ space that is not WCG (Example 77) is checked by using our Theorem 71 .

Theorem 74 ([ArFa], Lemma B) Let $X$ be a Banach space admitting an unconditional basis $\left\{x_{\lambda} ; f_{\lambda}\right\}_{\lambda \in \Lambda}$. Let $K \subset X$ be a non-empty weakly compact set. Let $\Lambda^{0}:=$ $\left\{\lambda \in \Lambda ;\left\langle k, f_{\lambda}\right\rangle \neq 0\right.$ for some $\left.k \in K\right\}$. Then there exists a splitting $\Lambda^{0}=\bigcup_{m=1}^{\infty} \Lambda_{m}^{0}$ such that for every $m \in \mathbb{N}$, the set $\left\{x_{\lambda} ; \lambda \in \Lambda_{m}^{0}\right\} \cup\{0\}$ is weakly compact.

Proof. Let $\Lambda_{m}^{0}, m \in \mathbb{N}$, be the sets found in Theorem 71 for our basis and for the given compact set $K$. Fix one $m \in \mathbb{N}$. We shall show that the only $w^{*}$-cluster point of the set $\left\{x_{\lambda} ; \lambda \in \Lambda_{m}^{0}\right\}$ in $X^{* *}$, is 0 . Assume this is not so. Find then $c>0, \xi \in S_{X^{*}}$ and a one-to-one sequence $\lambda_{1}, \lambda_{2}, \ldots \in \Lambda_{m}^{0}$ so that $\left|\left\langle x_{\lambda_{i}}, \xi\right\rangle\right|>c$ for every $i \in \mathbb{N}$. Then for every $n \in \mathbb{N}$, for every $a_{1}, \ldots, a_{n} \in \mathbb{R}$, and for suitable $\varepsilon_{1}, \ldots, \varepsilon_{n} \in\{-1,1\}$ we have

$$
\begin{aligned}
\left\|\sum_{i=1}^{n} a_{i} x_{\lambda_{i}}\right\| & \geq C\left\|\sum_{i=1}^{n} \varepsilon_{i} a_{i} x_{\lambda_{i}}\right\| \geq C\left\langle\sum_{i=1}^{n} \varepsilon_{i} a_{i} x_{\lambda_{i}}, \xi\right\rangle \\
& =C \sum_{i=1}^{n}\left|a_{i}\right|\left|\left\langle x_{\lambda_{i}}, \xi\right\rangle\right| \geq C \cdot c \sum_{i=1}^{n}\left|a_{i}\right| ;
\end{aligned}
$$

here $C$ denotes the "unconditional basis constant". (Therefore $X$ contains an isomor- 
phic copy of $\ell_{1}$.) We have then, for every $n \in \mathbb{N}$,

$$
\begin{aligned}
\left\|\sum_{i=1}^{n} f_{\lambda_{i}}\right\| & =\sup \left\{\left\langle x, \sum_{i=1}^{n} f_{\lambda_{i}}\right\rangle ; x \in \operatorname{span}\left\{x_{\lambda} ; \lambda \in \Lambda_{m}\right\},\|x\| \leq 1\right\} \\
& =\sup \left\{\left\langle\sum_{j=1}^{n} a_{j} x_{\lambda_{j}}, \sum_{i=1}^{n} f_{\lambda_{i}}\right\rangle ; a_{1}, \ldots, a_{n} \in \mathbb{R},\left\|\sum_{j=1}^{n} a_{j} x_{\lambda_{j}}\right\| \leq 1\right\} \\
& =\sup \left\{\sum_{i=1}^{n} a_{i} ; a_{1}, \ldots, a_{n} \in \mathbb{R},\left\|\sum_{j=1}^{n} a_{j} x_{\lambda_{j}}\right\| \leq 1\right\} \\
& \leq \sup \left\{\sum_{i=1}^{n} a_{i} ; a_{1}, \ldots, a_{n} \in \mathbb{R}, C \cdot c \sum_{j=1}^{n}\left|a_{j}\right| \leq 1\right\}=\frac{1}{C \cdot c}<+\infty .
\end{aligned}
$$

This contradicts the conclusion of Theorem 71 .

The following result is now a simple consequence of Theorem 74 .

Theorem 75 (Johnson, see, e.g., [Ros74]) Let X be a WCG Banach space admitting an unconditional basis $\left\{x_{\lambda} ; f_{\lambda}\right\}_{\lambda \in \Lambda}$. Then there exists a splitting $\Lambda=\bigcup_{m=1}^{\infty} \Lambda_{m}$ such that for every $m \in \mathbb{N}$, the set $\left\{x_{\lambda} ; \lambda \in \Lambda_{m}\right\} \cup\{0\}$ is weakly compact.

Remark 76 Theorem 71 cannot be extended to subspaces of WCG spaces. Indeed, if so, then Theorem 75 would also be extendable. However, Theorem 75 does not work for unconditional basic sequences. Indeed, Argyros and Mercourakis proved, in [ArgMe], that there is a WCG space $\mathrm{X}$ with unconditional basis and a subspace $\mathrm{Y}$ of $\mathrm{X}$ with unconditional basis such that $\mathrm{Y}$ is not WCG. The unconditional basis of $Y$ (an unconditional basic sequence in $X$ ) cannot be $\sigma$-weakly compact, since then $Y$ would be WCG.

To finalize this chapter, we present a simpler proof of the fact that a counterexample provided by Argyros to the so-called "heredity problem" for WCG (to know whether a subspace of a WCG Banach space must be WCG itself-a problem solved by Rosenthal in [Ros74]) satisfies the required properties. Since the behavior depends ultimately on Theorem 71, and this result was proved just by using the technology of PRI's, the counterexample of Argyros relies finally only on "elementary" arguments, contrary to Rosenthal's.

Example 77 Argyros' example of a subspace of $C(K)$ described in [Fab97, Section 1.6] is not $W C G$.

Proof. In the first part of this proof we follows the way the example is presented in $\left[\right.$ Fab97, Section 1.6]. Let $\Gamma:=\mathbb{N}^{\mathbb{N}}$. Given an element $\sigma \in \Gamma$ and some $n \in \mathbb{N}$, let us put $\sigma \mid n:=(\sigma(1), \sigma(2), \ldots, \sigma(n))$. For $n \in \mathbb{N}$, let $\mathcal{A}_{n}$ be the family of all $A \subset \Gamma$ with the following property: given distinct $\sigma, \tau$ in $A$, then $\sigma|n=\tau| n$, and $\sigma(n+1) \neq \tau(n+1)$. 
It is clear that each $A \in \mathcal{A}_{n}$ is countable. We shall consider the space $[0,1]^{\Gamma}$ endowed with the product topology.

Fix any $n \in \mathbb{N}$. We claim first that the set $\left\{\chi_{A} ; A \in \mathcal{A}_{n}\right\}\left(\subset[0,1]^{\Gamma}\right)$ is compact. Indeed, it is closed. This can be seen as follows: every point in its closure is of the form $\chi_{B}$ for some $B \subset \Gamma$. Let $\sigma \neq \tau$ in $B$. We can find $A \in \mathcal{A}_{n}$ such that $\{\sigma, \tau\} \subset A$. It follows that $\sigma|n=\tau| n$, and $\sigma(n+1) \neq \tau(n+1)$. $B$ has thus the property that makes it an element of $\mathcal{A}_{n}$.

Once this has been proved, the set

$$
K:=\bigcup_{n \in \mathbb{N}}\left\{\frac{1}{n} \chi_{A} ; A \in \mathcal{A}_{n}\right\} \cup\{0\},
$$

in $[0,1]^{\Gamma}$ is clearly compact. It is actually an Eberlein compact. In order to see this, let $x \in K$ be of the form $x=(1 / n) \chi_{A}$ with $n \in \mathbb{N}$ and $A \in \mathcal{A}_{n}$. We define $T x \in[0,1]^{\Gamma}$ as $T x(\sigma)=2^{-n} 3^{-\sigma(n+1)} \chi_{A}(\sigma)$ for $\sigma \in \Gamma$. Then, using the definition of $\mathcal{A}_{n}$, we get that $T x \in c_{0}(\Gamma)$. The continuity of $T$ (with respect to the weak topology of $c_{0}(\Gamma)$ ) is obvious. And since $T$ is also injective, we can conclude that $K$ is an Eberlein compact. It follows that the space $C(K)$ is WCG.

We shall prove now that $C(K)$ has a non-WCG closed subspace. To that end, we shall use Theorem 71 or, more precisely, Corollary 72. Our treatment from now on differs from (and simplifies) the original one.

For $\lambda \in \mathbb{N}^{\mathbb{N}}$ we define the evaluation function

$$
\pi_{\lambda}(k)=k(\lambda), \quad k \in K .
$$

Clearly, $\pi_{\lambda} \in C(K)$. Define then the Banach space $Y$ as the closed linear hull of $\left\{\pi_{\lambda} ; \lambda \in \mathbb{N}^{\mathbb{N}}\right\}$. For $\lambda \in \mathbb{N}^{\mathbb{N}}$ we further put

$$
f_{\lambda}(y)=y\left(\chi_{\{\lambda\}}\right), \quad y \in Y
$$

clearly $f_{\lambda} \in Y^{*}$. Then $\left\{\pi_{\lambda} ; f_{\lambda}\right\}_{\lambda \in \mathbb{N}^{\mathbb{N}}}$ is a fundamental biorthogonal system in $Y \times Y^{*}$. Assume that $Y$ is WCG. Let $\mathbb{N}^{\mathbb{N}}=\bigcup_{m=1}^{\infty} \Lambda_{m}$ be a partition found in Theorem 71 for our system. [Fab97, Lemma 1.6.1] yields $m, n \in \mathbb{N}$ and an infinite set $A=$ $\left\{\lambda_{1}, \lambda_{2}, \ldots\right\} \subset \Lambda_{m}$ such that $A \in \mathcal{A}_{n}$.

Now consider any $l \in \mathbb{N}$. We realize that $\frac{1}{n} \chi_{\left\{\lambda_{1}, \ldots, \lambda_{l}\right\}}$ belongs to the compact space $K$. Hence, for any element $y$ from the linear span of $\left\{\pi_{\lambda} ; \lambda \in \mathbb{N}^{\mathbb{N}}\right\}$ we have

$$
\left\langle y, \sum_{i=1}^{l} f_{\lambda_{i}}\right\rangle=\sum_{i=1}^{l} f_{\lambda_{i}}(y)=n \cdot y\left(\frac{1}{n} \chi_{\left\{\lambda_{1}, \ldots, \lambda_{l}\right\}}\right) \leq n\|y\|
$$

and so $\left\|\sum_{i=1}^{l} f_{\lambda_{i}}\right\| \leq n$ for every $l \in \mathbb{N}$. But this contradicts Theorem 71 . 


\section{Chapter 3}

\section{A renorming result}

In this short chapter we shall follow the approach used in Chapter 1, i.e., we shall particularize some well-known constructions to a more natural setting, namely to the case where a certain index set has some (topological) structure.

There, Gul'ko compacta were studied for subsets of products of the kind $\{0,1\}^{\Gamma}$, and even we restricted ourselves to the case when $\Gamma$ is a subset of the real line. Here, we produce, in a simple way, a rotund norm on $c_{0}(\Gamma)$, where, again, $\Gamma$ is a subset of $\mathbb{R}$. Of course, the result is superseded by the well-known Day's (and later on, Rainwater's) result. The value of our construction is its simplicity. We proclaim that our setting is quite natural, since the important cases for applications are modelled using our ingredients.

Let $\Gamma \subset \mathbb{R}$ be an uncountable set. Consider the Banach space $c_{0}(\Gamma)$ defined as

$$
c_{0}(\Gamma):=\left\{x \in \mathbb{R}^{\Gamma} ; \forall \epsilon>0 \quad \#\{\gamma \in \Gamma ;|x(\gamma)|>\epsilon\}<\omega\right\}
$$

endowed with the supremum norm $\|x\|_{\infty}:=\sup \{|x(\gamma)| ; \gamma \in \Gamma\}, x \in c_{0}(\Gamma)$. Observe that every $x \in c_{0}(\Gamma)$ has a countable support.

We introduce the following norm on $c_{0}(\Gamma)$. Given $x \in c_{0}(\Gamma)$, put

$$
\|x\|:=\left(\sum_{n=1}^{\infty} 2^{-n} \| x\left\lceil_{\Gamma_{n}} \|_{\infty}^{2}\right)^{\frac{1}{2}}\right.
$$

where $\mathcal{G}:=\left(\Gamma_{n}\right)_{n \in \mathbb{N}}$ is a countable basis for the topology of $\Gamma$ such that $\Gamma_{1}:=\Gamma$.

We shall see first that $\|x \mid\|$ is a norm on $c_{0}(\Gamma)$. Obviously, for every $r \in \mathbb{R}$ we have $|\|r x|\|=|r| \cdot|\|x \mid\|$. We now show that $|\| x|\|$ is subadditive. To this end, observe that \|\|$x\|=\|\left(2^{-n / 2}\left\|x \Gamma_{\Gamma_{n}}\right\|_{\infty}\right) \|_{2}$, where $\|\cdot\|_{2}$ is the Hilbertian norm in $\ell_{2}(\mathbb{N})$. Then, 
given $x$ and $y$ in $c_{0}(\Gamma)$,

$$
\begin{aligned}
& \|\| x+y\|=\|\left(2^{-n / 2}\left\|(x+y)\left\lceil_{\Gamma_{n}} \|_{\infty}\right)\right\|_{2}\right. \\
& \quad \leq \|\left(2^{-n / 2}\left\|x\left\lceil_{\Gamma_{n}} \|_{\infty}\right)\right\|_{2}+\|\left(2^{-n / 2}\left\|y\left\lceil_{\Gamma_{n}} \|_{\infty}\right)\right\|_{2}\right.\right. \\
& \quad=\|x \mid\|+\|y\| .
\end{aligned}
$$

We will check next that $\|\cdot \mid\|$ is equivalent to $\|\cdot\|_{\infty}$. Clearly, for every $x \in c_{0}(\Gamma)$,

$$
\|x \mid\|^{2} \leq\|x\|_{\infty}^{2} \sum_{n=1}^{\infty} 2^{-n}=\|x\|_{\infty}^{2}
$$

hence $\|x\| \leq\|x\|_{\infty}$ for every $x \in c_{0}(\Gamma)$. On the other hand,

$$
\|x\|^{2} \geq 2^{-1}\left\|x \Gamma_{\Gamma_{1}}\right\|^{2}=2^{-1}\|x\|_{\infty}^{2}
$$

hence $\|x\|\left\|\geq 2^{-1 / 2}\right\| x \|_{\infty}$ for every $x \in c_{0}(\Gamma)$. This proves that $\|\cdot \mid\|$ is an equivalent norm.

We shall prove now that $\left|\|\cdot \mid\|\right.$ on $c_{0}(\Gamma)$ is strictly convex. Let us first recall the definition.

Definition 78 A norm $\|\cdot\|$ on a Banach space $X$ is called strictly convex (or rotund) if, whenever $x, y \in S_{X}$ satisfy $\|x+y\|=2$, then $x=y$.

Theorem 79 The norm $\|\cdot\| \mid \|$ defined on $c_{0}(\Gamma)$ by formula (3.1) is strictly convex.

Proof. Let $x, y \in c_{0}(\Gamma)$ be two distinct points with $\|x \mid\|=\|y\|=1$. Find then some $\gamma_{0} \in \Gamma$ such that $x\left(\gamma_{0}\right) \neq y\left(\gamma_{0}\right)$. We distinguish three cases.

CASE 1. Assume $y\left(\gamma_{0}\right)=0$. Then we have $\left|x\left(\gamma_{0}\right)\right|>\left|y\left(\gamma_{0}\right)\right|(=0)$. Let $F_{x}:=\{\gamma \in$ $\left.\Gamma ;|x(\gamma)| \geq\left|x\left(\gamma_{0}\right)\right|\right\}$. The set $F_{x}$ is finite, so we can find $n \in \mathbb{N}$ such $\gamma_{0} \in \Gamma_{n}$ and $\Gamma_{n} \cap F_{x}=\left\{\gamma_{0}\right\}$. Thus $\left\|x \Gamma_{\Gamma_{n}}\right\|_{\infty}=\left|x\left(\gamma_{0}\right)\right|$.

Put $F_{y}:=\left\{\gamma \in \Gamma ;|y(\gamma)| \geq(1 / 2)\left|x\left(\gamma_{0}\right)\right|\right\}$. The set $F_{y}$ is finite and, moreover, $\gamma_{0} \notin F_{y}$. Then, there exists $m \in \mathbb{N}$ such that $\gamma_{0} \in \Gamma_{m}$ and $\Gamma_{m} \cap F_{y}=\emptyset$. It follows that $\| y\left\lceil_{\Gamma_{m}} \| \leq(1 / 2)\left|x\left(\gamma_{0}\right)\right|<\left|x\left(\gamma_{0}\right)\right|\right.$.

By the fact that the family $\mathcal{G}$ is a basis for the topology of $\Gamma$, we can find $k \in \mathbb{N}$ such that $\gamma_{0} \in \Gamma_{k} \subset \Gamma_{n} \cap \Gamma_{m}$. Then

$$
\| y\left\lceil_{\Gamma_{k}}\left\|_{\infty}<\right\| x \Gamma_{\Gamma_{k}} \|_{\infty} .\right.
$$

CASE 2. Assume $0 \neq\left|y\left(\gamma_{0}\right)\right|<\left|x\left(\gamma_{0}\right)\right|$. Define $F_{x}:=\left\{\gamma \in \Gamma ;|x(\gamma)| \geq\left|y\left(\gamma_{0}\right)\right|\right\}$. The set $F_{x}$ is finite, so there exists $n \in \mathbb{N}$ such that $\Gamma_{n} \cap F_{x}=\left\{\gamma_{0}\right\}$. Therefore, $\left\|x\left|\Gamma_{n} \|_{\infty}=\right| x\left(\gamma_{0}\right) \mid\right.$. The set $F_{y}:=\left\{\gamma \in \Gamma ;|y(\gamma)| \geq\left|y\left(\gamma_{0}\right)\right|\right\}$ is also finite, so we can choose $m \in \mathbb{N}$ such that $\gamma_{0} \in \Gamma_{m}$ and $F_{y} \cap \Gamma_{m}=\left\{\gamma_{0}\right\}$. We obtain $\| y\left\lceil_{\Gamma_{m}} \|_{\infty}=\left|y\left(\gamma_{0}\right)\right|\right.$. As before, we can find $k \in \mathbb{N}$ such that $\gamma_{0} \in \Gamma_{k} \subset \Gamma_{n} \cap \Gamma_{m}$. Then

$$
\| y\left\lceil\Gamma _ { k } \| _ { \infty } = | y ( \gamma _ { 0 } ) | < | x ( \gamma _ { 0 } ) | = \| x \left\lceil_{\Gamma_{k}} \|_{\infty} .\right.\right.
$$


CASE 3. Finally, consider $x, y \in c_{0}(\Gamma)$ such that $\left|x\left(\gamma_{0}\right)\right|=\left|y\left(\gamma_{0}\right)\right|$. Observe that, in this case, we have $0 \neq x\left(\gamma_{0}\right)=-y\left(\gamma_{0}\right)$. Define $F_{x}:=\left\{\gamma \in \Gamma ;|x(\gamma)|>(1 / 3)\left|x\left(\gamma_{0}\right)\right|\right\}$, a finite set. Find again $n \in \mathbb{N}$ such that $\gamma_{0} \in \Gamma_{n}$ and $\Gamma_{n} \cap F_{x}=\left\{\gamma_{0}\right\}$. Then $\left\|x \Gamma_{\Gamma_{n}}\right\|=\left|x\left(\gamma_{0}\right)\right|$. Let $F_{y}:=\left\{\gamma \in \Gamma ;|y(\gamma)|>(1 / 3)\left|y\left(\gamma_{0}\right)\right|\right\}$, again a finite set. Find $m \in \mathbb{N}$ such that $\gamma_{0} \in \Gamma_{m}$ and $\Gamma_{m} \cap F_{y}=\left\{\gamma_{0}\right\}$. Then $\| y\left\lceil_{\Gamma_{m}} \|=\left|y\left(\gamma_{0}\right)\right|\right.$. There exists $k \in \mathbb{N}$ such that $\Gamma_{k} \subset \Gamma_{m} \cap \Gamma_{n}$. Then $\left\|x \Gamma_{\Gamma_{k}}\right\|_{\infty}=\| y\left\lceil_{\Gamma_{k}} \|_{\infty}\right.$. Since $(x+y)\left(\gamma_{0}\right)=0$, it follows that

$$
\|(x+y)\left\lceil_ { \Gamma _ { k } } \| _ { \infty } < \| x \left\lceil_ { \Gamma _ { k } } \| _ { \infty } + \| y \left\lceil_{\Gamma_{k}} \|_{\infty} .\right.\right.\right.
$$

For the cases 1 and 2, vectors given by

$$
\hat{x}=\left(2^{-n}\left\|\left.x\right|_{\Gamma_{n}}\right\|_{\infty}\right) \quad \text { and } \quad \hat{y}=\left(2^{-n}\left\|\left.y\right|_{\Gamma_{n}}\right\|_{\infty}\right)
$$

belong to $S_{\ell_{2}}$ and $\hat{x} \neq \hat{y}$. Since $\|\cdot\|_{\ell_{2}}$ is strictly convex, we have $\|\hat{x}+\hat{y}\|_{\ell_{2}}<$ $\|\hat{x}\|_{\ell_{2}}+\|\hat{y}\|_{\ell_{2}}$, i.e.,

$$
|\|x+y|\|<|\|x|\|+|\|y \mid\| .
$$

Finally, in the third case, since $\left\|\left.(x+y)\right|_{\Gamma_{n}}\right\| \leq\left\|\left.x\right|_{\Gamma_{n}}\right\|+\left\|\left.y\right|_{\Gamma_{n}}\right\|$ for all $n \in \mathbb{N}$, and, for $k$ as above, $\left\|\left.(x+y)\right|_{\Gamma_{k}}\right\|_{\infty}<\left\|\left.x\right|_{\Gamma_{k}}\right\|_{\infty}+\left\|\left.y\right|_{\Gamma_{k}}\right\|_{\infty}$, we get again

$$
\||| x+y|\|<|\|x|\|+|\|y \mid\| .
$$

This proves that the norm $|\|\cdot\|| \|$ is strictly convex. 



\section{Chapter 4}

\section{Some remarks on Krein's theorem}

This chapter deals with some aspects related to a quantification of Krein's theorem on the weak compactness of the closed convex hull of a weakly compact subset of a Banach space, introduced in [FHMZ]. This is a topic that only tangentially touches the subject of WCG Banach spaces and their relatives. The connection was presented in [FHMZ], and has to do with the characterization of subspaces of WCG obtained in [FMZ04]. Indeed, there was proved that a Banach space $X$ is a subspace of a $W C G$ Banach space $Z$ if (and only if) for every $\varepsilon>0$ its closed unit ball $B_{X}$ can be covered with a sequence $\left(A_{n}\right)$ of $\varepsilon$-weakly compact subsets of $X$. Here, a set $A \subset X$ is called $\varepsilon$-weakly compact if it is bounded and $\bar{A}^{w^{*}} \subset X+\varepsilon B_{X^{* *}}$. It was natural to ask whether the sets $A_{n}$ in the above characterization could be taken to be convex. This was the motivation for the study of a sort of quantified Krein's theorem.

Here we investigate some aspect of this theory and we precise some result from [FHMZ], in many cases by using different techniques, like the concept of upper envelopes of continuous linear functions acting on balls.

\subsection{Upper envelopes and distances from points in $X^{* *}$ to $X$}

In this chapter, and until it will be said explicitly, all functions considered will have domain $B_{X^{*}}$, where $X$ is a given Banach space.

In [DGZ, Section III.2], the concept of concave $w^{*}$-upper semicontinuous envelope of an element $x^{* *}$ in the bidual space $X^{* *}$ was introduced in order to deal with the concept of roughness and of octahedrality.

Our purpose is to treat some aspects of a quantitative version of Krein's theorem. We propose then the following extension of the former concept. Let $M$ be a non-empty 
subset of a Banach space $X$ - that will remain fixed in the rest of the section. We consider on the dual unit ball $B_{X^{*}}$ the topology $w\left(X^{*}, M\right)$ of the pointwise convergence on the set $M$. This topology is not necessarily Hausdorff. In fact, if it were Hausdorff, it would coincide with the $w^{*}$-topology on $B_{X^{*}}$, as it is coarser than the $w^{*}$-topology.

Definition 80 Let $X$ be a Banach space. Given $x^{* *} \in X^{* *}$, the concave $w\left(X^{*}, M\right)$ usc envelope of $x^{* *}$ is a function defined on $B_{X^{*}}$ by

$$
\hat{x}_{M}^{* *}:=\inf \left\{f ; f: B_{X^{*}} \rightarrow \mathbb{R} ; f \text { is } w\left(X^{*}, M\right) \text {-continuous and } f \geq x^{* *}{ }_{\mid B_{X^{*}}}\right\} .
$$

When $M:=X$ we recover the concept of of concave $w^{*}$-upper semicontinuous envelope of an element $x^{* *} \in X^{* *}$ used in [DGZ, Section III.2].

Given a function $f: B_{X^{*}} \rightarrow \mathbb{R}$, its hypograph is the set hgraph $(f):=\left\{\left(x^{*}, t\right) \in B_{X^{*}} \times\right.$ $\left.\mathbb{R} ; t \leq f\left(x^{*}\right)\right\}$. We endow the set $X^{*} \times \mathbb{R}$ with the product topology $w\left(X^{*}, M\right) \times R$, where $R$ denotes the usual topology on $\mathbb{R}$. The following result characterizes $\hat{x}_{M}^{* *}$ by describing its hypograph.

Proposition 81 Let $x^{* *} \in X^{* *}$. Then

$$
\operatorname{hgraph}\left(\hat{x}_{M}^{* *}\right)={\overline{\operatorname{hgraph}\left(x^{* *}\right)}}^{w\left(X^{*}, M\right) \times R} .
$$

Proof. First of all, it is clear that $\hat{x}_{M}^{* *} \geq x^{* *}$. Thus, $\operatorname{hgraph}\left(x^{* *}\right) \subset \operatorname{hgraph}\left(\hat{x}_{M}^{* *}\right)$. Assume that ${\overline{\operatorname{hgraph}\left(x^{* *}\right)}}^{w\left(X^{*}, M\right) \times R} \neq \operatorname{hgraph}\left(\hat{x}_{M}^{* *}\right)$. We can find then $x^{*} \in B_{X^{*}}$ such that $\left(x^{*} ; \hat{x}_{M}^{* *}\left(x^{*}\right)\right) \notin \overline{\operatorname{hgraph}\left(x^{* *}\right)} w\left(X^{*}, M\right) \times R$. Obviously, the set $\overline{\operatorname{hgraph}\left(x^{* *}\right)} w\left(X^{*}, M\right) \times R$ is convex and $w\left(X^{*}, M\right) \times R$-closed. Then we can find $x \in$ $M$ and $\alpha \in \mathbb{R}$ such that the graph of $x+\alpha$ separates $\overline{\operatorname{hgraph}\left(x^{* *}\right)} w\left(X^{*}, M\right) \times R$ and $\left(x^{*}, \hat{x}_{M}^{* *}\left(x^{*}\right)\right)$. In particular, because $x^{* *} \leqslant\langle x, \cdot\rangle+\alpha$ and so $\hat{x}_{M}^{* *} \leqslant\left\langle x, x^{*}\right\rangle+\alpha$ for each $x^{*} \in B_{X^{*}}$, we have

$$
\hat{x}_{M}^{* *}\left(x^{*}\right)>\left\langle x, x^{*}\right\rangle+\alpha \geqslant \hat{x}_{M}^{* *}\left(x^{*}\right),
$$

a contradiction. This proves that

$$
\operatorname{hgraph}\left(\hat{x}_{M}^{* *}\right) \subseteq \overline{\operatorname{hgraph}\left(x^{* *}\right)} w\left(X^{*}, M\right) \times R .
$$

On the other hand, $\hat{x}_{M}^{* *}$ is $w\left(X^{*}, M\right)$-usc by the very definition (see Corollary 83 ). This proves that $\operatorname{hgraph}\left(\hat{x}_{M}^{* *}\right)$ is $w\left(X^{*}, M\right) \times R$-closed and we get the conclusion.

Remark 82 It follows from the proof of Proposition 81 that $\hat{x}_{M}^{* *}$ can be described by using fewer $w\left(X^{*}, M\right)$-continuous functions, namely

$$
\hat{x}_{M}^{* *}\left(x^{*}\right)=\inf \left\{\left\langle x, x^{*}\right\rangle+\lambda ; x \in M, \lambda \in \mathbb{R} \text { such that } x+\lambda \geqslant x^{* *} \text { on } B_{X^{*}}\right\}
$$

for all $x^{*} \in B_{X^{*}}$. Indeed, in the separation of a point $\left(x^{*}, t\right)$ and the set $\overline{\operatorname{hgraph}\left(x^{* *}\right)} w\left(X^{*}, M\right) \times R$, we use a function $x+\lambda$, where $x \in M$ and $\lambda \in \mathbb{R}$. 
Corollary 83 Given $x^{* *} \in X^{* *}$, the function $\hat{x}_{M}^{* *}$ is concave and $w\left(X^{*}, M\right)$-usc.

Proof. In the proof of Proposition 81 we showed that $\operatorname{hgraph}\left(\hat{x}_{M}^{* *}\right)$ is convex. This proves the first part. The second assertion is a consequence of the definition. Indeed, the infimum of a family of $w\left(X^{*}, M\right)$-continuous functions is $w\left(X^{*}, M\right)$-usc. This justifies the last part of the proof of Proposition 81.

The following proposition gives an alternative description of $\hat{x}_{M}^{* *}$.

Proposition 84 Let $X$ be a Banach space. Then, given $x^{* *} \in X^{* *}$,

$$
\hat{x}_{M}^{* *}\left(x^{*}\right)=\lim _{N \in \mathcal{N}_{M}\left(x^{*}\right)} \sup \left\langle x^{* *}, N\right\rangle, \quad \text { for every } \quad x^{*} \in B_{X^{*}},
$$

where $\mathcal{N}_{M}\left(x^{*}\right)$ denotes the family of all neighborhoods of $x^{*}$ in $\left(B_{X^{*}}, w\left(B_{X^{*}}, M\right)\right)$.

Proof. Fix $x^{*} \in B_{X^{*}}$. Let $\left(x_{\iota}^{*}\right)$ be a net in $B_{X^{*}}$ that $w\left(B_{X^{*}}, M\right)$-converges to $x^{*}$ such that, for some $t_{\iota} \leqslant\left\langle x^{* *}, x_{\iota}^{*}\right\rangle$, we have

$$
\left(x_{i}^{*}, t_{i}\right) \rightarrow\left(x^{*}, \hat{x}_{M}^{* *}\left(x^{*}\right)\right) .
$$

This can be done in view of Proposition 81. Fix $N \in \mathcal{N}_{M}\left(x^{*}\right)$. We can find $\iota_{0}$ such that, for all $\iota \geqslant \iota_{0}, x_{\iota}^{*} \in N$. Then we have

$$
t_{\iota} \leqslant\left\langle x^{* *}, x_{\iota}^{*}\right\rangle \leqslant \sup \left\langle x^{* *}, N\right\rangle .
$$

It follows from (4.1) that $\hat{x}_{M}^{* *}\left(x^{*}\right) \leqslant \sup \left\langle x^{* *}, N\right\rangle$. This happens for every $N \in \mathcal{N}_{M}\left(x^{*}\right)$. Hence

$$
\hat{x}_{M}^{* *}\left(x^{*}\right) \leqslant \lim _{N \in \mathcal{N}_{M}\left(x^{*}\right)} \sup \left\langle x^{* *}, N\right\rangle .
$$

Assume now that $\hat{x}_{M}^{* *}\left(x^{*}\right)<\lim _{N \in \mathcal{N}_{M}\left(x^{*}\right)} \sup \left\langle x^{* *}, N\right\rangle$. Find $\alpha \in \mathbb{R}$ such that $\hat{x}_{M}^{* *}\left(x^{*}\right)<$

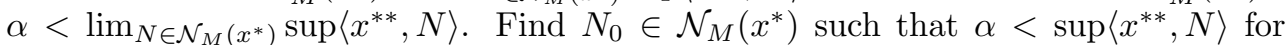
all $N \in \mathcal{N}_{M}\left(x^{*}\right)$ such that $N \subset N_{0}$. (Indeed, observe that $N \mapsto \sup \left\langle x^{* *}, N\right\rangle$ is an "increasing" function.) This means, in particular, that we can find $x_{N}^{*} \in N$ such that $\alpha<\sup \left\langle x^{* *}, x_{N}^{*}\right\rangle$, for all $N \in \mathcal{N}_{M}\left(x^{*}\right)$ such that $N \subset N_{0}$. Obviously, $\left\langle x^{* *}, x_{N}^{*}\right\rangle \leqslant\left\|x^{* *}\right\|$ for all $N \in \mathcal{N}_{M}\left(x^{*}\right)$ with $N \subset N_{0}$. So we may assume, without loss of generality, that $\left\langle x^{* *}, x_{N}^{*}\right\rangle \rightarrow l$, for some $l(\geqslant \alpha)$ in $\mathbb{R}$. Then we have

$$
\left(x_{N}^{*},\left\langle x^{* *}, x_{N}^{*}\right\rangle\right) \rightarrow w\left(X^{*}, M\right) \times R\left(x^{*}, l\right),
$$

where $R$ denotes the usual topology in $\mathbb{R}$. This proves that

$$
\left(x^{*}, l\right) \in{\overline{\operatorname{hgraph}\left(x^{* *}\right)}}^{w\left(X^{*}, M\right) \times R}\left(=\operatorname{hgraph}\left(\hat{x}_{M}^{* *}\right)\right) .
$$

In particular, $\alpha \leqslant l \leqslant \hat{x}_{M}^{* *}\left(x^{*}\right)$, a contradiction. 
Proposition 85 Let $X$ be a Banach space. Then, given $x^{* *} \in X^{* *}$,

$$
\hat{x}_{M}^{* *}\left(x^{*}\right)=\inf \left\{\left\langle x, x^{*}\right\rangle+\left\|x^{* *}-x\right\| ; x \in M\right\} \quad \text { for every } x^{*} \in B_{X^{*}} .
$$

Proof. The function $x^{* *}$ is obviously bounded on $B_{X^{*}}$. Let $\lambda_{0} \in \mathbb{R}$ and $x_{0} \in M$ be such that $\left\langle x_{0}, x^{*}\right\rangle+\lambda_{0} \geqslant x^{* *}\left(x^{*}\right)$, for each $x^{*} \in B_{X^{*}}$. Then $\left\langle x^{* *}-x_{0}, x^{*}\right\rangle \leqslant \lambda_{0}$ for all $x^{*} \in B_{X^{*}}$. In particular, $\left\|x^{* *}-x_{0}\right\| \leqslant \lambda_{0}$. We get

$$
\left\langle x_{0}, x^{*}\right\rangle+\lambda_{0} \geqslant\left\langle x_{0}, x^{*}\right\rangle+\left\|x^{* *}-x_{0}\right\| \text { for every } x^{*} \in B_{X^{*}} .
$$

We take infima at the right side on $x \in M$,

$$
\left\langle x_{0}, x^{*}\right\rangle+\lambda_{0} \geqslant \inf \left\{\left\langle x, x^{*}\right\rangle+\left\|x^{* *}-x\right\| ; x \in M\right\} .
$$

This happens for every $x_{0} \in M$ and every $\lambda \in \mathbb{R}$ such that $x_{0}+\lambda \geqslant x^{* *}$ on $B_{X^{*}}$. Because of Remark 82, we get

$$
\hat{x}_{M}^{* *}\left(x^{*}\right) \geqslant \inf \left\{\left\langle x, x^{*}\right\rangle+\left\|x^{*}-x\right\| ; x \in M\right\} .
$$

On the other side,

$$
\left\langle x^{* *}-x, x^{*}\right\rangle \leqslant\left\|x-x^{* *}\right\|, \quad \forall x^{*} \in B_{X^{*}}, \quad \forall x \in M .
$$

Then $\left\langle x^{* *}, x^{*}\right\rangle \leqslant\left\langle x, x^{*}\right\rangle+\left\|x-x^{* *}\right\|$, for every $x^{*} \in B_{X^{*}}$, and for every $x \in M$. It follows that

$$
\hat{x}_{M}^{* *}\left(x^{*}\right) \leqslant\left\langle x, x^{*}\right\rangle+\left\|x-x^{* *}\right\| \quad \forall x^{*} \in B_{X^{*}}, \quad \forall x \in M .
$$

Therefore

$$
\hat{x}_{M}^{* *}\left(x^{*}\right) \leqslant \inf \left\{\left\langle x, x^{*}\right\rangle+\left\|x-x^{* *}\right\| ; x \in M\right\}, \quad \forall x^{*} \in B_{X^{*}} .
$$

This proves the reverse inequality.

Remark 86 Let $x^{* *} \in X^{* *}$. From Proposition 85, it follows that $\hat{x}_{M}^{* *}(0)=\inf \{\langle x, 0\rangle+$ $\left.\left\|x-x^{* *}\right\| ; x \in M\right\}$, hence $\hat{x}_{M}^{* *}(0)=\operatorname{dist}\left(x^{* *}, M\right)(=: d)$, where dist $\left(x^{* *}, M\right)$ denotes the distance from $x^{* *}$ to $M$ in the norm $\|\cdot\|$ on $X^{* *}$. From Proposition 84 we have that, for every $N \in \mathcal{N}_{M}(0), d \leqslant \sup \left\langle x^{* *}, N\right\rangle$, and for every $\varepsilon>0$, there exists $N_{\varepsilon} \in \mathcal{N}_{M}(0)$ such that $\sup \left\langle x^{* *}, N_{\varepsilon}\right\rangle<d+\varepsilon$. This will be used later (in particular, in Proposition 96).

\subsection{Quantitative Krein's Theorem}

\subsubsection{Interchanging limits and $\varepsilon$-WSK sets}

The following two definitions were introduced in [FHMZ]. The first one was inspired by the concept of interchanging limits, due to Grothendieck [Gr52], and the second is a quantification of the usual weak relative compactness, and was motivated by the characterization of subspaces of weakly compactly generated Banach spaces done in [FMZ04]. 
Definition 87 Let $M$ be a bounded subset of a Banach space $X$, and let $S$ be a bounded subset of $X^{*}$. We say that $M \varepsilon$-interchanges limits with $S$, and in this case we write $M \S \varepsilon \oint S$, if for any two sequences $\left(x_{n}\right)$ in $M$ and $\left(x_{m}^{*}\right)$ in $S$ such that the following limits exist,

$$
\lim _{n \rightarrow \infty} \lim _{m \rightarrow \infty}\left\langle x_{n}, x_{m}^{*}\right\rangle, \quad \lim _{m \rightarrow \infty} \lim _{n \rightarrow \infty}\left\langle x_{n}, x_{m}^{*}\right\rangle
$$

then

$$
\left|\lim _{n \rightarrow \infty} \lim _{m \rightarrow \infty}\left\langle x_{n}, x_{m}^{*}\right\rangle-\lim _{m \rightarrow \infty} \lim _{n \rightarrow \infty}\left\langle x_{n}, x_{m}^{*}\right\rangle\right| \leqslant \varepsilon .
$$

Definition 88 Given $\varepsilon \geq 0$, a subset $M$ of a Banach space is said to be $\varepsilon$-weakly compact $(\varepsilon-W K)$ if it is bounded and $\bar{M}^{w^{*}} \subset X+\varepsilon B_{X^{* *}}$.

Obviously, if a bounded set $M \subset X$ is $\varepsilon$-WK, we have dist $\left(x^{* *}, X\right) \leq \varepsilon$ for every $x^{* *} \in \bar{M}^{w^{*}}$, and this distance is realized. We shall show that there are Banach spaces $(X,\|\cdot\|)$ and bounded sets $M \subset X$ such that $\operatorname{dist}\left(x^{* *}, X\right) \leq \varepsilon$ for every $x^{* *} \in \bar{M}^{w^{*}}$ and yet $M$ is not $\varepsilon-\mathrm{WK}$. To this end, it is worth to observe the proof of [FHMZ, Propositions 8(i) and 14(i)]. Following the argument there, we see that something more precise that statements (i) in both propositions is, in fact, proved. We record it for future reference and we omit the proof, since it is, word by word, the one provided there.

Proposition 89 Let $(X,\|\cdot\|)$ be a Banach space. Let $M \subset X$ be a bounded subset such that $\operatorname{dist}\left(x^{* *}, X\right) \leq \varepsilon$ for every $x^{* *} \in \bar{M}^{w^{*}}$. Then, $M \S 2 \varepsilon \S B_{X^{*}}$, and $M \S \varepsilon \S\left\{x_{1}^{*}, x_{2}^{*}, \ldots\right\}$ for every $w^{*}$-null sequence $\left(x_{n}^{*}\right)$ in $B_{X^{*}}$.

Example 90 There exits a Banach space $(X,\|\cdot\|)$ and a bounded set $M \subset X$ such that $\operatorname{dist}\left(x^{* *}, X\right) \leq \varepsilon$ for every $x^{* *} \in \bar{M}^{w^{*}}$ and yet $M$ is not $\varepsilon-W K$.

Proof. Let $(X,\|\cdot\|)$ be the Banach space $c_{0}$ endowed with the following equivalent norm:

$$
\|x\|:=\|x\|_{\infty}+\sum_{i=1}^{\infty} \frac{1}{2^{i}}\left|x_{i}\right|, \quad x:=\left(x_{i}\right) \in c_{0},
$$

where $\|\cdot\|_{\infty}$ is the canonical supremum norm. It is simple to prove that the bidual norm in $X^{* *}:=\ell_{\infty}$ is given by

$$
\left\|x^{* *}\right\|:=\left\|x^{* *}\right\|_{\infty}+\sum_{i=1}^{\infty} \frac{1}{2^{i}}\left|x_{i}^{* *}\right|, \quad x^{* *}:=\left(x_{i}^{* *}\right) \in \ell_{\infty},
$$

where, again, $\|\cdot\|_{\infty}$ is the canonical supremum norm. All the distances below will be computed in the $\|\cdot\|$-norm. Let $\mathbb{I}:=(1,1, \ldots) \in \ell_{\infty}$, and $\mathbb{I}_{n}:=\sum_{i=1}^{n} e_{i} \in c_{0}$, where $e_{i}$ is the $i$ th vector of the canonical basis of $c_{0}$. Let $M:=\left\{\mathbb{I}_{n} ; n \in \mathbb{N}\right\}$; this is a bounded subset of $c_{0}$. Then, obviously, $\bar{M}^{w^{*}}=M \cup\{\mathbb{I}\}$. Clearly, $\operatorname{dist}(\mathbb{I}, X) \geq 1$, 
and $\left\|\mathbb{I}-\mathbb{I}_{n}\right\| \rightarrow 1$ as $n \rightarrow \infty$. Then, $\operatorname{dist}(\mathbb{I}, X)=1$. From Proposition 89 we get that $M \S 1 \S B_{(X,\|\cdot\|)}$. We shall prove now that there is no element $x \in X$ such that $\|\mathbb{I}-x\|=1$. Indeed, let $x \in X$. Then, $\|\mathbb{I}-x\|_{\infty} \geq 1$. Assume that $\|\mathbb{I}-x\|=1$. Then $\sum_{i=1}^{\infty} \frac{1}{2^{i}}\left|x_{i}\right|=0$, and this implies $x=0$. However, $\|\mathbb{I}-0\|=2$, and we reach a contradiction.

In order to precise a behavior investigated in [FHMZ] and [FMZ07], we introduce here a definition. It gives a name to the property of a bounded subset of a Banach space whose $w^{*}$-closure in $X^{* *}$ is not far from itself.

Definition 91 Let $M$ be a bounded subset of a Banach space. Let $\varepsilon \geq 0$. The set $M$ is $\varepsilon$-weakly self-compact $(\varepsilon-W S K)$ if $\bar{M}^{w^{*}} \subset \bar{M}^{\|\cdot\|}+\varepsilon B_{X^{* * *}}$.

There is a connection between the concept of being $\varepsilon$-WK and of being $\varepsilon$-WSK, at least in case of bounded closed and convex sets. It is plain that, in general, $\varepsilon$-WSK implies $\varepsilon$-WK. In [FMZ04], the following lemma appears. We shall reproduce here its proof to underline that the method cannot give Proposition 96 below.

Lemma 92 Let $(X,\|\cdot\|)$ be a Banach space, let $C \subset X$ be a non-empty convex set, and let $x^{* *} \in \bar{C}^{w^{*}}$. Then

$$
\operatorname{dist}\left(x^{* *}, C\right) \leq 2 \operatorname{dist}\left(x^{* *}, X\right)
$$

Proof. Take any $\delta$ such that $\operatorname{dist}\left(x^{* *}, X\right)<\delta$ and find $x \in X$ such that $\left\|x^{* *}-x\right\|<\delta$. Then $x \in \bar{C}^{w^{*}}+\delta B_{X^{* *}} \subset \overline{C+\delta B_{X}} w^{*}$. It follows that $x \in \overline{C+\delta B_{X}}\|\cdot\|$. Therefore, given $\varepsilon>0$ there exists $c \in C$ and $b \in B_{X}$ such that $\|x-c-\delta b\|<\varepsilon$; so $\|x-c\| \leq \varepsilon+\delta$. Finally we get $\left\|x^{* *}-c\right\|=\left\|x^{* *}-x+x-c\right\| \leq 2 \delta+\varepsilon$, and then $\operatorname{dist}\left(x^{* *}, C\right) \leq 2 \delta+\varepsilon$. As $\varepsilon>0$ was arbitrary, $\operatorname{dist}\left(x^{* *}, C\right) \leq 2 \delta$. Therefore $\operatorname{dist}\left(x^{* *}, C\right) \leq 2 \operatorname{dist}\left(x^{* *}, X\right)$.

As a result, the following holds.

Corollary 93 Let $M$ be a bounded closed convex subset of a Banach space $X$ and let $\varepsilon \geq 0$. Then, if $M$ is $\varepsilon-W K$ then it is $(2 \varepsilon+\delta)-W S K$ for every $\delta>0$.

Proof. Let $x^{* *} \in \bar{M}^{w^{*}}$. Then dist $\left(x^{* *}, X\right) \leq \varepsilon$. From Lemma 92 it follows that $\operatorname{dist}\left(x^{* *}, M\right) \leq 2 \operatorname{dist}\left(x^{* *}, X\right) \leq 2 \varepsilon$. Then, given $\delta>0$, there exists $x \in M$ such that $\left\|x^{* *}-x\right\|<2 \varepsilon+\delta$. We can conclude then that $\bar{M}^{w^{*}} \subset M+(2 \varepsilon+\delta) B_{X^{* *}}$.

Lemma 92 obviously does not hold in general for non-convex sets. A simple example is given by the subset $\lambda S_{X}$ of a Banach space $X$, where $\lambda>0$. The origin belongs to the weak closure of $\lambda S_{X}$; we have dist $\left(0, \lambda S_{X}\right)=\lambda$ and dist $(0, X)=0$. Neither Corollary 93 holds in general for non-convex sets. Again, a simple example is given by the set $M:=\left\{x_{1}, x_{2}, \ldots\right\} \subset c_{0}$, where $x_{n}:=(1,1, \ldots, k, 0,0, \ldots)$ and $k$ is some fixed number greater than 1 in the $n$th position, $n \in \mathbb{N}$. Let $e:=(1,1, \ldots) \in \ell_{\infty}$. Then $x_{n} \stackrel{w^{*}}{\rightarrow} e$, so $\bar{M} w^{w^{*}}=M \cup\{e\}$. Clearly, dist $\left(e, c_{0}\right)=1$, so the set $M$ is $1-\mathrm{WK}$. 
However, $\left\|e-x_{n}\right\|=k-1$ for every $n \in \mathbb{N}$. The set $M$ is closed in $c_{0}$. We obtain, finally, that $\bar{M}^{w^{*}}$ is not included in $\bar{M}^{\|\cdot\|}+(k-1-\delta) B_{\ell_{\infty}}$ for every $\delta>0$ such that $k-1-\delta>0$. In particular, take $k:=3+2 \delta$ for any $\delta>0$.

In $[\mathrm{FHMZ}]$ the following proposition was proved.

Proposition 94 ([FHMZ]) Let $M$ be a bounded subset of a Banach space $X$, and $\varepsilon \geqslant 0$ some number. Then we have

(i) If $M$ is $\varepsilon-W K$, then $M \S 2 \varepsilon \S B_{X^{*}}$.

(ii) If $M \S \varepsilon \S B_{X^{*}}$, then $M$ is $\varepsilon-W K$.

We claim that there is a small gap in the statement of (ii) in Proposition 94. It is not a problem for the quantitative treatment of the weak compactness done there, but it should be clarified. The small problem concerns the absence of weak compactness in the set of points in $X$ close enough to some $x^{* *} \in X^{* *}$, and the statement in (ii) should read as follows:

(ii') If $M \S \varepsilon \S B_{X^{*}}$ then $M$ is $(\varepsilon+\delta)-W K$ for every $\delta>0$.

The validity of the statement (ii') is clear. Indeed, from the proof of Proposition 8 in [FHMZ] it follows that, if $M$ is a bounded subset of a Banach space $X$ such that $M \S \varepsilon \S B_{X^{*}}$, and $z \in \bar{M}^{w^{*}}$, then $\operatorname{dist}(z, X) \leq \varepsilon$. So, given $\delta>0$ there exists $x \in X$ such that $\|z-x\|<\varepsilon+\delta$. Then, $\bar{M}^{w^{*}} \subset X+(\varepsilon+\delta) B_{X^{* *}}$, so $M$ is $(\varepsilon+\delta)-\mathrm{WK}$, as claimed.

The following result appears in [FHMZ] as a certain variation of the result quoted above as Proposition 94.

Proposition 95 ([FHMZ], Proposition 14) Let $M$ be a bounded subset of a Banach space $X$, and let $\varepsilon>0$. Then

(i) If $M$ is $\varepsilon-W K$ then $M \oint \varepsilon \oint\left\{x_{1}^{*}, x_{2}^{*}, \ldots\right\}$, where $\left(x_{n}^{*}\right)$ is any $w^{*}$-null sequence in $B_{X^{*}}$. (ii) If $\left(B_{X^{*}}, \omega^{*}\right)$ is angelic and $M \oint \varepsilon \oint\left\{x_{1}^{*}, x_{2}^{*}, \ldots\right\}$ for any $w^{*}$-null sequence $\left(x_{n}^{*}\right)$ in $B_{X^{*}}$, then $M$ is $\varepsilon-W K$.

We may again object to the second statement. From the very proof of this proposition in $[\mathrm{FHMZ}$ ] we can conclude that only the following was proved.

(ii') If $\left(B_{X^{*}}, \omega^{*}\right)$ is angelic and $M \S \varepsilon \oint\left\{x_{1}^{*}, x_{2}^{*}, \ldots\right\}$ for any $w^{*}$-null sequence $\left(x_{n}^{*}\right)$ in $B_{X^{*}}$, then $M$ is $(\varepsilon+\delta)-W K$ for every $\delta>0$.

Indeed, it is proved there that, if $M \oint \varepsilon \oint\left\{x_{1}^{*}, x_{2}^{*}, \ldots\right\}$ for any $w^{*}$-null sequence $\left(x_{n}^{*}\right)$ in $B_{X^{*}}$, then $\operatorname{dist}\left(x_{0}^{* *}, X\right)=\varepsilon$, for every $x_{0}^{* *} \in \bar{M}^{w^{*}}$, and the conclusion follows as before.

This worse estimate is not just a byproduct of the proof done in [FHMZ, Proposition 14]. That some small adjustment should be done is clear, since we can provide a 
counterexample to the statement (ii) in Proposition 95. In order to show it, we rely on Example 90 and on the notation introduced there. The space $(X,\|\cdot\|)$ is just $c_{0}$ with the norm defined in (4.2). This space is separable, so $\left(B_{X^{*}}, w^{*}\right)$ is angelic (in fact, it is a compact metrizable space). It follows from the fact that $\operatorname{dist}\left(x^{* *}, X\right) \leq 1$ for all $x^{* *} \in \bar{M}^{w^{*}}$ and Proposition 89 that $M \S 1 \S\left\{x_{1}^{*}, x_{2}^{*}, \ldots\right\}$ for every $w^{*}$-null sequence $\left(x_{n}^{*}\right)$ in $B_{X^{*}}$. However, we showed in Example 90 that the set $M$ is not 1-WK in the sense of Definition 88.

Returning to the general case, it follows from Proposition 94 (ii') and from Corollary 93 that every closed convex and bounded subset $M$ of a Banach space $X$ such that $M \S \varepsilon \S B_{X^{*}}$ is $(2 \varepsilon+\delta)-W S K$, for every $\delta>0$. We shall prove something better; indeed, $M$ is then $(\varepsilon+\delta)$-WSK, for every $\delta>0$, even in case that $M$ is not necessarily convex and/or closed.

Proposition 96 Let $M$ be a bounded set in a Banach space $X$, and let $\varepsilon \geqslant 0$. If $M \S \varepsilon \S B_{X^{*}}$ then $M$ is $(\varepsilon+\delta)$-WSK for every $\delta>0$.

Proof. Assume that $M \S \varepsilon \S B_{X^{*}}$. Let $x^{* *} \in \bar{M}^{w^{*}}$ and denote $d:=d\left(x^{* *}, M\right)$. We shall define inductively two sequences, $\left(x_{n}\right)$ in $M$ and $\left(x_{m}^{*}\right)$ in $B_{X^{*}}$. To begin with, choose any $x_{1} \in M$. Define then $N_{1}:=\left\{x^{*} \in B_{X^{*}} ;\left|\left\langle x_{1}, x^{*}\right\rangle\right|<1\right\}$; this is a neighbourhood of 0 in $\left(B_{X^{*}}, w\left(X^{*}, M\right)\right)$. By Remark 86, we can find $x_{1}^{*} \in N_{1}$ such that

$$
d-1 \leqslant\left\langle x^{* *}, x_{1}^{*}\right\rangle<d+1 .
$$

Choose $x_{2} \in M$ such that $\left|\left\langle x^{* *}-x_{2}, x_{1}^{*}\right\rangle\right|<\frac{1}{2}$. Let us define $N_{2}:=\left\{x^{*} \in B_{X^{*}}\right.$; $\left.\left|\left\langle x_{i}, x^{*}\right\rangle\right|<\frac{1}{2}, i=1,2\right\}$; this is an element of $\mathcal{N}_{M}(0)$. Pick then $x_{2}^{*} \in N_{2}$ such that $d-\frac{1}{2} \leqslant\left\langle x^{* *}, x_{2}^{*}\right\rangle<d+1 / 2$. Continue in this way. We get sequences $\left(x_{n}\right)$ and $\left(x_{m}^{*}\right)$ such that

$$
\begin{gathered}
x_{n} \in M, \quad x_{m}^{*} \in B_{X^{*}}, \quad \forall n, m, \\
\left|\left\langle x^{* *}-x_{n}, x_{m}^{*}\right\rangle\right|<\frac{1}{n}, \quad m=1,2, \ldots, n-1, \\
\left|\left\langle x_{n}, x_{m}^{*}\right\rangle\right|<\frac{1}{m}, \quad n=1,2, \ldots, m, \\
d-\frac{1}{m} \leqslant\left\langle x^{* *}, x_{m}^{*}\right\rangle<d+\frac{1}{m}, \quad m=1,2, \ldots
\end{gathered}
$$

Then

$$
\begin{gathered}
\lim _{n}\left\langle x_{n}, x_{m}^{*}\right\rangle=\left\langle x^{* *}, x_{m}^{*}\right\rangle, \quad \forall m, \\
\lim _{m} \lim _{n}\left\langle x_{n}, x_{m}^{*}\right\rangle=\lim \left\langle x^{* *}, x_{m}^{*}\right\rangle=d, \\
\lim _{m}\left\langle x_{n}, x_{m}^{*}\right\rangle=0, \quad \forall n, \\
\lim _{n} \lim _{m}\left\langle x_{n}, x_{m}^{*}\right\rangle=0,
\end{gathered}
$$

so

$$
\left|\lim _{m} \lim _{n}\left\langle x_{n}, x_{m}^{*}\right\rangle-\lim _{n} \lim _{m}\left\langle x_{n}, x_{m}^{*}\right\rangle\right|=d \leqslant \varepsilon
$$


Theorem 97 Let $(X,\|\cdot\|)$ be a Banach space. Let $M$ be a bounded subset of $X$. Assume that $\bar{M}^{w^{*}} \subset M+\varepsilon B_{X^{* *}}$ for some $\varepsilon \geqslant 0$. Then $\overline{\operatorname{conv}(M)} w^{*} \subset \operatorname{conv}(M)+$ $(2 \varepsilon+\delta) B_{X^{* *}}$ for every $\delta>0$.

Proof. Fix $\delta>0$. From Proposition 94(i), we have $M \S 2 \varepsilon \S B_{X^{*}}$. Now, by [FHMZ, Theorem 13] we get $\operatorname{conv}(M) \S 2 \varepsilon \S B_{X^{*}}$. Therefore, from Proposition 96, we have $\overline{\operatorname{conv}(M)} w^{*} \subset \operatorname{conv}(M)+(2 \varepsilon+\delta) B_{X^{* *}}$.

\subsection{2 $\varepsilon$-WSK sets in natural settings}

We shall justify that the concept introduced in Definition 91 naturally appears in the study of some geometric and functional-analytic properties of Banach spaces. We just collect some definitions and results showing that this is the case. We refer to the literature for proofs.

Let $M$ be a bounded subset of a Banach space $(X,\|\cdot\|)$. Let

$$
\left\|x^{*}\right\|_{M}:=\sup \left\{\left\langle x, x^{*}\right\rangle ; x \in M\right\}, \quad x^{*} \in X^{*} .
$$

The function $\|\cdot\|_{M}$ defined on $X^{*}$ is then a seminorm. Concepts like $\operatorname{dist}_{M}$ or $\operatorname{diam}_{M}$ are defined with respect to this seminorm. For example, given $x^{*}, y^{*} \in X^{*}$, $\operatorname{dist}_{M}\left(x^{*}, y^{*}\right)=\left\|x^{*}-y^{*}\right\|_{M}$, and so on.

Let us introduce the following definition.

Definition 98 Let $M$ be a bounded subset of a Banach space $(X,\|\cdot\|)$. We say that the norm $\|\cdot\|$ is $M$-uniformly Gâteaux smooth if $\lim _{n \rightarrow \infty}\left\|f_{n}-g_{n}\right\|_{M}=0$ whenever $f_{n}, g_{n} \in S_{X^{*}}, n \in \mathbb{N}$, are such that $\lim _{n \rightarrow \infty}\left\|f_{n}+g_{n}\right\|=2$.

Obviously, if $M:=B_{X}$, we are in the case of a uniformly Gâteaux smooth norm. In order to characterize Banach spaces having an equivalent $M$-uniformly Gâteaux smooth norm, in [FMZ], the following process was defined.

By a $w^{*}$-slice of a set $D \subset X^{*}$ we understand the intersection of $D$ with a $w^{*}$-open halfspace in $X^{*}$. Given a bounded set $M \subset X, \varepsilon>0$, and $D \subset B_{X^{*}}$, we introduce the $(M, \varepsilon)$-dentability derivative of $D$ by

$$
D_{(M, \varepsilon)}^{\prime}=\left\{f \in D ; \operatorname{diam}_{M}(S) \geq \varepsilon \text { for each } w^{*} \text {-slice } S \text { of } D \text { containing } f\right\}
$$

Let $\alpha>1$ be an ordinal number and assume that we already defined a dentability derivatve $D_{(M, \varepsilon)}^{(\beta)}$ for every ordinal $\beta<\alpha$. If $\alpha-1$ exists, we define the $\alpha$-th $(M, \varepsilon)$ dentability derivative of $D$ as $D_{(M, \varepsilon)}^{(\alpha)}=\left(D_{(M, \varepsilon)}^{(\alpha-1)}\right)_{(M, \varepsilon)}^{\prime}$. Otherwise, we put $D_{(M, \varepsilon)}^{(\alpha)}=$ $\bigcap_{\beta<\alpha} D_{(M, \varepsilon)}^{(\beta)}$. We observe a simple fact that, if $D$ is convex and $w^{*}$-closed, then so is $D_{(M, \varepsilon)}^{\prime}$. 
Definition 99 Let $(X,\|\cdot\|)$ be a Banach space. Let a bounded set $M \subset X$ and $\varepsilon>0$ be given. We say that $M$ has finite (resp. countable) $\varepsilon$-dual index if $\left(B_{X^{*}}\right)_{(M, \varepsilon)}^{(\alpha)}=$ $\emptyset$ for some finite (resp. countable) ordinal number $\alpha$. The first ordinal with this property, if it exists, is called the $\varepsilon$-dual index of $M$.

One of the main results in [FMZ] is the following.

Theorem 100 ([FMZ], Theorem 5) Let $(X,\|\cdot\|)$ be a Banach space. Let $M$ be a bounded subset of $X$. Then the following assertions are equivalent:

(i) $X$ admits an equivalent $M$-uniformly Gâteaux smooth norm.

(ii) $M$ has a finite $\varepsilon$-dual index for every $\varepsilon>0$.

It was also proved in [FMZ] that sets with finite $\varepsilon$-dual index belong to the class introduced in Definition 91. More precisely, the following result holds.

Theorem 101 ([FMZ], Theorem 10) Let $M$ be a bounded closed convex subset of a Banach space $(X,\|\cdot\|)$, and $\varepsilon>0$ be given.

If $M$ has finite $\varepsilon$-dual index, then, for every $\varepsilon^{\prime}>\varepsilon, M$ is $2 \varepsilon^{\prime}-W S K$.

In particular, if $M$ has finite $\varepsilon$-dual index for every $\varepsilon>0$, then $M$ is weakly compact. 


\section{Chapter 5}

\section{Flat sets, $\ell_{p}$-generating and fixing $c_{0}$ in nonseparable setting}

\subsection{Introduction}

In [GKL00] it was proved that a separable Banach space $X$ is isomorphic to a subspace of $c_{0}$ if and only if it has an equivalent C-Lipschitz $w^{*}$-Kadec-Klee (in short, $C$ $\left.L K K^{*}\right)$ norm for some $C \in(0,1]$. The norm $\|\cdot\|$ on $X$ is said to be $C$-LKK* whenever $\lim \sup _{n \rightarrow \infty}\left\|x^{*}+x_{n}^{*}\right\| \geq\left\|x^{*}\right\|+C \lim \sup _{n \rightarrow \infty}\left\|x_{n}^{*}\right\|$ for every $x^{*} \in X^{*}$ and every $w^{*}$-null sequence $\left(x_{n}^{*}\right)$ in $X^{*}$. The norm is called $\mathrm{LKK}^{*}$ if it is $C$-LKK ${ }^{*}$ for some $C \in(0,1]$. The supremum norm on $c_{0}$ is $1-\mathrm{LKK}^{*}$ (see a more general result in Proposition 135).

We say that Banach space $(X,\|\cdot\|)$ has the Kadec-Klee property (KK, in short) if, for every $x \in X$ and every weakly null sequence $\left(x_{n}\right)$ in $X$ such that $\left\|x+x_{n}\right\| \rightarrow\|x\|$, we have $\left\|x_{n}\right\| \rightarrow 0$. We say that $(X,\|\cdot\|)$ has the Kadec-Klee* property $\left(\mathrm{KK}^{*}\right.$, in short) if the dual norm has the property that, for every $x^{*} \in X^{*}$ and every $w^{*}$-null sequence $\left(x_{n}^{*}\right)$ in $X^{*}$ such that $\left\|x^{*}+x_{n}^{*}\right\| \rightarrow\left\|x^{*}\right\|$, we have $\left\|x_{n}^{*}\right\| \rightarrow 0$. Finally, we say that $X$ has the weak-Kadec-Klee* property $\left(w-\mathrm{KK}^{*}\right.$, in short) if the dual norm has the property that, for every $x^{*} \in X^{*}$ and every $w^{*}$-null sequence $\left(x_{n}^{*}\right)$ in $X^{*}$ such that $\left\|x^{*}+x_{n}^{*}\right\| \rightarrow\left\|x^{*}\right\|$, we have $x_{n}^{*} \stackrel{w}{\rightarrow} 0$. There is a discrepancy between these definitions and others provided in some of the literature available. For example, in [FHHMPZ01, Exercise 8.83], it is said that $X^{*}$ has the property $w^{*}-\mathrm{KK}$ if the norm and $w^{*}$-topologies coincide on the dual unit sphere $S_{X^{*}}$. It is customary to reserve the letter K (from Kadec) for the coincidence of the topologies, while KK (for KadecKlee) is reserved for the coincidence of convergent (with respect to both topologies) sequences in the unit sphere on the dual space. In this Memoir we shall stick to this 
last terminology.

The name C-Lipschitz $w^{*}$-Kadec-Klee for the property of the norm $\|\cdot\|$ in $X$ described above is justified by the fact that it implies the $\mathrm{KK}^{*}$ property of the space. This will be explicitly stated in Proposition 117.

V. D. Milman introduced, in [M71], the following modulus. If $X$ is a Banach space, $x \in S_{X}, Y$ is a linear subspace of $X$ and $\tau>0$,

$$
\bar{\rho}(\tau, x, Y):=\sup \{\|x+y\|-1 ; y \in Y,\|y\| \leq \tau\}
$$

then

$$
\bar{\rho}(\tau, x):=\inf \{\bar{\rho}(\tau, x, Y) ; \operatorname{dim}(X / Y)<\infty\}
$$

and, finally,

$$
\bar{\rho}(\tau):=\sup \left\{\bar{\rho}(\tau, x) ; x \in S_{X}\right\} .
$$

It turns out that the norm $\|\cdot\|$ on $X$ is $L K K^{*}$ if and only if there exists $\tau_{0}>0$ such that $\bar{\rho}\left(\tau_{0}\right)=0$, and it is $1-L K K^{*}$ if and only if $\bar{\rho}(1)=0$ (for details and more on the subject see [GKL00], where a non-separable theory is also developed). The geometric description provided by the use of the modulus $\bar{\rho}$ is more clear than the one given by the definition of a $C$-LKK*-norm above, and can be depicted as $B_{X}$ being asymptotically uniformly flat. Accordingly, a separable Banach space is $\mathrm{LKK}^{*}$ if and only if $B_{X}$ is asymptotically uniformly flat, and this characterizes the class of spaces isomorphic to subspaces of $c_{0}$. The last affirmation is the main result in [GKL00], as we mentioned at the beginning of this Introduction.

In this chapter, we shall use ideas from [GKL00] to deal with $\ell_{p}\left(\omega_{1}\right)$-generation of Banach spaces for $p \in(1,+\infty]$, and operators fixing copies of $c_{0}\left(\omega_{1}\right)$. We work in the context of nonseparable weakly compactly generated (WCG) spaces (see Remark 152). The restriction of the density to the first uncountable cardinal is done for the sake of simplicity. It is plausible that our results work with less cardinal restrictions.

If $M$ is a bounded set in a Banach space $X$, we shall denote by $\|\cdot\|_{M}$ the seminorm defined on $X^{*}$ by

$$
\left\|x^{*}\right\|_{M}=\sup \left\{\left|\left\langle x, x^{*}\right\rangle\right| ; x \in M\right\}, \quad x^{*} \in X^{*} .
$$

This function was already introduced in the paragraph 4.2.2, equation (4.3).

Notation. As we mentioned in the Introduction, the first infinite ordinal and the first uncountable ordinal are denoted by $\omega_{0}$ and $\omega_{1}$, and their cardinalities by $\aleph_{0}$ and $\aleph_{1}$, respectively. We identify an ordinal number $\Omega$ with the interval $[0, \Omega)$. Throughout this chapter we assume that $\frac{+\infty}{+\infty}=1$ and that $\frac{1}{0}=+\infty$. 


\section{$5.2 \quad$ Asymptotically $p$-flat and $p$-flat sets}

\subsubsection{Asymptotically $p$-flat sets}

The following concept evolves from the definition of $C$-LKK* given above. It will be used in characterizing WCG Asplund spaces that are generated by $c_{0}\left(\omega_{1}\right)$ or by $\ell_{p}\left(\omega_{1}\right)$ for $p \in(1,+\infty)$ (see Theorem 156).

Definition $102 \operatorname{Let}(X,\|\cdot\|)$ be a Banach space $X$, let $M \subset X$ be a set, let $p \in$ $(1,+\infty]$, and let $q:=\frac{p}{p-1}$ be its conjugate index. We say that $M$ is $\|\cdot\|$-asymptotically $p$-flat if it is bounded and there exists $C>0$ such that, for every $f \in X^{*}$ and every $w^{*}$-null sequence $\left(f_{n}\right)$ in $X^{*}$, we have

$$
\limsup _{n \rightarrow \infty}\left\|f+f_{n}\right\|^{q} \geq\|f\|^{q}+C \limsup _{n \rightarrow \infty}\left\|f_{n}\right\|_{M}^{q} .
$$

We say that $M$ is asymptotically $p$-flat if there exists an equivalent norm $\|\cdot \mid\|$ on $X$ such that $M$ is $|\|\cdot \mid\|$-asymptotically $p$-flat.

According to the notation introduced above, the case $\|\cdot\|$-asymptotically $\infty$-flat corresponds to the use of the exponent $q=1$ in formula (5.5).

\subsubsection{Basic results and some remarks}

We shall make some remarks and provide some results about the concept introduced in Definition 102

Remark 103 Given a Banach space $(X,\|\cdot\|)$, the $w^{*}$-lower semicontinuity of the dual norm gives that, for every $w^{*}$-null sequence $\left(f_{n}\right)$ in $X^{*}$ and for every $f \in X^{*}$, we always have, for $q \in[1,+\infty)$, $\lim _{\sup _{n \rightarrow \infty}}\left\|f+f_{n}\right\|^{q} \geq\|f\|^{q}$. Equation (5.5) quantifies a more precise behavior of $\left(f_{n}\right)$ with respect to some bounded subset $M$ of $X$.

Remark 104 Definition 102 uses $w^{*}$-null sequences in the dual of a general Banach space $X$. The origin in $X^{*}$ belongs to the $w^{*}$-closure of $S_{X^{*}}$. If $X$ is separable, as in the context of [GKL00], the metrizability of $\left(B_{X^{*}}, w^{*}\right)$ ensures that there exists a $w^{*}$-null sequence in $S_{X^{*}}$. If, on the contrary, $X$ is not separable, the existence of such a sequence is guaranteed by the deep theorem of Josefson and Niszenweig (see, for example, [Di84, Chapter XII]). It is possible, then, to check the $\|\cdot\|$-asymptotically $p$-flatness of a certain bounded subset $M$ of $X$ without risking that the very concept should be trivial in the sense of absence of $w^{*}$-null sequences in $X^{*}$ that were not already $\|\cdot\|$-null.

Remark 105 Definition 102 restricts the range of exponents to $(1, \infty]$. The reason for that lies in Theorem 156. Indeed, we shall see there that the existence of a linearly dense and $\|\cdot\|$-asymptotically $p$-flat subset, with $p \in(1,+\infty)$ (resp., $p=+\infty)$ of an Asplund Banach space $X$ is equivalent to the existence of a linear and bounded 
operator from $\ell_{p}\left(\omega_{1}\right)$ (resp. $c_{0}\left(\omega_{1}\right)$ ) into $X$ with dense range. Now, "most" nonseparable Banach spaces admit a continuous linear injection from $\ell_{1}(\Gamma)$ into them, as shown in Remark 153. Our goal is to describe the "non-trivial" situation, hence we shall focus on $p>1$ only.

It is also worth to mention from the beginning that our interest is mainly in the non-separable setting, in view of Remark 152.

We shall start with some results about the concept of asymptotically $p$-flatness. The first one is just a trivial observation that follows from the very definition, so we shall omit its proof. It is, however, worth to mention, in view of the opposite situation described in Proposition 148.

Proposition 106 Let $(X,\|\cdot\|)$ be a Banach space. Then, for every $p \in(1, \infty]$, the absolutely convex closed hull of any $\|\cdot\|$-asymptotically $p$-flat subset of $X$, with constant $C>0$, is again $\|\cdot\|$-asymptotically $p$-flat, with the same constant $C$.

The next result allows us to simplify some computations just by looking at elements in the dual unit sphere.

Proposition 107 In the definition of $\|\cdot\|$-asymptotically p-flatness (Definition 102) it is possible to substitute the expression "for every $f \in X^{*}$ " by the expression "for every $f \in S_{\left(X^{*},\|\cdot\|\right)}$ ", with no change in the concept being defined.

Proof. Let us call " $\|\cdot\|-(D) "$ Definition 102 in the norm $\|\cdot\|$, while " $\|\cdot\|$-(DS)" denotes the corresponding definition using elements $f \in S_{X^{*}}$. Obviously, $\|\cdot\|-(\mathrm{D}) \Rightarrow\|\cdot\|-(\mathrm{DS})$. Assume, now, that a set $M \subset X$ satisfies $\|\cdot\|$-(DS). Obviously, in the inequality (5.5) we may change, without loss of generality, the constant $C$ to another positive constant (called again $C$ ) strictly less that 1 . Since $M$ is bounded, we can find $\lambda>0$ such that $\lambda M \subset(1 / C) B_{(X,\|\cdot\|)}$. Consider any $w^{*}$-null sequence $\left(f_{n}\right)$ in $X^{*}$. Let $f:=0$. We shall prove that $\limsup _{n \rightarrow \infty}\left\|f_{n}\right\|^{q} \geq C \limsup _{n \rightarrow \infty}\left\|f_{n}\right\|_{\lambda M}^{q}$, where $\left(f_{n}\right)$ is a $w^{*}$ null sequence in $X^{*}$. Indeed, we have $\limsup _{n \rightarrow \infty}\left\|f_{n}\right\|^{q} \geq \limsup _{n \rightarrow \infty}\left\|f_{n}\right\|_{\lambda M C}^{q}=$ $(\lambda C)^{q} \lim \sup _{n \rightarrow \infty}\left\|f_{n}\right\|_{M}^{q}$.

Assume now $0 \neq f \in X^{*}$. Then

$$
\limsup _{n \rightarrow \infty}\left\|\frac{f}{\|f\|}+\frac{f_{n}}{\|f\|}\right\|^{q} \geq 1+C \limsup _{n \rightarrow \infty}\left\|\frac{f_{n}}{\|f\|}\right\|_{M}^{q},
$$

and then

$$
\limsup _{n \rightarrow \infty}\left\|f+f_{n}\right\|^{q} \geq\|f\|^{q}+C \limsup _{n \rightarrow \infty}\left\|f_{n}\right\|_{M}^{q} .
$$

Now, let $C^{\prime}:=\min \left\{C,(\lambda C)^{q}\right\}(>0)$. Then we get, for all $f \in X^{*}$,

$$
\limsup _{n \rightarrow \infty}\left\|f+f_{n}\right\|^{q} \geq\|f\|^{q}+C^{\prime} \limsup _{n \rightarrow \infty}\left\|f_{n}\right\|_{M}^{q} \text {. }
$$

This proves the result. 
Remark 108 Let $(X,\|\cdot\|)$ be a Banach space. Assume that $B_{X}$ is $\|\cdot\|$-asymptotically $p$-flat for some $p \in(1, \infty]$, and let $C$ be the constant in (5.5). If $X$ is infinitedimensional, there exists, by the Josefson-Niszenweig theorem (see, e.g., [Di84, Chapter XII]), a $w^{*}$-null sequence $\left(f_{n}\right)$ in $S_{X^{*}}$. Take $f \in S_{X^{*}}$. Then,

$$
2^{q} \geq \limsup _{n \rightarrow \infty}\left\|f+f_{n}\right\|^{q} \geq\|f\|^{q}+C \limsup _{n}\left\|f_{n}\right\|^{q}=1+C .
$$

It follows that $C \leq 2^{q}-1$. In particular, if $B_{X}$ is $\|\cdot\|$-asymptotically $\infty$-flat, then $C \leq 1$.

Remark 109 Obviously, from the very definition of asymptotically $p$-flatness, the constant $C$ in (5.5) can be replaced by a smaller one without changing the norm. More precisely, if the inequality (5.5) holds for some $C>0$, for every $f \in X^{*}$ and every $w^{*}$-null sequence $\left(f_{n}\right)$ in $X^{*}$, it also holds for every $0<C^{\prime} \leq C$, again for every $f \in X^{*}$ and every $w^{*}$-null sequence $\left(f_{n}\right)$ in $X^{*}$. This should be compared with the statement in the simple Proposition 110. From this proposition it follows that we can also raise the constant without affecting the whole situation - although now we must change (easily) the norm. The constant $C$ becomes, then, irrelevant. It appears only as a matter of control in the computations needed.

Proposition 110 Let $(X,\|\cdot\|)$ be a Banach space. Let $M \subset X$ be a $\|\cdot\|$-asymptotically $p$-flat set, for some $p \in(1, \infty]$ and some constant $C>0$ as in (5.5). Then, for every $C^{\prime}>0$ there exists an equivalent norm $\||\cdot|\|$ on $X$ such that $M$ is $\|\cdot|\||$-asymptotically $p$-flat with constant $C^{\prime}$.

Proof. Let $K>0$. Put $\|x\|:=(1 / K)\|x\|, x \in X$. The function $\|\cdot \mid\|$ is obviously an equivalent norm on $X$ and $\|\cdot\|=(1 / K)\|\cdot\| \|$ in $X^{*}$. Then, for $f \in X^{*}$ and a $w^{*}$-null sequence $\left(f_{n}\right)$ in $X^{*}$,

$$
\limsup _{n \rightarrow \infty}\left\|f+f_{n}\right\|^{q} \geq\|f\|^{q}+C \limsup _{n \rightarrow \infty}\left\|f_{n}\right\|_{M}^{q},
$$

i.e.,

$$
K^{q} \limsup _{n \rightarrow \infty}\left\|f+f_{n}\right\|^{q} \geq K^{q}\|f\|^{q}+K^{q} C \limsup _{n \rightarrow \infty}\left\|f_{n}\right\|_{M}^{q},
$$

so we have

$$
\limsup _{n \rightarrow \infty}\left|\left\|f+f_{n}\left|\left\|^{q} \geq\left|\|f\|^{q}+K^{q} C \limsup _{n \rightarrow \infty}\right|\right\| f_{n} \|_{M}^{q} .\right.\right.\right.
$$

Since $K>0$ is arbitrary, so it is $C^{\prime}:=K^{q} C$. We get that $M$ is $\||\|\mid\|$-asymptotically $p$-flat with constant $C^{\prime}$.

Remark 111 The concept of asymptotically $p$-flatness is defined in an isomorphic way, avoiding the intrinsic isometric character of the concept of $\|\cdot\|$-asymptotically $p$-flatness (formula (5.5)). To see that this happens (i.e., that a bounded set can be $\|\cdot\|$-asymptotically $p$-flat but not $|\| \cdot|||$-asymptotically $p$-flat for another equivalent norm $\|\cdot \mid\|$ on $X$ ), consider the following simple proposition and the Example 113. 
Proposition 112 Let $(X,\|\cdot\|)$ be a Banach space with some point $x_{0} \in S_{X}$ where the norm is LUR. Then, $B_{X}$ is not $\|\cdot\|$-asymptotically $\infty$-flat (i.e., the Banach space is not $\mathrm{LKK}^{*}$ ).

Proof. From the LUR property of $\|\cdot\|$ at $x_{0}$, there exists a function $\delta\left(x_{0}, \cdot\right)$ : $(0,2] \rightarrow \mathbb{R}^{+}$such that, given $\varepsilon \in(0,2], z \in X$ with $\|z\| \geq \varepsilon$ and $\left\|x_{0}+z\right\| \leq 1$, then $\left\|x_{0}+(z / 2)\right\| \leq 1-\delta\left(x_{0}, \varepsilon\right)$. Obviously,

$$
2 x_{0}=\left(x_{0}-\frac{z}{2}\right)+\left(x_{0}+\frac{z}{2}\right),
$$

so

$$
2 \leq\left\|x_{0}-\frac{z}{2}\right\|+\left\|x_{0}+\frac{z}{2}\right\|
$$

Therefore,

$$
\left\|x_{0}+\frac{z}{2}\right\| \geq 2-\left\|x_{0}-\frac{z}{2}\right\| \geq 2-\left(1-\delta\left(x_{0}, \varepsilon\right)\right)=1+\delta\left(x_{0}, \varepsilon\right) .
$$

Since $\|(z / 2)\|=\varepsilon / 2$, it follows that, according (5.1), $\bar{\rho}\left(\varepsilon / 2, x_{0}, Y\right) \geq \delta\left(x_{0}, \varepsilon\right)$ for every linear subspace $Y$ of $X$. Then, from (5.2), we have $\bar{\rho}\left(\varepsilon / 2, x_{0}\right) \geq \delta\left(x_{0}, \varepsilon\right)$. Following (5.3), we get $\bar{\rho}(\varepsilon / 2) \geq \delta\left(x_{0}, \varepsilon\right)$. If we use now the result of Milman mentioned in the Introduction to this chapter, we get that the space $X$ is not $\mathrm{LKK}^{*}$, i.e., $B_{(X,\|\cdot\|)}$ is not asymptotically $\infty$-flat in the norm $\|\cdot\|$.

We can now provide the sought example to see that being $\|\cdot\|$-asymptotically $p$-flat (at least for the $\infty$-flatness and in the separable case) really depends on the norm $\|\cdot\|$ of the Banach space.

Example 113 There exists a Banach space $(X,\|\cdot\|)$ and a $\|\cdot\|$-asymptotically $p$-flat subset $M$ which is not $|\|\cdot \mid\|$-asymptotically - -flat in some equivalent norm $\|\cdot \mid\|$.

Proof From Proposition 135 below, $B_{\left(c_{0},\|\cdot\|_{\infty}\right)}$ is $\|\cdot\|_{\infty}$-asymptotically $\infty$-flat. The space $c_{0}$ has an equivalent LUR norm \|\|$\cdot\|\|$ (Day's norm is such, see, e.g., [Di75, Chapter 4]). We may assume that $B_{\left(c_{0},\|\cdot\| \cdot \|\right)} \subset B_{\left(c_{0},\|\cdot\|_{\infty}\right)}$. From Proposition 106, it follows that $B_{\left(c_{0},\|\cdot\| \cdot \|\right)}$ is $\|\cdot\|_{\infty}$-asymptotically $\infty$-flat. However, $B_{\left(c_{0},\|\cdot\| \cdot \|\right)}$ is not $\|||||$-asymptotically $\infty$-flat, thanks to Proposition 112.

The following two propositions show some natural classes of asymptotically $p$-flat sets in Banach spaces. Although the second class (limited sets) is more general than the first one (\| $\cdot \|$-compact sets), we prefer to start by this particular instance, since $\|\cdot\|$-compactness is somehow more natural. The proof of the second proposition is identical with the proof of the first one, so we shall skip it.

Proposition 114 Let $(X,\|\cdot\|)$ be a Banach space. Then, every $\|\cdot\|$-compact set $M$ in $X$ is $\|\cdot\|$-asymptotically $p$-flat for all $p \in(1, \infty]$. 
Proof. Take any $\varepsilon>0$. Find a finite set $F$ in $X$ so that $M \subset F+\varepsilon B_{X}$. Let $\left(f_{n}\right)$ be a sequence as in Definition 102. Then $\lim _{n \rightarrow \infty}\left\|f_{n}\right\|_{F}=0$, and so $\limsup _{n \rightarrow \infty}\left\|f_{n}\right\|_{M} \leq$ $\varepsilon \cdot \sup _{n \in \mathbb{N}}\left\|f_{n}\right\|$. Hence, as $\varepsilon>0$ was arbitrary, we conclude that $\lim _{n \rightarrow \infty}\left\|f_{n}\right\|_{M}=0$, and (5.5) is trivially satisfied.

Recall that a set $M$ in a Banach space $X$ is limited if $\lim _{n \rightarrow \infty}\left\|f_{n}\right\|_{M}=0$ whenever $\left(f_{n}\right)$ is a $w^{*}$-null sequence in $X^{*}$. Then, almost the same proof of Proposition 114 gives the following result.

Proposition 115 Let $(X,\|\cdot\|)$ be a Banach space. Then, every limited set in $X$ is $\|\cdot\|$-asymptotically $p$-flat for all $p \in[1, \infty)$.

Remark 116 1. Not every asymptotically $p$-flat set is limited: $B_{c_{0}}$ is $\|\cdot\|_{\infty^{-}}$ asymptotically $\infty$-flat in $c_{0}$ (see Proposition 135 ), although it contains the nonlimited set $\left\{e_{n} ; n \in \mathbb{N}\right\}$. It is worth to recall here that a Banach space $(X,\|\cdot\|)$ with a limited unit ball is finite-dimensional. This is a non-trivial extension (it uses the deep Josefson-Nissenzweig theorem, see, e.g., [Di84]) of the elementary fact that the norm compactness of the unit ball forces the space to be finitedimensional.

2. Of course, the property of a set to be limited depends only on the dual pair. Then, if a set $M$ is limited in a Banach space, it is $\||\cdot| \mid$-asymptotically $p$-flat for every $p \in(1, \infty]$ and for every equivalent norm $\||\cdot|\|$ on $X$ (the constant $C>0$ can be also arbitrarily small).

The following result justifies, somehow, the name LKK* used in describing what we called asymptotically $\infty$-flat, since it follows that every Banach space with a closed unit ball asymptotically $\infty$-flat has the $\mathrm{KK}^{*}$ property. Notice that this applies not only to the case $p=\infty$, but also to any $p \in(1,+\infty]$. It is worth also to have in mind Proposition 118, since it proves that, necessarily, the context for the Godefroy, Lancien, and Kalton results mentioned in the Introduction is the class of separable Asplund spaces. In our more general setting, where $B_{X}$ is asymptotically $p$-flat (see Theorem 156), we are again, thanks to Proposition 118, dealing with Asplund spaces. However, in Theorem 158, and working with the more general situation (the existence of linearly dense and bounded innerly asymptotically $p$-flat sets), we shall avoid the Asplund setting.

Proposition $117 \operatorname{Let}(X,\|\cdot\|)$ be a Banach space. Assume that, for some $p \in(1, \infty]$, $B_{X}$ is a $\|\cdot\|$-asymptotically $p$-flat set. Then $(X,\|\cdot\|)$ has the $\mathrm{KK}^{*}$ property.

Proof. Fix $f \in S_{X^{*}}$ and a sequence $\left(g_{n}\right)$ in $S_{X^{*}}$ such that $g_{n} \stackrel{w^{*}}{\longrightarrow} f$. Let $f_{n}:=g_{n}-f$ for all $n \in \mathbb{N}$. Then, from Definition 102, we have, for some $C>0$,

$$
\begin{aligned}
1= & \limsup _{n \rightarrow \infty}\left\|g_{n}\right\|^{q}=\limsup _{n \rightarrow \infty}\left\|f+f_{n}\right\|^{q} \\
& \geq\|f\|^{q}+C \limsup _{n \rightarrow \infty}\left\|f_{n}\right\|^{q}=1+C \underset{n \rightarrow \infty}{\limsup }\left\|g_{n}-f\right\|^{q} .
\end{aligned}
$$


Then, $\lim \sup _{n \rightarrow \infty}\left\|g_{n}-f\right\|=0$, so $g_{n} \stackrel{\|\cdot\|}{\longrightarrow} f$.

A subset $A$ of a topological space $T$ is said to be angelic if every relatively countably compact subset $K$ of $A$ is relatively compact and, moreover, every point of $\bar{A}$ is the limit of a sequence from $A$. For example, every Eberlein compact space is angelic; so it is, in particular, the unit dual ball of a weakly compactly generated Banach space, in its $w^{*}$-topology (see, e.g., [Flo80]).

Proposition 118 Let $(X,\|\cdot\|)$ be a Banach space. Assume that $X$ has the $w-K K^{*}$ property and that $\left(B_{X^{*}}, w^{*}\right)$ is angelic. Then $X$ is Asplund.

Proof. The case when the Banach space $X$ is separable and has the property $w$-KK* is in [FHHMPZ01, Exercise 8.87]. The idea of the proof works too for the more general case we state here, and it is so neat that we cannot help but reproducing it, adapted to our situation. First of all, choose a countable dense subset $N:=\left\{x_{n} ; n \in \mathbb{N}\right\}$ of $S_{X}$. For each $n \in \mathbb{N}$ select an element $x_{n}^{*} \in S_{X^{*}}$ such that $\left\langle x_{n}, x_{n}^{*}\right\rangle=1$.

We claim that $C:=\bar{\Gamma}^{w^{*}}\left\{x_{n}^{*} ; n \in \mathbb{N}\right\}=B_{X^{*}}$. Indeed, if not, we can apply the Separation Theorem to the locally convex space $\left(X^{*}, w^{*}\right)$, to the compact convex set $C$, and to some point $x^{*} \in B_{X^{*}} \backslash C$. We can find then an $x \in S_{X}$ such that $\sup \langle x, C\rangle\left\langle\left\langle x, x^{*}\right\rangle(\leq 1)\right.$. Since $N$ is dense in $S_{X}$ we can find $n \in \mathbb{N}$ such that $(1 \leq) \sup \left\langle x_{n}, C\right\rangle<1$, a contradiction. This proves the claim.

Take now $x^{*} \in S_{X^{*}}$. The fact that $\left(B_{X^{*}}, w^{*}\right)$ is metrizable ensures the existence of a sequence $\left(c_{n}^{*}\right)$ in $C$ such that $c_{n}^{*} \stackrel{w^{*}}{\longrightarrow} x^{*}$. By the $w^{*}$-lower semicontinuity of the dual norm $\|\cdot\|$, we have $\lim _{n \rightarrow \infty}\left\|c_{n}^{*}\right\|=1$. It is enough now to use the $w$-KK* property to conclude that $c_{n}^{*} \stackrel{w}{\longrightarrow} x^{*}$. This proves that, in fact, $B_{X^{*}}=\bar{\Gamma}^{w}\left\{x_{n}^{*} ; n \in \mathbb{N}\right\} \quad(=$ $\left.\bar{\Gamma}^{\|\cdot\|}\left\{x_{n}^{*} ; n \in \mathbb{N}\right\}\right)$, and so $X^{*}$ is separable.

The non-separable case is not treated in [FHHMPZ01]. We provide here the proof. Let $Y$ be a separable subspace of $X$. Let $Q: X^{*} \rightarrow Y^{*}$ be the canonical quotient mapping. Let $\left(y_{n}^{*}\right)$ be a sequence in $S_{Y^{*}}$ that $w^{*}$-converges to some $y^{*} \in S_{Y^{*}}$. Select an arbitrary subsequence $\left(y_{n_{k}}^{*}\right)$ of $\left(y_{n}^{*}\right)$. Choose $x_{k}^{*} \in S_{X^{*}}$ such that $Q x_{k}^{*}=y_{n_{k}}^{*}$, $k \in \mathbb{N}$, and let $x^{*}\left(\in B_{X^{*}}\right)$ be a $w^{*}$-cluster point of $\left(x_{k}^{*}\right)$. Since $\left(B_{X^{*}}, w^{*}\right)$ is angelic we can find a further subsequence (denoted again $\left(x_{k}^{*}\right)$ ) that $w^{*}$-converges to $x^{*}$. Since $Q x^{*}=y^{*}\left(\in S_{Y^{*}}\right)$ we get $\left\|x^{*}\right\|=1$. Apply the fact that $X$ has the $w$-KK ${ }^{*}$ property to conclude that $x_{k}^{*} \rightarrow x^{*}$ in the weak topology. Therefore, $y_{n_{k}}^{*} \rightarrow y^{*}$ in the weak topology. The space $Y$ has then the $w$-KK* property. From the first part of the proof, $Y^{*}$ is separable. This proves that $X$ is Asplund.

Remark 119 A closer look at the proof of Proposition 118 shows that $w^{*}$-sequentially compactness of the closed dual unit ball of $X$, endowed with the $w^{*}$-topology (a weaker assumption than angelicity), was the only requirement needed. This applies, too, to Corollary 169 and Proposition 172.

The following is a renorming result. 
Proposition 120 Let $(X,\|\cdot\|)$ be a Banach space, let $p \in(1,+\infty]$, and let $M \subset X$ be a $\|\cdot\|$-asymptotically $p$-flat set. Let $\|\cdot\| \|$ be an equivalent $K K^{*}$ norm in $X$. Then there exists an equivalent $K K^{*}$ norm $|\cdot|$ on $X$ such that $M$ is $|\cdot|$-asymptotically $p$-flat. If $\||\cdot|||$ is an equivalent $K^{*}$ norm, the norm $|\cdot|$ can be taken to be also $K^{*}$.

Proof. Let $q=\frac{p}{p-1}$ (if $p=\infty$ then $q=1$ ), and define

$$
\left|x^{*}\right|^{q}:=\left\|x^{*}\right\|^{q}+\left\|\mid x^{*}\right\|^{q}, \quad x^{*} \in X^{*} .
$$

The function $|\cdot|$ so defined is an equivalent dual norm on $X^{*}$. To prove that the predual norm $|\cdot|$ is $\mathrm{KK}^{*}$, let $f \in S_{\left(X^{*},|\cdot|\right)}$ and $\left(f_{n}\right)$ be a sequence in $S_{\left(X^{*},|\cdot|\right)}$ such that $f_{n} \stackrel{w^{*}}{\longrightarrow} f$. Choose an arbitrary subsequence $\left(g_{n}\right)$ of $\left(f_{n}\right)$. By passing to a subsequence if necessary, we may assume that $\left\|g_{n}\right\|$ and $\left|\left\|g_{n} \mid\right\|\right.$ both converge. Let $l_{2}:=\lim _{n \rightarrow \infty}\left\|g_{n}\right\|$ and $l_{3}:=\lim _{n \rightarrow \infty}\left\|g_{n}\right\|$. Then, by the $w^{*}$-lower semicontinuity of the dual norm, $\|f\| \leq l_{2}$ and $\||f|\| \leq l_{3}$. Since $|f|^{q}=\|f\|^{q}+\|\| f \|^{q}=1$, we get $\left\|\left|\|f\|=l_{3}=\lim _{n \rightarrow \infty}\left\|g_{n} \mid\right\|\right.\right.$. By the $\mathrm{KK}^{*}$-property of \|\|$\cdot \|$ we get $g_{n} \stackrel{\|\cdot \mid\| \|}{\longrightarrow} f$, i.e., $g_{n} \stackrel{\|\cdot\|}{\longrightarrow} f$. Since this happens for a subsequence of an arbitrary subsequence of $\left(f_{n}\right)$ we get the conclusion.

Let $C>0$ be such that $\lim \sup _{n \rightarrow \infty}\left\|x^{*}+x_{n}^{*}\right\|^{q} \geq\left\|x^{*}\right\|^{q}+C \limsup _{n \rightarrow \infty}\left\|x_{n}^{*}\right\|_{M}^{q}$ for every $x^{*} \in X^{*}$ and every $w^{*}$-null sequence $\left(x_{n}^{*}\right)$ in $X^{*}$. Fix $x^{*} \in X^{*}$ and a $w^{*}$-null sequence $\left(x_{n}^{*}\right)$ in $X^{*}$. We can find a subsequence $\left(y_{n}^{*}\right)$ of $\left(x_{n}^{*}\right)$ such that the sequence $\left(\left\|x^{*}+y_{n}^{*}\right\|\right)$ converges to $\lim \sup _{n \rightarrow \infty}\left\|x^{*}+x_{n}^{*}\right\|$ and the sequence $\left(\left\|x^{*}+y_{n}^{*} \mid\right\|\right)$ converges (so $\left(\left|x^{*}+y_{n}^{*}\right|\right)$ converges, too). Then

$$
\begin{aligned}
& \limsup _{n \rightarrow \infty}\left|x^{*}+x_{n}^{*}\right|^{q} \geq \lim _{n \rightarrow \infty}\left|x^{*}+y_{n}^{*}\right|^{q} \\
& \quad=\limsup _{n \rightarrow \infty}\left\|x^{*}+x_{n}^{*}\right\|^{q}+\lim _{n \rightarrow \infty}\left|\left\|x^{*}+y_{n}^{*} \mid\right\|^{q}\right. \\
& \quad \geq\left(\left\|x^{*}\right\|^{q}+C \limsup _{n \rightarrow \infty}\left\|x_{n}^{*}\right\|_{M}^{q}\right)+\left.\lim _{n \rightarrow \infty}\left|\| x^{*}+y_{n}^{*}\right|\right|^{q} \\
& \quad \geq\left\|x^{*}\right\|^{q}+C \limsup _{n \rightarrow \infty}\left\|x_{n}^{*}\right\|_{M}^{q}+\left|\left\|x ^ { * } \left|\left\|^{q}=\left|x^{*}\right|^{q}+C \limsup _{n \rightarrow \infty}\right\| x_{n}^{*} \|_{M}^{q} .\right.\right.\right.
\end{aligned}
$$

This proves that $M$ is $|\cdot|$-asymptotically $p$-flat (with the same constant $C$ ).

For the proof of the statement about $\mathrm{K}^{*}$ norms, use the same definition for $|\cdot|$ and change sequences for nets and subsequences for subnets only in the first part of the previous argument.

Proposition 121 Let $(X,\|\cdot\|)$ be a Banach space. If a set $M \subset X$ is $\|\cdot\|$ asymptotically $p$-flat for some $p \in(1, \infty]$, then $M$ is also $\|\cdot\|$-asymptotically $p^{\prime}$-flat for every $p^{\prime} \in(1, p]$.

Proof. Given $f \in X^{*}$ and a $w^{*}$-null sequence $\left(f_{n}\right)$ in $X^{*}$, we have

$$
\limsup _{n \rightarrow \infty}\left\|f+f_{n}\right\|^{q} \geq\|f\|^{q}+C \limsup _{n \rightarrow \infty}\left\|f_{n}\right\|_{M}^{q},
$$


for some constant $C>0$. Then, having in mind that, for $q^{\prime} \geq q$ we have $\|\cdot\|_{\ell_{q}} \geq\|\cdot\|_{\ell^{\prime}}$, it follows that

$$
\begin{gathered}
\limsup _{n \rightarrow \infty}\left\|f+f_{n}\right\| \geq\left(\|f\|^{q}+C \limsup _{n \rightarrow \infty}\left\|f_{n}\right\|_{M}^{q}\right)^{1 / q} \\
\geq\left(\|f\|^{q^{\prime}}+C^{q^{\prime} / q} \limsup _{n \rightarrow \infty}\left\|f_{n}\right\|_{M}^{q^{\prime}}\right)^{1 / q^{\prime}},
\end{gathered}
$$

and hence

$$
\limsup _{n \rightarrow \infty}\left\|f+f_{n}\right\|^{q^{\prime}} \geq\|f\|^{q^{\prime}}+C^{q^{\prime} / q} \limsup _{n \rightarrow \infty}\left\|f_{n}\right\|_{M}^{q^{\prime}} .
$$

This proves that the set $M$ is $\|\cdot\|$-asymptotically $p^{\prime}$-flat.

The following proposition shows the quantitative connection between the concepts of being asymptotically $p$-flat and the $\mathrm{KK}^{*}$ property. This was, qualitatively explored in Proposition 117.

Proposition 122 Let $(X,\|\cdot\|)$ be a Banach space. Then, a bounded set $M \subset X$ is $\|\cdot\|$-asymptotically $p$-flat for some $p \in(1, \infty]$ if and only if the following holds: there exists $C>0$ such that for every $\varepsilon \in\left(0, C^{-q}\right)$ and for every sequence $\left(g_{n}\right)$ in $S_{X^{*}}$ such that $g_{n} \stackrel{w^{*}}{\longrightarrow} f \in X^{*}$ and $\left\|f-g_{n}\right\|_{M} \geq \varepsilon$, then $\|f\|^{q} \leq 1-C \varepsilon^{q}$, where $q=\frac{p}{p-1}$.

Proof. Assume first that $M$ is $\|\cdot\|$-asymptotically $p$-flat with a constant $C>0$. Take $\varepsilon \in\left(0, C^{-q}\right)$ and a sequence $\left(g_{n}\right)$ as in the statement. Put $f_{n}:=g_{n}-f$, $n \in \mathbb{N}$. Then $f_{n} \stackrel{w^{*}}{\longrightarrow} 0$, and $\left\|f_{n}\right\| \geq \varepsilon$ for all $n \in \mathbb{N}$. It follows from the definition of asymptotically $p$-flatness that $\lim \sup _{n \rightarrow \infty}\left\|f+f_{n}\right\|^{q} \geq\|f\|^{q}+C \lim \sup _{n \rightarrow \infty}\left\|f_{n}\right\|_{M}^{q}$. Then $1 \geq\|f\|^{q}+C \varepsilon^{q}$, so $\|f\|^{q} \leq 1-C \varepsilon^{q}$.

To prove the reverse implication, we shall argue by contradiction. Assume that $M$ is not $\|\cdot\|$-asymptotically $p$-flat. Then, for all $C>0$ there exists $f \in X^{*}$ and a $w^{*}$-null sequence $\left(f_{n}\right)$ in $X^{*}$ such that

$$
\limsup _{n \rightarrow \infty}\left\|f+f_{n}\right\|^{q}<\|f\|^{q}+C \limsup _{n \rightarrow \infty}\left\|f_{n}\right\|_{M}^{q} .
$$

Fix $C>0$ and find $f$ and $\left(f_{n}\right)$ accordingly.

We claim first that $\lim _{\sup } \rightarrow \infty\left\|f+f_{n}\right\| \neq 0$. If not, $\left(f+f_{n}\right) \stackrel{\|\cdot\|}{\longrightarrow} 0$. Since $\left(f+f_{n}\right) \stackrel{w^{*}}{\longrightarrow}$ $f$, we get $f=0$, so $f_{n} \stackrel{\|\cdot\|}{\longrightarrow} 0$. It follows that $0<0+C .0$, a contradiction. So $l:=\limsup _{n \rightarrow \infty}\left\|f+f_{n}\right\| \neq 0$. By choosing a subsequence of $\left(f_{n}\right)$, still denoted $\left(f_{n}\right)$, we may assume that $l=\lim _{n \rightarrow \infty}\left\|f+f_{n}\right\|$ and that $\left\|f+f_{n}\right\| \neq 0$ for all $n \in \mathbb{N}$. Put

$$
g_{n}:=\frac{f+f_{n}}{\left\|f+f_{n}\right\|}, \quad n \in \mathbb{N},
$$

and notice that $\left\|g_{n}\right\|=1$ and $g_{n} \stackrel{w^{*}}{\longrightarrow} f / l$.

We claim now that $\limsup _{n \rightarrow \infty}\left\|f_{n}\right\|_{M} \neq 0$. Indeed, if $\limsup _{n \rightarrow \infty}\left\|f_{n}\right\|_{M}=0$, we get, from (5.6), $\lim _{n}\left\|f+f_{n}\right\|^{q}<\|f\|^{q}$, and this is impossible since the dual norm is 
$w^{*}$-lower semicontinuous and $\left(f+f_{n}\right) \stackrel{w^{*}}{\longrightarrow} f$. Then the claim is proved. Without loss of generality, passing again to a subsequence, we may assume that

$$
\left\|f_{m}\right\|_{M}^{q}>\frac{1}{2} \limsup _{n \rightarrow \infty}\left\|f_{n}\right\|_{M}^{q} \quad \text { for all } \quad m \in \mathbb{N} .
$$

From (5.6) we get

$$
l^{q}<\|f\|^{q}+C \limsup _{n \rightarrow \infty}\left\|f_{n}\right\|_{M}^{q}
$$

So

$$
1<\left\|\frac{f}{l}\right\|^{q}+C \frac{\limsup _{n \rightarrow \infty}\left\|f_{n}\right\|_{M}^{q}}{l^{q}} .
$$

If we denote $\varepsilon:=\limsup _{n \rightarrow \infty}\left\|f_{n}\right\|_{M} /(2 l)$, the sequence $\left(g_{n}\right)$ defined in (5.7) satisfies

(i) $\left\|g_{n}\right\|=1$ for all $n \in \mathbb{N}$,

(ii) $g_{n} \stackrel{w^{*}}{\longrightarrow} f / l$,

(iii)

$$
\begin{aligned}
& \left\|\frac{f}{l}-g_{n}\right\|=\left\|\frac{f}{l}-\frac{f}{\left\|f+f_{n}\right\|}-\frac{f_{n}}{\left\|f+f_{n}\right\|}\right\|_{M} \\
& \quad \geq \frac{\left\|f_{n}\right\|_{M}}{\left\|f+f_{n}\right\|}-\|f\|_{M} \cdot\left|\frac{\left\|f+f_{n}\right\|-l}{l\left\|f+f_{n}\right\|}\right|>\frac{\lim \sup _{n \rightarrow \infty}\left\|f_{n}\right\|_{M}}{3 l}=\varepsilon
\end{aligned}
$$

for all $n \in \mathbb{N}$ big enough, whilst

(iv) $\|f / l\|^{q}>1-\varepsilon^{q} C^{\prime}$, where $C^{\prime}:=3^{q} C$.

This contradicts the condition in the statement.

The concept of modulus of convexity was introduced by Clarkson [Clark36]. The closely related modulus of smoothness was introduced by Day [Day41]. Precisely, they are defined in the following way.

Definition 123 Let $(X,\|\cdot\|)$ be a Banach space. We define the modulus of convexity (or rotundity) of $\|\cdot\|$ by

$$
\delta_{\|\cdot\|}(\varepsilon):=\inf \left\{1-\left\|\frac{x+y}{2}\right\| ; x, y \in B_{X},\|x-y\| \geq \varepsilon\right\}, \quad \varepsilon \in(0,2] .
$$

We say that the modulus of convexity has power type $q$ if there exists a constant $K>0$ such that $\delta_{\|\cdot\|}(\varepsilon) \geq K \varepsilon^{q}$ for all $\varepsilon \in(0,2]$.

Definition 124 Let $(X,\|\cdot\|)$ be a Banach space. We define the modulus of smoothness of $\|\cdot\|$ by

$$
\rho_{\|\cdot\|}(\tau):=\sup \left\{\frac{(\|x+\tau y\|-1)+(\|x-\tau y\|-1)}{2} ; x, y \in S_{X}\right\}, \quad \tau>0 .
$$

Let $p \geq 1$. We say that the modulus of smoothness $\rho_{\|\cdot\|}$ has power type $p$ if there exists a constant $K>0$ such that $\rho_{\|\cdot\|}(\tau) \leq K \tau^{p}$ for all $\tau>0$. 
Remark 125 Observe that, from Nördlander theorem [Di75, Theorem 3.3.1], it follows that there exists a positive constant $C>0$ such that for any convex space $X$, $\delta_{X}(\varepsilon) \leq C \varepsilon^{2}$ (see also [LT79, Proposition 1.e.5]). Lindenstrauss' duality formula (see, e.g., [LT79, Proposition 1.e.2.]) gives that $p \leq 2$ in the case of the modulus of smoothness.

The following result can be found in [FHHMPZ01, Lemma 9.2] and is due to Figiel.

Lemma $126\left([\right.$ Fig76] $)$ Let $(X,\|\cdot\|)$ be a Banach space and let $\delta_{\|\cdot\|}$ be the modulus of convexity of $\|\cdot\|$. Then $\delta_{\|\cdot\|}(\varepsilon)=\inf \left\{1-\left\|\frac{x+y}{2}\right\| ; x, y \in S_{X},\|x-y\|=\varepsilon\right\}$.

Remark 127 An obvious consequence of Lemma 126 is the following: denote by $\delta\left(S_{X}, \geq\right)$ the modulus of convexity defined by using $x, y \in S_{X}$ and $\|x-y\| \geq \varepsilon$ (this is formula (5.8)). Analogously, define $\delta\left(S_{X},=\right.$ ) or any other combination of $S_{X}, B_{X}$ for the first variable and $\geq$, = for the second variable. Lemma 126 says that $\delta\left(B_{X}, \geq\right)=\delta\left(S_{X},=\right)$. Then we have

$$
\begin{aligned}
& \delta\left(B_{X}, \geq\right) \leq \delta\left(B_{X},=\right) \leq \delta\left(S_{X},=\right) \\
& \delta\left(B_{X}, \geq\right) \leq \delta\left(S_{X}, \geq\right) \leq \delta\left(S_{X},=\right) .
\end{aligned}
$$

Hence, all modulus defined above coincide.

The following result (Proposition 129) provides a test for a Banach space having a closed unit ball $\|\cdot\|$-asymptotically $p$-flat for some $p \in(1,+\infty)$. In particular, it will follow that some of the classical spaces behave this way. We shall provide two alternative proofs. The second one relies on Proposition 122. It turns out that Proposition 129 is less general than the aforesaid proposition. However, the behavior of the modulus of convexity or smoothness of many spaces is well known, so the criterium appears to be useful.

Remark 128 At this point we think that it is important to mention the following. A previous approach to the theory developed here aiming at characterizing Banach spaces having injections from $\ell_{p}\left(\omega_{1}\right)$ and $c_{0}\left(\omega_{1}\right)$ into them has been done in [FGHZ03] and [FGMZ04] by using the modulus of smoothness. However, this approach does not and can not provide our general results in Theorems 156 and 158, since, as it was mentioned in Remark 128, there is no Banach space with a modulus of convexity of power type greater than 2 .

Proposition 129 Let $p \in(1,+\infty)$ and let $q:=\frac{p}{p-1}$ be the conjugate index. If the modulus of smoothness of $(X,\|\cdot\|)$ is of power type $p$, then $B_{X}$ is $\|\cdot\|$-asymptotically p-flat.

Proof. Let $p \in(1,+\infty)$. Assume that the modulus of smoothness of $(X,\|\cdot\|)$ is of power type $p$. Then, the modulus of convexity of $\left(X^{*},\|\cdot\|\right)$ is of power type $q$. This is a consequence of the basic relationship between both moduli due to Lindenstrauss (see, 
e.g., [FHHMPZ01, Lemma 9.9]). Since $q \in(1, \infty)$, note that, whenever $x^{*}, y^{*} \in B_{X^{*}}$, we have $\left\|\frac{1}{2}\left(x^{*}+y^{*}\right)\right\| \leq 1$, so $\left\|\frac{1}{2}\left(x^{*}+y^{*}\right)\right\|^{q} \leq\left\|\frac{1}{2}\left(x^{*}+y^{*}\right)\right\|$. Assume now that $\left\|x^{*}-y^{*}\right\| \geq \varepsilon \in(0,2]$. Then, due to the coincidence of the formulas used for the computation of the modulus of convexity (see Remark 127),

$$
1-\left\|\frac{1}{2}\left(x^{*}+y^{*}\right)\right\|^{q} \geq 1-\left\|\frac{1}{2}\left(x^{*}+y^{*}\right)\right\| \geq \delta_{\|\cdot\|}(\varepsilon) \geq c \varepsilon^{q} .
$$

Take $f \in B_{X^{*}}$ and $\left(g_{n}\right) \in S_{X^{*}}$, such that $g_{n} \stackrel{w^{*}}{\rightarrow} f$ and $\left\|f-g_{n}\right\| \geq \epsilon$ for every $n \in \mathbb{N}$. Then it follows from (5.10) that

$$
\left\|\frac{1}{2}\left(f+g_{n}\right)\right\|^{q} \leq 1-c \varepsilon^{q}, n \in \mathbb{N} .
$$

Since $\frac{1}{2}\left(f+g_{n}\right) \stackrel{w^{*}}{\rightarrow} f$, it follows from the $w^{*}$-lower semicontinuity of the dual norm that

$$
\|f\|^{q} \leq 1-c \varepsilon^{q} .
$$

By applying Proposition 122 we can conclude that $B_{X}$ is $\|\cdot\|$-asymptotically p-flat.

Corollary 130 On every superreflexive Banach space $(X,\|\cdot\|)$ there exists an equivalent norm ||$|\cdot|||$ and some $q \leq 2$ such that $B_{(X,\|\cdot \mid\|)}$ is ||$|\cdot|||$-asymptotically q-flat.

Proof. The Banach space $X^{*}$ is also superreflexive. A result of Pisier ([Pis74], see, e.g., [FHHMPZ01, Chapter 9]) says that every superreflexive Banach space can be renormed so that its modulus of convexity has power type $q$ for some $q \geq 2$. Then, the corresponding modulus of smoothness of $X$ has power type $p$ for some $p \in(1,2]$. It is enough to apply now Proposition 129.

Remark 131 1. Lancien [La95] proved that if $K$ is a scattered compact space of finite height then the unit ball of $C(K)$ is an asymptotically $\infty$-flat set in $C(K)$, via an equivalent norm (for some facts about scattered compacta see, for example, [FHHMPZ01, Chapter 12]). Therefore, for instance, the space $J L_{0}$ of Johnson and Lindenstrauss is an example of a space the unit ball of which is asymptotically $\infty$-flat, though it does not contain any isometric copy of $c_{0}\left(\omega_{1}\right)$. This space is not weakly Lindelöf determined (see, e.g., [FHHMPZ01, Theorem 12.58]). This should be compared with the statement of Theorem 156. Spaces considered there are always WCG.

2. Godefroy, Kalton, and Lancien [GKL00, Theorem 4.4] proved that the unit ball of a $W C G$ space $X$ of density character $\leq \omega_{1}$ is an asymptotically $\infty$-flat set if and only if $X$ is isomorphic to a subspace of $c_{0}(\Gamma)$ where $\# \Gamma=\omega_{1}$. 


\subsubsection{Some stability properties}

Obviously, if $X$ is a Banach space, $p \in(1,+\infty]$ and $M \subset X$ is an asymptotically $p$-flat set, then every subset of $M$ and the absolutely convex and closed hull of $M$ are also asymptotically $p$-flat sets. This was mentioned formally in Proposition 106.

Another stability result is given by the next result.

Proposition 132 Let $(X,\|\cdot\|)$ be a Banach space. Let $p \in(1, \infty]$. Let $M_{1}, M_{2}$ be asymptotically $p$-flat sets. Then $M_{1} \cup M_{2}$ and $M_{1}+M_{2}$ are asymptotically $p$-flat sets.

Proof. Let $f \in X^{*}$, and let $\left(f_{n}\right)$ be a $w^{*}$-null sequence in $X^{*}$. Find $C_{i}>0$ such that

$$
\limsup _{n \rightarrow \infty}\left\|f+f_{n}\right\|^{q} \geq\|f\|^{q}+C_{i} \limsup _{n \rightarrow \infty}\left\|f_{n}\right\|_{M_{i}}^{q}, \quad i=1,2 .
$$

Obviously, we may assume that $C_{1}=C_{2}(=: C)$ (just take $\left.C:=\min \left\{C_{1}, C_{2}\right\}\right)$. It is plain that $\left\|f_{n}\right\|_{M_{1} \cup M_{2}}^{q}=\max \left\{\left\|f_{n}\right\|_{M_{1}}^{q},\left\|f_{n}\right\|_{M_{2}}^{q}\right\}$.

We claim that, given real sequences $\left(s_{n}^{i}\right)_{n}, i=1,2$, we have

$$
\limsup _{n \rightarrow \infty} \max \left\{s_{n}^{1}, s_{n}^{2}\right\}=\max \left\{\limsup _{n \rightarrow \infty} s_{n}^{1}, \limsup _{n \rightarrow \infty} s_{n}^{2}\right\} .
$$

Indeed, if one of $l_{1}:=\lim \sup _{n \rightarrow \infty} s_{n}^{1}, l_{2}:=\lim \sup _{n \rightarrow \infty} s_{n}^{2}$ is $+\infty$, then (5.11) obviously holds. Assume now that neither $l_{1}$ nor $l_{2}$ is $+\infty$. Without loss of generality we may assume that $\max \left\{l_{1}, l_{2}\right\}=l_{2}$. Fix any $\varepsilon>0$. Then, there exists $n_{0} \in \mathbb{N}$ such that, for $n \geq n_{0}, s_{n}^{i}<l_{2}+\varepsilon, i=1,2$, and hence $\max \left\{s_{n}^{1}, s_{n}^{2}\right\}<l_{2}+\varepsilon$. Thus we get $\limsup _{n \rightarrow \infty} \max \left\{s_{n}^{1}, s_{n}^{2}\right\} \leq l_{2}+\varepsilon$. Since $\varepsilon>0$ was arbitrary, we obtain $\limsup _{n \rightarrow \infty} \max \left\{s_{n}^{1}, s_{n}^{2}\right\} \leq l_{2}$. The inequality " $\geq$ " in (5.11) is obvious. This proves the claim.

To finish the proof, observe that, according to (5.11),

$$
\begin{aligned}
& C \limsup _{n \rightarrow \infty}\left\|f_{n}\right\|_{M_{1} \cup M_{2}}^{q} \\
& \quad=C \max \left\{\limsup _{n \rightarrow \infty}\left\|f_{n}\right\|_{M_{1}}^{q}, \limsup _{n \rightarrow \infty}\left\|f_{n}\right\|_{M_{2}}^{q}\right\} \leq \limsup _{n \rightarrow \infty}\left\|f+f_{n}\right\|^{q}-\|f\|^{q},
\end{aligned}
$$

and this concludes the proof of the first part.

To prove the statement concerning sums, assume again that constants $C_{1}$ and $C_{2}$ in the definition of asymptotically $p$-flatness for $M_{1}$ and $M_{2}$ coincide. Notice that, for $g \in X^{*},\left|g\left(m_{1}+m_{2}\right)\right| \leq\left|g\left(m_{1}\right)\right|+\left|g\left(m_{2}\right)\right| \leq\|g\|_{M_{1}}+\|g\|_{M_{2}}$, for all $m_{i} \in M_{i}, i=1,2$, hence $\|g\|_{M_{1}+M_{2}} \leq\|g\|_{M_{1}}+\|g\|_{M_{2}}$. By Hölder inequality, we get

$$
\|g\|_{M_{1}}+\|g\|_{M_{2}} \leq 2^{1 / p}\left(\|g\|_{M_{1}}^{q}+\|g\|_{M_{2}}^{q}\right)^{1 / q},
$$

so

$$
\|g\|_{M_{1}+M_{2}}^{q} \leq 2^{q / p}\left(\|g\|_{M_{1}}^{q}+\|g\|_{M_{2}}^{q}\right)
$$


This holds for each $g:=f_{n}$, where $\left(f_{n}\right)$ is a $w^{*}$-null sequence in $X^{*}$. Then

$$
\begin{aligned}
& C \limsup _{n \rightarrow \infty}\left\|f_{n}\right\|_{M_{1}+M_{2}}^{q} \leq 2^{q / p}\left(C \limsup _{n \rightarrow \infty}\left\|f_{n}\right\|_{M_{1}}^{q}+C \underset{n \rightarrow \infty}{\limsup }\left\|f_{n}\right\|_{M_{2}}^{q}\right) \\
& \quad \leq 2^{q / p} 2\left(\limsup _{n \rightarrow \infty}\left\|f+f_{n}\right\|^{q}-\|f\|^{q}\right)=2^{q}\left(\limsup _{n \rightarrow \infty}\left\|f+f_{n}\right\|^{q}-\|f\|^{q}\right) .
\end{aligned}
$$

This shows that

$$
\limsup _{n}\left\|f+f_{n}\right\|^{q} \geq\|f\|^{q}+C 2^{-q} \limsup _{n \rightarrow \infty}\left\|f_{n}\right\|_{M_{1}+M_{2}}^{q},
$$

and this concludes the proof.

Proposition 133 Let $X$ and $Y$ be Banach spaces. Let $T: Y \rightarrow X$ be a continuous linear mapping. Let $M \subset Y$ be an asymptotically $p$-flat set, for some $p \in(1,+\infty]$. Then TM is asymptotically p-flat.

Proof. Let $\left\|x^{*}\right\|^{q}:=\left\|x^{*}\right\|^{q}+\left\|T^{*} x^{*}\right\|^{q}$ for every $x^{*} \in X^{*}$, where $q:=\frac{p}{p-1}$. A standard convexity argument and the fact that the function so defined on $X^{*}$ is $w^{*}$ lower semicontinuous proves that $\left|\|\cdot \mid\|\right.$ is an equivalent dual norm in $X^{*}$. We shall prove that $T M$ is $\left|\|\cdot \mid\|\right.$-asymptotically $p$-flat. Indeed, given $x^{*} \in X^{*}$ and a $w^{*}$-null sequence $\left(x_{n}^{*}\right)$ in $X^{*}$, we have, due to the $w^{*}$-lower semicontinuity of the dual norm,

$$
\begin{aligned}
& \limsup _{n \rightarrow \infty}\left\|x^{*}+x_{n}^{*}\right\|^{q} \\
& \quad=\limsup _{n \rightarrow \infty}\left(\left\|x^{*}+x_{n}^{*}\right\|^{q}+\left\|T^{*} x^{*}+T^{*} x_{n}^{*}\right\|^{q}\right) \\
& \quad \geq\left\|x^{*}\right\|^{q}+\limsup _{n \rightarrow \infty}\left\|T^{*} x^{*}+T^{*} x_{n}^{*}\right\|^{q} \\
& \quad \geq\left\|x^{*}\right\|^{q}+\left\|T^{*} x^{*}\right\|^{q}+C \limsup _{n \rightarrow \infty}\left\|T^{*} x_{n}^{*}\right\|_{M}^{q} \\
& \quad=\left\|x^{*}\right\|^{q}+C \limsup _{n \rightarrow \infty}\left\|x_{n}^{*}\right\|_{T M}^{q},
\end{aligned}
$$

since $M$ is $\|\cdot\|_{p}$-asymptotically $p$-flat (with constant $C$ ). It follows that $T M$ is $|\| \cdot|||$-asymptotically $p$-flat (with the same constant $C$ ).

Remark 134 The statement of the previous proposition (and its proof) needs to pass to an equivalent norm. This is unavoidable, as it can be seen by looking at Example 113. Indeed, the identity mapping $I:\left(c_{0},\|\cdot\|_{\infty}\right) \rightarrow\left(c_{0},\|\cdot\|_{\mathcal{D}}\right)$, where $\|\cdot\|_{\mathcal{D}}$ is Day's norm on $c_{0}$, is linear and continuous. The set $B_{\left(c_{0},\|\cdot\|_{\infty}\right)}$ is, according to Proposition $135,\|\cdot\|_{\infty}$-asymptotically $\infty$-flat. However, the set $I\left[B_{\left(c_{0},\|\cdot\|_{\infty}\right)}\right]$ is not $\|\cdot\|_{\mathcal{D}}$-asymptotically $\infty$-flat, since it contains a positive multiple of $B_{\left(X,\|\cdot\|_{\mathcal{D}}\right)}$, and we mentioned in the aforesaid example that this last set is not $\|\cdot\|_{\mathcal{D}}$-asymptotically $\infty$-flat. 


\subsubsection{Canonical examples}

We shall provide examples of Banach spaces which contain subsets (typically linearly dense) that are asymptotically $p$-flat for some $p \in(1,+\infty]$. We call the first group of examples canonical since even the terminology is inspired in the behavior of the canonical norm on sequence or function spaces.

Proposition 135 For any infinite set $\Gamma$, the closed unit ball of the space $c_{0}(\Gamma)$ is a $\|\cdot\|_{\infty}$-asymptotically $\infty$-flat set, with constant $C=1$.

Proof. Consider $f \in \ell_{1}(\Gamma)$ and let $\left(f_{n}\right)$ be a $w^{*}$-null sequence in $\ell_{1}(\Gamma)\left(=c_{0}(\Gamma)^{*}\right)$. Since every element in $\ell_{1}(\Gamma)$ has countable support, it is enough to assume that $\Gamma:=\mathbb{N}$. We shall write $c_{0}$ and $\ell_{1}$ instead of $c_{0}(\mathbb{N})$ and $\ell_{1}(\mathbb{N})$, respectively.

Fix $\varepsilon>0$. We know that $\sum_{k=1}^{\infty}|f(k)|=\|f\|_{1}<+\infty$, hence there exists $k_{0} \in \mathbb{N}$ such that $\sum_{k=k_{0}+1}^{\infty}|f(k)|<\varepsilon$. The sequence $\left(f_{n}\right)$ is $w^{*}$-null, hence we can find $n_{0} \in \mathbb{N}$ such that, for all $n \geq n_{0}$ we have $\left|f_{n}(k)\right|<\varepsilon / k_{0}$, for $k=1,2, \ldots, k_{0}$. Then, for $n \geq n_{0}$,

$$
\begin{aligned}
\| f+ & f_{n} \|_{1}=\sum_{k=1}^{k_{0}}\left|f(k)+f_{n}(k)\right|+\sum_{k=k_{0}+1}^{\infty}\left|f(k)+f_{n}(k)\right| \\
& \geq \sum_{k=1}^{k_{0}}|f(k)|-\sum_{k=1}^{k_{0}}\left|f_{n}(k)\right|+\sum_{k=k_{0}+1}^{\infty}\left|f_{n}(k)\right|-\sum_{k=k_{0}+1}^{\infty}|f(k)| \\
& \geq\|f\|_{1}-\sum_{k=k_{0}+1}^{\infty}|f(k)|-\varepsilon+\left\|f_{n}\right\|_{1}-\sum_{k=1}^{k_{0}}\left|f_{n}(k)\right|-\varepsilon \\
& \geq\|f\|_{1}-\varepsilon-\varepsilon+\left\|f_{n}\right\|_{1}-\varepsilon-\varepsilon .
\end{aligned}
$$

From here, and since $\varepsilon>0$ was arbitrary, it follows that

$$
\limsup _{n \rightarrow \infty}\left\|f+f_{n}\right\|_{1} \geq\|f\|_{1}+\limsup _{n \rightarrow \infty}\left\|f_{n}\right\|_{1} .
$$

We propose a second proof (say, a "predual" proof) of Proposition 135, this time based on Milman's characterization of spaces with 1-LKK* norm given in the Introduction to this chapter. We follow the notation there.

A second proof of Proposition 135. Let $\tau=1$. Fix $x \in S_{c_{0}(\Gamma)}$ and $\varepsilon>0$. We can find a finite set $\Gamma_{\varepsilon, x} \subset \Gamma$ such that $|x(\gamma)|<\varepsilon$ for all $\gamma \in \Gamma \backslash \Gamma_{\varepsilon, x}$. Let $Y_{\varepsilon, x}:=\overline{\operatorname{span}}\left\{e_{\gamma} ; \gamma \in \Gamma \backslash \Gamma_{\varepsilon, x}\right\}$. This is a finite-codimensional subspace of $c_{0}(\Gamma)$. Given $y \in Y_{\varepsilon, x}$ such that $\|y\| \leq 1$, we have. obviously, $\|x+y\| \leq 1+\varepsilon$, so $\|x+y\|-1 \leq \varepsilon$. It follows that $\bar{\rho}(1, x) \leq \varepsilon$. This is true for every $\varepsilon>0$. Hence $\bar{\rho}(1, x)=0$. This holds for every $x \in S_{c_{0}(\gamma)}$, so $\bar{\rho}(1)=0$.

Proposition 136 For $p \in(1,+\infty)$ and for any infinite set $\Gamma$, the closed unit ball of the space $\ell_{p}(\Gamma)$ is a $\|\cdot\|_{p}$-asymptotically $p$-flat set, with constant $C=1$. 
Proof. The proof is an adjustment of the one provided for Proposition 135. We work out the details. Proceeding as in that case, it is enough to work in $\ell_{p}(\mathbb{N})$. In order to simplify the notation, given $k_{0} \in \mathbb{N}$ and $g \in \ell_{p}(\mathbb{N})$, we shall write $g \uparrow_{k_{0}}$ for the element in $\ell_{p}\left(\left\{1, \ldots, k_{0}\right\}\right)$ defined as the restriction of the function $g$ to the set $\left\{1, \ldots, k_{0}\right\}$, and $g\left\lceil_{C k_{0}}\right.$ the restriction of $g$ to the set $\mathbb{N} \backslash\left\{1, \ldots, k_{0}\right\}$. Then, we have, for $f \in \ell_{q}(\mathbb{N})$ and a $w^{*}$-null sequence $\left(f_{n}\right)$ in $\ell_{q}(\mathbb{N})$,

$$
\left\|f+f_{n}\right\|_{q}^{q}=\left\|\left(f+f_{n}\right) \uparrow_{k_{0}}\right\|_{q}^{q}+\|\left(f+f_{n}\right)\left\lceil_{C k_{0}} \|_{q}^{q}\right.
$$

Fix any $\varepsilon>0$. We find $k_{0} \in \mathbb{N}$ such that $\left\|f \uparrow_{C k_{0}}\right\|_{q}<\varepsilon$, and then $n_{0} \in \mathbb{N}$, dependent on $\varepsilon, k_{0}$, such that, for all $n \geq n_{0},\left\|f_{n} \uparrow_{k_{0}}\right\|_{q}<\varepsilon$. Having in mind that we are dealing with norms both on $\ell_{q}\left(\left\{1, \ldots, k_{0}\right\}\right)$ and on $\ell_{q}\left\{k_{0}+1, \ldots\right\}$, we get, for $n \geq n_{0}$,

$$
\begin{aligned}
& \left\|\left(f+f_{n}\right) \uparrow_{k_{0}}\right\|_{q} \geq\left\|f \uparrow_{k_{0}}\right\|_{q}-\left\|f_{n} \Upsilon_{k_{0}}\right\|_{q} \\
& \quad \geq\|f\|_{q}-\| f\left\lceil_{C k_{0}}\left\|_{q}-\right\| f_{n} \Upsilon_{k_{0}}\left\|_{q} \geq\right\| f \|_{q}-2 \varepsilon\right.
\end{aligned}
$$

and, analogously,

$$
\|\left(f+f_{n}\right)\left\lceil_ { C k _ { 0 } } \| _ { q } \geq \| f _ { n } \| _ { q } - \| f _ { n } \Upsilon _ { k _ { 0 } } \| _ { q } - \| f \left\lceil_{C k_{0}}\left\|_{q} \geq\right\| f_{n} \|_{q}-2 \varepsilon .\right.\right.
$$

From (5.13), (5.14), and (5.15) we get, for $n \geq n_{0}$,

$$
\left\|f+f_{n}\right\|_{q}^{q} \geq\left(\|f\|_{q}-2 \varepsilon\right)^{q}+\left(\left\|f_{n}\right\|_{q}-2 \varepsilon\right)^{q} .
$$

It is possible to take limsup at both sides of equation (5.16) to get

$$
\limsup _{n \rightarrow \infty}\left\|f+f_{n}\right\|_{q}^{q} \geq\left(\|f\|_{q}-2 \varepsilon\right)^{q}+\limsup _{n}\left(\left\|f_{n}\right\|_{q}-2 \varepsilon\right)^{q} .
$$

Finally, let $\varepsilon$ go to 0 in $(5.17)$ to obtain the desired inequality.

Remark 137 It is well known that the spaces $\ell_{p}$, for $p \in[2,+\infty)$, have modulus of smoothness of power type 2 (see, e.g., [Xu91]), while for $1<p \leq 2$, they have modulus of smoothness of power type $p$. In view of Proposition 129, we get that $B_{\ell_{p}}$ is $\|\cdot\|_{p}$-asymptotically 2 -flat for $p \in[2,+\infty)$, while it is $\|\cdot\|_{p}$-asymptotically $p$-flat for $1<p \leq 2$. The result in Proposition 136 is more precise.

We denote by $L_{p}, p \in[1,+\infty)$, the space $L_{p}(\Omega, \Sigma, \mu)$ for a measurable space $(\Omega, \Sigma, \mu)$, with positive $\mu$, endowed with its canonical norm $\|f\|_{p}:=\left(\int_{\Omega}|f(\omega)|^{p} d \mu(\omega)\right)^{\frac{1}{p}}, f \in$ $L_{p}$. In order to prove that the closed unit ball $B_{L_{p}}$ of the space $L_{p}$ is $\|\cdot\|_{p^{-}}$ asymptotically $p$-flat, we use the following classical result (see, e.g., [Xu91] and [DGZ, Chapter V, Corollary 1.2]).

Proposition 138 (i) If $p \in(1,2]$, then the canonical norm on $L_{p}$ has modulus of smoothness of power type $p$ and modulus of convexity of power type 2 . 
(ii) If $p \in(2, \infty)$, then the canonical norm on $L_{p}$ has modulus of smoothness of power type 2 and modulus of convexity of power type $p$.

Corollary 139 Let $\mu$ be a positive measure on a measurable space $(\Omega, \Sigma)$ and let $p \in(1,+\infty)$. Let $B_{L_{p}}$ denote the closed unit ball of the space $L_{p}(\Omega, \Sigma, \mu)$. If $p \in(1,2)$,

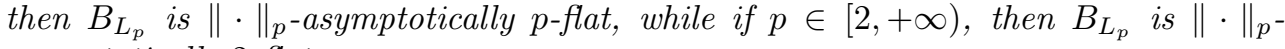
asymptotically 2 -flat.

Proof. Both statements are straightforward consequences of Propositions 129 and 138.

Remark 140 Note that Proposition 138 and Corollary 139 give only relevant information for the case $p \in[1,2)$. See also Remark 128. Of course, the natural conjecture is that for spaces $L_{p}$ a result similar to Proposition 136 holds. We were unable to prove or disprove this conjecture.

\subsubsection{Innerly asymptotically $p$-flat sets}

\subsubsection{Definitions}

We restrict now the definition of an asymptotically $p$-flat set to what we call an innerly asymptotically p-flat set, what allows us to get rid off the Asplundness condition demanded in Theorem 156. We will see below (Proposition 172) that an innerly asymptotically $p$-flat set has, somehow, a certain Asplund behavior itself, and it is this fact what allows to prove Theorem 158. To be precise, we introduce at this stage the following definition.

Definition 141 Let $X$ be a Banach space $X$, let $M \subset X$ be a nonempty set, let $p \in(1, \infty]$, and put $q:=\frac{p}{p-1}$. We say that $M$ is innerly asymptotically $p$-flat if it is bounded and there exists $C>0$ such that,

$$
\limsup _{n \rightarrow \infty}\left\|f+f_{n}\right\|_{M}^{q} \geq\|f\|_{M}^{q}+C \limsup _{n \rightarrow \infty}\left\|f_{n}\right\|_{M}^{q} .
$$

for every $f \in X^{*}$ and for every $w^{*}$-null sequence $\left(f_{n}\right)$ in $X^{*}$.

\subsubsection{Remarks and examples}

Remark 142 1. Notice that, in the previous definition, $C \in(0,1]$. Indeed, it is enough to take $f=0$ in Definition 141 . Hence $C \leq 1$. See also Remark 108 .

2. Certainly, being innerly asymptotically $p$-flat is invariant under equivalent renormings. In fact, the actual norm $\|\cdot\|$ on the Banach space $X$ does not play any role in the definition. Boundedness is also a concept that depends only on the dual pair $\left\langle X, X^{*}\right\rangle$. So, innerly asymptotically $p$-flatness is a concept that depends only on the dual pair. 
3. Let $(X,\|\cdot\|)$ be a Banach space. For the set $M:=B_{(X,\|\cdot\|)}$, the concepts of innerly asymptotically $p$-flatness and $\|\cdot\|$-asymptotically $p$-flatness obviously coincide.

Proposition 143 If a set $M$ is innerly asymptotically $p$-flat for some $p \in(1, \infty]$, then $M$ is also innerly asymptotically $p^{\prime}$-flat for every $p^{\prime} \in(1, p]$.

Proof. The proof is similar to the one provided for Proposition 121; so, we omit it.

The following proposition proves that norm-compact or, more generally, limited subsets of a Banach space, are innerly asymptotically $p$-flat for every $p \in(1, \infty]$. Of course, Propositions 114 and 115 follow as a consequence, in view of Proposition 145 below.

Proposition 144 Every norm-compact subset $M$ of a Banach space $(X,\|\cdot\|)$ is innerly asymptotically $p$-flat for every $p \in(1, \infty]$.

Proof. It is enough just to repeat, with small changes, the proof of Proposition 114.

Certainly, the concepts of innerly asymptotically $p$-flatness and asymptotic $p$-flatness are closely related. That one implies the other is shown precisely in the following result.

Proposition 145 Let $(X,\|\cdot\|)$ be a Banach space. Let $M \subset X$ be an innerly asymptotically $p$-flat set for some $p \in(1,+\infty]$ and let $C>0$ be the constant in (5.18). Then, there exists an equivalent norm $|\|\cdot \mid\|$ on $X$ such that $M$ is $|\|\cdot \mid\|$-asymptotically $p$-flat (with the same constant $C$ ) (i.e., $M$ is asymptotically p-flat).

Proof. Put $q=\frac{p}{p-1}$ and define

$$
\|f \mid\|^{q}:=\|f\|^{q}+\|f\|_{M}^{q}, \quad f \in X^{*} .
$$

This formula defines an equivalent dual norm. To check this, observe that ||$\cdot|| \mid$ is equivalent to the original norm because $M$ is a bounded set. It is a dual norm because the ||$|\cdot|||$-closed unit ball is $w^{*}$-closed, as it follows from the fact that $\left(\mathbb{R}^{2},\|\cdot\|_{\ell_{q}}\right)$ is a normed space, the $w^{*}$-lower semicontinuity of the dual norm $\|\cdot\|$ in $X^{*}$. The dual norm $\||\cdot|||$ on $X^{*}$ has the desired property. Indeed, given $f \in X^{*}$ and a $w^{*}$-null sequence $\left(f_{n}\right)$ in $X^{*}$, choose a subsequence $\left(g_{n}\right)$ of $\left(f_{n}\right)$ such that $\left\|g_{n}\right\|_{M} \rightarrow \lim \sup _{n \rightarrow \infty}\left\|f_{n}\right\|_{M}$, and that the sequences $\left(\left\|f+g_{n}\right\|\right)$ and $\left(\left\|f+g_{n}\right\|_{M}\right)$ both converge. It is possible to do so since the two last sequences are bounded. Then

$$
\begin{aligned}
& \limsup _{n \rightarrow \infty}\left\|f+f_{n} \mid\right\|^{q} \geq \lim _{n \rightarrow \infty}\left\|f+g_{n}\right\|^{q} \\
& \quad=\lim _{n \rightarrow \infty}\left\|f+g_{n}\right\|^{q}+\lim _{n \rightarrow \infty}\left\|f+g_{n}\right\|_{M}^{q} \\
& \quad \geq\|f\|^{q}+\|f\|_{M}^{q}+C \lim _{n}\left\|g_{n}\right\|_{M}^{q}=\|\| f\left\|^{q}+C \lim _{n \rightarrow \infty}\right\| g_{n} \|_{M}^{q} \\
& \quad=\|f \mid\|^{q}+C \limsup _{n \rightarrow \infty}\left\|f_{n}\right\|_{M}^{q} .
\end{aligned}
$$


Remark 146 From Proposition 145 it follows that the concept of being innerly asymptotically $p$-flat for some $p \in(1,+\infty]$ is stronger than being asymptotically $p$-flat. It is in fact strictly stronger, as follows from the example in Remark 161 below.

Remark 147 Since we provided an example (Example 113) of a Banach space $(X,|| \mid$. \|\|$)$ and a subset $M \subset X$ that is innerly asymptotically $p$-flat, and yet is not \|\|$\cdot \| \mid$ asymptotically $p$-flat, the renorming procedure in Proposition 145 is indeed unavoidable. There exists an equivalent norm $\|\cdot\|$ on $X$ such that the set $M$ is $\|\cdot\|-$ asymptotically $p$-flat, according to Proposition 145.

\subsubsection{Stability results}

The property of innerly asymptotically $p$-flatness, although crucial for the characterization of general Banach spaces that are $c_{0}\left(\omega_{1}\right)$ - $\left(\right.$ resp. $\ell_{p}\left(\omega_{1}\right)$-) generated (see Theorem 158), turns out to be quite unstable, in contrast with the behavior of the $\|\cdot\|$-asymptotically $p$-flatness. For example (and this is quite disturbing), let us consider the following simple remark, that we formulate as a proposition.

Proposition 148 A subset of an innerly asymptotically $p$-flat set does not need to be innerly asymptotically p-flat itself.

Proof. Fix $p \in(1,+\infty]$ and put $X:=c_{0}$ if $p=+\infty$ and $X:=\ell_{p}$ otherwise. We proved in Propositions 135 and 136, and in Remark 142.3 that the set $M:=B_{X}$ is innerly asymptotically $p$-flat as well as $\|\cdot\|$-asymptotically $p$-flat. Put $N=\left\{e_{1}, e_{2}, \ldots\right\}$, where the $e_{i}{ }^{\prime}$ s are the canonical unit vectors in $X$; thus $N \subset M$. Let $\left\{f_{1}, f_{2}, \ldots\right\}$ be the corresponding sequence of functional coefficients. We then have $\left\|f_{1}+f_{n}\right\|_{N}=1=$ $\left\|f_{1}\right\|_{N}=\left\|f_{n}\right\|_{N}$ for all $n=2,3, \ldots$ Thus (5.18) is violated no matter how small $C>0$ is. But notice that $N$ is still $\|\cdot\|$-asymptotically $p$-flat, according to Proposition 106.

Of course, the closed absolutely convex hull of an innerly asymptotically $p$-flat set is again an innerly asymptotically $p$-flat set. This follows from the very definition of the seminorm $\|\cdot\|_{M}$

Proposition 149 Let $X$ and $Y$ be Banach spaces. Let $T: Y \rightarrow X$ be a continuous linear mapping and let $M \subset Y$ be an innerly asymptotically $p$-flat set for some $p \in$ $(1,+\infty]$. Then $T M$ is again innerly asymptotically $p$-flat.

Proof. Observe first that, given $x^{*} \in X^{*}$, we have $\left\|x^{*}\right\|_{T M}=\left\|T^{*} x^{*}\right\|_{M}$. Second, if $\left(x_{n}^{*}\right)$ is a bounded sequence in $X^{*}$ that $w^{*}$-converges to 0 , then the sequence $\left(T^{*} x_{n}^{*}\right)$ is bounded and $w^{*}$-converges to 0 . It is enough now to refer to the very definition of innerly asymptotically $p$-flatness to conclude the proof. 


\subsection{Generating a space through an operator}

\subsubsection{Introduction}

Definition 150 We say that a Banach space $X$ is generated by a set $M \subset X$ if $M$ is linearly dense in $X$. The space $X$ is said to be generated by a Banach space $Y$ if there is a bounded linear operator from $Y$ into $X$ such that $T Y$ is dense in $X$.

Questions on generating spaces by, typically, Hilbert or superreflexive spaces were studied in [FGHZ03] and [FGMZ04] by using the moduli of uniform smoothness. Here we continue in this direction by using, in the Asplund setting, Kadec-Klee norms instead. This allows us to get a characterization also for $p>2$, where the former approach cannot work as there are no moduli of smoothness of power type better than 2. See Proposition 129 for a precise description of this, Remarks 127 and 128, and Corollary 130 for a consequence concerning superreflexivity.

\subsubsection{Remarks and examples}

Let us start by a simple observation.

Proposition 151 The property of a Banach space to be generated by another Banach space is transitive. i.e., assume that $X$ is a Banach space generated by a second Banach space $Y$, and that $Y$ in turn is generated by a third Banach space $Z$. Then $X$ is generated by $Z$.

Proof. Let $T: Y \rightarrow X$ and $S: Z \rightarrow Y$ be bounded linear operators with dense range. Then $T \circ S$ is a bounded linear operator from $Z$ into $X$ with dense range.

The following remark shows why we are interested mainly in non-separable Banach spaces.

Remark 152 It is a simple fact that every separable Banach space is generated by $c_{0}(\mathbb{N})$ and by $\ell_{p}(\mathbb{N})$ for all $p \in[1,+\infty]$. Even more, the generating operator $T$ can be chosen to be one-to-one. We provide details for the sake of completeness. Let $X$ be a separable Banach space. It has, by the classical Markushevich result ([M43], see, e.g., [FHHMPZ01, Theorem 6.41]), a countable Markushevich basis (in short, a countable M-basis) $\left\{x_{n} ; x_{n}^{*}\right\}_{n \in \mathbb{N}} \subset X \times X^{*}$. We may assume that $\left\|x_{n}\right\|=1$ for all $n \in \mathbb{N}$. We define $T: c_{0}(\mathbb{N}) \rightarrow X$ by the formula

$$
T c:=\sum_{n=1}^{\infty} a_{n} \frac{1}{2^{n}} x_{n}, \quad c:=\left(a_{n}\right) \in c_{0}(\mathbb{N}) .
$$

Obviously, $T$ is a linear operator. That it is well defined and continuous follows from the estimate

$$
\|T c\| \leq\|c\|_{\infty} \sum_{n=1}^{\infty} \frac{1}{2^{n}}=\|c\|_{\infty}, \quad c \in c_{0}(\mathbb{N}) .
$$


In order to see that $T$ is one-to-one, let $c \in c_{0}(\mathbb{N})$ be such that $T c=0$. Fix $m \in \mathbb{N}$. Then $\left\langle T c, x_{m}^{*}\right\rangle=a_{m}\left(1 / 2^{m}\right)=0$, hence $a_{m}=0$. This holds for all $m \in \mathbb{N}$. Since $\left\{x_{n} ; n \in \mathbb{N}\right\} \subset T c_{0}(\mathbb{N})$ the operator $T$ has dense range.

The previous proof works word by word for $\ell_{\infty}(\mathbb{N})$ instead of $c_{0}(\mathbb{N})$.

In the $\ell_{p}(\mathbb{N})$ case, for $p \in[1,+\infty)$, define an operator $T: \ell_{p}(\mathbb{N}) \rightarrow X$ by formally the same expression as before, i.e.,

$$
T l:=\sum_{n=1}^{\infty} l_{n} \frac{1}{2^{n}} x_{n}, \quad l:=\left(l_{n}\right) \in \ell_{p}(\mathbb{N}) .
$$

Again, $T$ is obviously linear. That it is well defined and continuous follows from the following estimate, where we use Hölder inequality.

$$
\begin{aligned}
\|T l\| & \leq \sum_{n=1}^{\infty}\left|l_{n}\right| \frac{1}{2^{n}} \\
& \leq\left(\sum_{n=1}^{\infty}\left|l_{n}\right|^{p}\right)^{1 / p}\left(\sum_{n=1}^{\infty}\left(\frac{1}{2^{q}}\right)^{n}\right)^{1 / q} \leq \frac{1}{2^{q}-1}\|l\|_{p}
\end{aligned}
$$

valid for every $l=\left(l_{n}\right) \in \ell_{p}(\mathbb{N})$. The same argument as above proves that $T$ is one-to-one and that it has dense range.

The following remark shows why we are not interested, even in the non-separable setting, in spaces which are $\ell_{1}(\Gamma)$-generated. The situation is completely different for the $p \in(1,+\infty]$-case, as it can be seen by looking at Theorems 156 and 158 .

Remark 153 Every Banach space is a quotient of a suitable $\ell_{1}(\Gamma)$. In order to see this, it is enough to extend the classical statement concerning separable Banach spaces and $\ell_{1}$ to (in general) a non-separable setting. In particular, every Banach space is $\ell_{1}(\Gamma)$-generated for some suitable set $\Gamma$. We can restrict a little bit the scope if we want a one-to-one mapping. Indeed, every Banach space $(X,\|\cdot\|)$ with an $M$-basis $\left\{x_{\gamma} ; f_{\gamma}\right\}_{\gamma \in \Gamma}$, is generated by the space $\ell_{1}(\Gamma)$. The proof of this fact follows the same pattern presented in Remark 152. Indeed, we may assume that $\left\|x_{\gamma}\right\|=1$ for all $\gamma \in \Gamma$. Let $T: \ell_{1}(\Gamma) \rightarrow X$ be defined by

$$
T l:=\sum_{\gamma \in \Gamma} l_{\gamma} x_{\gamma}, \quad l:=\left(l_{\gamma}\right) \in \ell_{1}(\Gamma) .
$$

Observe that the number of non-zero summands in the previous sum is less or equal that $\aleph_{0}$. The mapping $T$ is obviously linear, and it is well defined and continuous, since $\|T l\| \leq \sum_{\gamma \in \Gamma}\left|l_{\gamma}\right| \cdot\left\|x_{\gamma}\right\|=\sum_{\gamma \in \Gamma}\left|l_{\gamma}\right|=\|l\|_{1}$. It is, moreover, one-to-one, and, since $T \ell_{1}(\Gamma)$ contains $\left\{x_{\gamma} ; \gamma \in \Gamma\right\}$, has dense image.

In contrast with the former remark, let us recall the following well-known and useful fact (see, e.g., [HMVZ07, Theorem 5.3]. We provide here the proof for the sake of completeness. 
Theorem 154 Every Banach space $(X,\|\cdot\|)$ with an M-basis $\left\{x_{\gamma} ; f_{\gamma}\right\}_{\gamma \in \Gamma}$ linearly injects into $c_{0}(\Gamma)$.

Proof. Let $\left\{x_{\gamma} ; f_{\gamma}\right\}_{\gamma \in \Gamma}$ be an M-basis in $X \times X^{*}$ such that $\left\|f_{\gamma}\right\|=1$ for all $\gamma \in \Gamma$. The operator $T: X \rightarrow \ell_{\infty}(\Gamma)$ defined as $T(x)=\left(f_{\gamma}(x)\right)_{\gamma \in \Gamma}$ for all $x \in X$, is linear and bounded, and it has norm 1. Moreover, $T\left(\operatorname{span}\left\{x_{\gamma} ; \gamma \in \Gamma\right\}\right) \subset c_{0}(\Gamma) \subset \ell_{\infty}(\Gamma)$, and $c_{0}(\Gamma)$ is closed in $\ell_{\infty}(\Gamma)$.

Remark 155 Every separable Banach space has an M-basis, according to the classical Markushevich theorem, as we mentioned in Remark 152. The class of Banach spaces having M-bases is quite large. For example, it includes all WLD Banach spaces, as we actually proved in Theorem 50 .

\subsubsection{The results}

\subsubsection{The Asplund setting}

Theorem 156 Let $X$ be an Asplund space of density $\omega_{1}$ and let $p \in(1, \infty)$ be given. Then the following assertions are equivalent.

(i) $X$ is WCG and is generated by an asymptotically p-flat subset, resp. by an asymptotically $\infty$-flat subset.

(ii) $X$ is generated by $\ell_{p}\left(\omega_{1}\right)$, resp. by $c_{0}\left(\omega_{1}\right)$.

The last statement in the following corollary is in [JZ77].

Corollary 157 For $p \in(1,+\infty)$, any subspace of $\ell_{p}\left(\omega_{1}\right)$ is $\ell_{p}\left(\omega_{1}\right)$-generated. Every subspace of $c_{0}\left(\omega_{1}\right)$ is $c_{0}\left(\omega_{1}\right)$-generated. In particular, every subspace of $c_{0}\left(\omega_{1}\right)$ is $W C G$.

\subsubsection{The general setting}

Theorem 158 Let $X$ be a general Banach space of density $\omega_{1}$ and let $p \in(1, \infty)$ be given. Then the following assertions are equivalent.

(i) $X$ is WLD and is generated by an innerly asymptotically p-flat subset, resp. by an innerly asymptotically $\infty$-flat subset.

(ii) $X$ is generated by $\ell_{p}\left(\omega_{1}\right)$, resp. by $c_{0}\left(\omega_{1}\right)$.

Remark 159 That every subspace of $c_{0}\left(\omega_{1}\right)$ is WCG solved an open problem. It was not clear at the time whether every subspace of a WCG Banach space was again WCG. Rosenthal example [Ros74] provided a negative answer to this question (we included in Example 77 another instance of such a behavior). Today it is possible to obtain the last statement in Corollary 157 in a more direct way. First, every subspace of a WLD Banach space is again WLD (a consequence of the fact that the continuous image of a Corson compact topological space is itself Corson compact; we refer to our proof in Corollary 55, where this deep fact has been avoided). Secondly, $c_{0}\left(\omega_{1}\right)$ is 
Asplund (in fact, $c_{0}(\Gamma)$ is Asplund for every set $\Gamma$ ). Moreover, it is well known that the Asplund property is inherited to subspaces. It follows then that every subspace of $c_{0}(\Gamma)$ is both Asplund and WLD. This implies that it is WCG (see, e.g., [Fab97, Theorem 8.3.3]).

Remark 160 We mentioned in Remark 152 that every separable Banach space is generated by $c_{0}(\mathbb{N})$ and by $\ell_{p}(\mathbb{N})$ for $p \in[1,+\infty]$. In particular, $\ell_{1}(\mathbb{N})$ is generated by $c_{0}(\mathbb{N})$. According to Theorem 158 , there exists a bounded linearly dense set $M \subset \ell_{1}(\mathbb{N})$ that is innerly asymptotically $\infty$-flat. However, $B_{\ell_{1}}$ is not innerly asymptotically $\infty$-flat (what amounts to say that it is not asymptotically $\infty$-flat). Indeed, if $e_{n}, n \in \mathbb{N}$ are the canonical vectors in $c_{0}(\mathbb{N})\left(\subset \ell_{\infty}\right)$, then $\left\|e_{1}+e_{n}\right\|_{\infty}=1=\left\|e_{1}\right\|_{\infty}=$ $\left\|e_{n}\right\|_{\infty}$ for all $n=2,3 \ldots$, and hence the ball $B_{\ell_{1}}$ cannot be (innerly) asymptotically $\infty$-flat.

Remark 161 As a consequence of Theorems 156 and 158, we get that, if a WCG Asplund Banach space $X$ is generated by an asymptotically $p$-flat set $M$, then it is generated by an innerly asymptotically $p$-flat set $S$. However, those sets do not necessarily coincide. Indeed, the closed unit ball of $c_{0}\left(\omega_{1}\right)$ is, by Proposition $135,\|\cdot\|_{\infty^{-}}$ asymptotically $\infty$-flat (and then $\infty$-flat); it follows that the set $\mathrm{S}:=\left\{e_{\alpha} ; \alpha \in\left[\omega_{0}, \omega_{1}\right)\right\}$, where $e_{\alpha}$ denotes the $\alpha$-th canonical unit vector, is also $\|\cdot\|_{\infty}$-asymptotically $\infty$-flat (a property that is inherited by subsets). However, $S$ it is not innerly asymptotically $\infty$-flat, as it can be seen by fixing $\alpha \in\left[\omega_{0}, \omega_{1}\right)$ and taking in $\ell_{1}\left(\omega_{1}\right)$ the vector $f:=f_{\alpha}$ and the $w^{*}$-null sequence $\left(f_{n}:=f_{\alpha_{n}}\right)$, where $\left(\alpha_{n}\right)$ is an increasing sequence of ordinals in $\left[\omega_{0}, \omega_{1}\right)$ and $f_{\alpha}$ denotes the $\alpha$ 'th element of the set of functional coefficients. The same example was used in Proposition 148.

Remark 162 Concerning the first statement in Corollary 157, we note that it is not true that "every subspace of an $\ell_{p}\left(\omega_{1}\right)$-generated space is $\ell_{p}\left(\omega_{1}\right)$-generated". This is indicated by a Rosenthal's counterexample. He produced a non-WCG subspace $R$ of an $L_{1}(\mu)$ space with "big" probability measure $\mu$ [Ros74]. As it is well known, $L_{1}(\mu)$ is $L_{2}(\mu)$-generated, i.e. $\ell_{2}(\Gamma)$-generated (we provide in Appendix 6.2 a stronger statement). Yet $R$ is $\ell_{p}(\Gamma)$-generated for no $p \in(1,+\infty)$, since it is not WCG. However, it is worth noticing in this direction Corollary 169 below.

Remark 163 Given any $p \in(1,+\infty)$, then every $\ell_{p}(\Gamma)$-generated space $X$ is a subspace of a Hilbert generated space. Indeed, recall first that the canonical norm on every $\ell_{p}(\Gamma)$ space, for $1<p<+\infty$, is uniformly (Fréchet and hence) Gâteaux smooth. It follows then that $\left(B_{\ell_{q}(\Gamma)}, w\right)$ is a uniform Eberlein compact for $1<q<+\infty$ (see, e.g., [FGZ01]). Find a linear bounded operator $T: \ell_{p}(\Gamma) \rightarrow X$, with dense range. Then $T^{*}$ continuously injects $\left(B_{X^{*}}, w^{*}\right)$ into a multiple of (the uniform Eberlein compact space) $\left(B_{\ell_{q}(\Gamma)}, w\right)$, and hence $\left(B_{X^{*}}, w^{*}\right)$ itself is a uniform Eberlein compact space. Thus $C\left(\left(B_{X^{*}}, w^{*}\right)\right)$ is Hilbert generated ([FGHZ03]) and hence $X$ is a subspace of the Hilbert generated space $C\left(\left(B_{X^{*}}, w^{*}\right)\right)$. In particular, every subspace of an $\ell_{p}(\Gamma)$ generated space is a subspace of a Hilbert generated space, although we showed in Remark 162 that maybe it is not $\ell_{p}(\Gamma)$-generated itself. Note that for $2 \leq p<+\infty$ 
the space $\ell_{p}(\Gamma)$ is $\ell_{2}(\Gamma)$-generated (i.e., Hilbert-generated). Hence, for these $p$ 's we have a simple proof of the claim.

Remark 164 Spaces to which Theorem 156 applies are Asplund by the very statement. In the setting of Theorem 158, there is no such restriction. However, Proposition 172 says that the set $M$ from (i) in this theorem is an Asplund set, so, by [Fab97, Theorem 1.4.4], the space $X$ is Asplund generated. In the case that the set $M$ in question is the closed unit ball of the Banach space, the space is Asplund.

\subsubsection{Proofs}

In the proof of Theorem 156 we shall need [FMZ07, Proposition 15] (however, see Remarks 167 and 168). Since it is quite a recent result, we shall reproduce here the statement — without a proof (Proposition 166). We need first a definition, simply a quantification of the well-known concept of an Asplund set. Recall that a subset $A$ of a Banach space $X$ is called an Asplund set if it is bounded and the pseudometric space $\left(X^{*},\|\cdot\|_{N}\right)$ is separable for every countable set $N \subset A$ (see, e.g., [Fab97, Definition 1.4.1]).

Definition 165 Let $(X,\|\cdot\|)$ be a Banach space, and let $\varepsilon>0$. Given a nonempty set $A$ of $B_{X}$, we say that $B_{X^{*}}$ is $\|\cdot\|_{A^{-}-\varepsilon \text {-separable if there exists a countable set }}$ $C \subset B_{X^{*}}$ such that for every $x^{*} \in B_{X^{*}}$ there is $c \in C$ so that $\left\|x^{*}-c\right\|_{A}<\varepsilon$, that is, $B_{X^{*}}$ can be covered by open balls with centers in $C$ and with $\|\cdot\|_{A}$-radii $\varepsilon$. We say in

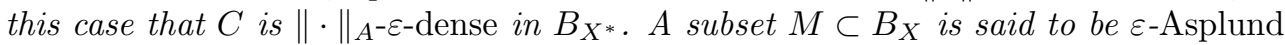
if for every countable subset $\emptyset \neq A \subset M$, the dual unit ball $B_{X^{*}}$ is $\|\cdot\|_{A}$-E-separable.

Clearly, if a set is $\varepsilon$-Asplund for every $\varepsilon>0$, then it is an Asplund set.

Proposition 166 (Proposition 15, [FMZ07]) Let $(Z,\|\cdot\|)$ be a non-separable Banach space admitting a linearly dense set $\Gamma \subset B_{Z}$ such that $\#\left\{\gamma \in \Gamma ;\left\langle\gamma, z^{*}\right\rangle \neq 0\right\}$ is at most countable for every $z^{*} \in Z^{*}$ (hence $Z$ is WLD). Assume that for every $n \in \mathbb{N}$ we have $\varepsilon_{n}>0$ and a closed convex symmetric $\varepsilon_{n}$-Asplund set $M_{n} \subset B_{Z}$. Then there exists a PRI $\left(P_{\alpha} ; \omega \leq \alpha \leq \mu\right)$ on $Z$ such that $P_{\alpha}\left(M_{n}\right) \subset M_{n}, P_{\alpha}(\gamma) \in\{\gamma, 0\}$ for every $\alpha \in[\omega, \mu]$, every $n \in \mathbb{N}$, and every $\gamma \in \Gamma$, and moreover, for every limit ordinal $\omega<\lambda \leq \mu$, every $n \in \mathbb{N}$, and every $z^{*} \in B_{Z^{*}}$ we have

$$
\limsup _{\beta \uparrow \lambda}\left\|P_{\lambda}^{*} z^{*}-P_{\beta}^{*} z^{*}\right\|_{M_{n}}<9 \varepsilon_{n} .
$$

\section{Proof of Theorem 156}

(i) $\Rightarrow$ (ii). Let $\|\cdot\|$ be an equivalent norm on $X$ and let $M \subset X$ be a linearly dense and $\|\cdot\|$-asymptotically $p$-flat set. Put $q=\frac{p}{p-1}$. A simple gymnastics with $M$ yields a new set - call it again $M$ - which is symmetric, convex, closed, still $\|\cdot\|$-asymptotically $p$-flat, and such that $M \subset B_{(X,\|\cdot\|)}$. Since $X$ is WCG, putting $M_{1}=M, M_{2}=M_{3}=$ $\cdots=B_{(X,\|\cdot\|)}$, and $\varepsilon=\frac{1}{n}, n \in \mathbb{N}$, in Proposition 166, we get a PRI $\left(P_{\alpha} ; \omega_{0} \leq \alpha \leq-\right.$ $\left.\omega_{1}\right)$ on $(X,\|\cdot\|)$ such that $\left(P_{\alpha}^{*} ; \omega_{0} \leq \alpha \leq \omega_{1}\right)$ is a PRI on the dual space $(X,\|\cdot\|)^{*}$, 
and moreover $P_{\alpha}(M) \subset M$ for every $\alpha \in\left(\omega_{0}, \omega_{1}\right)$; recall that $P_{\omega_{0}} \equiv 0$. We note that $\bigcup_{\omega_{0} \leq \alpha<\omega_{1}} P_{\alpha}^{*} X^{*}=X^{*}$. Indeed, given $f \in X^{*}$, we have $\left\|P_{\alpha}^{*} f-f\right\| \rightarrow 0$ as $\alpha \uparrow \omega_{1}$. We can find then an increasing sequence $\left(\alpha_{n}\right)$ in $\left[\omega_{0}, \omega_{1}\right)$ such that $\left\|P_{\alpha_{n}}^{*} f-f\right\| \rightarrow 0$ whenever $n \rightarrow \infty$. It follows that, if $\alpha:=\sup \left\{\alpha_{n} ; n \in \mathbb{N}\right\}\left(<\omega_{1}\right)$, then $f \in P_{\alpha}^{*} X^{*}$. Let $C>0$ witness that $M$ is $\|\cdot\|$-asymptotically $p$-flat, see Definition 1 . Nothing will happen if we take $C \in(0,1)$.

Claim 1. For every $0 \neq f \in X^{*}$, every $\varepsilon>0$, and every $\alpha \in\left[\omega_{0}, \omega_{1}\right)$, there is $\gamma_{f, \varepsilon, \alpha} \in\left(\alpha, \omega_{1}\right)$ such that

$$
\|f+g\|^{q} \geq(1-\varepsilon)\|f\|^{q}+C\|g\|_{M}^{q} \quad \text { whenever } \quad g \in \operatorname{ker} P_{\gamma_{f, \varepsilon, \alpha}^{*}}^{\text {and }} \quad\|g\|<\frac{1}{\varepsilon} .
$$

Proof. Fix any $0 \neq f \in X^{*}, \varepsilon>0$, and $\alpha \in\left[\omega_{0}, \omega_{1}\right)$. Assume that the claim does not hold for this triple. Find then $g_{1} \in \operatorname{ker} P_{\alpha+1}^{*}$ so that $\left\|g_{1}\right\|<\frac{1}{\varepsilon}$ and $\left\|f+g_{1}\right\|^{q}<$ $(1-\varepsilon)\|f\|^{q}+C\left\|g_{1}\right\|_{M}^{q}$. Further, find $\alpha_{1} \in\left(\alpha+1, \omega_{1}\right)$ such that $g_{1} \in P_{\alpha_{1}}^{*} X^{*}$. Find then $g_{2} \in \operatorname{ker} P_{\alpha_{1}}^{*}$ so that $\left\|g_{2}\right\|<\frac{1}{\varepsilon}$ and $\left\|f+g_{2}\right\|^{q}<(1-\varepsilon)\|f\|^{q}+C\left\|g_{2}\right\|_{M}^{q}$. Find $\alpha_{2} \in\left(\alpha_{1}, \omega_{1}\right)$ so that $g_{2} \in P_{\alpha_{2}}^{*} X^{*}$. ... Find $g_{n+1} \in \operatorname{ker} P_{\alpha_{n}}^{*}$ so that $\left\|g_{n+1}\right\|<\frac{1}{\varepsilon}$ and $\left\|f+g_{n+1}\right\|^{q}<(1-\varepsilon)\|f\|^{q}+C\left\|g_{n+1}\right\|_{M}^{q}$. Find then $\alpha_{n+1} \in\left(\alpha_{n}, \omega_{1}\right)$ so that $g_{n+1} \in P_{\alpha_{n+1}}^{*} X^{*}$. ... Thus we get an infinite sequence $g_{1}, g_{2}, \ldots$ in $X^{*}$ and an increasing sequence $\alpha_{1}<\alpha_{2}<\cdots<\omega_{1}$. The sequence $\left(g_{n}\right)$ is $w^{*}$-null. Indeed, put $\lambda=\lim _{n \rightarrow \infty} \alpha_{n}$; we still have $\lambda<\omega_{1}$. Fix any $x \in X$. Then for every $n \in \mathbb{N}$ we get

$$
\begin{aligned}
& \left|\left\langle x, g_{n+1}\right\rangle\right|=\left|\left\langle x, P_{\lambda}^{*}\left(g_{n+1}\right)\right\rangle\right|=\left|\left\langle P_{\lambda} x, g_{n+1}\right\rangle\right| \\
& =\left|\left\langle P_{\lambda} x-P_{\alpha_{n}} x, g_{n+1}\right\rangle\right| \leq\left\|P_{\lambda} x-P_{\alpha_{n}} x\right\| \cdot \frac{1}{\varepsilon} .
\end{aligned}
$$

Hence $\left\langle x, g_{n}\right\rangle \rightarrow 0$ as $n \rightarrow \infty$. Therefore, by (5.5), we have

$$
\begin{gathered}
\limsup _{n \rightarrow \infty}\left\|f+g_{n}\right\|^{q} \geq\|f\|^{q}+C \underset{n \rightarrow \infty}{\limsup }\left\|g_{n}\right\|_{M}^{q} \\
\left(>(1-\varepsilon)\|f\|^{q}+C \limsup _{n \rightarrow \infty}\left\|g_{n}\right\|_{M}^{q} \geq \limsup _{n \rightarrow \infty}\left\|f+g_{n}\right\|^{q}\right),
\end{gathered}
$$

a contradiction.

Claim 2. For every $\alpha \in\left[\omega_{0}, \omega_{1}\right)$ there exists $\beta_{\alpha} \in\left(\alpha, \omega_{1}\right)$ such that

$$
\|f+g\|^{q} \geq\|f\|^{q}+C\|g\|_{M}^{q} \quad \text { whenever } f \in P_{\alpha}^{*} X^{*} \quad \text { and } g \in \operatorname{Ker} P_{\beta_{\alpha}}^{*} .
$$

Proof. Fix any $\alpha \in\left[\omega_{0}, \omega_{1}\right)$. Let $S$ be a countable dense subset in the (separable) subspace $P_{\alpha}^{*} X^{*}$. Using Claim 1, put then $\beta_{\alpha}=\sup \left\{\gamma_{f, 1 / n, \alpha} ; f \in S, n \in \mathbb{N}\right\}$. It is easy to check that this ordinal works.

Claim 3. There exists an increasing long sequence $\left(\delta_{\alpha}\right)_{\omega_{0} \leq \alpha \leq \omega_{1}}$ in $\left[\omega_{0}, \omega_{1}\right]$, with $\delta_{\omega_{0}}:=\omega_{0}$ and $\delta_{\omega_{1}}:=\omega_{1}$, and such that for every $\alpha \in\left[\omega_{0}, \omega_{1}\right)$ we have

$$
\|f+g\|^{q} \geq\|f\|^{q}+C\|g\|_{M}^{q} \quad \text { whenever } f \in P_{\delta_{\alpha}}^{*} X^{*} \text { and } g \in \operatorname{ker} P_{\delta_{\alpha}}^{*} .
$$


Proof. We shall prove first that there exists an increasing long sequence (call it, for the moment being, $\left.\left(\delta_{\alpha}\right)_{\omega_{0} \leq \alpha \leq \omega_{1}}\right)$ such that

$$
\|f+g\|^{q} \geq\|f\|^{q}+C\|g\|_{M}^{q} \text { whenever } f \in P_{\delta_{\alpha}}^{*} X^{*} \text { and } g \in \operatorname{ker} P_{\delta_{\alpha+1}^{*}}^{*}
$$

and, moreover, if $\alpha$ is a limit ordinal then $\delta_{\alpha}$ is also a limit ordinal and (5.21) holds. To this end, fix any $\alpha \in\left(\omega_{0}, \omega_{1}\right)$, and assume that we have already constructed ordinals $\delta_{\beta}$ 's verifying (5.22) for all $\beta \in\left[\omega_{0}, \alpha\right)$. If $\alpha$ has a predecessor, say $\alpha-1$, then, using Claim 2, put $\delta_{\alpha}=\beta_{\delta_{\alpha-1}}$. This ensures (5.22) for $f \in P_{\delta_{\alpha-1}}^{*} X^{*}$ and $g \in \operatorname{ker} P_{\delta_{\alpha}}^{*}$. Assume now that $\alpha$ is a limit ordinal. In this case, put simply $\delta_{\alpha}=\lim _{\beta \uparrow \alpha} \delta_{\beta}$ (observe that, in this case, $\delta_{\alpha}$ is a limit ordinal). Given $f \in P_{\delta_{\alpha}}^{*} X^{*}$ and $g \in \operatorname{ker} P_{\delta_{\alpha}}^{*}$, we have $\left\|P_{\delta_{\beta}}^{*} f+g\right\|^{q} \geq\left\|P_{\delta_{\beta}}^{*} f\right\|^{q}+C\|g\|_{M}^{q}$ for every $\beta<\alpha$. The net $\left(P_{\delta_{\beta}}^{*} f\right)_{\beta<\alpha}$ is $\|\cdot\|$-convergent to $f$, since $\left(P_{\gamma}^{*}\right)_{\omega_{0} \leq \gamma \leq \omega_{1}}$ is a PRI on $\left(X^{*},\|\cdot\|\right)$. This proves $(5.21)$.

Select from the long sequence $\left(\delta_{\alpha}\right)_{\omega_{0} \leq \alpha \leq \omega_{1}}$ only limit ordinals indices $\alpha$ 's. Now, it is enough to recall that the order type of the set of all limit ordinals in $\left[\omega_{0}, \omega_{1}\right)$ coincides with $\omega_{1}$. The long sequence so obtained (that we call again $\left(\delta_{\alpha}\right)_{\omega_{0} \leq \alpha \leq \omega_{1}}$ ) satisfies (5.21).

Claim 4. There exists a linear, bounded, injective and $w^{*}-$ to- $w^{*}$-continuous operator from $X^{*}$ into $\ell_{q}\left(\mathbb{N} \times\left[\omega_{0}, \omega_{1}\right)\right)$.

Proof. For each $\alpha \in\left[\omega_{0}, \omega_{1}\right)$ find a countable dense set $\left\{v_{1}^{\alpha}, v_{2}^{\alpha}, \ldots\right\}$ in $\frac{1}{2}\left(P_{\delta_{\alpha+1}}-\right.$ $\left.P_{\delta_{\alpha}}\right)(M)(\subset M)$. Define $T: X^{*} \rightarrow \mathbb{R}^{\mathbb{N} \times\left[\omega_{0}, \omega_{1}\right)}$ by

$$
T f(i, \alpha)=2^{-i} f\left(v_{i}^{\alpha}\right), \quad(i, \alpha) \in \mathbb{N} \times\left[\omega_{0}, \omega_{1}\right), \quad f \in X^{*} .
$$

Clearly, $T$ is linear and $w^{*}$-to-pointwise continuous. $T$ is injective because $\left(P_{\delta_{\alpha}} ; \alpha \in\right.$ $\left.\left[\omega_{0}, \omega_{1}\right]\right)$ is clearly a PRI on $X$. We shall show that the range of $T$ is a subset of the Banach space $\ell_{q}\left(\mathbb{N} \times\left[\omega_{0}, \omega_{1}\right)\right)$ and that $T$ is actually a bounded linear operator from $X^{*}$ to the latter space. Denote by $Y$ the linear span of the set $\bigcup_{\omega_{0} \leq \alpha<\omega_{1}}\left(P_{\delta_{\alpha+1}}^{*}-P_{\delta_{\alpha}}^{*}\right) X^{*}$. Take any $f \in Y$. Then we can write $f$ in the form $f=f_{1}+f_{2}+\cdots+f_{k}$ where $f_{j} \in\left(P_{\delta_{\alpha_{j}+1}}^{*}-P_{\delta_{\alpha_{j}}}^{*}\right) X^{*}, j=1, \ldots, k$, and $\alpha_{1}<\alpha_{2}<\cdots<\alpha_{k}$. Observing that $\delta_{\alpha_{1}}<\delta_{\alpha_{2}}<\cdots<\delta_{\alpha_{k}}$, we use (5.21) repeatedly, and thus we get

$$
\begin{aligned}
\frac{1}{C}\|f\|^{q} & =\frac{1}{C}\left\|\sum_{j=1}^{k} f_{j}\right\|^{q} \\
& \geq \frac{1}{C}\left\|\sum_{j=1}^{k-1} f_{j}\right\|^{q}+\left\|f_{k}\right\|_{M}^{q} \geq \frac{1}{C}\left\|\sum_{j=1}^{k-2} f_{j}\right\|^{q}+\left\|f_{k-1}\right\|_{M}^{q}+\left\|f_{k}\right\|_{M}^{q} \\
& \geq \ldots \geq \frac{1}{C}\left\|f_{1}\right\|^{q}+\sum_{j=2}^{k}\left\|f_{j}\right\|_{M}^{q} \geq \sum_{j=1}^{k}\left\|f_{j}\right\|_{M}^{q} \geq \sum_{i=1}^{\infty} 2^{-i q} \cdot \sum_{j=1}^{k}\left\|f_{j}\right\|_{M}^{q} \\
& \geq \sum_{i=1}^{\infty} 2^{-i q} \sum_{j=1}^{k}\left|f_{j}\left(v_{i}^{\alpha_{j}}\right)\right|^{q} \geq \sum_{i=1}^{\infty} \sum_{\alpha \in\left[0, \omega_{1}\right)} 2^{-i q}\left|f\left(v_{i}^{\alpha}\right)\right|^{q}=\|T f\|_{\ell_{q}}^{q}
\end{aligned}
$$


Therefore $T f \in \ell_{q}\left(\mathbb{N} \times\left[\omega_{0}, \omega_{1}\right)\right)$ for every $f \in Y$, and so $T(Y) \subset \ell_{q}\left(\mathbb{N} \times\left[\omega_{0}, \omega_{1}\right)\right)$. Now, it follows easily from the properties of $P_{\alpha}^{*}$ 's that $Y$ is norm-dense in $X^{*}$. Notice that $T 1_{Y}$ is a bounded linear operator from $Y$ into $\ell_{q}\left(\mathbb{N} \times\left[\omega_{0}, \omega_{1}\right)\right)$, so it has a bounded linear extension $\widetilde{T}$ to $X^{*}$, with values in $\ell_{q}\left(\mathbb{N} \times\left[\omega_{0}, \omega_{1}\right)\right)$, and with the same norm. Since $T: X^{*} \rightarrow \mathbb{R}^{\mathbb{N} \times\left[\omega_{0}, \omega_{1}\right)}$ is pointwise continuous, we easily get that $T=\widetilde{T}$. We thus proved that $T\left(X^{*}\right) \subset \ell_{q}\left(\mathbb{N} \times\left[\omega_{0}, \omega_{1}\right)\right)$.

Let $u$ be an element of $\ell_{p}\left(\mathbb{N} \times\left[\omega_{0}, \omega_{1}\right)\right)$ (resp. of $\left.c_{0}\left(\mathbb{N} \times\left[\omega_{0}, \omega_{1}\right)\right)\right)$. In order to prove the $w^{*}$-continuity of the functional $u \circ T: X^{*} \rightarrow \mathbb{R}$ defined by $u \circ T\left(x^{*}\right)=\left\langle u, T x^{*}\right\rangle, x^{*} \in$ $X^{*}$, it suffices, by the Banach-Dieudonné theorem, to check the $w^{*}$-continuity of $u \circ T$ restricted to $B_{X^{*}}$. But, on the (bounded) set $T B_{X^{*}}$, the topology of pointwise convergence coincides with the $w^{*}$-topology. Hence the $w^{*}$-to-pointwise continuity of $T$ gives that $u \circ T$ is $w^{*}$-continuous. It then follows that $T$ is $w^{*}$-to- $w^{*}$-continuous and Claim 4 is thus proved.

Finally, from the above, we can conclude that the adjoint operator $T^{*}$ goes from $\ell_{p}\left(\mathbb{N} \times\left[\omega_{0}, \omega_{1}\right)\right)\left(\right.$ resp., $c_{0}\left(\mathbb{N} \times\left[\omega_{0}, \omega_{1}\right)\right)$ into $X$. And since, $T$ is injective, $T^{*}\left(\ell_{p}(\mathbb{N} \times\right.$ $\left.\left[\omega_{0}, \omega_{1}\right)\right)$ (resp., $T^{*}\left(c_{0}\left(\mathbb{N} \times\left[\omega_{0}, \omega_{1}\right)\right)\right)$ ) is dense in $X$ and (ii) is proved.

(ii) $\Rightarrow($ i) This will follow immediately from the implication (ii) $\Rightarrow($ i) in Theorem 158 (the proof of this implication does not depend on Theorem 156). Indeed, assume that $X$ is a WCG Asplund space of density $\omega_{1}$ that is generated by $\ell_{p}\left(\omega_{1}\right)$ for some $p \in(1,+\infty]$. By Theorem $158, X$ is generated by an innerly asymptotically $p$-flat set $M$. According to Proposition $145, M$ is an asymptotically $p$-flat set (notice that, in general, in a different equivalent norm). And this is (i) in Theorem 156.

Remark 167 In the proof of Theorem 156, it is possible to avoid the use of Proposition 166 and to refer to results that are treated in this Memoir. Precisely, since $X$ is WCG and Asplund, there exists a shrinking M-basis $\left\{x_{i} ; x_{i}^{*}\right\}_{i \in I}$ in $X \times X^{*}$. The set $\left\{x_{i} ; i \in I\right\}$ countably supports $X^{*}$. Then (Proposition 30) there exists a PRI $\left(P_{\alpha}\right)_{\omega_{0} \leq \alpha \leq \mu}$ on $X$ subordinated to $\left\{x_{i} ; i \in I\right\}$ such that $P_{\alpha} M \subset M$ for every $\alpha \in\left[\omega_{0}, \mu\right]$. By Proposition 37, the PRI is shrinking, and this means that $\left(P_{\alpha}^{*}\right)_{\omega_{0} \leq \alpha \leq \mu}$ is a PRI on $X^{*}$.

Remark 168 Still another way to avoid the use of Proposition 166 is the following. Since $X$ is WCG and Asplund, it has an equivalent norm $|\|\cdot \mid\|$ the dual of which is LUR (see, e.g., [FHHMPZ01, Theorem 11.22]) and so $(X,\||\cdot|\|)$ is $\mathrm{K}^{*}$ (see, e.g., [FHHMPZ01, Exercise 8.83]).

By Proposition 120 we may assume that the norm $\|\cdot\|$ on $X$ that makes $M$ asymptotically $p$-flat is also $\mathrm{K}^{*}$. Let $\left(P_{\alpha}\right)_{\omega_{0} \leq \alpha \leq \mu}$ be a PRI on $X$ given by Proposition 30 . Given $f \in X^{*}$, and a limit ordinal $\beta \leq \omega_{1}$, then $P_{\alpha}^{*} f \stackrel{w^{*}}{\longrightarrow} P_{\beta}^{*} f$ whenever $\alpha \uparrow \beta$. At the same time, $\left\|P_{\alpha}^{*} f\right\| \leq\left\|P_{\beta}^{*} f\right\|$ for all $\alpha \leq \beta$, hence, by the $\mathrm{K}^{*}$ property of the norm, we get $P_{\alpha}^{*} f \stackrel{\|\cdot\|}{\longrightarrow} P_{\beta}^{*} f$, and so

$$
P_{\beta}^{*} X^{*}=\varlimsup_{\alpha<\beta} P_{\alpha}^{*} X^{*} \cdot \| \text {, for every limit ordinal } \beta \leq \omega_{1} .
$$


This is what was needed in order to make the former proof work.

Proof of Corollary 157. Fix $p \in(1,+\infty]$ and put $q=\frac{p}{p-1}$. Let $(X,\|\cdot\|)$ be a subspace of $\ell_{p}\left(\omega_{1}\right)$. Let $Q: \ell_{q}\left(\omega_{1}\right) \rightarrow X^{*}$ be the canonical quotient mapping. The

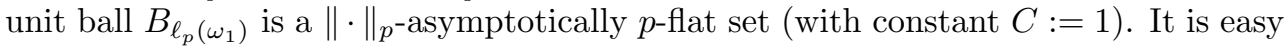
to prove that $B_{X}$ is also a $\|\cdot\|$-asymptotically $p$-flat set in $X$. Indeed, if $x^{*} \in X^{*}$ and $\left(x_{n}^{*}\right)$ is a $w^{*}$-null sequence in $X^{*}$, select first a subsequence $\left(x_{n_{k}}^{*}\right)$ of $\left(x_{n}^{*}\right)$ such that $\left\|x_{n_{k}}^{*}\right\| \rightarrow_{k} \lim \sup _{n \rightarrow \infty}\left\|x_{n}^{*}\right\|$. Let $\left(l_{k}^{*}\right)$ be a bounded sequence in $\ell_{q}\left(\omega_{1}\right)$ such that $Q l_{k}^{*}=x^{*}+x_{n_{k}}^{*}$ and $\left\|l_{k}^{*}\right\|=\left\|x^{*}+x_{n_{k}}^{*}\right\|$ for all $k \in \mathbb{N}$. The countability of the supports allows us to select a further subsequence $\left(l_{n_{k_{j}}}^{*}\right)$ of $\left(l_{n_{k}}^{*}\right)$ that is $w^{*}$-convergent to some $l^{*} \in \ell_{q}\left(\omega_{1}\right)$. Obviously, $Q l^{*}=x^{*}$. Then,

$$
\begin{aligned}
& \limsup _{n \rightarrow \infty}\left\|x^{*}+x_{n}^{*}\right\|^{q} \geq \limsup _{j \rightarrow \infty}\left\|x^{*}+x_{n_{k_{j}}}^{*}\right\|^{q} \\
& \quad=\limsup _{j \rightarrow \infty}\left\|l_{k_{j}}^{*}\right\|^{q}=\limsup _{j \rightarrow \infty}\left\|l^{*}+\left(l_{k_{j}}^{*}-l^{*}\right)\right\|^{q} \geq\left\|l^{*}\right\|^{q}+\limsup _{j \rightarrow \infty}\left\|l_{k_{j}}^{*}-l^{*}\right\|^{q} \\
& \quad \geq\left\|l^{*}\right\|^{q}+\lim _{n \rightarrow \infty}\left\|x_{n_{k_{j}}}^{*}\right\|^{q} \geq\left\|x^{*}\right\|+\limsup _{n \rightarrow \infty}\left\|x_{n}^{*}\right\|^{q} .
\end{aligned}
$$

We obtained that $B_{X}$ is $\|\cdot\|$-asymptotically $p$-flat. It is enough now to apply Theorem 156.

A look at the proof of Corollary 157 allows us to formulate a result a little bit more general. We have the following corollary, that should be compared with Remark 162 .

Corollary 169 Let $p \in(1,+\infty]$ and let $X$ be a subspace of a Banach space $Z$ such that $B_{Z}$ is asymptotically $p$-flat and $\left(B_{Z^{*}}, w^{*}\right)$ is angelic (more generally, $w^{*}$ sequentially compact). Then, $B_{X}$ is also asymptotically $p$-flat. In particular, if $Z$ is moreover $W L D$, then $X$ is $\ell_{p}\left(\omega_{1}\right)$-generated.

Proof. This time, and following the notation and the proof of Corollary 157, the angelicity of $\left(B_{Z^{*}}, w^{*}\right)$ allows us to select a $w^{*}$-convergent subsequence, instead of the argument involving the countability of the supports. The second part of the statement follows now from Theorem 156 and the fact that the dual unit ball of a WLD Banach space, endowed with the $w^{*}$-topology, is a Corson compact space, hence angelic.

Remark 170 Banach spaces that are isomorphic to subspaces of $c_{0}\left(\omega_{1}\right)$ (resp. $\ell_{p}\left(\omega_{1}\right)$ for some $p \in(1,+\infty)$ ) are not necessarily isomorphic to $c_{0}\left(\omega_{1}\right)$ (resp. $\left.\ell_{p}\left(\omega_{1}\right)\right)$. It is enough to recall that $c_{0}$ has a subspace without a Schauder basis (Enflo, [Enf73]). However, the following simple result holds.

Corollary 171 Let $X$ be a Banach space generated by a subspace of $c_{0}\left(\omega_{1}\right)$ (resp. $\ell_{p}\left(\omega_{1}\right)$ for some $\left.p \in(1,+\infty)\right)$. Then $X$ is generated by $c_{0}\left(\omega_{1}\right)\left(\right.$ resp. $\left.\ell_{p}\left(\omega_{1}\right)\right)$.

Proof. It is enough to apply Corollary 157 and Proposition 151. 
The following intermediate result will be used in the proof of Theorem 158 .

Proposition 172 Let $X$ be a Banach space such that $\left(B_{X^{*}}, w^{*}\right)$ is angelic (more generally, $w^{*}$-sequentially compact). Then, for all $p \in(1,+\infty]$, every asymptotically p-flat set $M \subset X$ is an Asplund set.

Proof. We may assume that (5.5) holds in the original norm of $X$. Let $N \subset M$ be a countable set. Then, $\operatorname{span}_{\mathbb{Q}}(N)$, the set of all linear rational combinations of elements in $N$, is also countable. Let $Y:=\overline{\operatorname{span}}(N)$; this is a separable subspace. Let $q: X^{*} \rightarrow Y^{*}$ be the canonical quotient mapping. Given $y \in \operatorname{span}_{\mathbb{Q}}(N)$, find $\phi(y):=y^{*} \in S_{Y^{*}}$ such that $\left\langle y, y^{*}\right\rangle=\|y\|$. The Separation Theorem gives

$$
{\overline{\Gamma_{\mathbb{Q}}\left[\phi\left(\operatorname{span}_{\mathbb{Q}}(N)\right)\right]}}^{w\left(Y^{*}, Y\right)}=B_{Y^{*}},
$$

where $\Gamma_{\mathbb{Q}}$ denotes the absolutely rational-convex hull.

We shall prove that the countable set $\Gamma_{\mathbb{Q}}\left[\phi\left(\operatorname{span}_{\mathbb{Q}}(N)\right)\right]$ is $\|\cdot\|_{N}$-dense in $X^{*}$. This will conclude the proof.

To this end, choose $x^{*} \in X^{*}$. If $x^{*} \in Y^{\perp}$ we can find, as $\|\cdot\|$-close (in particular, as $\|\cdot\|_{N^{-}}$-close) to $x$ as we wish, an element which is not in $Y^{\perp}$. Hence, we may assume, without loss of generality, that $x^{*} \notin Y^{\perp}$. Assume, for the moment being, that $\left\|q x^{*}\right\|=1$. Let $y^{*}:=q x^{*}\left(\in S_{Y^{*}}\right)$. Since $Y$ is separable, $\left(B_{Y^{*}}, w^{*}\right)$ is metrizable, and so we can find a sequence $\left(y_{n}^{*}\right)$ in $\Gamma_{\mathbb{Q}}\left[\phi\left(\operatorname{span}_{\mathbb{Q}}(N)\right)\right]$ such that $y_{n}^{*} \stackrel{w^{*}}{\longrightarrow} y^{*}$. For each element $z^{*} \in \Gamma_{\mathbb{Q}}\left[\phi\left(\operatorname{span}_{\mathbb{Q}}(N)\right)\right]$, choose a single element $\psi\left(z^{*}\right)$ in $B_{X^{*}}$ such that $q\left(\psi\left(z^{*}\right)\right)=z^{*}$. Let $x_{n}^{*}:=\psi\left(y_{n}^{*}\right)$ for all $n \in \mathbb{N}$. The sequence $\left(x_{n}^{*}\right)$ has a $w^{*}$-cluster point $x_{0}^{*} \in B_{X^{*}}$, hence, by the assumption about the angelicity of $\left(B_{X^{*}}, w^{*}\right)$, there exists a subsequence of $\left(x_{n}^{*}\right)$ (denoted again $\left(x_{n}^{*}\right)$ ) such that $x_{n}^{*} \stackrel{w^{*}}{\longrightarrow} x_{0}^{*}$. Then we have

$$
\limsup _{n \rightarrow \infty}\left\|x_{n}^{*}\right\|^{q} \geq\left\|x_{0}^{*}\right\|^{q}+C \limsup _{n \rightarrow \infty}\left\|x_{0}^{*}-x_{n}^{*}\right\|_{M}^{q}
$$

Obviously, $q x_{n}^{*}=y_{n}^{*}\left(\stackrel{w^{*}}{\longrightarrow} y^{*}\right)$, hence $q x_{0}^{*}=y^{*}$, so $\left\|x_{0}^{*}\right\|=1$. It follows that $\limsup \sup _{n \rightarrow \infty}\left\|x_{n}^{*}\right\|^{q}=1$ and we get, from (5.26), that $\lim _{n \rightarrow \infty}\left\|x_{n}^{*}-x^{*}\right\|_{M}=0$. Hence, in particular, $\lim _{n \rightarrow \infty}\left\|x_{n}^{*}-x^{*}\right\|_{N}=0$. This proves the assertion for an element $x^{*} \in X^{*}$ such that $\left\|q x^{*}\right\|=1$, since the sequence $\left(x_{n}^{*}\right)$ is in the countable set $\psi\left[\Gamma_{\mathbb{Q}}\left[\phi\left(\operatorname{span}_{\mathbb{Q}}(N)\right)\right]\right]$. A homogeneity argument involving rational multiples of arbitrary elements in $X^{*}$ concludes the proof.

\section{Proof of Theorem 158}

(i) $\Rightarrow$ (ii). We shall follow almost word by word the proof of the implication (i) $\Rightarrow$ (ii) from Theorem 156, with the following changes. By Proposition 172, $M$ is an Asplund set. Then we can apply Proposition 166 for $M_{1}=M_{2}=\cdots=M$ and get a PRI $\left(P_{\alpha} ; \alpha \in\left[\omega_{0}, \omega_{1}\right]\right)$ on $(X,\|\cdot\|)$ such that $P_{\alpha}(M) \subset M$ for every $\alpha \in\left[\omega_{0}, \omega_{1}\right]$, and $\left\|P_{\lambda}^{*} f-P_{\alpha}^{*} f\right\|_{M} \rightarrow 0$ as $\alpha \uparrow \lambda$ whenever $f \in X^{*}$ and $\lambda \in\left(\omega_{0}, \omega_{1}\right]$ is a limit ordinal. We still have at hand the equality $\bigcup_{\omega_{0}<\alpha<\omega_{1}} P_{\alpha}^{*} X^{*}=X^{*}$. (It also follows from the angelicity of the dual unit ball provided with the $w^{*}$-topology.) 
Claims 1, 2, and 3 now read as:

Claim 1'. For every $0 \neq f \in X^{*}$, every $\varepsilon>0$, and every $\alpha \in\left[\omega_{0}, \omega_{1}\right)$ there is $\gamma_{f, \varepsilon, \alpha} \in\left(\alpha, \omega_{1}\right)$ such that

$$
\|f+g\|_{M}^{q} \geq(1-\varepsilon)\|f\|_{M}^{q}+C\|g\|_{M}^{q} \quad \text { whenever } \quad g \in \operatorname{ker} P_{\gamma_{f, \varepsilon, \alpha}^{*}}^{*} \quad \text { and } \quad\|g\|<\frac{1}{\varepsilon} .
$$

Claim 2'. For every $\alpha \in\left[\omega_{0}, \omega_{1}\right)$ there exists $\beta_{\alpha} \in\left(\alpha, \omega_{1}\right)$ such that

$$
\|f+g\|_{M}^{q} \geq\|f\|_{M}^{q}+C\|g\|_{M}^{q} \quad \text { whenever } f \in P_{\alpha}^{*} X^{*} \quad \text { and } \quad g \in \operatorname{Ker} P_{\beta_{\alpha}}^{*} .
$$

Claim 3'. There exists an increasing long sequence $\left(\delta_{\alpha}\right)_{\omega_{0} \leq \alpha \leq \omega_{1}}$ in $\left[\omega_{0}, \omega_{1}\right]$, with $\delta_{\omega_{0}}:=\omega_{0}$ and $\delta_{\omega_{1}}:=\omega_{1}$, and such that for every $\alpha \in\left[\omega_{0}, \omega_{1}\right)$ we have

$$
\|f+g\|_{M}^{q} \geq\|f\|_{M}^{q}+C\|g\|_{M}^{q} \quad \text { whenever } \quad f \in P_{\delta_{\alpha}}^{*} X^{*} \quad \text { and } g \in \operatorname{ker} P_{\delta_{\alpha}}^{*} .
$$

The proofs of Claims 1', 2' and 3' follow the corresponding proofs of Claims 1, 2, and 3 with the change that the norm $\|\cdot\|$ in $X$ should be everywhere replaced by the seminorm $\|\cdot\|_{M}$. Moreover, in Claim 2', we use the fact that, once $M$ is and Asplund set, then $P_{\alpha}^{*} X^{*}, \omega_{0} \leq \alpha<\omega_{1}$, are separable spaces in the metric coming from the seminorm $\|\cdot\|_{M}$

The statement of Claim 4' is the same as that of Claim 4. In its proof, we profit from the inequality $\|\cdot\|_{M} \leq\|\cdot\|$ and from the fact that the properties of $P_{\alpha}^{*}$ 's guarantee that $Y$ is dense in $X^{*}$ in the metric coming from $\|\cdot\|_{M}$.

The rest of the proof is the same as in the proof of Theorem 156 .

(ii) $\Rightarrow$ (i). Take $p \in(1, \infty)$. Assume there exists a bounded linear operator $S$ : $\ell_{p}\left(\omega_{1}\right) \rightarrow X$, with dense range. Put $q=\frac{p}{p-1}$ and $M=S\left(B_{\ell_{p}\left(\omega_{1}\right)}\right)$. Then $S^{*}$ : $X^{*} \rightarrow \ell_{q}\left(\omega_{1}\right)$ is an injection, and hence the space $X$ is weakly Lindelöf determined. Let $f \in X^{*}$ and consider a $w^{*}$-null sequence $\left(f_{n}\right)$ in $X^{*}$. Then $S^{*} f_{n} \rightarrow 0$ in the $w^{*}$-topology, and hence

$$
\begin{aligned}
& \limsup _{n \rightarrow \infty}\left\|f+f_{n}\right\|_{M}^{q}=\limsup _{n \rightarrow \infty} \sup \left\langle M, f+f_{n}\right\rangle^{q} \\
& \quad=\limsup _{n \rightarrow \infty} \sup \left\langle B_{\ell_{p}\left(\omega_{1}\right)}, S^{*} f+S^{*} f_{n}\right\rangle^{q} \\
& \quad=\limsup _{n \rightarrow \infty}\left\|S^{*} f+S^{*} f_{n}\right\|_{\ell_{q}}^{q} \geq\left\|S^{*} f\right\|_{\ell_{q}}^{q}+\limsup _{n \rightarrow \infty}\left\|S^{*} f_{n}\right\|_{\ell_{q}}^{q} \\
& \quad=\|f\|_{M}^{q}+\limsup _{n \rightarrow \infty}\left\|f_{n}\right\|_{M}^{q} .
\end{aligned}
$$

This shows that the set $M$ is innerly asymptotically $p$-flat.

The case of inner asymptotical $\infty$-flatness can be dealt with analogously. 


\subsection{Some remarks on long Schauder bases}

This section is purely instrumental. The theory of long Schauder bases appears fragmentarily in [BP75] and in [HMVZ07, Chapter 4]. We need some extra results, none of them surprising since they mimic the well-known behavior of the standard countable Schauder bases. However, some arguments are delicate and we think it is worth to provide them in detail.

As it is usual, we shall identify an interval of ordinal with the "next" ordinal. Precisely, given an ordinal $\Gamma$, we shall identify $[0, \Gamma)$ with $\Gamma$, if there is no possibility of misunderstanding. So, we shall write indistinctly $0 \leq \gamma<\Gamma$ or $\gamma \in \Gamma$.

We start by recalling here some definitions and some simple facts.

Let $\Gamma$ be an infinite ordinal. A subset $\left\{x_{\gamma} ; \gamma \in \Gamma\right\}$ of $X$ will be called a long sequence in $X$. We shall use the notation $\left(x_{\gamma}\right)_{\gamma \in \Gamma}$ (or just $\left(x_{\gamma}\right)_{\Gamma}$, or even $\left(x_{\gamma}\right)$ if the index set is understood) for a long sequence.

Definition 173 Let $(X,\|\cdot\|)$ be a normed space. Let $\Gamma$ be an infinite ordinal. Endow $\Gamma$ with the order topology. Given a long sequence $\left(x_{\gamma}\right)_{\gamma \in \Gamma}$ in $X$, we shall say that the sum $\sum_{\gamma \in \Gamma} x_{\gamma}$ is well defined and sums to $x \in X$ if there exits a continuous mapping $S:[0, \Gamma] \rightarrow X$ such that

(i) $S(0)=0$,

(ii) $S(\gamma+1)=S(\gamma)+x_{\gamma}$ for every $\gamma \in[0, \Gamma)$, and

(iii) $S(\Gamma)=x$.

If this is the case, then the symbol $\sum_{\gamma \in \Gamma} x_{\gamma}$ also means the vector $x$.

Of course, this definition coincides with the usual definition of the sum of a sequence when the index set is $\Gamma:=\omega_{0}$.

The following proposition is almost obvious. However, we collect this result here for later reference.

Proposition 174 Let $X$ and $Y$ be normed spaces and let $T: X \rightarrow Y$ be a continuous linear mapping. Let $\left(x_{\gamma}\right)_{\gamma \in \Gamma}$ be a long sequence in $X$ that sums to $x \in X$. Then $\left(T x_{\gamma}\right)_{\gamma \in \Gamma}$ sums to $T x$.

Proof. Let $S: \Gamma \rightarrow X$ be the continuous mapping introduced in Definition 173 . Then the mapping $T \circ S: \Gamma \rightarrow Y$ is continuous, and we have the following.

(i) $T \circ S(0)=T 0=0$,

(ii) $T \circ S(\gamma+1)=T(S(\gamma+1))=T\left(S(\gamma)+x_{\gamma}\right)=T \circ S(\gamma)+T x_{\gamma}$, for all $\gamma \in[0, \Gamma)$, and

(iii) $T \circ S(\Gamma)=T x$.

Then, according to Definition 173 , the long sequence $\left(T x_{\gamma}\right)_{\gamma \in \Gamma}$ sums to $T x$.

Definition $175 \operatorname{Let}(X,\|\cdot\|)$ be a normed space. A long sequence $\left(x_{\gamma}\right)_{\gamma \in \Gamma}$ of vectors is called a long Schauder basis if, for every $x \in X$, there exists a unique long sequence of scalars $\left(a_{\gamma}\right)_{\gamma \in \Gamma}$ such that $\sum_{\gamma \in \Gamma} a_{\gamma} x_{\gamma}$ sums to $x$. It is called a long Schauder basic sequence if it is a long Schauder basis of the space $\overline{\operatorname{span}}\left\{x_{\gamma} ; \gamma \in \Gamma\right\}$. 
Again, a long Schauder basis, if $\Gamma=\omega_{0}$, turns out to be a classical Schauder basis.

Given a long Schauder basis $\left(x_{\gamma}\right)_{\gamma \in \Gamma}$ in $X$, and given $x \in X$, the long sequence $\left(a_{\gamma} x_{\gamma}\right)_{\gamma \in \Gamma}$ in Definition 175 sums to $x$ in the sense of Definition 173, so there exists a mapping $S(\cdot, x):[0, \Gamma] \rightarrow X$ with the properties there. We shall use the following notation. Given $x \in X$ and $0 \leq \gamma \leq \Gamma$, put $S_{\gamma}(x):=S_{x}(\gamma):=S(\gamma, x)\left(=\sum_{\alpha \in \gamma} a_{\alpha} x_{\alpha}\right)$.

In [BP75], the following notion is introduced ${ }^{1}$.

Definition 176 Let $(X,\|\cdot\|)$ be a normed space. Let $\Gamma$ be an infinite ordinal number. A projection basis of type $\Gamma$ is a long sequence $\left(P_{\gamma}\right)_{0 \leq \gamma \leq \Gamma}$ of continuous linear projections on $X$ such that:

(i) For every $x \in X$, the mapping $\gamma \mapsto P_{\gamma}(x)$ from $[0, \Gamma]$ into $X$, is continuous.

(ii) $P_{0}(x)=0, P_{\Gamma}(x)=x, P_{\alpha} P_{\beta}=P_{\min \{\alpha, \beta\}}$ for all $\alpha, \beta \in[0, \Gamma]$ and $x \in X$.

(iii) For every $\gamma \in[0, \Gamma)$ the space $\left(P_{\gamma+1}-P_{\gamma}\right) X$ is 1-dimensional.

We shall prove that the existence of a long Schauder basis $\left(x_{\gamma}\right)_{\gamma \in \Gamma}$ is equivalent to the existence of a projection basis of type $\Gamma$.

Definition 177 Let $(X,\|\cdot\|)$ be a normed space. Given a long Schauder basis $\left(x_{\gamma}\right)_{\gamma \in \Gamma}$ in $X$ and a projection basis $\left(P_{\gamma}\right)_{0 \leq \gamma \leq \Gamma}$ of type $\Gamma$, we shall say that they are associated whenever $\left(P_{\gamma+1}-P_{\gamma}\right) X=\operatorname{span}\left\{x_{\gamma}\right\}$ for every $\gamma \in \Gamma$.

Proposition 178 Let $(X,\|\cdot\|)$ be a normed space. Then, given a long Schauder basis $\left(x_{\gamma}\right)_{\gamma \in \Gamma}$ in $X$, there exists a projection basis $\left(P_{\gamma}\right)_{0 \leq \gamma \leq \Gamma}$ of type $\Gamma$ associated to

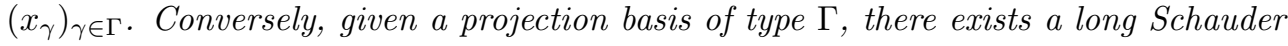
basis associated to it.

Proof. Given a long Schauder basis $\left(x_{\gamma}\right)_{\gamma \in \Gamma}$ in $X$, the long sequence $\left(S_{\gamma}\right)_{0 \leq \gamma \leq \Gamma}$ is a projection basis of type $\Gamma$ associated to $\left(x_{\gamma}\right)_{\gamma \in \Gamma}$, as it is easy to check from the definition.

Conversely, let $\left(P_{\gamma}\right)_{0 \leq \gamma \leq \Gamma}$ be a projection basis of type $\Gamma$ in $X$. Then, if $\left(P_{\gamma+1}-\right.$ $\left.P_{\gamma}\right) X=\operatorname{span}\left\{x_{\gamma}\right\}$ for every $\gamma \in \Gamma$, the long sequence $\left(x_{\gamma}\right)_{\gamma \in \Gamma}$ is a long Schauder basis in $X$ associated to $\left(P_{\gamma}\right)_{0 \leq \gamma \leq \Gamma}$. Indeed, given $x \in X$ and $0 \leq \gamma<\Gamma$, put $\left(P_{\gamma+1}-P_{\gamma}\right) x=a_{\gamma} x_{\gamma}$ for some scalar $a_{\gamma}$ (uniquely defined). It is enough to prove that the long sequence $\left(a_{\gamma} x_{\gamma}\right)_{\gamma \in \Gamma}$ sums to $x$. This is a consequence of the fact that the mapping $S_{x}:[0, \Gamma] \rightarrow X$ needed in Definition 173 for summing $\left(a_{\gamma} x_{\gamma}\right)_{\gamma \in \Gamma}$ is just $P_{(.)} x$. This can be easily proved by induction using the properties of the projection basis.

So, from now on, given a long Schauder basis $\left(x_{\gamma}\right)_{\gamma \in \Gamma}$ or, alternatively, a projection basis $\left(P_{\gamma}\right)_{0 \leq \gamma \leq \Gamma}$ of type $\Gamma$, we shall use the following relationships: $S_{\gamma}=P_{\gamma}, P_{\gamma}(x)=$ $\sum_{\alpha \in \gamma} a_{\alpha} x_{\alpha}$ whenever $x:=\sum_{\gamma \in \Gamma} a_{\gamma} x_{\gamma}$, for all $0 \leq \gamma \leq \Gamma$, and $\left(P_{\gamma+1}-P_{\gamma}\right) x=a_{\gamma} x_{\gamma}$, for all for all $0 \leq \gamma<\Gamma$. We call $\left(P_{\gamma}\right)_{0 \leq \gamma \leq \Gamma}$, from now on, the long sequence of projections associated to the long Schauder basis $\left(x_{\gamma}\right)_{\gamma \in \Gamma}$.

\footnotetext{
${ }^{1}$ Indeed, we adapt the definition given there to Definition 176 , allowing some change in the notation in order to fit precisely the schema provided above.
} 
Proposition 179 Let $\left(x_{\gamma}\right)_{\gamma \in \Gamma}$ be a long sequence in a Banach space $X$. Then, the following are equivalent.

(i) $\left(x_{\gamma}\right)_{\gamma \in \Gamma}$ is a long Schauder basic sequence.

(ii) There exists a constant $C>0{ }^{2}$ such that, for every $n \in \mathbb{N}$, every $\gamma_{1}<\gamma_{2}<\ldots<$ $\gamma_{n}$ in $\Gamma$ and every $a_{1}, a_{2}, \ldots, a_{n} \in \mathbb{R}$, we have

$$
\left\|\sum_{i=1}^{k} a_{i} x_{\gamma_{i}}\right\| \leq C\left\|\sum_{i=1}^{n} a_{i} x_{\gamma_{i}}\right\| \quad \text { for all } 1 \leq k \leq n
$$

Proof. Assume that (i) holds. Let $Y:=\overline{\operatorname{span}}\left\{x_{\gamma} ; \gamma \in \Gamma\right\}$. Then $\left(x_{\gamma}\right)_{\gamma \in \Gamma}$ is a long Schauder basis of $Y$. Because of [HMVZ07, Theorem 4.6], there exists $C>0$ such that $\left\|P_{\gamma}\right\| \leq C$, for all $0 \leq \gamma \leq \Gamma$. Then, for a finite set $\left\{\gamma_{1}<\gamma_{2}<\ldots<\gamma_{n}\right\} \subset \Gamma$ and scalars $\left\{a_{k} ; k=1,2, \ldots\right\}$, we have

$$
\left\|\sum_{i=1}^{k} a_{i} x_{\gamma_{i}}\right\|=\left\|P_{\gamma_{k}+1}\left(\sum_{i=1}^{n} a_{i} x_{\gamma_{i}}\right)\right\| \leq C\left\|\sum_{i=1}^{n} a_{i} x_{\gamma_{i}}\right\| \quad \text { for all } \quad 1 \leq k \leq n,
$$

which is (ii).

Assume now that (ii) holds. Let $Y_{0}:=\operatorname{span}\left\{x_{\gamma} ; \gamma \in \Gamma\right\}$. We shall define mappings $P_{\gamma}: Y_{0} \rightarrow Y_{0}$ for all $0 \leq \gamma \leq \Gamma$. Fix $\gamma \in \Gamma$. Given $x \in Y_{0}$, we have $x:=\sum_{i=1}^{n} a_{i} x_{\gamma_{i}}$ for some $n \in \mathbb{N}$, some $\gamma_{1}<\gamma_{2}<\ldots<\gamma_{n}$ and some scalars $a_{1}, a_{2}, \ldots, a_{n}$. Define $P_{\gamma} x:=\sum_{i=1}^{k} a_{i} x_{\gamma_{i}}$, whenever $\left\{\gamma_{i} ; i=1,2, \ldots, n\right\} \cap[0, \gamma)=\left\{\gamma_{i} ; i=1,2, \ldots, k\right\}$. If the set $\left\{\gamma_{i} ; i=1,2, \ldots, n\right\} \cap[0, \gamma)$ is empty, we put $P_{\gamma} x=0$. Define $P_{\Gamma}:=\operatorname{Id}_{\mathrm{X}}$. Then, it follows from (5.27) that $\left\|P_{\gamma} x\right\| \leq C\|x\|$ for all $0 \leq \gamma \leq \Gamma$, and it is clear that $\left(P_{\gamma}\right)_{0 \leq \gamma \leq \Gamma}$ satisfies (i), (ii) and (iii) in Definition 176. So $\left(P_{\gamma}\right)_{0 \leq \gamma \leq \Gamma}$ is the family of canonical projections associated to some long Schauder basis of $Y$, and $\left\|P_{\gamma}\right\| \leq C$ for all $0 \leq \gamma \leq \Gamma$. Obviously, $\left(x_{\gamma}\right)_{\gamma \in \Gamma}$ is the corresponding long Schauder basis of $Y$. It suffices to apply the fact that a long Schauder basis of a normed space with uniformly bounded associated projection basis is a long Schauder basis of its completion (see [HMVZ07, Fact 4.4]) to finish the proof.

A standard transfinite argument gives the following result, that reduces the summation needed for representing a particular element in a space with a long Schauder basis to a countable sum.

Proposition 180 ([HMVZ07], Fact 4.7) Let $X$ be a normed space and $\left(x_{\gamma}\right)_{\gamma \in \Gamma}$ a long Schauder basis of $X$. If $\sum_{\gamma \in \Gamma} a_{\gamma} x_{\gamma}$ sums to $x \in X$, then there exists a sequence $\left(\gamma_{n}\right)_{n \in \mathbb{N}}$ such that $\left\{\gamma \in \Gamma ; a_{\gamma} \neq 0\right\}=\left\{\gamma_{1}, \gamma_{2}, \ldots\right\}$ and $x=\sum_{n=1}^{\infty} a_{\gamma_{n}} x_{\gamma_{n}}$.

As in the countable case we can introduce the following concept.

Definition 181 Let $X$ and $Y$ be normed spaces. Let $\left(x_{\gamma}\right)_{\gamma \in \Gamma}$ and $\left(y_{\gamma}\right)_{\gamma \in \Gamma}$ be two long Schauder basic sequences, in $X$ and in $Y^{*}$, respectively. We say that $\left(x_{\gamma}\right)_{\gamma \in \Gamma}$ is

\footnotetext{
${ }^{2}$ The smallest constant $C$ in $(5.27)$ is called the basis constant associated to the long Schauder basic sequence.
} 
equivalent to $\left(y_{\gamma}\right)_{\gamma \in \Gamma}$ if for every long sequence $\left(a_{\gamma}\right)_{\gamma \in \Gamma}$ of scalars $\sum_{\gamma \in \Gamma} a_{\gamma} x_{\gamma}$ sums to a vector if and only if $\sum_{\gamma \in \Gamma} a_{\gamma} y_{\gamma}$ sums to a vector.

Proposition 182 Let $X, Y$ be Banach spaces. Let $\left(x_{\gamma}\right)_{\gamma \in \Gamma}$ and $\left(y_{\gamma}\right)_{\gamma \in \Gamma}$ be two long sequences, in $X$ and in $Y$, respectively. Assume that $\left(x_{\gamma}\right)_{\gamma \in \Gamma}$ is a long Schauder basic sequence. Then the following statements are equivalent.

(i) $\left(y_{\gamma}\right)_{\gamma \in \Gamma}$ is a long Schauder basic sequence equivalent to $\left(x_{\gamma}\right)_{\gamma \in \Gamma}$.

(ii) There exists an isomorphism $T: \overline{\operatorname{span}}\left\{x_{\gamma} ; \gamma \in \Gamma\right\} \rightarrow \overline{\operatorname{span}}\left\{y_{\gamma} ; \gamma \in \Gamma\right\}$ such that $T x_{\gamma}=y_{\gamma}$ for every $\gamma \in \Gamma$.

(iii) There exist positive constants $A$ and $B$ such that, for every $n \in \mathbb{N}$, every $\gamma_{1}, \gamma_{2}, \ldots, \gamma_{n} \in \Gamma$, and every $b_{1}, b_{2}, \ldots, b_{n} \in \mathbb{R}$

$$
A\left\|\sum_{k=1}^{n} b_{k} x_{\gamma_{k}}\right\| \leq\left\|\sum_{k=1}^{n} b_{k} y_{\gamma_{k}}\right\| \leq B\left\|\sum_{k=1}^{n} b_{k} x_{\gamma_{k}}\right\|
$$

Proof. The proof is an adaptation of the classical proof for countable Schauder basic sequences. We shall provide the details for the sake of completeness.

(i) $\Rightarrow$ (ii). Put $X_{0}:=\operatorname{span}\left\{x_{\gamma} ; \gamma \in \Gamma\right\}$ and $Y_{0}:=\operatorname{span}\left\{y_{\gamma} ; \gamma \in \Gamma\right\}$. We define a mapping $T: \bar{X}_{0} \rightarrow \bar{Y}_{0}$ by the formula

$$
T\left(\sum_{\gamma \in \Gamma} a_{\gamma} x_{\gamma}\right)=\sum_{\gamma \in \Gamma} a_{\gamma} y_{\gamma}
$$

whenever $\sum_{\gamma \in \Gamma} a_{\gamma} x_{\gamma}$ exists. The definition is consistent since both long Schauder basic sequences are equivalent and, obviously, $\left(x_{\gamma}\right)_{\gamma \in \Gamma}$ is a long Schauder basis of $X_{0}$. $T$ is clearly a linear mapping. We shall prove that it has closed graph. Assume that $\left(u_{n}, v_{n}\right)_{n \in \mathbb{N}}$ is a sequence in Graph $T$ that converges to some $\left(u_{0}, v_{0}\right) \in \bar{X}_{0} \times \bar{Y}_{0}$. For $n \in \mathbb{N} \cup\{0\}$, put $u_{n}:=\sum_{\gamma \in \Gamma} a_{\gamma}^{n} x_{\gamma}$; then $v_{n}=\sum_{\gamma \in \Gamma} a_{\gamma}^{n} y_{\gamma}$. Since the canonical projections associated to the long basic Schauder sequences are continuous (see Proposition 178), it follows that $\lim _{n \rightarrow \infty} a_{\gamma}^{n}=a_{\gamma}^{0}$, for every $\gamma \in \Gamma$. Then $\left(u_{0}, v_{0}\right) \in \operatorname{Graph} T$. From the Closed Graph Theorem, $T$ is continuous. It is one-to-one, due to the fact that $\left(y_{\gamma}\right)_{\gamma \in \Gamma}$ is a long Schauder basic sequence. In order to see that it is an isomorphism, it remains to show that $T$ is onto. This is due, again, to the fact that $\left(y_{\gamma}\right)_{\gamma \in \Gamma}$ is a long Schauder basis of $\bar{Y}_{0}$.

(ii) $\Rightarrow$ (iii) is obvious.

(iii) $\Rightarrow\left(\right.$ i). Take any set $\left\{\gamma_{i} ; i=1,2, \ldots, n\right\} \subset \Gamma$ and any scalars $\left\{a_{i} ; i=1,2, \ldots, n\right\}$. Let $k \in\{1,2, \ldots, n\}$. Then, it follows from Proposition 179 applied to $\left(x_{\gamma}\right)_{\gamma \in \Gamma}$ and (iii) that,

$$
\left\|\sum_{i=1}^{k} b_{i} y_{\gamma_{i}}\right\| \leq B\left\|\sum_{i=1}^{k} b_{i} x_{\gamma_{i}}\right\| \leq B C\left\|\sum_{i=1}^{n} b_{i} x_{\gamma_{i}}\right\| \leq A B C\left\|\sum_{i=1}^{n} b_{i} y_{\gamma_{i}}\right\|,
$$

where $C$ is the basis constant associated to $\left(x_{\gamma}\right)_{\gamma \in \Gamma}$. By Proposition 179 we get that $\left(y_{\gamma}\right)_{\gamma \in \Gamma}$ is a long Schauder basic sequence. Let $X_{0}$ and $Y_{0}$ be the normed spaces 
defined in the proof of (i) $\Rightarrow\left(\right.$ ii) above. Define $T_{0}: X_{0} \rightarrow Y_{0}$ by $T_{0}\left(\sum_{i=1}^{n} a_{i} x_{\gamma_{i}}\right)=$ $\sum_{i=1}^{n} a_{i} y_{\gamma_{i}}$, where $n \in \mathbb{N},\left\{\gamma_{i} ; i=1,2, \ldots, n\right\} \subset \Gamma$ and $\left\{a_{i} ; i=1,2, \ldots, n\right\} \subset \mathbb{R}$. Let $S_{0}: Y_{0} \rightarrow X_{0}$ be the inverse mapping. Both mappings are linear, and according to (iii), both are continuous. Then both are isomorphisms, and they have unique extensions $T$ to $\bar{X}_{0}$ and $S$ to $\bar{Y}_{0}$, respectively. Since $S \circ T$ is the (unique) extension to $\bar{X}_{0}$ of $S_{0} \circ T_{0}\left(=\operatorname{Id}_{X_{0}}\right)$, it follows that $T$ (and $S$ ) is an isomorphism, and $T x_{\gamma}=y_{\gamma}$ for all $\gamma$. This is (ii).

Let $\Gamma$ be a non-empty set. Given $v \in \mathbb{R}^{\Gamma}$, let supp $v:=\{\gamma \in \Gamma ; v(\gamma) \neq 0\}$.

Proposition 183 Let $\left(x_{\gamma}\right)_{\gamma \in \Gamma}$ be a long sequence of vectors in $c_{0}(I)$ for some nonempty set $I$. Assume that there exists $0<A \leq B$ such that $0<A \leq\left\|x_{\gamma}\right\|_{\infty} \leq B$ for all $\gamma \in \Gamma$ and $\operatorname{supp} x_{\gamma} \cap \operatorname{supp} x_{\delta}=\emptyset$ for all distinct $\gamma, \delta \in \Gamma$. Then $\left(x_{\gamma}\right)_{\gamma \in \Gamma}$ is a long basic sequence equivalent to the canonical basis of $c_{0}(\Gamma)$.

Proof. Let $\left(e_{i}\right)_{i \in I}$ be the canonical basis of $c_{0}(I)$. We shall prove that, given $n \in$ $\mathbb{N}, \gamma_{1}, \ldots, \gamma_{n} \in \Gamma$, and scalars $a_{1}, \ldots, a_{n}$, we have

$$
A\left\|\sum_{k=1}^{n} a_{k} e_{\gamma_{k}}\right\|_{\infty} \leq\left\|\sum_{k=1}^{n} a_{k} x_{\gamma_{k}}\right\|_{\infty} \leq B\left\|\sum_{k=1}^{n} a_{k} e_{\gamma_{k}}\right\|_{\infty} .
$$

Then, it will be enough to apply Proposition 182 to get the conclusion. It is obvious that, due to the disjointness of the supports,

$$
\begin{aligned}
& A \cdot \sup \left\{\left|a_{k}\right| ; 1 \leq k \leq n\right\} \leq\left\|\sum_{k=1}^{n} a_{k} x_{i_{k}}\right\|_{\infty} \\
& \quad=\sup \left\{\left\|a_{k} x_{i_{k}}\right\|_{\infty} ; 1 \leq k \leq n\right\} \leq B \cdot \sup \left\{\left|a_{k}\right| ; 1 \leq k \leq n\right\},
\end{aligned}
$$

and this is (5.29).

Proposition 184 Let $X$ be a Banach space and let $T: c_{0}(I) \rightarrow X$ be a bounded linear operator. Assume that $\left(v_{\gamma}\right)_{\gamma \in \Gamma}$ is a long basic sequence in $c_{0}(I)$ equivalent to the canonical basis $\left(e_{\gamma}\right)_{\gamma \in \Gamma}$ of $c_{0}(\Gamma)$. Assume, too, that there exists $K>0$ such that $\left\|T v_{\gamma}\right\| \geq K$ for all $\gamma \in \Gamma$ and that $\left(T v_{\gamma}\right)_{\gamma \in \Gamma}$ is a basic sequence. Then $\left(T v_{\gamma}\right)_{\gamma \in \Gamma}$ is equivalent to $\left(v_{\gamma}\right)_{\gamma \in \Gamma}$, and so to the canonical basis of $c_{0}(\Gamma)$.

Proof. Let $\left(b_{\gamma}\right)_{\gamma \in \Gamma}$ a sequence of scalars such that $\sum_{\gamma \in \Gamma} b_{\gamma} v_{\gamma}$ converges (to some $\left.z \in c_{0}(\Gamma)\right)$. Then, since $T$ is continuous, Proposition 174 says that $\sum_{\gamma \in \Gamma} b_{\gamma} T v_{\gamma}$ converges (to $T z$ ).

Conversely, assume that $\sum_{\gamma \in \Gamma} b_{\gamma} T v_{\gamma}$ converges (to some $x \in X$ ). By Proposition 180 , there exists a reordering of the set $\left\{\gamma \in \Gamma ; b_{\gamma} \neq 0\right\}$ into a sequence $\left(\gamma_{n}\right)_{n \in \mathbb{N}}$ so that $x=\sum_{n=1}^{\infty} b_{\gamma_{n}} T v_{\gamma_{n}}$. Then, $\left\|b_{\gamma_{n}} T v_{\gamma_{n}}\right\| \rightarrow 0$ (we are dealing with a classical countable sum), so we have

$$
\left|b_{\gamma_{n}}\right|=\left\|b_{\gamma_{n}} T v_{\gamma_{n}}\right\| \frac{1}{\left\|T v_{\gamma_{n}}\right\|} \leq\left\|b_{\gamma_{n}} T v_{\gamma_{n}}\right\| \frac{1}{K} \rightarrow 0 \quad \text { as } \quad n \rightarrow \infty .
$$


This means that the long sequence $\left(b_{\gamma}\right)_{\gamma \in \Gamma}$ belongs to $c_{0}(\Gamma)$. It follows that $\sum_{\gamma \in \Gamma} b_{\gamma} e_{\gamma}$ converges, and so $\sum_{\gamma \in \Gamma} b_{\gamma} v_{\gamma}$ converges, too. This proves the assertion.

\subsection{Fixing $c_{0}(\Gamma)$ by operators}

\subsubsection{Introduction and results}

Definition 185 We say that a bounded linear operator $T$ from a Banach space $X$ into a Banach space $Y$ fixes a copy of a subspace $Z$ of $X$ if the restriction $T\lceil Z$ is an isomorphism on $Z$

In particular, if this is the case, the space $Y$ has an isomorphic copy of $Z$. It has been an area of continued interest to know whether a given Banach space has isomorphic copies of classical spaces (spaces of functions, spaces of sequences, etc.). Quite easily, in many cases, it is possible to produce a continuous linear operator from one of this "classical" spaces into the given Banach space (see, for example, [DGZ, Chapter VI, Section 5 and 6] or [Di84, Chapter 11], by taking adjoint mappings), so a particular technique for investigating the basic problem of the existence of isomorphic copies consists in "selecting" a subspace where the operator acts as an isomorphism, because it is not uncommon that subspaces of classical spaces are isomorphic to the whole space.

As an example of what has been said in the former paragraph let us consider the following results.

Theorem 186 (Dunford, Pettis, Pełczyński, see e.g. [DiUh77]) Let $X$ be a Banach space. Let $T: c_{0}(\mathbb{N}) \rightarrow X$ be a bounded linear and non-weakly compact operator. Then $T$ fixes a copy of $c_{0}(\mathbb{N})$.

Remark 187 Observe that, in the case considered in Theorem 186, the class of $\|\cdot\|$-compact operators coincide with the class of $w$-compact operators. Indeed, every $\|\cdot\|$-compact operator is $w$-compact. Assume now that $T: c_{0}(\Gamma) \rightarrow X$ is $w$-compact. The Gantmacher theorem (see, e.g., [FHHMPZ01, Theorem 11.28]) says that $T^{*}$ : $X^{*} \rightarrow \ell_{1}(\Gamma)$ is $w$-compact. Since $\ell_{1}(\Gamma)$ has the Schur property, $T^{*}$ is norm-compact. Finnally by Gantmacher's theorem, $T$ is norm-compact. The conclusion follows from the Schauder theorem (see, e.g. [FHHMPZ01, Theorem 7.7]).

A companion to the former result is the following theorem, due to Rosenthal.

Theorem 188 (Rosenthal, [R70]) Let $T: c_{0}(\Gamma) \rightarrow X$ be a bounded linear operator such that, for some $\varepsilon>0,\left\|T\left(e_{\gamma}\right)\right\|>\varepsilon$ for every $\gamma \in \Gamma$. Then there exists $\Gamma^{\prime} \subset \Gamma$ of the same cardinality as $\Gamma$ such that $T$ restricted to $c_{0}\left(\Gamma^{\prime}\right)$ is an isomorphism. 
Remark 189 Theorem 188 has recently been improved in [ACGJM] in the following way. Let $T: c_{0}(\Gamma) \rightarrow X$ be a bounded linear operator such that, for some $\varepsilon>0$, $\left\|T\left(e_{\gamma}\right)\right\| \geq \varepsilon$ for all $\gamma \in \Gamma$, where $e_{\gamma}$ 's are the canonical unit vectors in $c_{0}(\Gamma)$. Then there exists a finite partition $\left\{\Gamma_{1}, \ldots, \Gamma_{n}\right\}$ of $\Gamma$ such that the operator $T_{\left\lceil c_{0}\left(\Gamma_{k}\right)\right.}$ is an isomorphism for every $k=1,2, \ldots, n$.

Remark 190 Operators appearing in Theorem 188 are not $\|\cdot\|$-compact (hence, not weakly compact, see Remark 187). Indeed, assume that such a $T$ is $\|\cdot\|$-compact. Then $T\left(B_{c_{0}(\Gamma)}\right)$ is a $\|\cdot\|$-relatively compact subset of $X$. Let $\left(\gamma_{n}\right)$ be a sequence of distinct elements in $\Gamma$. Then, $e_{\gamma} \stackrel{w}{\longrightarrow} 0$. Since $T$ is weak-to-weak continuous, we get $T e_{\gamma} \stackrel{w}{\longrightarrow} 0$. On $\left(B_{c_{0}(\Gamma)}\right)$, the weak and norm topologies coincide, so we get $T e_{\gamma} \stackrel{\|\cdot\|}{\longrightarrow} 0$, a contradiction with the fact that $\left\|T e_{\gamma}\right\| \geq \varepsilon$ for all $\gamma \in \Gamma$.

The next result is the $\ell_{\infty}(\Gamma)$-version of Theorem 188 .

Theorem 191 (Rosenthal, [R70]) Let $X$ be a Banach space. Assume $T: \ell_{\infty}(\Gamma) \rightarrow$ $X$ is such that $\inf _{\gamma \in \Gamma}\left\|T\left(e_{\gamma}\right)\right\|>0$ (where $e_{\gamma}$ is the $\gamma$ th unit vector in $\ell_{\infty}(\Gamma)$ ). Then there exists a set $\Gamma^{\prime} \subset \Gamma$, with $\# \Gamma^{\prime}=\# \Gamma$, such that $T_{\mid \ell_{\infty}\left(\Gamma^{\prime}\right)}$ is an isomorphism. This holds, in particular, if $T_{\left\lceil c_{0}(\Gamma)\right.}$ is an isomorphism.

The following two corollaries are worth to mention.

Corollary 192 Let $X$ be a Banach space generated by $c_{0}\left(\omega_{1}\right)$. Then, any bounded linear operator from $c_{0}\left(\omega_{1}\right)$ onto a dense set in $X$ fixes a copy of $c_{0}\left(\omega_{1}\right)$.

Corollary 193 (i) Let $X$ be an Asplund Banach space of density $\omega_{1}$ generated by an asymptotically $\infty$-flat set. Then $X$ contains an isomorphic copy of $c_{0}\left(\omega_{1}\right)$.

(ii) Let $X$ be a Banach space of density $\omega_{1}$ generated by an innerly asymptotically $\infty$-flat set. Then $X$ contains an isomorphic copy of $c_{0}\left(\omega_{1}\right)$.

\subsubsection{Proofs}

As a byproduct of the work done in previous sections, we get simple proofs of Theorems 186 and 188 that use techniques from the theory of projectional resolutions of the identity only.

Let $\Gamma$ be an infinite set. Given $v \in \mathbb{R}^{\Gamma}$, let $\operatorname{supp} v$ be the support of $v$. Let $c_{00}(\Gamma)$ be the space of all finitely supported vectors in $\mathbb{R}^{\Gamma}$.

The proof of Theorem 186 provided below seems to be new. It is simpler than the original one (see [DiUh77]).

Proof of Theorem 186. The set $T\left(B_{c_{0}(\mathbb{N})}\right)$ is not precompact. Then, there exists $\varepsilon>0$ such that

$$
T\left(B_{c_{0}(\mathbb{N})}\right) \text { cannot be covered by a finite number of } \varepsilon \text {-small sets. }
$$


Since $T$ is continuous, there exists $0<\delta<1$ such that $\|T(w)\| \leq \varepsilon / 2$ whenever $w \in c_{0}(\Gamma)$ satisfies $\|w\| \leq \delta$. Let $C:=\left\{w \in c_{0}(\mathbb{N}) ; \delta<\|w\| \leq 1\right\}$. We shall proof the following

Claim. There exists a sequence $\left(v_{n}\right)$ in $C \cap c_{00}(\Gamma)$ with the following properties:

(i) $\operatorname{supp} v_{n} \cap \operatorname{supp} v_{m}=\emptyset$ for all distinct $n, m \in \mathbb{N}$.

(ii) $\left\|T\left(v_{n}\right)\right\|>\varepsilon / 2$ for all $n \in \mathbb{N}$.

(iii) $\left\|T v_{n}-T v_{m}\right\|>\varepsilon / 2$, for all distinct $n, m \in \mathbb{N}$.

Proof of the Claim. Let us start by choosing any $v_{1} \in C$ such that $\left\|T v_{1}\right\|>\varepsilon / 2$. It exists because of (5.30). Obviously, we may assume that $v_{1} \in C \cap c_{00}(\Gamma)$. Suppose that $v_{1}, v_{2}, \ldots, v_{n}$ have already been defined for some $n \in \mathbb{N}$. If the construction could not be carried out, then

$$
\begin{aligned}
& \text { for all } w \in C \text { such that supp } w \cap \bigcup_{k=1}^{n} \operatorname{supp} v_{k}=\emptyset \\
& \quad \text { either there is } k \in\{1,2, \ldots, n\} \text { with }\left\|T w-T v_{k}\right\| \leq \varepsilon / 2, \\
& \quad \text { or }\|T w\| \leq \varepsilon / 2 .
\end{aligned}
$$

Accordingly, let $C=C_{2} \cap C_{3}$, where $C_{2}$ is the subset of $C$ where (5.31) holds and $C_{3}$ the subset of $C$ where (5.32) holds. Given $w_{2} \in C_{2}$, put $w_{2}=w_{2,1}+w_{2,2}$, where $w_{2,1}:=w_{2} \cdot \chi_{\bigcup_{k=1}^{n}} \operatorname{supp} v_{k}$. The set $\left\{w_{2,1} ; w_{2} \in C_{2}\right\}$ is precompact, since it is bounded and all its elements have the same finite support, hence it can be covered by a finite number of $\delta$-small sets and so $\left\{T w_{2,1} ; w \in C\right\}$ can be covered by a finite number of $\varepsilon / 2$-small sets. If the element $w_{2,2}$ is in $C$ we have, according to (5.31) and (5.32), that either $T w_{2,2}$ is in $\bigcup_{k=1}^{n}\left(T v_{k}+(\varepsilon / 2) B_{X}\right)$ or $\left\|T w_{2,2}\right\| \leq \varepsilon / 2$. On the other side, if $w_{3} \in C_{3}$ then $\left\|T w_{3}\right\| \leq \varepsilon / 2$. Putting things together it follows that $T C$ can be covered by a finite number of $\varepsilon$-small sets, a contradiction.

Hence, the inductive process for constructing the sequence $\left(v_{n}\right)$ can be carried out (at each step, it is clear that we can modify the vector $v_{n}$ defined there to belong to $\left.c_{00}(\Gamma)\right)$.

Since $\left\|T v_{n}-T v_{m}\right\|>\varepsilon / 2$, the set $\left\{T v_{n} ; n \in \mathbb{N}\right\}$ is not relatively compact. Therefore we can find two sequences $\left(n_{k}\right)$ and $\left(m_{k}\right)$ in $\mathbb{N}$ such that $\left(T v_{n_{k}}-T v_{m_{k}}\right)$ is a basic sequence [Woj91, Exercise II.B.4] (see Proposition 203 below).

Assume that the range of $\left(T v_{n_{k}}\right)$ is finite. Then $T\left(v_{m_{k}}\right)$ is a basic sequence. We can reverse the roles of $\left(v_{m_{k}}\right)$ and $\left(v_{n_{k}}\right)$. If both ranges are infinite, we may assume that the supports of $v_{n_{k}}-v_{m_{k}}$ are disjoint for different $k$ 's. In all cases we get a sequence $\left(w_{n}\right)$ in $C \cap c_{00}(\Gamma)$ with mutually disjoint supports and such that $\left(T w_{n}\right)$ is a basic sequence with $\left\|T w_{n}\right\|>\varepsilon / 2$ for all $n \in \mathbb{N}$. According to Propositions 183 and 184, the two sequences $\left(w_{n}\right)$ and $\left(T w_{n}\right)$ are basic sequences equivalent to the canonical basis of $c_{0}(\mathbb{N})$. This gives the conclusion.

\section{Proof of Theorem 188.}

Along the proof, we shall establish several lemmata and two propositions. Let $X$ be a Banach space and let $\Gamma$ be an infinite set. Assume that there exists a continuous 
linear operator $T: c_{0}(\Gamma) \rightarrow X$. For $\gamma \in \Gamma$, put $e_{\gamma}$ for the $\gamma$ th vector of the canonical basis of $c_{0}(\Gamma)$.

Lemma 194 The set $\left\{T e_{\gamma} ; \gamma \in \Gamma\right\}$ countably supports $X^{*}$.

Proof. Indeed, $\left\{\gamma \in \Gamma ;\left\langle T e_{\gamma}, x^{*}\right\rangle \neq 0\right\}=\left\{\gamma \in \Gamma ;\left\langle e_{\gamma}, T^{*} x^{*}\right\rangle \neq 0\right\}$ for every $x^{*} \in X^{*}$. This last set is countable, since $T^{*}$ maps $X^{*}$ into $\ell_{1}(\Gamma)$.

Lemma 195 Let $C \subset X$ be a closed convex subset of $X$ such that $0 \notin C$. Then $\left\{\gamma \in \Gamma ; T e_{\gamma} \in C\right\}$ is finite.

Proof. Let $(0<) \delta:=\operatorname{dist}(0, C)$. Assume that $\left\{T e_{\gamma} ; \gamma \in F\right\} \subset C$ for some finite set $F \subset \Gamma$. Since $C$ is convex, we have

$$
\frac{\sum_{\gamma \in F} T e_{\gamma}}{\# F} \in C .
$$

Then,

$$
\begin{array}{r}
\delta \leq\left\|\frac{\sum_{\gamma \in F} T e_{\gamma}}{\# F}\right\|=\frac{1}{\# F}\left\|T\left(\sum_{\gamma \in F} e_{\gamma}\right)\right\| \\
\leq \frac{1}{\# F}\|T\| .\left\|\sum_{\gamma \in F} e_{\gamma}\right\|_{\infty}=\frac{1}{\# F}\|T\| .
\end{array}
$$

It follows that $\# F \leq \frac{1}{\delta}\|T\|$, and hence

$$
\#\left\{\gamma \in \Gamma ; T e_{\gamma} \in C\right\} \leq \frac{1}{\delta}\|T\|(<+\infty) .
$$

This finishes the proof.

Remark 196 Observe that there is a uniformity in the number of elements $T e_{\gamma}$ in $C$ controlled by the distance from $C$ to 0 .

Let $T: c_{0}(\Gamma) \rightarrow X$ be a continuous linear operator with dense range. Let $\Gamma_{1}:=$ $\left\{\gamma \in \Gamma ; T e_{\gamma} \neq 0\right\}$ and put $\Gamma_{0}:=\Gamma \backslash \Gamma_{1}$. Since $c_{0}(\Gamma)$ is isometrically isomorphic to $c_{0}\left(\Gamma_{0}\right) \bigoplus c_{0}\left(\Gamma_{1}\right)$ equipped with the supremum norm (and both spaces $c_{0}\left(\Gamma_{0}\right)$ and $c_{0}\left(\Gamma_{1}\right)$ are 1-complemented in $c_{0}(\Gamma)$ ), by taking the restriction of $T$ to $c_{0}\left(\Gamma_{1}\right)$ (and calling $\Gamma_{1}$ again $\Gamma$ ) we may assume $T e_{\gamma} \neq 0$ for every $\gamma \in \Gamma$.

In the sequel we shall be dealing with the following situation.

$\left.{ }^{*}\right) X$ is a Banach space, $\Gamma$ an infinite set, $T: c_{0}(\Gamma) \rightarrow X$ a continuous linear operator with dense range, and $T e_{\gamma} \neq 0$ for every $\gamma \in \Gamma$.

We can now state and prove the following result. 
Proposition 197 Let $Y$ be a Banach space such that, for some $\varepsilon>0$, there exists an infinite set $\Gamma_{\varepsilon}$ and a continuous linear mapping $T: c_{0}\left(\Gamma_{\varepsilon}\right) \rightarrow Y$ with dense range, and such that $\left\|T e_{\gamma}\right\| \geq \varepsilon$ for every $\gamma \in \Gamma_{\varepsilon}$. Then $\operatorname{dens} Y=\# \Gamma_{\varepsilon}$.

Proof. Since $\left\{T e_{\gamma} ; \gamma \in \Gamma_{\varepsilon}\right\}$ is a linearly dense subset of $Y$, we certainly have dens $Y \leq \# \Gamma_{\varepsilon}$.

In order to prove the reverse inequality, let $S$ be a dense subset of $Y$. Put $S_{\varepsilon}:=$ $\{s \in S ;\|s\| \geq \varepsilon\}$. According to Lemma 195, the set $\left\{\gamma \in \Gamma_{\varepsilon} ; T e_{\gamma} \in B(s ; \varepsilon / 3)\right\}$ is finite for every $s \in S_{\varepsilon}$. So $\# \Gamma_{\varepsilon} \leq \# S_{\varepsilon} \leq \# S$. It follows that $\# \Gamma_{\varepsilon} \leq \operatorname{dens} Y$. This completes the proof.

Let $Y, \varepsilon, \Gamma_{\varepsilon}$, and $T$ be as in Proposition 197 (so dens $Y=\# \Gamma_{\varepsilon}$ ). Lemma 194 says, in particular, that $\left\{T e_{\gamma} ; \gamma \in \Gamma_{\varepsilon}\right\}$ countably supports $Y^{*}$.

Let $\mu$ be the first ordinal with cardinality $\# \Gamma_{\varepsilon}$. As it is well known (see, e.g., Proposition 30), there is a projectional resolution of the identity $\left(P_{\alpha}\right)_{\omega_{0} \leq \alpha \leq \mu}$ on $Y$ subordinated to $\left\{T e_{\gamma} ; \gamma \in \Gamma_{\varepsilon}\right\}$. For $\alpha \in\left[\omega_{0}, \mu\right)$ put $D_{\alpha}:=\left(P_{\alpha+1}-P_{\alpha}\right) \bar{Y}$.

Lemma 198 For every $\alpha \in\left[\omega_{0}, \mu\right)$, we have $D_{\alpha} \cap\left\{T e_{\gamma} ; \gamma \in \Gamma_{\varepsilon}\right\} \neq \emptyset$.

Proof. Without loss of generality, we may always assume that $D_{\alpha} \neq\{0\}$ for every $\alpha \in\left[\omega_{0}, \mu\right)$. Let $0 \neq y \in D_{\alpha}$. We can find a sequence $\left(z_{n}\right)$ in $\operatorname{span}\left\{T e_{\gamma} ; \gamma \in \Gamma_{\varepsilon}\right\}$ such that $z_{n} \stackrel{\|\cdot\|}{\longrightarrow} y$. Then, $\left(P_{\alpha+1}-P_{\alpha}\right) z_{n} \stackrel{\|\cdot\|}{\longrightarrow}\left(P_{\alpha+1}-P_{\alpha}\right) y(=y)$. So we can find $n \in \mathbb{N}$ such that $\left(P_{\alpha+1}-P_{\alpha}\right) z_{n} \neq 0$. Assume that $D_{\alpha} \cap\left\{T e_{\gamma} ; \gamma \in \Gamma_{\varepsilon}\right\}=\emptyset$. Then $\left(P_{\alpha+1}-P_{\alpha}\right) T e_{\gamma}=0$ for every $\gamma \in \Gamma_{\varepsilon}$. This is a contradiction.

Remark 199 In fact, the previous proof shows that $\left\{T e_{\gamma} ; \gamma \in \Gamma\right\} \cap D_{\alpha}$ is linearly dense in $D_{\alpha}$ for every $\alpha \in\left[\omega_{0}, \mu\right)$.

Choose, using Lemma 198, exactly one $T e_{\gamma} \in D_{\alpha}$ for each $\alpha \in\left[\omega_{0}, \mu\right)$. This defines a one-to-one mapping $\phi:\left[\omega_{0}, \mu\right) \rightarrow \Gamma_{\varepsilon}$.

Proposition $200\left\{T e_{\phi(\alpha)} ; \alpha \in\left[\omega_{0}, \mu\right)\right\}$ is a long Schauder basic sequence equivalent to the canonical basis of $c_{0}\left(\left[\omega_{0}, \mu\right)\right)$.

Proof. For $n \in \mathbb{N}$, take a finite set $\left\{\alpha_{1}<\alpha_{2}<\ldots<\alpha_{n}\right\} \subset\left[\omega_{0}, \mu\right)$ and a set $\left\{a_{1}, a_{2}, \ldots, a_{n}\right\}$ of scalars. Then, if $n>1$, for $k \in\{1,2, \ldots, n-1\}$ we have

$$
\left\|\sum_{i=1}^{k} a_{i} T e_{\phi\left(\alpha_{i}\right)}\right\|=\left\|P_{\alpha_{k+1}} \sum_{i=1}^{n} a_{i} T e_{\phi\left(\alpha_{i}\right)}\right\| \leq\left\|\sum_{i=1}^{n} a_{i} T e_{\phi\left(\alpha_{i}\right)}\right\| .
$$

In view of Proposition 179, $\left\{T e_{\phi(\alpha)} ; \alpha \in\left[\omega_{0}, \mu\right)\right\}$ is a long Schauder basic sequence. To prove that, indeed, it is equivalent to the canonical basis of $c_{0}\left(\left[\omega_{0}, \mu\right)\right)$, let $\left(b_{\alpha}\right)_{\omega_{0} \leq \alpha<\mu}$ be a long sequence of scalars. Assume first that $\sum_{\omega_{0} \leq \alpha<\mu} b_{\alpha} e_{\alpha}$ converges in $\left(c_{0}\left(\left[\omega_{0}, \mu\right)\right), \|\right.$. 
$\left.\|_{\infty}\right)$. A continuous linear mapping $J: c_{0}\left(\left[\omega_{0}, \mu\right)\right) \rightarrow c_{0}\left(\Gamma_{\varepsilon}\right)$ is defined by letting $J e_{\alpha}:=e_{\phi(\alpha)}$ for $\alpha \in\left[\omega_{0}, \mu\right)$. Certainly, $\|J\|=1$. Then we have

$$
\begin{gathered}
\left\|\sum_{\alpha \in\left[\omega_{0}, \mu\right)} b_{\alpha} T e_{\phi(\alpha)}\right\|=\left\|(T \circ J) \sum_{\alpha \in\left[\omega_{0}, \mu\right)} b_{\alpha} e_{\alpha}\right\| \\
\leq\|T\| \cdot\left\|\sum_{\alpha \in\left[\omega_{0}, \mu\right)} b_{\alpha} e_{\phi(\alpha)}\right\| .
\end{gathered}
$$

On the other hand, assume that $\left(b_{\alpha}\right)_{\alpha \in\left[\omega_{0}, \mu\right)}$ is a long sequence of scalars such that $\left\|\sum_{\alpha \in\left[\omega_{0}, \mu\right)} b_{\alpha} T e_{\phi(\alpha)}\right\|$ converges in $Y$. In particular, $\left\|b_{\alpha} T e_{\phi(\alpha)}\right\| \rightarrow 0$. Since for every $\alpha \in\left[\omega_{0}, \mu\right)$ we have $\left\|T e_{\phi(\alpha)}\right\| \geq \varepsilon$, it follows that $\left(b_{\alpha}\right)$ is a long null sequence, and so $\sum_{\alpha \in\left[\omega_{0}, \mu\right)} b_{\alpha} e_{\phi(\alpha)}$ converges, too. This proves the equivalence of both long basic sequences.

The proof of Theorem 188 follows directly from Proposition 200.

Remark 201 Note that in Theorem 188, if we take a Banach space $X$ with $\operatorname{cof}(\operatorname{dens} X)$ $>\# \omega_{0}$, the condition $\left\|T e_{\gamma}\right\| \geq \varepsilon$ for some $\varepsilon>0$ and for every $\gamma \in \Gamma$, can be omitted. In fact, put $\Gamma_{n}:=\left\{\gamma \in \Gamma ;\left\|T e_{\gamma}\right\|>\frac{1}{n}, n \in \mathbb{N}\right\}$. We get $\Gamma=\cup_{n \in \mathbb{N}} \Gamma_{n}$. Then there exists $n \in \mathbb{N}$ such that $\# \Gamma_{n} \geq$ dens $X$. Indeed, if not, for all $n \in \mathbb{N}$ we have $\# \Gamma_{n}<\operatorname{dens} X$. Therefore $\# \Gamma=\sup _{n \in \mathbb{N}} \# \Gamma_{n}<\operatorname{dens} X$, a contradiction. Observe that, if the condition $\operatorname{cof}(\operatorname{dens} X)>\# \omega_{0}$ is not satisfied, we can find the following counterexample.

Consider mutually disjoint sets $\Gamma_{1}, \Gamma_{2}, \ldots$ such that $\# \Gamma_{n}=\# \omega_{n}$ for every $n \in \mathbb{N}$. Put $\Gamma=\bigcup_{n \in \mathbb{N}} \Gamma_{n}$. Then $\# \Gamma=\sup \left\{\omega_{n} ; n \in \mathbb{N}\right\}=\omega_{\omega_{0}}$. Define the Banach space $X=\left(\sum_{n=1}^{\infty} c_{0}\left(\Gamma_{n}\right)\right)_{\ell_{2}}$ and define the operator $T: c_{0}(\Gamma) \rightarrow X$ by $T a(n)=\frac{1}{n} a_{\mid \Gamma_{n}}$, for all $n \in \mathbb{N}$ and $a \in c_{0}(\Gamma)$. We have

$$
\|T a\|^{2}=\sum_{n=1}^{\infty}\|T a(n)\|^{2}=\sum_{n=1}^{\infty} \frac{1}{n^{2}}\left\|\left.a\right|_{\Gamma_{n}}\right\|_{\infty}^{2} \leq\|a\|_{\infty}^{2} \sum_{n=1}^{\infty} \frac{1}{n^{2}},
$$

so $T$ is linear, bounded and has dense range.

Assume now that there exists $\Gamma^{\prime} \subset \Gamma$ with $\# \Gamma^{\prime}=\# \Gamma$ and such that $\left.T\right|_{\Gamma^{\prime}}$ is an isomorphism. Find $c>0$ such that $\left\|T\left(\left.a\right|_{\Gamma^{\prime}}\right)\right\| \geq c\left\|\left.a\right|_{\Gamma^{\prime}}\right\|_{\infty}$. Since $\# \Gamma^{\prime}=\omega_{\omega_{0}}$, there exist an infinite set $K \subset \mathbb{N}$ such that, for every $k \in K$, we can find $\gamma_{k} \in \Gamma^{\prime} \cap \Gamma_{k}$. For $k>\frac{1}{c}$, we have

$$
c=c\left\|e_{\gamma_{n}}\right\| \leq\left\|T e_{\gamma_{k}}\right\|=\left\|\frac{1}{k} e_{\gamma_{k}}\right\|=\frac{1}{k}<c,
$$

and this is a contradiction.

Remark 202 We were not able to find a proof of Theorem 191 following the same pattern as in the proof of Theorem 188. 
Proof of Corollary 192. We use the argument from Remark 201. Consider $\Gamma_{n}:=$ $\left\{\gamma \in \omega_{1} ;\left\|T e_{\gamma}\right\| \geq 1 / n\right\}, n \in \mathbb{N}$, and let $T: c_{0}\left(\omega_{1}\right) \rightarrow X$ be the existing linear bounded operator. Since the cofinality of $\omega_{1}$ is $\aleph_{1}$, at least one of $\Gamma_{n}$ must have cardinality $\aleph_{1}$. It follows from Proposition 197 that the density of $T\left(c_{0}\left(\Gamma_{n}\right)\right)$ is $\omega_{1}$, and we can apply Theorem 188.

Proof of Corollary 193. (i) By Theorem 156, there exists a bounded linear mapping $T: c_{0}(\Gamma) \rightarrow X$ with dense image. We can apply now Corollary 192. (ii) In this case, use Theorem 158 instead of Theorem 156 and repeat the argument above. 



\section{Chapter 6}

\section{Appendix}

\subsection{Appendix A}

The aim of this section is twofold. First, to prove the following result, which appears as a proposed exercise — without hints - in [Woj91, page 44] and that was used in the proof of Theorem 186.

Proposition 203 Let $X$ be a Banach space. Let $\left(x_{n}\right)$ be a bounded and not relatively compact sequence in $X$. Then, there exists two subsequences $\left(y_{n}\right)$ and $\left(z_{n}\right)$ of $\left(x_{n}\right)$ such that the sequence $\left(d_{n}:=y_{n}-z_{n}\right)$ is basic.

Proof. Since $\left\{x_{n} ; n \in \mathbb{N}\right\}$ is not precompact, there exists $\delta>0$ and a subsequence of $\left(x_{n}\right)$ (denoted again $\left(x_{n}\right)$ ) such that $\left\|x_{n}-x_{m}\right\| \geq \delta$ for every $n, m \in \mathbb{N}$ such that $n \neq m$. Moreover, the set $\left\{x_{n} ; n \in \mathbb{N}\right\}$ is infinite, so there exists a $w^{*}$-accumulation point $x^{* *} \in X^{* *}$ of $\left(x_{n}\right)$.

We follow an idea that goes back to Mazur. Fix $\varepsilon_{0} \in(0,1)$, and find $\varepsilon_{n}>0, n \in \mathbb{N}$, such that $\prod_{i=1}^{\infty}\left(1-\varepsilon_{n}\right) \geq\left(1-\varepsilon_{0}\right)$. To define $\left(y_{n}\right)$ and $\left(z_{n}\right)$ proceed recursively. First, put $y_{1}:=x_{1}$ and $z_{1}:=x_{2}$. Assume that $y_{1}, \ldots, y_{n}$ and $z_{1}, \ldots, z_{n}$ have been already defined. Let $F:=\operatorname{span}\left\{y_{i}, z_{i} ; i=1,2, \ldots, n\right\}$, and let $\left\{r_{1}, \ldots, r_{m}\right\}$ be an $\varepsilon_{n} / 2$-net in $S_{F}$. Find $\left\{f_{1}, \ldots, f_{m}\right\} \subset S_{X^{*}}$ such that $\left\langle r_{k}, f_{k}\right\rangle=1$ for $k=1,2, \ldots, m$. Since $\left\{x_{n} ; n \in \mathbb{N}\right\}$ has a $w^{*}$-accumulation point in $X^{* *}$, we can find $p, q \in \mathbb{N}$ big enough to have $\left|\left\langle x_{p}-x_{q}, f_{i}\right\rangle\right|<(\delta / 4) \varepsilon_{n}$, for $i=1,2, \ldots, m$. Put $y_{n+1}:=x_{p}, z_{n+1}=x_{q}$. If $d_{n+1}:=y_{n+1}-z_{n+1}$, we get $\left\|d_{n+1}\right\| \geq \delta$ and $\left|\left\langle d_{n+1}, f_{i}\right\rangle\right|<(\delta / 4) \varepsilon_{n}$, for $i=1,2, \ldots, m$. Take $\left\{a_{i} ; i=1,2, \ldots, n+1\right\} \subset \mathbb{R}$ such that $\left\|\sum_{i=1}^{n} a_{i} d_{i}\right\|=1$. Then there exists $k \in\{1,2, \ldots, m\}$ such that $\left\|\sum_{i=1}^{n} a_{i} d_{i}-r_{k}\right\|<\varepsilon_{n} / 2$. Assume first $\left|a_{n+1}\right| \geq 2 / \delta$. 
Then

$$
\begin{aligned}
& \left\|\sum_{i=1}^{n+1} a_{i} d_{i}\right\| \geq\left|a_{n+1}\right|\left\|d_{n+1}\right\|-\left\|\sum_{i=1}^{n} a_{i} d_{i}\right\| \\
& \quad=\left|a_{n+1}\right|\left\|d_{n+1}\right\|-1 \geq\left|a_{n+1}\right| \delta-1 \geq 2-1=\left\|\sum_{i=1}^{n} a_{i} d_{i}\right\| .
\end{aligned}
$$

If, on the other hand, $\left|a_{n+1}\right|<2 / \delta$, we have

$$
\begin{aligned}
& \left\|\sum_{i=1}^{n+1} a_{i} d_{i}\right\| \geq\left|\left\langle\sum_{i=1}^{n+1} a_{i} d_{i}, f_{k}\right\rangle\right| \\
& =\left|\left\langle r_{k}, f_{k}\right\rangle+\left\langle\sum_{i=1}^{n} a_{i} d_{i}-r_{k}, f_{k}\right\rangle+\left\langle a_{n+1} d_{n+1}, f_{k}\right\rangle\right| \\
& \quad \geq 1-\frac{\varepsilon_{n}}{2}-\frac{2}{\delta} \frac{\delta}{4} \varepsilon_{n}=\left(1-\varepsilon_{n}\right)=\left(1-\varepsilon_{n}\right)\left\|\sum_{i=1}^{n} a_{i} d_{i}\right\| .
\end{aligned}
$$

By homogeneity, we get $\left\|\sum_{i=1}^{n+1} a_{i} d_{i}\right\| \geq\left(1-\varepsilon_{n}\right)\left\|\sum_{i=1}^{n} a_{i} d_{i}\right\|$ for every family $\left\{a_{i} ; i=\right.$ $1,2, \ldots, n+1\} \subset \mathbb{R}$. This proves that, for every finite number of scalars $\left\{a_{i} ; i=\right.$ $1,2, \ldots, q\}$ and for $1 \leq p \leq q$ we have $\left\|\sum_{i=1}^{q} a_{i} d_{i}\right\| \geq\left(1-\varepsilon_{0}\right)\left\|\sum_{i=1}^{p} a_{i} d_{i}\right\|$, and this finishes the proof.

The second part of this appendix is an attempt to provide a partial result about fixing $\ell_{\infty}(\Gamma)$. Of course, we shall not use Theorem 191, since the aim was to try to provide a proof of this result by just using our approach (see the list of open problems at Section 6.3. It is clear, by a simple argument, that the non-angelicity of $\left(B_{X^{*}}, w^{*}\right)$ is a consequence of the existence of a copy of $\ell_{\infty}(\Gamma)$ in $X$.

Proposition 204 Let $T: \ell_{\infty}(\Gamma) \rightarrow X$ be a bounded linear operator such that, for some $\delta>0,\left\|T e_{\gamma}\right\| \geq \delta$ for all $\gamma \in \Gamma$. Then, $\left(B_{X^{*}}, w^{*}\right)$ is not angelic.

Proof. Assume that $T: \ell_{\infty}(\Gamma) \rightarrow X$ is a bounded linear operator such that, for some $\delta>0,\left\|T e_{\gamma}\right\| \geq \delta$ for all $\gamma \in \Gamma$. We proved in Theorem 188 using the Remark 201 that there exists a subset $\Gamma^{\prime} \subset \Gamma$ such that $\# \Gamma^{\prime}=\# \Gamma$, and $\hat{T}:=T \uparrow_{c_{0}\left(\Gamma^{\prime}\right)}$ is an isomorphism into. Denote again by $T$ the restriction of $T$ to $\ell_{\infty}\left(\Gamma^{\prime}\right)$. Obviously, $\ell^{*}\left(\Gamma^{\prime}\right)=\ell_{1}\left(\Gamma^{\prime}\right) \oplus c_{0}^{\perp}\left(\Gamma^{\prime}\right)$. Let $R_{1}: \ell^{*}\left(\Gamma^{\prime}\right) \rightarrow \ell_{1}\left(\Gamma^{\prime}\right)$ be the associated projection on the first coordinate. It is clear that $R_{1} \circ T^{*}=\hat{T}^{*}$. Let $\left(x_{n}^{*}\right)$ be a $w^{*}$-null sequence in $X^{*}$. Then, $T^{*} x_{n}^{*} \stackrel{w^{*}}{\longrightarrow} 0$, hence, by the Grothendieck property of $\ell_{\infty}(\Gamma), T^{*} x_{n}^{*} \stackrel{w}{\longrightarrow} 0$. It follows that $R_{1} T^{*} x_{n}^{*} \stackrel{w}{\longrightarrow} 0$, so, by the Schur property of $\ell_{1}\left(\Gamma^{\prime}\right), R_{1} T^{*} x_{n}^{*} \stackrel{\|\cdot\|}{\longrightarrow} 0$, i.e., $\hat{T}^{*} x_{n}^{*} \stackrel{\|\cdot\|}{\longrightarrow} 0$. Assume that $\left(B_{X^{*}}, w^{*}\right)$ is angelic. Then $\hat{T}^{* *}$ maps $\ell_{\infty}\left(\Gamma^{\prime}\right)$ into $X$. Indeed, let $l \in \ell_{\infty}\left(\Gamma^{\prime}\right)$. Given a $w^{*}$-null sequence $\left(x_{n}^{*}\right)$ in $B_{X^{*}}$,

$$
\left\langle\hat{T}^{* *} l, x_{n}^{*}\right\rangle=\left\langle l, \hat{T}^{*} x_{n}^{*}\right\rangle \rightarrow 0 .
$$


This implies that $\hat{T}^{* *} l$ is, by the Banach-Dieudonné Theorem, $w^{*}$-continuous, and so $\hat{T}^{* *}$ maps $\ell_{\infty}\left(\Gamma^{\prime}\right)$ into $X$. Obviously, $\hat{T}^{* *}$ is again an isomorphism into. This proves that $X$ contains an isomorphic copy of $\ell_{\infty}\left(\Gamma^{\prime}\right)$, and this is a contradiction with the angelicity of $\left(B_{X^{*}}, w^{*}\right)$.

\subsection{Appendix B}

This section has the purpose to complement and justify what has been said in Remark 162.

Definition 205 We say that a Banach space $X$ is strongly generated by a Banach space $Z$ if there is a bounded linear operator $T$ from $Z$ into $X$ such that, for every weakly compact set $W \subset X$ and every $\varepsilon>0$, there exists $m \in \mathbb{N}$ such that $W \subset$ $m T\left(B_{Z}\right)+\varepsilon B_{X}$. In this case we say, too, that $Z$ strongly generates $X$.

Remark 206 Definition 205 is motivated by the concept of a strongly weakly compactly generated Banach space ( $\beta \mathrm{WCG}$, in short), introduced by Schluchtermann and Wheeler [SW88]: A Banach space $X$ is $\beta$ WCG if there exists a weakly compact subset $K \subset X$ such that, for every weakly compact subset $W \subset X$, we can find $n \in \mathbb{N}$ such that $W \subset n K+\varepsilon B_{X}$ (we say, in this case, that $K$ strongly generates $X$, or that $X$ is strongly generated by $K$, hoping that it does not cause any misunderstanding with Definition 205). Obviously, if $X$ is strongly generated by a reflexive space $Z$ then it is $\beta$ WCG. The converse, a straightforward consequence of the factorization theorem of Davis, Figiel, Johnson and Pełczyński [DFJP74], holds. Precisely, if $K \subset X$ is a weakly compact subset strongly generating $X$, then there exists a reflexive Banach space $Z$ and a bounded linear mapping $T: Z \rightarrow X$ such that $K \subset T\left(B_{Z}\right)$, and so $Z$ strongly generates $X$.

Note, too, that if $X$ is strongly generated by a Banach space $Z$ via a bounded linear mapping $T$, then $X$ is strongly generated by the quotient $Z / \operatorname{ker} T$ and now the induced strongly generating mapping $\hat{T}: Z / \operatorname{ker} T \rightarrow X$ is one-to-one.

Proposition 207 If $\mu$ is a finite measure defined on a $\sigma$-algebra $\Sigma$ of subsets of a certain set $\Omega$, then the space $L_{1}(\Omega, \Sigma, \mu)$ is strongly generated by a Hilbert space.

Proof. We shall use [JL01, page 17]. Assume without loss of generality that $\mu$ is a probability measure. By using the identity operators, we have $B_{L_{\infty}(\mu)} \subset B_{L_{2}(\mu)} \subset$ $B_{L_{1}(\mu)}$. Let $K$ be a weakly compact set in the unit ball of $L_{1}(\mu)$. Then $K$ is uniformly integrable in $L_{1}(\mu)$ ([DS67, page 292]), i.e. for every $\varepsilon>0$ there is $\delta>0$ such that for every $x \in K$, we have $\int_{M}|x| d \mu<\varepsilon$ whenever $M \in \Sigma$ and $\mu(M)<\delta$.

For $k \in \mathbb{N}$ and for $x \in K$, put $M_{k}(x):=\{t \in \Omega ;|x(t)| \geq k\}$, and write $x=$ $x_{1}+x_{2}$, where $x_{1}:=x \cdot \chi\left(\Omega \backslash M_{k}(x)\right)$ and $x_{2}:=x \cdot \chi\left(M_{k}(x)\right.$ ) (where $\chi(S)$ denotes the characteristic function of a set $S \subset \Omega)$. Let $a_{k}(K):=\sup \left\{\left\|x_{2}\right\|_{1} ; x \in K\right\}$. Then

$$
K \subset k B_{L_{\infty}(\mu)}+a_{k}(K) B_{L_{1}(\mu)} \subset k B_{L_{2}(\mu)}+a_{k}(K) B_{L_{1}(\mu)} .
$$


We have $k \mu\left(M_{k}(x)\right) \leq\left\|x_{2}\right\|_{1} \leq 1$, hence $\mu\left(M_{k}(x)\right) \leq 1 / k$ for all $x \in K$. From the uniform integrability of $K$, we get that $a_{k}(K) \rightarrow 0$ when $k \rightarrow \infty$. This finishes the proof.

\subsection{Appendix C. Some problems and further sug- gested developments}

The following is a list of some of problems encountered along the Memoir that we were not able to solve. We plan to continue our research hoping that new techniques/ideas will help to solve them.

1. We do not know whether the example provided by Leiderman (see Example 12 ) is a Gruenhage compactum. J. Orihuela suggested the following related question: to know whether the same example is a descriptive compact space (if this would be the case, then $C(K)^{*}$ will have an equivalent $w^{*}$-LUR norm), or whether it belongs to the classes introduced in [OR04].

2. We do not know a simpler proof (simpler than the one that can be found in [Di75], due to J. Rainwater) to ensure that the norm introduced in Chapter 3 is, in fact, LUR. To be precise, we know that this norm is already LUR. To prove this, we elaborated a proof that, after adjustments, turned out to be quite similar to the Rainwater's proof, so we preferred not to include it here, since did not add any new idea.

3. We do not know whether the concept introduced in [BGV99] can be used instead of the usual moduli of rotundity and smoothness to conclude that, under this behavior, our sets are $\|\cdot\|$-asymptotically $p$-flat, using an approach close to the one in Proposition 129.

4. As it was mentioned in Remark 140, Proposition 138 and Corollary 139 give only relevant information for $L_{p}$-spaces in the case $p \in[1,2)$. The natural conjecture is that for spaces $L_{p}$ a result similar to Proposition 136 holds. We were unable to prove or disprove this conjecture.

5. We do not know how to extend the theory developed in Chapter 5 to higher ordinals. To say the truth, we are able to prove that every WCG Banach space $(X,\|\cdot\|)$ of density $\aleph$, where the first ordinal $\mu$ with cardinality $\aleph$ is uncountable, regular and limit of a sequence of regular ordinals, and such that its closed unit ball is $\|\cdot\|$-asymptotically $p$-flat with constant $C=1$, is generated by $\ell_{p}(\mu)$. This result seems too special to be presented here. Our conjecture is that a result similar to Theorem 156 holds with very mild cardinality restrictions.

All's Well That Ends Well 


\section{Bibliography}

[Ab72] A. Abramovich, Weakly compact sets in topological K-spaces, Theor. Funktsii Funktsional. Anal. I Prilozhen 15 (1972), 27-35.

[AmLi68] D. Amir and J. Lindenstrauss, The structure of weakly compact sets in Banach spaces, Ann. of Math. 88 (1968), 35-44.

[ACGJM] S. A. Argyros, J. F. Castillo, A. S. Granero, M. Jiménez, and J. P. Moreno, Complementation and embeddings of $c_{0}(I)$ in Banach spaces, Proc. London Math. Soc. 85 (2002), 742-768.

[ArFa] S. Argyros, V. Farmaki, On the structure of weakly compact subsets of Hilbert spaces and applications to the geometry of Banach spaces, Trans. Amer. Math. Soc. 289(1985), 409-427.

[ArgMe] S. Argyros, S. Mercourakis, Examples concerning heredity problems of WCG Banach spaces, Proc. Amer. Math. Soc. 133 (2005), 773-785.

[BGV99] M. O. Bartlett, J. R. Giles and J. D. Vanderwerff, Directional moduli of rotundity and smoothness, Comment. Math. Univ. Carolinae 40,1 (1999), $39-51$.

[Beau82] B. Beauzamy, Introduction to Banach Spaces and Their Geometry. Mathematics Studies. North Holland 68, 1982.

[BRW77] Y. Benyamini, M.E. Rudin and M. Wage, Continuous images of weakly compact subsets of Banach spaces, Pacific J. Math. 70 (1977), 309û-324.

[BP75] C. Bessaga and A. Pełczyński, Selected topics in infinite-dimensional topology, Polish Sci. Publ., Warszawa, 1975.

[CNO03] B. Cascales, I. Namioka, and J. Orihuela, The Lindelöf property in Banach spaces. Studia Math. 154 (2003), no.2, 165-192.

[Clark36] J.A. Clarkson, Uniformly convex spaces, Trans. Amer. Math. Soc. 40 (1936), 396-414. 
[DFJP74] W.J. Davis, T. Figiel, W.B. Johnson and A. Pełczyński, Factoring weakly compact operators, J. Funct. Anal. 17 (1974), 311-327.

[Day41] M. M. Day, Some more uniformly convex spaces. Bull. Amer. Math. Soc. 47 (1941), 504-507.

[Day55] M. M. Day, Strict convexity and smoothness, Trans. AMS78, (1955), 516528.

[DGZ] R. Deville, G. Godefroy and V. Zizler, Smoothness and renormings in Banach spaces. Pitman Monographs and Surveys in Pure and Applied Mathematicas 64. Longman Scientific \& Technical, Harlow, 1993.

[Di75] J. Diestel, Geometry of Banach spaces. Selected topics, Lecture Notes in Math. 485, Springer Verlag 1975.

[Di84] J. Diestel, Sequences and series in Banach spaces. GTM 92, Springer Verlag, 1984.

[DiUh77] J. Diestel and J. Uhl, Vector measures, Math. surveys 15, Amer. Math. Soc., 1977.

[DS67] N. Dunford and J.T. Schwartz, Linear operators, Part I: General theory, Interscience Publishers, Inc. New York, 1967.

[Enf73] P. Enflo, A counterexample to the approximation property in Banach spaces, Acta Math. 130 (1973), 309-317.

[Fab87] M. Fabian, On projectional resolution of identity on the duals of certain Banach spaces, Bull. Australian Math. Soc. 35(1987), 363-372.

[Fab87-b] M. Fabian, Each weakly countably determined Asplund space admits a Fréchet differentiable norm, Bull. Australian Math. Soc. 36(1987), 367374 .

[Fab97] M. Fabian, Gateaux Differentiability of Convex Functions and Topology —Weak Asplund Spaces, John Wiley \& Sons, New York, 1997.

[FG88] M. Fabian and G. Godefroy, The dual of every Asplund space admits a projectional resolution of identity, Studia Math. 91 (1988), 141-û151.

[FGHZ03] M. Fabian, G. Godefroy, P. Hájek, and V. Zizler, Hilbert-generated spaces, J. Functional Analysis 200 (2003), 301-323.

[FGMZ04] M. Fabian, G. Godefroy, V. Montesinos and V. Zizler, Inner characterizations of weakly compactly generated Banach spaces and their relatives, J. Math. Anal. and Appl., 297 (2004), 419-455. 
[FGZ01] M. Fabian, G. Godefroy, and V. Zizler, The structure of uniformly Gâteaux smooth Banach spaces, Israel J. Math. 124 (2001), 243-252.

[FGM] M. Fabian, A. González, and V. Montesinos, A note on biorthogonal systems in weakly compactly generated Banach spaces. To appear.

[FGZ] M. Fabian, A. González, and V. Zizler, Flat sets, $\ell_{p}$-generating and fixing $c_{0}$ in nonseparable setting. To appear.

[FHHMPZ01] M. Fabian, P. Habala, P. Hájek, V. Montesinos, J. Pelant, and V. Zizler, Functional Analysis and Infinite Dimensional Geometry, Canad. Math. Soc. Books in Mathematics 8, Springer-Verlag, New York, 2001.

[FHMZ] M. Fabian, P. Hájek, V. Montesinos and V. Zizler, A quantitative version of Krein's theorem, Revista Matemática Iberoamericana 21 No. 1, (2005), $237-248$.

[FMZ05] M. Fabian, V. Montesinos and V. Zizler, Biorthogonal systems in Weakly Lindelöf Spaces, Canadian Mathematical Bull. 48, 1 (2005), 69-79.

[FMZ04] M. Fabian, V. Montesinos and V. Zizler, A characterization of subspaces of weakly compactly generated Banach spaces, J. London Math. Soc. (2) 69 (2004), 457-464.

[FMZ07] M. Fabian, V. Montesinos, and V. Zizler, Weak compactness and sigmaAsplund generated Banach spaces, Studia Math. 181 (2007), 125-152.

[FMZ] M. Fabian, V. Montesinos, and V. Zizler, Sigma-finite dual dentability indices. To appear in J. Math. Anal. and Appl.

[FMZ02] M. Fabian. V. Montesinos, V. Zizler, Weakly compact sets and smooth norms in Banach spaces, Bull. Australian Math. Soc. 65 (2002), 223-230.

[Far87] V. Farmaki, The structure of Eberlein, uniformly Eberlein and Talagrand compact spaces in $\Sigma\left(\mathbb{R}^{\Gamma}\right)$. Fundamenta Math. 128, 15-28.

[Fig76] T. Figiel, On the moduli of convexity and smoothness, Studia Math. 66 (1976), 121-155.

[Flo80] K. Floret, Weakly compact sets, Lecture Notes in Math. 801, SpringerVerlag (1980).

[Frem84] D.H. Fremlin, Consequences of Martin's axioms, Cambridge University Press, 1984.

[GuKa62] V.I. Gurarii and M.I. Kadec, Minimal systems and quasicomplements in Banach spaces, Soviet Math. Dokl. 3 (1962), 966-968. 
[G01] G. Godefroy Renormings of Banach spaces, in Handbook of the geometry of Banach spaces, Ed. W. Johnson and J. Lindestrauss, Vol. I, p. 781-835, Elsevier, 2001.

[GKL00] G. Godefroy, N. Kalton, and G. Lancien, Subspaces of $c_{0}(\mathbb{N})$ an Lipschitz iomorphisms, Gafa, Geom. Funct. Anal. 10 (2000), 798-820.

[GM] A. González and V. Montesinos, A note on weakly Lindelöf determined Banach spaces. To appear in Czech. Math. Journal.

[Gr52] A. Grothendieck, Critères de compacité dans les espaces fonctionnels généraux. Amer. J. Math. 74 (1952), 168-186.

[Gul79] S. P. Gul'ko, On the structure of spaces of continuous functions and their complete paracompactness, Russian Math. Surveys 34(1979), 36-44; Uspekchi Mat. Nauk 34 (1979), 33-40.

[HLM07] P. Hájek, G. Lancien, and V. Montesinos, Universality of Asplund spaces, Proc. Amer. Math. Soc. 135 (2007), 2031-2035.

[HM] P. Hájek and V. Montesinos, Bounded biorthogonal systems in Banach spaces. To appear.

[HMVZ07] P. Hájek, V. Montesinos, J. Vanderwerff, V. Zizler, Biorthogonal Systems in Banach spaces, Canad. Math. Soc. Books in Mathematics, SpringerVerlag, CMS, November, 2007.

[Haj61] A. Hajnal, Proof of a conjecture of S. Ruziewicz, Fund. Math. 50 (1961), $123-128$.

[JOPV93] J. E. Jayne, J. Orihuela, A. Pallarés, and G. Vera, $\sigma$-Fragmentability of multivalued mappings and selections theorems, J. Funct. Anal. 117, (1993), 243-273.

[JaRo84] J. E. Jayne and C. A. Rogers, Borel selectors for upper semi-continuous multivalued functions, J. Funct. Anal. 56 (1984), 279-299.

[JZ74] K. John, V. Zizler, Duals of Banach space which admit nontrivial smooth functions, Bull. Australian Math. Soc. 11(1974), 161-166.

[JZ74-b] K. John, V. Zizler, Smoothness and its equivalents in weakly compactly generated Banach spaces, J. Funct. Anal. 15 (1974), 1-11.

[JZ77] K. John and V. Zizler, Some notes on Markushevich basis in weakly compactly generated Banach spaces, Compositio Math. 35 (1977), 113-123.

[JoLi01h] W.B. Johnson and J. Lindenstrauss, Editors, Handbook of the Geometry of Banach spaces, Elsevier, 2001. 
[JL01] W.B. Johnson and J. Lindenstrauss, Basic concepts in the geometry of Banach spaces, in Handbook of the Geometry of Banach Spaces, W.B. Johnson and J. Lindenstrauss eds., Elsevier, Vol. 1, p.1-84.2001.

[Kam50] E. Kamke, Theory of Sets, Dover Pub. Inc., 1950.

[Ko69] G. Köthe, Topological Vector Spaces I. Springer-Verlag, 1969.

[La95] G. Lancien, On uniformly convex and uniformly Kadec-Klee renormings, Serdica Math. J. 21 (1995), 1-18.

[LS84] A.G. Leiderman and G.A. Sokolov, Adequate families of sets and Corson compacta, Comment. Math. Univ. Carolinae 25 (1984), 233-̂̂u246.

[L1] J. Lindenstrauss, On reflexive spaces having the metric approximation property, Israel J. Math. 3(1965), 199-204.

[L2] J. Lindenstrauss, On nonseparable reflexive Banach spaces, Bull. Amer. Math. Soc. 72 (1966), 967-970.

[LT77] J. Lindenstrauss and L. Tzafriri , Classical Banach Spaces I, Sequence Spaces, Springer- Verlag, 1977.

[LT79] J. Lindenstrauss and L. Tzafriri , Classical Banach Spaces II, Function Spaces, Springer- Verlag, 1979.

[M43] A. I. Markushevich, On a basis in the wide sense for linear spaces, Dokl. Akad. Nauk. 41 (1943), 241-244.

[M71] V. D. Milman, Geometric theory of Banach spaces II. Geometry of the unit ball (Russian), Uspehi Mat. Nauk. 26 (1971), 6 (162), 73-149. English translation: Russian Math. Surveys 26 (1971), 6, 79-163.

[Na] I. Namioka, Eberlein and Radon-Nikodým compact spaces. Unpublished manuscript.

[NaPh75] I. Namioka and R. R. Phelps, Banach spaces which are Asplund spaces, Duke Math. J. 42 (1975), 735-750.

[NaWh86] I. Namioka and R. F. Wheeler, Gul'ko's proof of the Amir-Lindenstrauss theorem. Contemporary Mathematics, 52 (1986), 113-120.

[Nor] Nördlander, G., The modulus of convexity in normed linear spaces. Arkiv. Mat. 4 (1960), 15-17.

[OR04] L. Oncina, M. Raja, Descriptive compact spaces and renorming. Studia Math. 165 (2004), no.1, 39-52. 
[092] J. Orihuela, On weakly Lindelöf Banach spaces, Progress in Funct. Anal. K.D. Bierstedt, Editors J. Bonet, J. Horváth and M. Maestre, Elsevier Science Publ. B. V., 1992.

[OV89] J. Orihuela, M. Valdivia, Projective generators and resolutions of identity in Banach spaces, Rev. Mat. Univ. Complutense, Madrid 2, Suppl. Issue (1989), 179-199.

[Pis74] G. Pisier, Martingales à valeurs dans les espaces uniformément convexes. C. R. Acad. Sc. Paris, série A, 279 (1974), 647-649.

[Pt59] Pták, V., Biorthogonal systems and reflexivity of Banach spaces. Czechoslovak Math. J 9 (84) (1959), 319-326.

[Rain69] J. Rainwater, Local uniform convexity of Day'norm on $c_{0}(\Gamma)$, Proc. A. M. S. 22 (1969), 335-339.

[R70] H.P. Rosenthal, On relatively disjoint families of measures, with some applications to Banach space theory, Studia Math. 37 (1970), 13-30.

[Ros74] H. P. Rosenthal, The heredity problem for weakly compactly generated Banach spaces, Compositio Math. 28 (1974), 83-111.

[Rych04] J. Rychtář, On biorthogonal systems and Mazur's intersection property, Bull. Austr. Math. Soc. 69 (2004), 107-111.

[SW88] G. Schlüchtermann and R.F. Wheeler, On strongly WCG Banach spaces, Math. Z. 199 (1988), 387-398.

[T70] D. G. Tacon, The conjugate of a smooth Banach space, Bull. Australian Math. Soc. 2(1970), 415-425.

[Tal79] M. Talagrand, Espaces de Banach faiblement $\mathcal{K}$-analytiques, Ann. Math. 110 (1979), 407-438.

[Ter83] P. Terenzi, Extension of uniformly minimal M-basic sequences in Banach spaces. J. London Math. Soc. (2) 27 (1983), 500-506.

[Ter87] P. Terenzi, On the theory of fundamental bounded biorthogonal systems in Banach spaces. Trans. Amer. Math. Soc. (299) 2 (1987), 497-511.

[Val88] M. Valdivia, Resolution of the identity in certain Banach spaces, Collect. Math. 39(1988), 127-140.

[Val90] M. Valdivia, Projective resolution of identity in $C(K)$ spaces. Arch. Math. (Basel) 54 (1990), no.5, 493-498.

[Val91] M. Valdivia, Simultaneous resolutions of the identity operator in normed spaces, Collect. Math. 42 (1991), 265-284. 
[Val96] M. Valdivia, Biorthogonal systems in certain Banach spaces. Revista Mat. Univ. Complut. Madrid, 9 (1996), 191,220.

[Vand95] J. Vanderwerff, Extensions of Markuševič bases, Math. Z. 219 (1995), 21-30.

[VWZ94] J. Vanderwerf, J. H. M. Whitfield, and V. Zizler, Markushevich bases and Corson compacta in duality, Canad. J. Math. 46 (1994), 200-211.

[Woj91] P. Wojtaszczyk, Banach Spaces for Analysts, Cambridge Studies in Advanced Mathematics 25, Cambridge University Press, Cambridge, 1991.

[Xu91] H.K. Xu, Inequalities in Banach spaces with applications, Nonlinear Anal. TMA 16 (12) (1991), 1127-1138

[Zi68] M. Zippin, A remark on bases and reflexivity in Banach spaces, Israel J. Math. 6 (1968), 74-79.

[Z03] V. Zizler, Nonseparable Banach spaces, in Handbook of the Geometry of Banach spaces, Vol. 2, Edited by W.B. Johnson and J. Lindenstrauss, Elsevier 2003, 1743-1816. 


\section{Index}

Abramovich, A., 111

Amir, D., 6, 7, 32, 44, 111, 115

Amir-Lindenstrauss property, 6, 41, 42, 44

Argyros, S. A., 6, 42, 46, 47, 100, 111

Bartlett, M. O., 110, 111

basis

long Schauder, 94-97

Markushevich, 2, 5, 6, 21, 22, 24, 25, $27,28,32,35,37,38,42,83,85$

Beauzamy, B., 43, 111

Benyamini, Y., 111

Bessaga, C., 94, 95, 111

biorthogonal system, 2, 5, 6, 21, 22, 39, $41,43,48,113$

Cascales, B., vii, 28, 33, 36, 111

Castillo, J. F., 100, 111

Clarkson, J. A., 73, 111

compact

Corson, 2, 3, 5, 35-38, 85, 115

Eberlein, 2-4, 13, 18, 70, 113, 115

Gul'ko, 3, 4, 11-13, 15, 16, 18, 19, 49

Davis, W. J., 109, 112

Day, M. M., 6, 73, 112

Deville, R., 1, 5, 11, 33, 34, 53, 54, 79, 99, 112

Diestel, J., 65, 67-69, 99, 100, 110, 112

Dundford, N., 109, 112

Enflo, P., 91, 112

Fabian, M., vii, 1, 3-5, 7, 8, 11, 12, 18, $19,22,23,28-30,32,33,35,37$,
$38,40,42,44,47,48,56-59,61-$ $63,70,74,75,83,86,87,90,99$, 112,113

family adequate, 4, 12-14, 16, 17

Farmaki, V., 46, 111, 113

Figiel, T., 74, 109, 112, 113

Floret, K., 70, 113

fragmented, 28

Fremlin, D.H., 38, 113

Giles, J. R., 110, 111

Godefroy, G., 1, 5, 7, 11, 12, 33-35, 37, $38,44,53,54,63-65,74,75,79$, $83,86,99,112-114$

González, A., 114

Granero, A. S., 100, 111

Grothendieck, A., 56, 108, 114

Gul'ko, S. P., 114

Hájek, P., vii, 1, 3, 5, 22, 32, 35, 38, 40, $42,56-59,61,63,70,74,75,83$, $84,86,90,94,96,99,112-114$

Habala, P., 1, 3, 22, 32, 35, 40, 63, 70, 74, $75,83,90,99,113$

Hajnal, A., 114

Jayne, J. E., 114

Jiménez, M., 100, 111

John, K., 85, 114

Johnson, W. B., 109, 112, 114, 115

Köthe, G., 115

Kadec-Klee property, 7, 63, 83

Kadec-Klee* property, 63, 64, 69-72

Kalenda, O., vii 
Kalton, N., 7, 63-65, 75, 114

Kamke, E., 115

Lancien, G., 1, 7, 63-65, 75, 114, 115

Leiderman, A. G., 4, 11, 16, 17, 110, 115

Lindenstrauss, J., 6, 7, 32, 44, 109, 111, 114,115

Markushevich, A. I., 83, 115

Mercourakis, S., 6, 42, 46, 111

Milman, V. D., 64, 115

modulus

of convexity, 73-75, 79, 80

of smoothness, $7,73-75,79,80$

Montesinos, V., vii, 1, 3, 5, 7, 8, 12, 22, $32,35,37,38,40,42,44,56-59$, 61-63, 70, 74, 75, 83, 84, 87, 90, 94, 96, 99, 112-114

Moreno, J. P., 100, 111

Nördlander, G., 115

Namioka, I., 18, 33, 36, 111, 115

norm

C-LKK* ${ }^{*}, 63,64$

C-LKK* ${ }^{*}$ 63-65, 68, 69, 78

DS, 66

LUR, 6, 68, 90, 110

rotund, 49,50

Oncina, L., 110, 115

Orihuela, J., vii, 23, 33, 36, 37, 111, 114, 116

Pallarés, A., 114

Pełczyński, A., 94, 95, 109, 111, 112

Pelant, J., 1, 3, 22, 32, 35, 40, 63, 70, 74, $75,83,90,99,113$

Phelps, R. R., 115

Pisier, G., 75, 116

projectional generator, 2, 4, 21-23, 33, 35 full, 24, 26-28, 30, 32-37

projectional resolution of identity, 5, 23, $24,28,34,87$

separable, 30, 31, 44, 46

Pták, V., 116
Rainwater, J., 6, 110, 116

Raja, M., 110, 115

Rogers, C. A., 114

Rosenthal, H. P., 7, 8, 46, 85, 86, 99, 100, 116

Rudin, M. E., 111

Rychtáŕ, J., 1, 5, 39, 116

Schlüchtermann, G., 109, 116

Schwartz, J. T., 109, 112

selector, 28

set

$\varepsilon$-Asplund, 87

$\varepsilon$-weakly compact, $7,56,57$

$\varepsilon$-weakly self-compact, 7,58

Asplund, 8, 80, 87, 92

asymptotically $\infty$-flat, 85

asymptotically p-flat, 7, 65-69, 71, $72,74-82,85,86,90-92,110$

asymptotically uniformly flat, 64

innerly asymptotically $\infty$-flat, 85

innerly asymptotically p-flat, $7,8,69$, 80-82, 85, 86, 90

Sokolov, G. A., 16, 115

space

Asplund, 5, 7, 28-30, 33, 39, 40, 65, $69,70,83,85-87,90,100,112$ 115

Asplund generated, 87

$c_{0}(\Gamma), 6-8,49-51,57,58,60,63-66$, $68,69,74,75,77,78,82-86,91$, 98-101, 111, 113, 114, 116

DENS, 39

$\mathcal{K}$-countably determined, 4,18

$\ell_{p}(\Gamma), 7,8,65,66,74,78,79,82-86$, 91,113

paired, 4, 11-13, 18

WCD, 2, 4, 5, 11, 21, 24, 33-35, 37, 40

WCG, 2, 4, 6, 7, 21, 23, 24, 31, 32, $40,42,45-47,56,64,65,75,85$, $86,90,111,113,116$

WLD, 2, 4, 5, 21, 24, 35, 37-40, 85, 86 
Tacon, D. G., 116

Talagrand, M., 116

Terenzi, P., 38, 116

topological space

angelic, 5, 37-39, 59, 60, 70, 91, 92, 108

Tzafriri, L., 115

Uhl, J., 99, 100, 112

Valdivia, M., vii, 5, 23, 37-40, 116, 117

Vanderwerff, J. D., 1, 5, 22, 36, 38, 40, $42,84,94,96,110,111,114,117$

Vera, G., 114

Wage, M., 111

Wheeler, R. F., 18, 109, 115, 116

Whitfield, H. M., 36

Whitfield, J. H. M., 117

Wojtaszczyk, P., 8, 101, 107, 117

Xu, H. K., 79, 117

Zippin, M., 117

Zizler, V., vii, 1, 3, 5, 7, 8, 11, 12, 22, $32-38,40,42,44,53,54,56-59$, 61-63, 70, 74, 75, 79, 83-87, 90, $94,96,99,112-114,117$ 